\title{
Prodromus Sinsistemático de la República Argentina Y UNA BreVe INTROdUCCIÓN A los Estudios Fitosociológicos
}

\author{
EDUARDO MARTÍNEZ CARRETERO*, \\ ANA MARÍA FAGGI ${ }^{1}$, JOSÉ L. FONTANA², PABLO ACEÑOLAZA ${ }^{3}$, \\ RICARDO GANDULLO ${ }^{4}$, MARCELO CABIDO5 \\ DAVID IRIART ${ }^{2}$, DARIÉN PRADO ${ }^{6}$, \\ FIDEL ANTONIO ROIG ${ }^{\dagger}$ y ULRICH ESKUCHE ${ }^{\dagger}$
}

\begin{abstract}
Resumen: Se presenta el Prodromus de la vegetación de Argentina, que constituye la primera versión del sinsistema nacional detallado hasta nivel de asociación y subasociación. Se describen las 57 clases de vegetación hasta ahora registradas para el país y la presencia posible de otras 16 . Se indican además las comunidades vegetales sin rango taxonómico. Todas las unidades de vegetación se enmarcan en el esquema fitogeográfico vigente.
\end{abstract}

Palabras clave: Prodromus sinsistemático, Argentina, sintaxones, fitosociología.

Summary: Sinystematic Prodromus of Argentina and a brief introduction to the Phytosociological Studies. The Argentine vegetation Prodrome is presented; it constitutes the first version of the national synsystem detailed to association and sub-association level. In addition, the 57 vegetation classes until know for the country are described, and the possible presence of other 16 . Plant communities without a syntaxonomic level are included. All vegetation units are framed into the current phytogeographic scheme.

Key words: Synsystematic Prodrome, Argentina, sintaxa, phytosociology.

\section{Prólogo}

Hasta el momento prácticamente se carece de ordenamientos sintaxonómicos de la vegetación en los países sudamericanos. Ello se debe a que por lo general sólo existen trabajos fitosociológicos aislados, si bien algunos muy importantes, dejando extensas regiones sin resolver desde este punto de vista. Sin embargo, es factible la confección de catálogos de lo que hasta ahora se ha estudiado. Basados en esta premisa hemos elaborado esta primera contribución para Argentina intentando sintetizar la dispersa, pero abundante, información disponible.

"Geobotánica y Fitogeografía (CCT Mendoza -exCRICYTCONICET)

1 Universidad de Flores (UFLO) Buenos Aires-CONICETAAFi

${ }^{2}$ Universidad Nacional del Nordeste, Corrientes-AAFi

${ }^{3}$ CICYTTP-CONICET-AAFi

${ }^{4}$ Universidad Nacional de Cinco Saltos, Río Negro

${ }^{5}$ IMBIV-Universidad Nacional de Córdoba-CONICET-AAFi

${ }^{6}$ IICAR-Universidad Nacional de Rosario-CONICET
Por otra parte, brindamos una visión sintética de la Fitosociología y sus metodologías derivadas actuales como la Sinfitosociología y la Fitosociología dinámico catenal o Paisajista, que hacen a la estructura teórica de estos estudios florísticos y que otorgan mayor certeza a la definición de las unidades fitogeográficas.

El término Prodromus proviene de la medicina donde significa primeros síntomas, evidencias, de una enfermedad. En Fitosociología se lo propuso para todo trabajo preliminar tendiente a elaborar un detallado sinsistema (Géhu, 2006). El proyecto de Prodromo comenzó en 1933 en Europa auspiciado por el Comité Internacional de Prodromus Fitosociológico, coordinado por J. Braun-Blanquet; posteriormente, en 1952, Braun-Blanquet, Roussine y Nègre publicaron el Prodromus de las comunidades vegetales de la Francia mediterránea; en 1973, Tüxen inició el Prodromus der europäischen Pflanzengesellschaften (Prodromo europeo de comunidades vegetales) coordinado por la Asociación Internacional de Fitosociología, y en 2004 se publicó el Prodrome des végétations de France (Bardat et al., 2004). 
El Prodromus ofrece una clasificación de referencia de la vegetación de Argentina, útil para los fitosociólogos en los trabajos de campo, para los administradores de áreas protegidas y para establecer políticas sobre la conservación, manejo y restauración de los recursos vegetales.

\section{Criterios seguidos en la elaboración del Prodromus}

Se mencionan los sintaxones que se han publicado para Argentina incluyendo aquellos de países vecinos cuya presencia es citada para nuestro territorio.

Cada clase va acompañada de una breve descripción de sus características ecológicas. Cada sintaxón lleva el autor o autores y el año de su publicación que remite a la correspondiente bibliografía. En la mayoría de los sintaxones, a nivel de asociación, se incluye el relevamiento tipo.

En casos de sintaxones publicados en los que no se haya cumplido con las exigencias del tipo nomenclatural, y donde ha sido posible, se ha elegido un lectotipo (Código, Definición VIII, art. 19).

En los sintaxones con tres o más autores, la totalidad de los mismos se agrega en el primero que se cita, en los siguientes sintaxones se indica: ídem auctores.

Las asociaciones no ubicadas sintaxonómicamente y otros sintaxones sin rango (comunidad, nodum, Gesellschaft, Soziation, etc.) (Código, Definición I) se han agrupado según territorios florísticos y provincias fitogeográficas. Existe en el país una abundante bibliografía de este tipo que constituye una valiosa información de base que consideramos indispensable dar a conocer.

Abreviaturas utilizadas
corr. correxit, corregido
ex según
em. emendavit, enmendado
et al. et alterum, y otros
idem auctores los mismos autores
inéd. inédito
nom. inval. nomen invalidatus, nombre invalidado
nom. mut. nomen mutantis, n.m., nombre que cambia
nom. mut. propos. nomen mutantis propositium, cambio
de nombre propuesto
nom. nov. nomen novus, nombre nuevo
prov. provisional
val. validado
Rel. relevamiento florístico
TBPA Transecta Botánica de la Patagonia Austral
(?) sintaxón que puede estar representado en la Argentina
(T) Tipo nomenclatural para el sintáxon próximo superior

\section{INTRODUCCIÓN}

Grisebach en 1879 publicó el Symbolae ad Floram Argentinam sobre la flora del centro y noreste de nuestro país; previamente, en 1872 publicó Die Vegetation der Erde donde trata el Dominio de las Pampas, siendo el primer aporte fitogeográfico argentino. Inmediatamente la Academia Nacional de Ciencias publicó Observaciones sobre la vegetación de Tucumán, de Hieronymus, que constituyó el primer ensayo de carácter sintaxonómico. Argentina era el país sudamericano a la vanguardia de los estudios florísticos-fitogeográficos. Entre fines del siglo XIX e inicios del siglo $\mathrm{XX}$ se produjo una intensa tarea fitogeográfica y de cartografía de la vegetación, por lo que Parodi (1961) consideró al año 1905 como el de inicio de la fase contemporánea en la historia botánica (geobotánica) argentina. Podemos considerar una segunda fase hasta 1930 cuando Parodi publica su Ensayo Fitogeográfico del Partido de Pergamino, $\mathrm{y}$ desde entonces y hasta la actualidad la tercera fase con intensa participación de botánicos argentinos. En Argentina existe una rica información histórica sobre los estudios de la vegetación que debe tenerse en cuenta en cuanto fundamenta los conocimientos actuales, entre otras contribuciones se deben mencionar: Alboff (1896), Alfonso (1940), Ambrosetti, Méndez \& Roig (1968), Anderson, Del Aguila \& Bernardon (1970), Auer (1933a,b, 1939, 1941, 1963), Azara (1809), Báez (1922, 1938, 1939, 1942, 1956, 1969), Bailey Willis (1914), Birger (1906), Böcher (1958), Boelcke (1957), Bonvisutto (1989), Brackebusch (1893), Bragadin (1959), Burkart (1957, 1969), Burmeister (1861), Cabrera (1934, 1936, 1940a,b, 1941, 1943a,b,c, 1944, 1945a,b, 1948, 1949, 1955, 1958, 1968, 1969, 1971), Cabrera \& Dawson (1944), Cabrera \& Abbiatti (1945), Cabrera \& Willink (1973), Cano \& Movia (1967), Cano \& Gómez Cadret (1968), Carette \& Ruiz Leal (1939), Castellanos (1927, 1938, 1958), Correa Luna ( 1955, 1964), Czajka \& Vervoorst (1956), Devito (1951), Dimitri (1959, 1960, 1962, 1972a,b,c), Dimitri et al. (1974), Donat (1931, 1932, 1934, 1935, 1936, 1937), Dusén (1898, 1905), Faggi (1995), Frangi (1976), Fries (1905), Fiebrig \& Rojas (1933), Gallopin (1978), Gómez et al. (1997), Goodall (1972), Grondona (1951), Haumman (1913a,b, 1918, 1919, 1920a,b, 1925, 1926), Heim (1941), Hemsley (1886), Herzog (1931), Hieronymus (1874), Holmberg (1882), Hosseus (1915, 1916a,b, 


\section{E. Martínez Carretero et al. - Prodromus Sinsistemático y Estudios Fitosociológicos}

1922), Hueck (1950, 1951a,b, 1952, 1953, 1954, 1957, 1959, 1960-61, 1966), Hunziker (1952), Ibañez (1962), Ihering (1929), Kalela (1946), Kanter (1936), Knapp (1964, 1965), Koutché (1928), Kühn (1930), Kühn \& Rohmeder (1943), Kühnemann (1970, 1972, 1976), Kurtz (1886), León \& Suero (1962), León et al. (1998), Lewis \& Collantes (1974, 1975), Lillo (1919), Ljungner (1939), Lorentz (1876, 1878), Mackenzie Lamb (1958), Manzi (1961), Martín (1949, 1955), Martínez Crovetto (1950, 1963, 1967), Monticelli (1938), Morello (1952, 1955, 1958, 1967, 1970), Morello \& Adámoli (1968, 1974), Morello et al. Saraceno (1971), Meyer (1936, 1940, 1943, 1944, 1959, 1963), Neger ( (1896, 1897), Niederlein (1881, 1883), Offner (1907), Parodi (1929, 1930, 1934, 1940a,b, 1941), Perez-Moreau (1935, 1944, 1945), Poi de Neiff \& Neiff (1977), Ragonese (1936, 1941, 1951, 1967), Ragonese \& Castiglioni (1970), Ragonese \& Covas (1940, 1947), Ragonese \& Piccinini (1969, 1977), Ringuelet (1938, 1939, 1947), Rodríguez García et al. (1978), Rohmeder (1942a,b, 1943, 1945, 1947, 1955), Roig (1955a,b, 1971, 1972), Roig \& Roig (1969), Roig \& Ruiz Leal (1959), Roivainen (1954), Roquero (1968), Rühle (1928), Ruiz Leal (1951, 1954, 1972), Ruiz Leal \& Roig (1958, 1959, 1964, 1965), Ruthsatz (1978), Ruthsatz \& Movia (1975), Sayago (1969), Schenck (1905), Schmieder (1923, 1928, 1929), Schreiter (1936), Schulz (1961), Seckt (1912, 1943), Seibert (1972), Singer (1971), Skottsberg (1904, 1905, 1906, 1907, 1909a,b, 1910, 1913, 1916, 1921a,b,c, 1931, 1942, 1945, 1960), Soriano (1949, 1950, 1956), Tell \& Bonetto (1978), Tur (1972), Tweedie (1840), Varela et al. (1978), Verettoni (1961, 1965), Vervoorst (1951a,b), Walter (1967a,b), Ward \& Dimitri (1966), Werner (1972, 1974), Werth (1906-1908), Will (1887, 1890), Zaixso \& Pastor (1977). Sin embargo, los estudios florísticos no avanzaron al ritmo necesario para sentar una geobotánica y un conocimiento adecuado de la florística argentina (Hauman, 1931; Roig, 1990).

El avance actual de la frontera agropecuaria, la erosión hídrica y eólica, la pérdida de especies, la modificación en la estructura y composición de los sistemas naturales, el avance urbano, etc. requieren de un mayor conocimiento de la estructura y funcionamiento de los diversos ecosistemas y es aquí donde los estudios florísticos-geobotánicos realizan aportes imprescindibles.

\section{La Geobotánica}

Los estudios de la vegetación, distribución de las especies, zonación en función de diferentes parámetros ambientales, etc., dieron un paso significativo a partir de A. von Humboldt (1845) con su obra Kosmos donde sentó las bases para el estudio científico del paisaje vegetal e inició la Geobotánica, proponiendo los estudios de las plantas y sus relaciones con los climas regionales y generales. Desde entonces, la relación clima, topografía, suelo, vegetación, comenzó a considerarse como una entidad a ser estudiada. En ese contexto la Geobotánica o Geografía botánica o Ecología vegetal o Geobotánica Global o Integradora estudia la diversidad de los complejos vegetales y su relación con el ambiente (hábitat), echando mano a las diversas teorías del estudio de la vegetación. Además, vincula los conocimientos empíricos del uso de los recursos naturales (uso tradicional), especialmente los de génesis antigua o no renovables, para lograr su uso más eficiente y una conservación más eficaz.

La Geobotánica se nutre de otras disciplinas científicas como la bioclimatología, geología, geomorfología, edafología, ecofisiología, corología, taxonomía, fitosociología, que aportan a definir su marco conceptual.

Es necesario avanzar en el estudio de la vegetación superando controversias en cuanto al modo de organización de las plantas (en unidades continuas o discretas). Cuando el análisis de la vegetación parte de un inventario minucioso, alcanzando el nivel específico o infra-específico, en un área concreta y fisiográficamente homogénea a la escala de trabajo, se pueden establecer unidades ambiente-vegetación, o comunidades vegetales, que contienen importante información mesológica y florística. El concepto comunidad vegetal adquiere así dimensión real y permite configurar un modelo de análisis y un modelo sintaxonómico (Anand \& Orlóci, 1997; Mucina \& van der Maarel, 1989; Moravec, 1989, 1992; Feoli, 1998; Yu \& Orlóci, 1990; Loidi, 2004; Biondi et al., 2004, 2011).

Dentro de la Geobotánica podemos ubicar a la Fitosociología, rama del conocimiento que estudia las comunidades vegetales y su relación con el medio, además de los procesos que las modifican (fitocenosis). Sociología Vegetal y Sinecología Vegetal son términos sinónimos comúnmente empleados. El análisis de los relevamientos permite definir las comunidades vegetales. Cuando las comunidades se 
ordenan jerárquicamente, en un sistema tipológico sintaxonómico, la asociación es la unidad fundamental. Desde la propuesta inicial de Braun-Blanquet (1921) (hoy llamada Fitosociología clásica) se ha avanzado a la Fitosociología Dinámico-Catenal y Permacatenal o Fitosociología Paisajista basada en el sigmetum (o serie) (unidad geotopo-vegetación).

En este contexto, la asociación es una comunidad vegetal concreta con cualidades mesológicas determinadas, encuadre geográfico definido y combinación propia de especies (o especies características), sucesionalmente correspondiente a una etapa estructuralmente estable. A la definición de la asociación se llega mediante el estudio comparado de los relevamientos. En el ordenamiento tipológico, las asociaciones de composición florística y hábitat similar en un encuadre biogeográfico semejante se reúnen en categorías de orden superior: Alianzas, Órdenes y Clases (Braun-Blanquet, 1921, 1928, 1948, 1964; Braun-Blanquet et al., 1947; Géhu \& RivasMartínez, 1981, 1985, 1987; Pignatti, 1990; Loidi, 1994, 1999, 2004; Dierschke, 1994; Izco, 1998).

La Sinfitosociología y la Geosinfitosociología son disciplinas que derivan de la Fitosociología en el intento de alcanzar una visión paisajísta de la vegetación. La Sinfitosociología parte de una concepción previa del paisaje con un criterio geográfico, mientras que la Geosinfitosociología emplea un criterio geomorfológico. En ambas el sigmetum es la unidad básica y requiere el análisis dinámico. La tarea en terreno se resuelve a través del sinrelevamiento o inventario de los agrupamientos vegetales; en el sigmarelevamiento se parte de las comunidades vegetales que ocurren en el paisaje, mientras que en el geosigmarelevamiento desde los elementos del relieve.

La Fitotopografia, Landscape o Landschaft consiste en el estudio y descripción del paisaje vegetal a través de sus comunidades vegetales, enfoque basado en ideas previas como la Assoziationskomplex (Du Rietz, 1921) y en el Complex de groupements (Braun Blanquet \& Pavillard, 1928).

La Fitosociología Dinámico-Catenal y Permacatenal trata el estudio del Paisaje Vegetal. La unidad de análisis la constituye la serie de vegetación o sigmetum. Esto es, el conjunto de comunidades vegetales ubicadas en espacios (geomorfologías) similares resultados de la sucesión; es decir, incluyen las características mesológicas, geográficas y florísticas de la asociación de la etapa estable y de las asociaciones que las puedan reemplazar. En las categorías tipológicas las unidades son, básicas: sigmetum (serie), permasigmetum (permaserie), geosigmetum (geoserie) y geopermasigmetum (geopermaserie), con sus respectivas subunidades; de orden superior: sigmion, sigmetalia, sigmetea; geosigmion, geosigmetalia, geosigmetea, permasigmion, persigmetalia, permasigmetea; geopermasigmion, geopermasigmetalia y geopermasigmetea (Tabla 1).

Las series, a su vez, pueden ser climatófilas, ubicadas en suelos maduros y dependientes sólo del agua de lluvia; edafoxerófilas, en suelos xerofíticos y edafohigrófilas, en suelos húmedos. En la Tabla 1 se indica de manera comparativa la relación entre las unidades (Rivas-Martínez, 1994, 2005).

El sigmetum (serie de vegetación) representa el conjunto de comunidades vegetales (y sus estadios o etapas) en espacios teselares afines

Tabla 1. Unidades de vegetación en la Fitosociología dinámico-catenal.

\begin{tabular}{|lll|}
\hline \multicolumn{1}{|c|}{ Unidades } & \multicolumn{1}{c|}{ Unidad tipo } & \multicolumn{1}{c|}{ Unidades principales superiores } \\
\hline Sintaxones & Asociación & Alianza, orden, clase \\
Sigmataxones & $\begin{array}{l}\text { Sigmetum } \\
\text { Serie de vegetación }\end{array}$ & $\begin{array}{l}\text { Sigmion, sigmetalia, sigmetea } \\
\text { Macroserie, megaserie, hiperserie }\end{array}$ \\
Permasigmataxones & $\begin{array}{l}\text { Permasigmetum } \\
\text { Permaserie de vegetación }\end{array}$ & $\begin{array}{l}\text { Permasigmion, permasigmetalia, permasigmetea } \\
\text { Geosigmataxones }\end{array}$ \\
& $\begin{array}{l}\text { Geosigmetum } \\
\text { Geoserie de vegetación }\end{array}$ & $\begin{array}{l}\text { Geosigmion, geosigmetalia, geosigmetea } \\
\text { Macrogeoserie, megageoserie, hipergeoserie }\end{array}$ \\
& & $\begin{array}{l}\text { Geopermasigmion, geopermasigmetalia, } \\
\text { Geopermasigmataxones }\end{array}$ \\
& $\begin{array}{l}\text { Geopermasigmetum } \\
\text { Geopermaserie de vegetación }\end{array}$ & $\begin{array}{l}\text { Macrogeopermaserie, megageopermaserie, } \\
\text { hipergeopermaserie }\end{array}$ \\
\hline
\end{tabular}




\section{E. Martínez Carretero et al. - Prodromus Sinsistemático y Estudios Fitosociológicos}

producto de la sucesión; es decir, incluye las características del ambiente, geográficas y florísticas de la asociación que representa la etapa estable (climácica) y aquellas asociaciones que las pueden reemplazar. En la Fitosociología Dinámica, el sigmetum es la unidad básica. Se pueden distinguir tres series, la climatófila, corresponde a las comunidades que se ubican en suelos maduros según el mesoclima (dependen sólo de las lluvias), las edafoxeres, en suelos xerofíticos (psammófilas, gipsófilas, etc.) y las edafohigrófilas, en suelos húmedos (histosoles, salares, palustres, etc.) (Tabla 2).

Para denominar, y diagnosticar, cualquier serie de vegetación junto con el nombre común se agrega una frase descriptiva breve de las características de hábitat

Tabla 2. Series de vegetación asociadas al sigmetum.

\begin{tabular}{|cl|}
\hline Sigmetum & Serie climatófila (Sigmetum acidófilo, S. neutrófilo, S. basófilo) \\
(Series de vegetación) & Serie edafoxerófila (Sigmetum psamófilos, S. de litosuelos, etc.) \\
& Serie edafohigrófila (Sigmetum fluvial, S. halófilo, S. palustre, etc.) \\
\hline
\end{tabular}

y biogeografía (piso bioclimático, suelo, ubicación fitogeográfica, etc.) y la especie dominante (Tabla 3). Las unidades de rango inferior (subseries) como las de rango superior (macro, mega o hiperseries) corresponden a subasociaciones o a subalianzas, alianzas, órdenes y clases, respectivamente. En la denominación latina de cada unidad se emplea, según el rango: sigmetosum, sigmetum, sigmion, sigmetalia, sigmetea.

En la Fitosociología Dinámico-Catenal o Paisajista (geosigmataxones), la geoserie o geosigmetum es la unidad básica. En este caso

\section{Tabla 3. Series de vegetación.}

\begin{tabular}{|c|c|c|}
\hline Unidades & Unidad básica y de rango superior & Referencias nomenclaturales \\
\hline $\begin{array}{l}\text { Sigmataxones } \\
\text { o } \\
\text { Sigmetos }\end{array}$ & $\begin{array}{l}\text { Serie de vegetación: } \\
\text { macroserie, megaserie, hiperserie } \\
\text { Sigmetum: sigmion, sigmetalia, sigmetea }\end{array}$ & $\begin{array}{l}\text { Asociación representativa de la } \\
\text { etapa estable (cabeza de serie) } \\
\text { de la serie de vegetación y de los } \\
\text { sintaxones que la engloban. }\end{array}$ \\
\hline $\begin{array}{c}\text { Geosigmataxones } \\
\text { o } \\
\text { Geosigmetos }\end{array}$ & $\begin{array}{l}\text { Geoserie de vegetación: } \\
\text { macrogeoserie, megageoserie, } \\
\text { hipergeoserie } \\
\text { Geosigmetum: geosigmion, } \\
\text { geosigmetalia, geosigmetea }\end{array}$ & $\begin{array}{l}\text { Asociación representativa de la } \\
\text { etapa estable (cabeza de serie) de la } \\
\text { serie de vegetación preponderante } \\
\text { en las catenas fluviales, y de los } \\
\text { sintaxones que la engloban. }\end{array}$ \\
\hline $\begin{array}{c}\text { Geopermasigmataxones } \\
\text { o } \\
\text { Geopermasigmetos }\end{array}$ & $\begin{array}{l}\text { Geopermaserie de vegetación: } \\
\text { macrogeopermaserie, megageopermaserie, } \\
\text { hipergeopermaserie } \\
\text { Geopermasigmetum: geopermasigmion, } \\
\text { geopermasigmetalia, geopermasigmetea }\end{array}$ & $\begin{array}{l}\text { Asociación quionófoba altioreina y litoral } \\
\text { preponderante de las permaseries de } \\
\text { vegetación de las catenas concernidas, } \\
\text { y de los sintaxones que la engloban. }\end{array}$ \\
\hline
\end{tabular}

corresponde a una catena de sigmetum (o de series de vegetación) ubicados en un piso bioclimático y territorio biogeográfico determinado y que pueden alternarse según las condiciones del suelo. Prácticamente se integran por un conjunto de sigmetum contiguos dentro de una unidad geomorfológica determinada (llanura, turbera, río, valle, piedemonte); sin embargo, el marco general de cualquier catena completa incluye las unidades geomorfológicas de cresta-ladera-valle, que sintetizan las condiciones xéricas-mésicashigrófilas. En este marco general se ubican las 
comunidades vegetales y es donde ocurren las etapas sucesionales y catenales.

Se pueden distinguir dos geosigmetum o geoseries de vegetación: el topográfico y el cliserial. El topográfico o geomorfológico refiere al modelo general de cresta-ladera-valle (que cuando se encuentra completo agrupa las series edafoxerófilas, climatófilas y edafohigrófilas). El geosigmetum cliserial agrupa las series climatófilas (por ejemplo de contigüidad altitudinal en áreas montañosas, lo que comprendería al menos dos pisos de vegetación termoclimáticos). Al nombrar (y diagnosticar) una geoserie de vegetación al nombre común se le agrega una frase breve descriptiva de las condiciones del ambiente, biogeografía, topografía, suelo, etc. y la especie dominante; las unidades de rango superior son: geosigmion, geosigmetalia y geosigmetea.

Los Permasigmetum o permaserie de vegetación, son comunidades vegetales estables ubicadas en teselas o complejos teselares afines en territorios como las altas cumbres, acantilados, médanos activos, turberas, márgenes de lagunas, etc., en las que la etapa estable (o madura) es una comunidad vegetal de vasculares, poco estratificada, sin comunidades serales (excepto las anuales que temporalmente se hacen presente); de esta manera, las plantas vivaces reorganizan la comunidad vegetal permanente. Las unidades de rango superior se denominan: permasigmetum, permasigmion, permasigmetalia, permasigmetea.

La expresión catenal de un conjunto de permasigmetum vecinos, delimitados topográfica o edáficamente, es la Geopermaserie o geopermasigmetum. En este caso la microtopografía determina condiciones microclimáticas o edáficas generando diversidad de hábitats con comunidades vegetales permanentes, vivaces. Para el estudio de estas comunidades vegetales hay que situarse en un piso bioclimático determinado y en un enclave geomorfológico preciso y siguiendo el factor ecológico determinante de la catena. Al nombrar (y diagnosticar) una geopermaserie de vegetación al nombre común se le agrega una frase breve con las condiciones del hábitat, piso bioclimatico, topografía, etc.

En la serie, permaserie, geoserie o geopermaserie de vegetación se encuentran unidades de rango inferior denominadas Faciaciones de vegetación.
Éstas permiten distinguir comunidades o conjunto de comunidades vegetales potenciales diferentes del tipo descriptivo del sigmetum, permasigmetum, geosigmetum o geopermasigmetum. Es decir, es vegetación ligada entre sí sucesionalmente o por el medio pero no suficientemente diferentes para constituir series. Las faciaciones (geofaciaciones) sirven para expresar variaciones vegetacionales por la intensa utilización del territorio por el hombre (uso agrícola, ganadero, forestal, etc.).

\section{La Fitosociología}

Es necesario presentar una síntesis del método fitosociológico, base de los modernos enfoques en los estudios florísticos-fitogeográficos de una región.

Un paso trascendente en la ciencia de la vegetación fue la introducción del concepto de formación vegetal por Grisebach en 1838, con sentido fisionómico. Posteriormente, este concepto se enriqueció con los criterios sucesionistas angloamericanos, hasta llegar a las aproximaciones fitogeográficas como las de Ellenberg y Mueller-Dombois (1967) y MuellerDombois y Ellenberg (1974) propiciadas por la Unesco (Díaz González, 2004).

A inicios del Siglo XX dos escuelas anglosajonas y una europea debatían sobre la vegetación. La organísmica y co-evolutiva de la vegetación de Clements (1904) y la individualista de la vegetación de Gleason (1917), por un lado, y la de BraunBlanquet $(1921,1958,1964)$ y Tüxen $(1956,1979)$ por el otro (Fig. 1).

En la visión Clementsiana y europea las comunidades vegetales son naturales y se componen de conjuntos de especies que reiteradamente ocurren juntas; mientras que en la anglosajona, los gradientes son tan diversos y numerosos por la ocurrencia simultánea de diversos factores (bióticos, ambientales) que cada combinación de especies resultaría irrepetible. La visión más realista, integrada, de Gauch y Whittaker, conocida como de patrón climáxico, considera que la vegetación de una región particular se distribuye como un mosaico, incluyendo los ecotonos. Para Goodall (1952), las especies aumentan indefinidamente con el logaritmo del área sin llegar a una asíntota (Fig. 2). Cuando varias especies están asociadas en una comunidad se asume que tendrán curvas de abundancia similares en relación a un factor ambiental. Sin embargo, en 


\section{E. Martínez Carretero et al. - Prodromus Sinsistemático y Estudios Fitosociológicos}
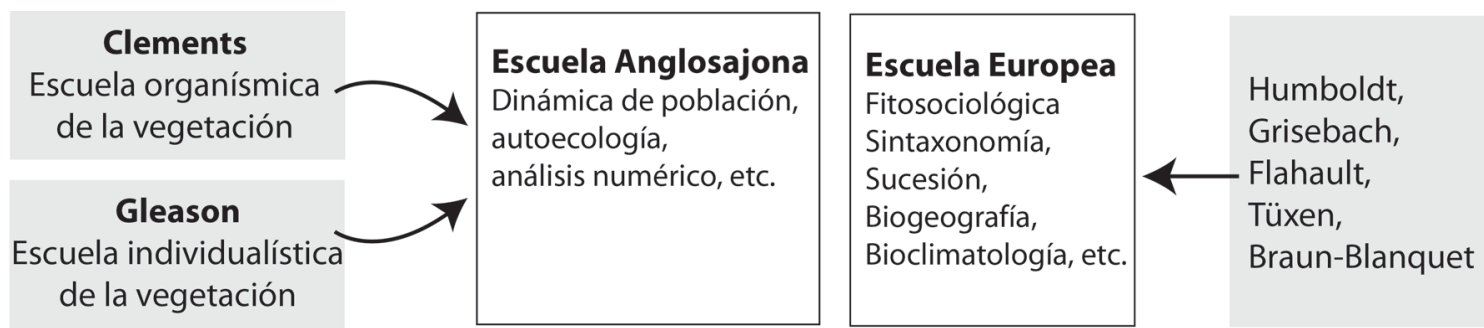

Fig. 1. Escuelas anglosajonas y europea en el estudio de la vegetación.

casos concretos las curvas varían en ancho y alto indicando diferentes rangos de tolerancia.

Si bien en la visión integradora actual estas posturas están perimidas, marcaron durante varias décadas el devenir de los estudios de la vegetación. El enfoque moderno, holístico, de la fitosociología integradora se acerca significativamente al enfoque de la Teoría General de Sistemas (Loidi, 2001).

El primer ensayo organizado de cartografía de la vegetación a nivel mundial se debe a Flahault, en 1894, mediante el proyecto "Carta Botánica, Agrícola y Forestal de Francia" y la publicación de la Carta de Perpignan a escala 1:200000 (Flahault, 1897). Posteriormente, en 1915, Braun-Blanquet en su tesis sobre la vegetación de L'Aigual (Cevennes meridionales, Francia) pone en juego el concepto de asociación vegetal. Braun-Blanquet y Pavillard, en 1928, publican el Vocabulaire de Sociologie Vegetale donde aparece por primera vez el término Sociología. A partir del Congreso Internacional de Amsterdam, en 1935, el concepto de asociación se emplea internacionalmente y permitió el desarrollo posterior de otros como: el dinámico (Sinfitosociología), el corológico (Biogeografía), el climático (Bioclimatología), etc.

Actualmente, se reconocen tres niveles de aproximación al análisis del paisaje vegetal de un territorio: el fitosociológico (las comunidades
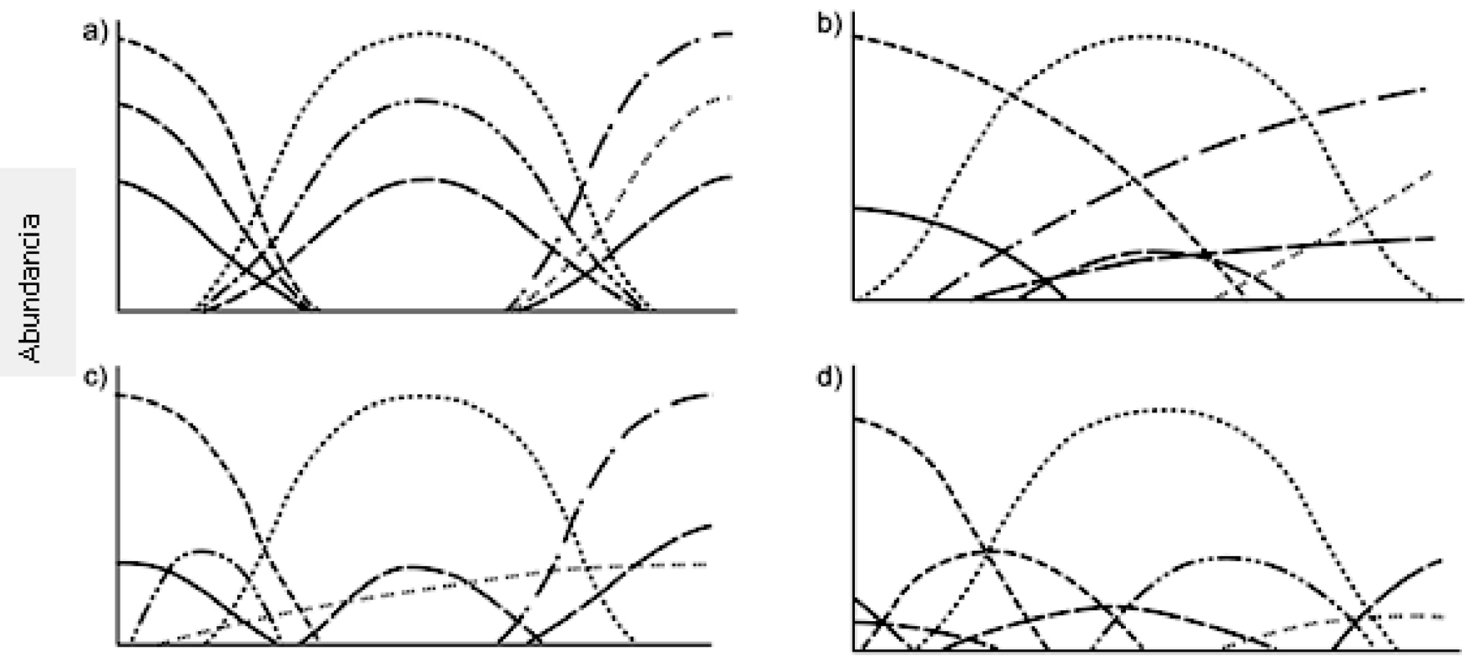

Gradiente ambiental

Fig.2. Abundancia de especies a lo largo de gradientes ambientales. (a) visión Clementsiana $(1916,1928)-$ europea (comunidad); (b) visión individualista (Continuum) según Gleason (1939); (c) según la hipótesis del patrón climáxico de Gauch \& Whittaker (1972); (d) respuestas de las curvas de las especies según Goodall (1952) (adaptada de Mota et al., 2004). 
Tabla 4. Comparación entre diferentes modos de estudio de la vegetación.

\begin{tabular}{|c|c|c|c|c|}
\hline $\begin{array}{l}\text { Nivel de } \\
\text { Organización }\end{array}$ & Florístico & Vegetación & Paisaje Sucesional & Catenal O Politeselar \\
\hline $\begin{array}{l}\text { Disciplina } \\
\text { cientifica }\end{array}$ & Taxonomía & Fitosociología & Sinfitosociología & Geosinfitosociología \\
\hline Unidad básica & Especie & Asociación & $\begin{array}{l}\text { Serie de vegetación } \\
\text { / Sigmetum }\end{array}$ & $\begin{array}{l}\text { Geoserie de vegetación } \\
\text { / Geosigmetum }\end{array}$ \\
\hline $\begin{array}{l}\text { Unidad de } \\
\text { muestreo }\end{array}$ & Individuo & Individuo de asociación & Tesela & Catena \\
\hline $\begin{array}{l}\text { Unidad de } \\
\text { trabajo }\end{array}$ & $\begin{array}{l}\text { Material } \\
\text { herborizado }\end{array}$ & $\begin{array}{l}\text { Inventario } \\
\text { (relevamiento) }\end{array}$ & Sininventario & Geosininventario \\
\hline \multicolumn{5}{|l|}{ Esquema } \\
\hline $\begin{array}{l}\text { Nombre } \\
\text { científico }\end{array}$ & $\begin{array}{l}\text { Larrea } \\
\text { divaricata }\end{array}$ & $\begin{array}{l}\text { Larreetum divaricato- } \\
\text { cuneifoliae }\end{array}$ & Larreetalia & Larreetea \\
\hline
\end{tabular}

vegetales) de base florística y ecológica que permite la definición de las asociaciones, su ordenamiento jerárquico (sintaxa) y su ecología (sinecología), el sinfitosociológico o complejos de comunidades vegetales y sus relaciones dinámicas, por ende se definen las series dinámicas (sigmeta), y el geosinfitosociológico (geoseries de vegetación o geosigmetum) o fitosociología catenal o de relaciones geográficas entre diferentes series de vegetación que lleva a la identificación de unidades fitogeográficas o de paisaje (geosigmeta). Una serie de vegetación expresa el conjunto de comunidades vegetales que se hallan en espacios teselares afines como resultado de la sucesión; incluye por lo tanto las cualidades mesológicas, geográficas y florísticas de la asociación representativa (Díaz González, 2004) (Tabla 4).

Estas aproximaciones le confieren a la fitosociología valor bioindicador de la calidad ambiental a través de las comunidades vegetales, grupo de asociaciones o de las series de vegetación (Biondi, 2011).

Literalmente Fitosociología significa sociología de plantas. Las plantas viven en comunidades compitiendo por el espacio, agua, nutrientes, luz. La Fitosociología busca conocer esas sociedades o comunidades. Braun-Blanquet propuso un método de base florística para estudiar las comunidades vegetales por lo que es común la identificación como método de Braun-Blanquet o sigmatista (SIGMA: Station Internationale de Geobotanique Méditerranée et Alpine) o escuela de ZurichMontpellier. Bajo este enfoque se acepta que las comunidades vegetales son naturales (reales) y se componen de un conjunto de especies que reiteradamente ocurren juntas.

Comúnmente se clasifica y ordena la vegetación según su fisonomía, fenología, respuesta espectral (electromagnética o formas biológicas), respuesta a variables ambientales (geoformas, litología, humedad, etc.), su dinámica temporal o composición específica. La Fitosociología acepta la taxocenosis como punto de partida para el estudio de la comunidad; es decir, se incluye un grupo o unos pocos grupos taxonómicos aunque exista estrecha relación con los demás organismos (Mota et al., 2004). Así como es difícil encontrar dos tipos de vegetación con idéntica composición florística, es posible hallar ciertos grupos de especies presentes en práticamente todas las muestras. La asociación es una unidad abstracta que busca representar una comunidad vegetal de composición florística definida.

La asociación define un sistema de organismos vegetales con una composición florística que es estadísticamente repetitiva, posee una estructura, valor ecológico y calidad de las relaciones dinámicas y catenales propios respecto de otras comunidades. 


\section{E. Martínez Carretero et al. - Prodromus Sinsistemático y Estudios Fitosociológicos}

Posee un complejo especifico característico; es decir, plantas preferentes potencialmente ligadas en términos estadísticos y que son biogeográfica y ecológicamente diferenciales comparadas con asociaciones sinvicariantes o geosinvicariantes. El complejo específico característico permite reconocer a un sintaxón a través de las especies extraídas por medio de análisis numéricos (Biondi, 2011).

El estudio se estructura en tres etapas: analítica, sintética y de clasificación o sintaxonómica. En la analítica se toman los datos a campo (relevamientos o inventarios), en la sintética se analizan mediante tablas o matrices y en la sintaxonómica las asociaciones se clasifican jerárquicamente.

Para comprender la fitosociología se deben tener en cuenta las premisas:

-una comunidad vegetal es un tipo de vegetación reconocido a través de su composición florística; mientras más completa mejor expresa las relaciones interespecíficas y con el ambiente.

-algunas especies de la comunidad indican mejor que otras esas interrelaciones y se las denomina especies diagnóstico (características y diferenciales).

-las especies diagnóstico permiten organizar las comunidades vegetales en un sistema jerárquico donde la asociación es la unidad elemental o básica.

Al ser la composición florística la base del método, la determinación de cada especie integrante de la comunidad debe ser precisa, así como la evaluación de los parámetros ambientales considerados relevantes.

Debe tenerse en cuenta que cada especie posee un conjunto de exigencias respecto del medio donde vive, por lo que las especies que ocupan un determinado ambiente presentan exigencias semejantes, siendo ésta la base de la Fitosociología. Como las exigencias o intensidad de las exigencias no son siempre las mismas para todas las especies se aplica el concepto de amplitud ecológica, que es la mayor o menor capacidad de una especie para poblar un área determinada. Así, especies de gran amplitud ecológica o especies "euri" pueden ocupar diversos ambientes, mientras que las especies "steno", de poca amplitud ecológica y de mayor exigencia del ambiente, son indicadores más eficaces del medio que habitan.

El área de trabajo es la parcela, también llamada stand. Ésta debe ser fisiográficamente homogénea; es decir, debe presentar estructura uniforme, cobertura vegetal uniforme, ambiente (topografía, geotopo, exposición, etc.) uniforme (Fig. 3). Así definida, en cada parcela se realizan los inventarios florísticos o relevée (término francés ampliamente empleado).

El tamaño de la parcela está en función de cada ambiente a estudiar, de la heterogeneidad de la vegetación y de la escala. Es conveniente

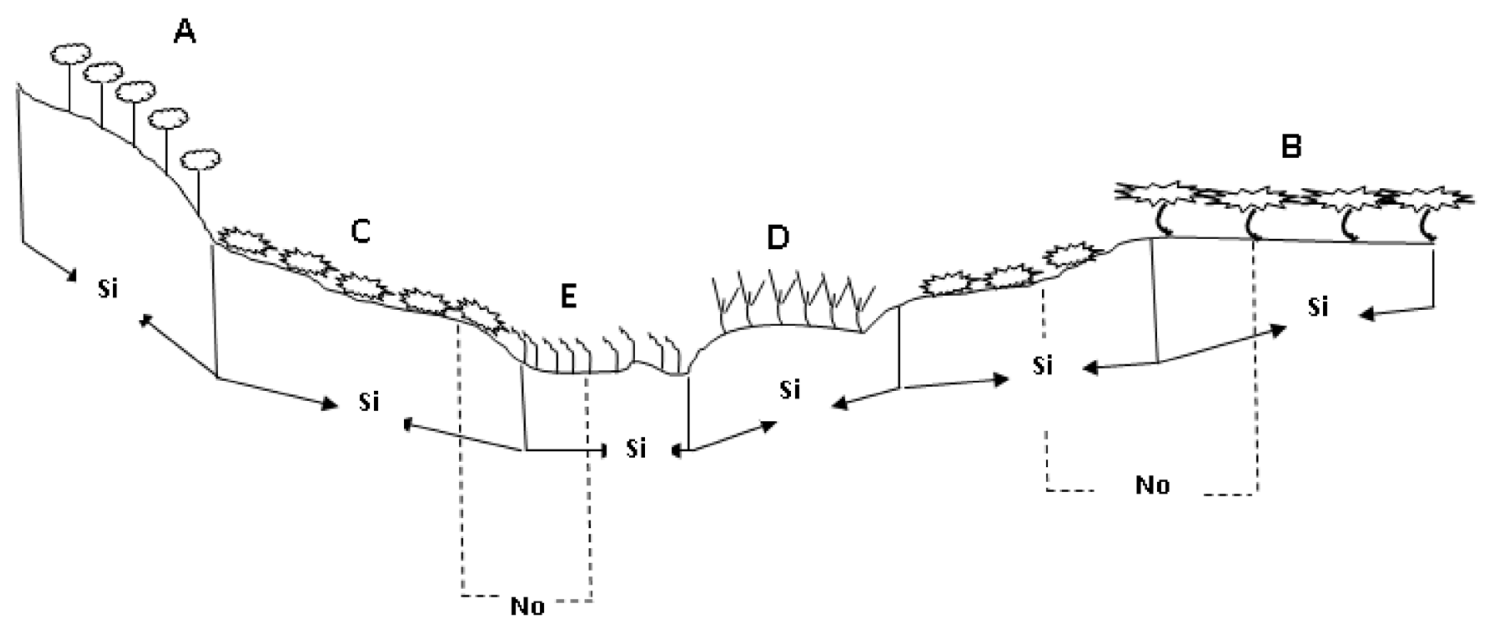

Fig. 3. A, B, C, D y E: comunidades vegetales fisiográficamente homogéneas. Si: ubicación correcta de la parcela (stand), No: ubicación incorrecta. 
realizar la determinación del área mínima, al menos considerando el número de especies. A modo indicativo: para los bosques abiertos es de $200 \mathrm{~m}^{2}$, matorrales pedemontanos áridos de 25 $30 \mathrm{~m}^{2}$, pastizales de valles interandinos de $15 \mathrm{~m}^{2}$, vegas altoandinas de $0,5 \mathrm{~m}^{2}$, etc. La parcela puede ser de diversas formas, cuadrada (en matorrales), rectangular (en vegetación de riberas), circular (fitoplancton en embalses).

El modo o tipo de muestreo es un aspecto siempre sometido a crítica, especialmente considerado subjetivo. Se pueden reconocer al menos tres tipos: Preferencial (o estratificado), Sistemático y al Azar. En el Preferencial, las parcelas se ubican $a$ priori en áreas fisiográficamente diferentes; en el Sistemático, las parcelas se distribuyen siguiendo un modo constante definido en gabinete y en al Azar, las parcelas se distribuyen aleatoriamente (Fig. 4). Para evitar el sobre o sub muestreo de un área, que áreas menores o raras no queden muestreadas, o muestras que incluyan dos áreas fisiográficas diferentes, es conveniente la doble estratificación. Para ello, se puede partir de la determinación de áreas homogéneas mediante fotos aéreas o imágenes satelitales (análisis no supervisado) y luego ajustar a campo cada área a relevar y repetir el análisis sobre la imagen (análisis supervisado). Estratificar, significa dividir el territorio en capas o estratos temáticos: imágenes aéreas o de satélite, litología, pisos de vegetación, etc.

$\mathrm{Al}$ efectuar el inventario o relevamiento se debe decidir qué datos tomar, lo que estará de acuerdo a los objetivos del estudio. Aquí, en parte, surgen las diferencias conceptuales entre las escuelas norteamericana y europea. En la escuela europea, se estima la cobertura de cada especie considerando una escala predeterminada, se incluyen todos los componentes (árboles, arbustos, hierbas, musgos y líquenes), la cobertura total se estima por estrato, se determina cada especie presente y la importancia o dominancia de cada especie está en función a su porcentaje de cobertura. En la escuela norteamericana, se mide el área basal, no suelen incluirse todos los componentes sino centrarse en las leñosas, no tiene prácticamente en cuenta la cobertura por estrato, no tiene en cuenta el nivel específico sino las formas biológicas (árbol, arbusto, hierba) y la importancia o dominancia de los componentes surge del índice del valor de importancia. La escuela europea es adecuada para la cartografía y clasificación de la vegetación y para proponer hipótesis dinámicas, mientras que la norteamericana para el seguimiento de los cambios en la vegetación.

En la práctica, un estudio no se limita a uno que otro método, sino al acoplamiento de diferentes métodos que permitan una mejor comprensión de la realidad bajo análisis. Así, por ejemplo, en una parcela se relevan todas las especies, se determina con precisión y se evalúa cuantitativamente la cobertura específica y por estrato mediante líneas de intercepción. Además se registran y miden todos los parámetros mesológicos considerados relevantes (radiación fotosintéticamente activa, temperatura del suelo y a diferentes alturas según

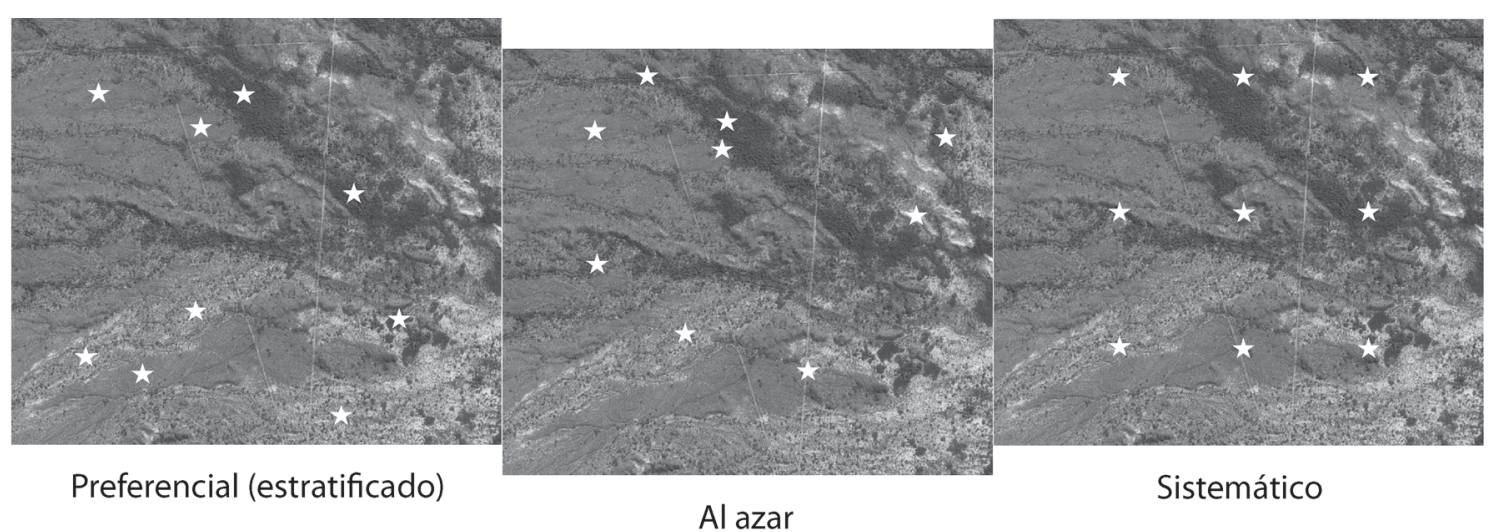

Fig. 4. Tipos de muestreo. Modo de distribución de las parcelas. 


\section{E. Martínez Carretero et al. - Prodromus Sinsistemático y Estudios Fitosociológicos}

estratos, pendiente, textura, $\mathrm{pH}$ y nutrientes del suelo, etc.).

La escala de dominancia (cobertura) empleada en fitosociología es (Fig. 5):

r Cobertura despreciable (individuo aislado o solo) valor medio: 0,1

+ Cobertura muy baja (muy pocos individuos) valor medio: 0,1

1 Cobertura menor del 5\% valor medio: 2,5

2 Cobertura del 5 al 25\% valor medio: 15

3 Cobertura del 25 al $50 \%$ valor medio: 37,5

4 Cobertura del 50 al $75 \%$ valor medio: 62,5

5 Cobertura igual o superior al $75 \%$ valor medio: 87,5

Como estadísticamente ocurre una alta frecuencia de especies cuya dominancia es de orden 2, Westhoff \& van der Maarel (1978) modificaron este nivel dividiéndolo en tres subcategorías:

$2 \mathrm{~m}$ Cobertura próxima al $5 \%$

2a Cobertura del 5 al 15\%

2b Cobertura del 15 al 25\%

El análisis de los datos (inventarios) se realiza comparativamente a través de tablas de relevamientos o matrices de datos.

Inicialmente se incluyen los inventarios relacionados por el tipo de hábitat, piso de vegetación, etc. La tabla inicial es luego reordenada teniendo en cuenta la presencia/ausencia de cada especie (Roig, 1973a). Los relevamientos ocupan las columnas y las especies las filas, de manera que cada relevamiento nuevo se agrega a la derecha de la tabla y cada especie nueva al final de las filas. De esta manera, con cada especie incorporada la tabla crece asimétricamente hacia abajo y a la derecha. Los datos ambientales se incorporan en el encabezado de la tabla y por inventario.

El análisis preliminar de la tabla de inventarios, considerando la presencia/ausencia de cada especie, permite conformar grupos de especies. Cada grupo florístico, teniendo en cuenta los parámetros ambientales, podrá significar una comunidad vegetal.

Para facilitar el análisis de un conjunto grande de datos (inventarios y especies) se elabora la tabla sintética o sinóptica. En ésta, a partir del total de inventarios en cada grupo, se calcula la presencia (o constancia) de cada especie en porcentaje considerando la escala:

Porcentaje de presencia

I $\leq 20 \%$

II $20,1-40 \%$

III $40,1-60 \%$

IV $60,1-80 \%$

V $80,1-100 \%$

En esta tabla sintética habrá tantas columnas como grupos de especies establecidos y todas las especies (filas).
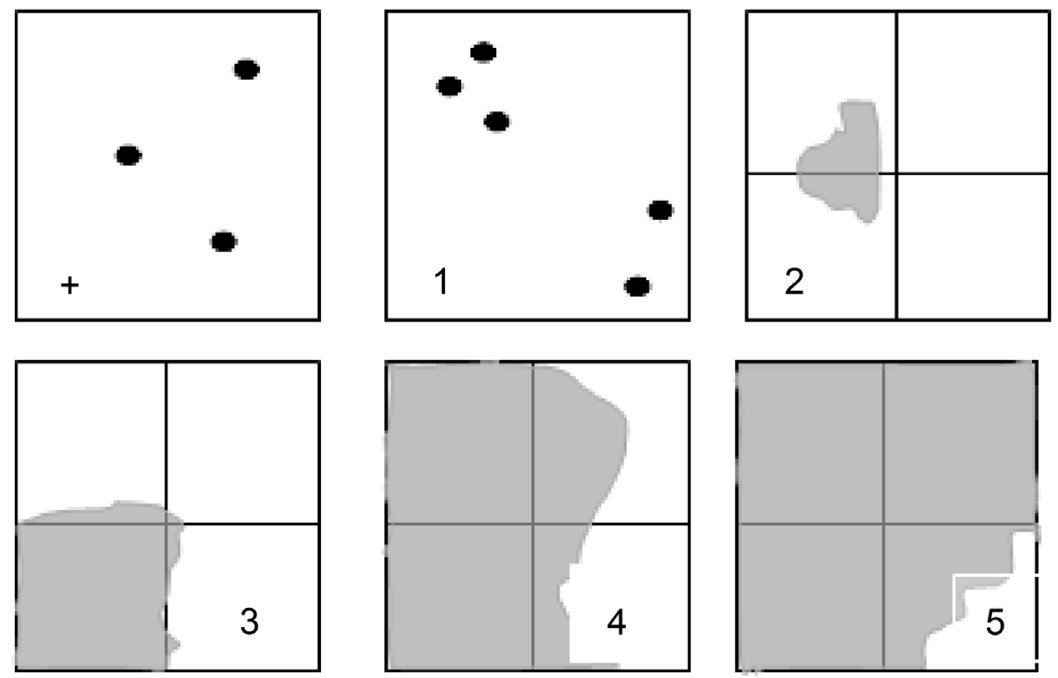

Fig. 5. Escala de dominancia (cobertura). 
La Fidelidad de las especies expresa la mayor o menor restricción de cada especie a la comunidad objeto de estudio. Al comparar diversas comunidades, especialmente en tablas sintéticas o de presencia, surge más claramente el valor de fidelidad de las especies. Se han establecido cinco grados de fidelidad:

5: Especie característica (exclusiva), presente en una sola comunidad vegetal o grupo de comunidades.

4: Especie preferente, aunque se presenta en varias comunidades es más abundante o presenta mejor expresión vegetativa en una comunidad.

3: Especie diferencial, caracteriza a un solo grupo de relevamientos y determinan subasociaciones

2: Especie acompañante, indiferente, ocurre en varias comunidades.

1: Especie accidental, claramente preferente de otra comunidad y ocurre excepcionalmente en la comunidad en estudio.

Al conjunto de especies que permite separar dos o más grupos de inventarios (unidad de vegetación) se las denomina especies diferenciales y suelen indicarse mediante recuadros dentro de la tabla sintética. Las especies diferenciales permiten discriminar entre comunidades pues están presentes con mayor dominancia en una y menor en la otra. También deberían emplearse para reconocer subasociaciones, si se aplica un criterio geográfico, de manera que las especies que marcan geográficamente una raza dentro de una asociación más amplia serían diferenciales geográficas.

Las especies transgresivas son características simultáneamente de dos comunidades (asociaciones) ubicadas en regiones diferentes. Ambas asociaciones son similares florística y ambientalmente.

Una comunidad vegetal resulta en un conjunto de especies propias (caracteristicas), diferenciales y acompañantes que ocupa un hábitat determinado.

El concepto de vegetación potencial es clave en fitosociología pues permite generar modelos predictivos del dinamismo vegetal y organizar el mosaico de tipos de vegetación en un área determinada.

Para un análisis más objetivo y rápido de un conjunto importante de datos (inventarios y especies) se recurre a métodos numéricos de análisis como Agrupamiento (cluster), Componentes Principales,
Factorial, Discriminante, Ordenamiento Polar, etc. comunes en paquetes de análisis de datos como TWINSPAN; VEGANA; DECORANA; PCORD; CANOCO, R, etc.

\section{Análisis sintaxonómico}

Finalmente, si a las comunidades vegetales, sintaxones, se les asigna un nombre de acuerdo con el Código de Nomenclatura Fitosociológica (Barkman et al., 1986; Weber et al., 2000) se debe seguir un conjunto de reglas. Al nombre lo acompaña una descripción mesológica, corológica y una tabla de inventarios. Se debe emplear uno o dos nombres de taxones característicos a los que se añade un sufijo según la categoría sintaxonómica que se trate; el sufijo se añade al segundo taxón empleado (si se emplean dos). Cuando son dos taxones, el primero se modifica mediante una vocal de unión según la declinación del nombre latino. El epíteto específico de la especie utilizada se lo declina a genitivo.

En esta etapa se busca incluir a la comunidad (asociación) en el sistema jerárquico existente, o ajustarlo según corresponda a la nueva información. Para ello, a través del análisis de la composición florística y mesológica se incluirá a la comunidad en unidades superiores como alianza, orden o clase. Cuando el esquema sintaxonómico se publica por primera vez constituye la diagnosis de la asociación.

Cada categoría sintaxonómica se identifica mediante una desinencia: -etum (asociación), -ion (alianza), -etalia (orden), -etea (clase). Por ejemplo, en la vegetación de la Payunia: Sarcocornietea perennis (clase) (Comunidades arbustivas halófilas), Sarcocornietalia perennis (orden), Sarcocornion perennis (alianza), SuaedoSarcocornietum, Frankenio-Sarcocornietum y Puccinellio-Sarcocornietum (asociaciones).

De esta manera, un ordenamiento sintaxonómico representa la distribución de las especies según su amplitud ecológica. Las de menor amplitud (características) se restringen a una asociación; las de alianza, de amplitud mayor, ocurren en al menos dos asociaciones ecológicamente próximas lo mismo sucede con las de orden y clase. Esquemáticamente sería (Fig. 6):

Estudiar fitosociológicamente las comunidades vegetales requiere conocer su composición florística y su sistematización (sintaxonomía), las 
E. Martínez Carretero et al. - Prodromus Sinsistemático y Estudios Fitosociológicos

Grupo de

relevamientos/Especies $\quad 1 \quad 2$

34

5

6

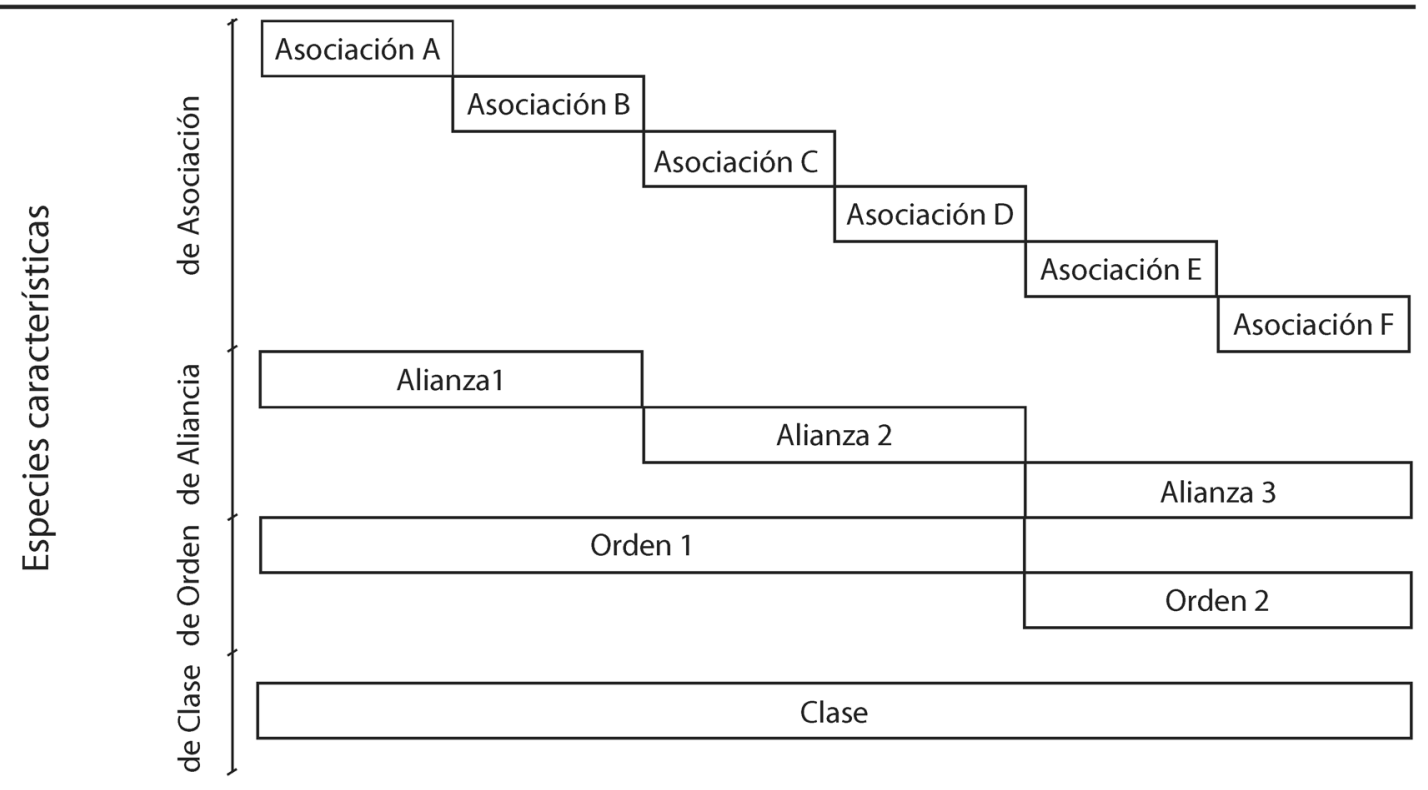

Fig. 6. Esquema de ordenamiento sintaxonómico.

condiciones mesológicas (geoformas, clima, suelos, acciones antrópicas), su distribución (corología, fitogeografía), sus dinamismos (naturales y antrópicos). De ello resultan valiosos aportes para el inventario de recursos naturales (vegetales), estudios de paisaje vegetal, recuperación de ambientes degradados, conservación de la biodiversidad, cartografía vegetal, planes de manejo de áreas protegidas, ordenamiento territorial, evaluación de impacto, agricultura, silvicultura, urbanismo (urbataxon: Claisse, 1977 y Claisse \& Géhu, 1978), entre otros.

De manera sintética en la Tabla 5 se presentan los diferentes enfóques, y los principales componentes de cada uno, que se tienen en cuenta al estudiar la vegetación de un área.

\section{La Fitosociología en los Estudios de Vegetación}

Como en toda rama del conocimiento en activa evolución, la discusión entre Fitosociología y Ecología Vegetal, enfoque inductivo y deductivo, subjetividad y objetividad, etc. representa diferentes aproximaciones en el campo de la ciencia de la vegetación.
Importantes ajustes/modificaciones al método tradicional sigmatista se han efectuado en las últimas décadas principalmente por Duvigneaud (1946), Ellenberg (1956), Mayor (1995), Rivas-Martínez (1987, 2005), quienes considerando la composición florística y factores ecológicos llegan a los grupos ecológicos. Sin embargo, fue posiblemente Poore (1955) quién analizó las deficiencias metodológicas y destacó los importantes aportes positivos. El aporte de Duvigneaud (1946) sobre el valor de los grupos ecológicos, o socioecológicos, para definir una asociación (comunidad) es relevante. Ellenberg (1988) recurre a la autoecología de las especies para analizar la respuesta de éstas a los factores ambientales y así establecer los grupos ecológicos y luego emplearlos, combinándolos, para establecer las unidades de vegetación. Posteriormente, MuellerDombois \& Ellenberg (1974) siguieron criterios florísticos y ecológicos (factores ambientales) para establecer unidades de vegetación. Para Ellenberg (1954), es la alianza en la que adquiere valor el criterio de especie característica. Poore (1962), trabajando en fitosociología y, a su vez, tomando una postura crítica llegó a proponer el método de aproximaciones sucesivas en ecología 


\begin{tabular}{|c|c|}
\hline 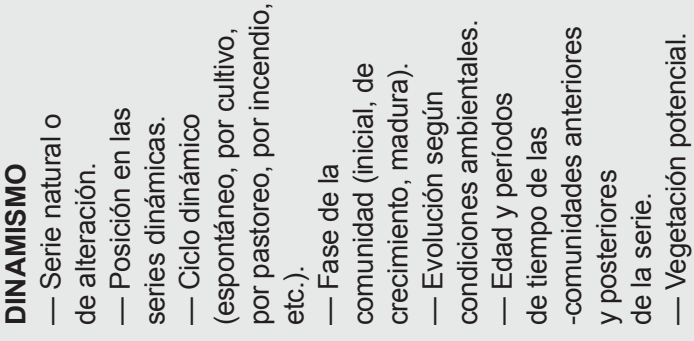 & 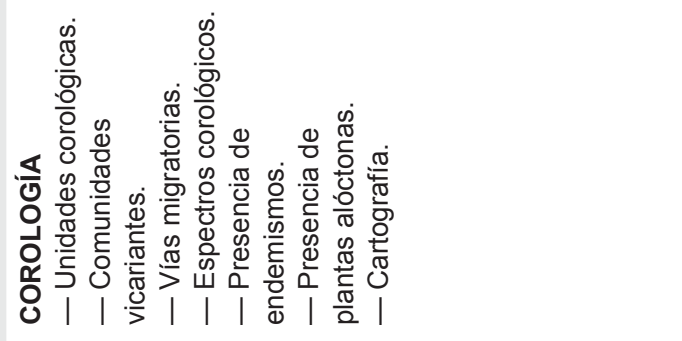 \\
\hline 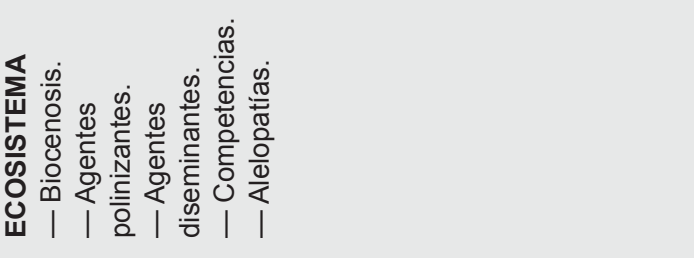 & 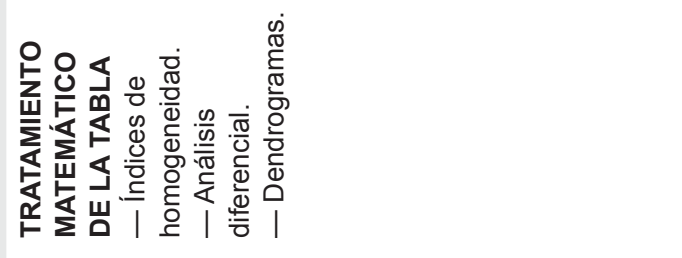 \\
\hline 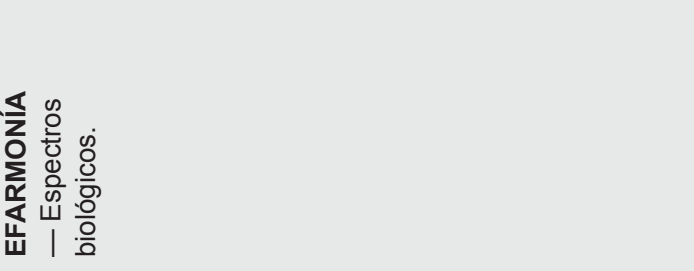 & 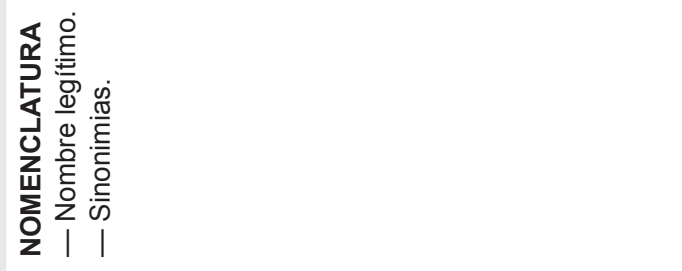 \\
\hline 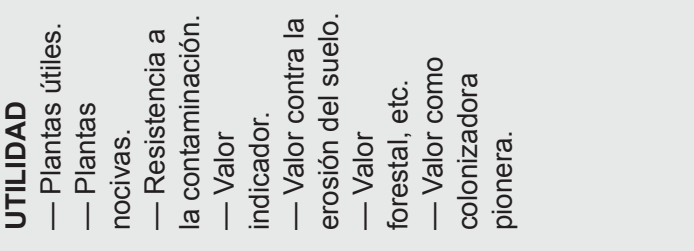 & 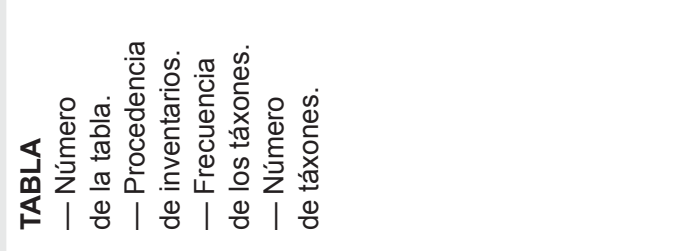 \\
\hline 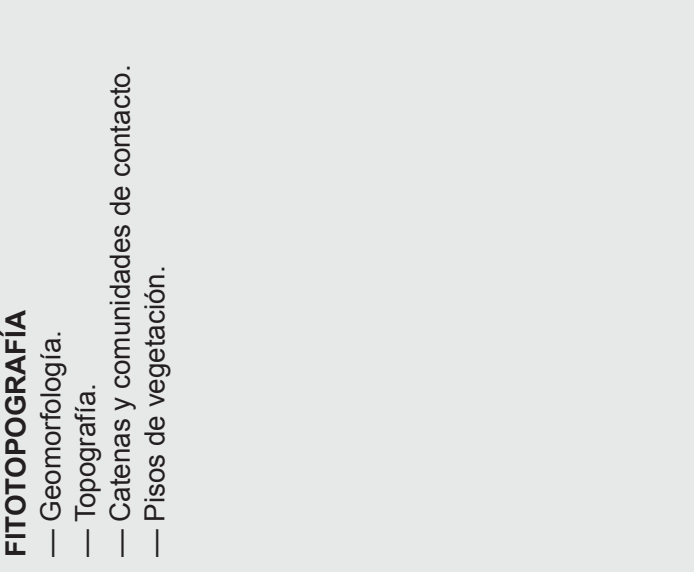 & 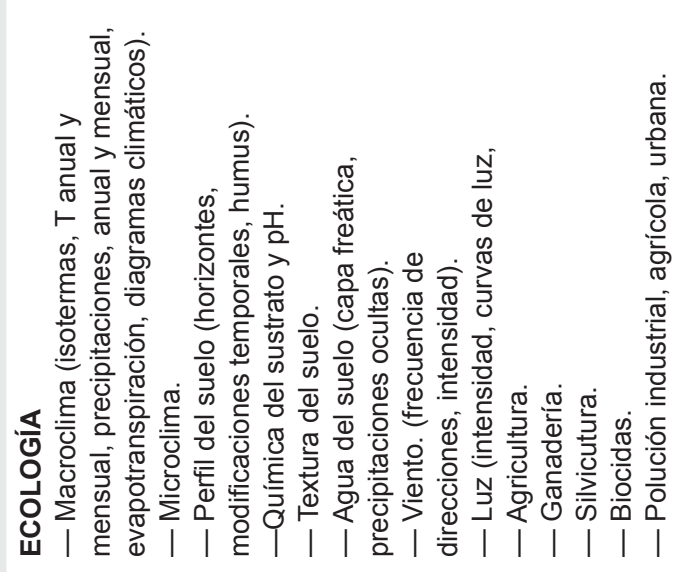 \\
\hline 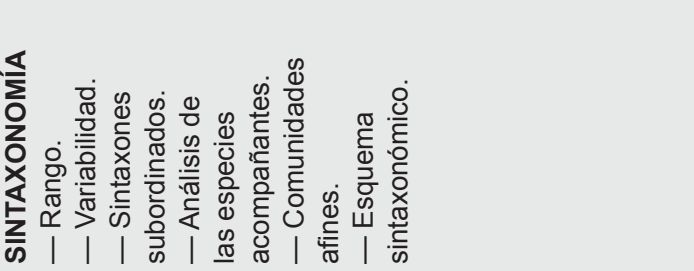 & 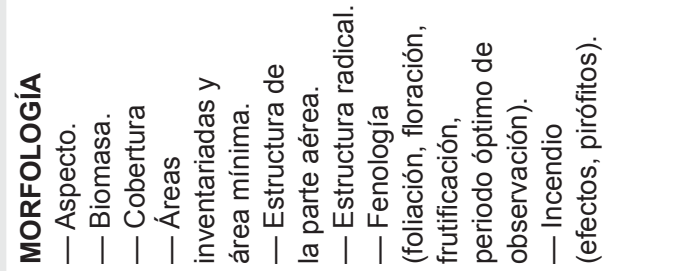 \\
\hline
\end{tabular}




\section{E. Martínez Carretero et al. - Prodromus Sinsistemático y Estudios Fitosociológicos}

descriptiva, útil para la diagnosis de los factores ecológicos que diferencian una comunidad de otra, no aceptando a la fidelidad como criterio de clasificación. Finalmente, Rodwell (1991) destaca el valor importante de la fitosociología en el muestreo meticuloso de los hábitats y el empleo de esos datos para estudiar problemas ecológicos.

Mientras la fitosociología busca detectar modelos de vegetación y describir las comunidades vegetales y su relación con parámetros ambientales, la ecología vegetal examina los procesos que determinan la variación en la abundancia de las especies a lo largo de un gradiente ( $\varnothing$ kland, 1990; Ewald, 2003); de esta manera, en la fitosociología se reconoce la variación de la vegetación como respuesta a la variación en factores ecológicos y trata de describir la vegetación. Además, ante un complejo de comunidades afines el método permite formular diversas hipótesis acerca de las relaciones ecológicas entre ellas. Resulta así en una ciencia integradora donde la respuesta son las asociaciones y los factores son las variables que las condicionan (edáficas, gemorfológicas, florísticas/ históricas y de uso, entre otras). El concepto de comunidad vegetal basado en la idea de Clements (1916) y en la de continuum de Gleason (1939) polarizó la discusión por más de 30 años. Por otra parte, la postura: independencia entre el observador y la muestra, opuesta a la de muestra subjetiva de la fitosociología, ha contribuido significativamente a la larga discusión. Finalmente, el marco epistemológico aporta para interpretar si sólo se trata de un cambio de paradigma.

El empleo de imágenes satelitales y la aplicación del concepto de land unit (homogeneidad fisiográfica) del CSIRO australiano contribuyen a una mejor objetivación al seleccionar las áreas a muestrear (relevar), permitiendo su réplica por diversos investigadores. El empleo de técnicas de clasificación y ordenamiento de datos provee mayor rapidez y objetividad en la definición de las comunidades y también permite la visualización de patrones de variación de grandes conjuntos de datos florísticos. Un paso importante está dado por la Geosinfitosociología, con una visión más amplia e integradora de la relación vegetacióngeomorfología-bioclima, llegando al Paisaje vegetal.

En síntesis: En estudios intensivos, de detalle, a nivel local, de la vegetación se puede prescindir de la sistematización de las unidades; sin embargo, a escalas mayores es conveniente incluirlas en un esquema jerárquico.

La información generada y acumulada en estudios fitosociológicos (florísticos-ecológicos) aporta significativamente al conocimiento de la distribución y rango de tolerancia a factores ambientales de las especies y las comunidades. De esta manera, las diferencias en composición florística están ligadas a cambios temporales o espaciales en el hábitat.

El análisis comparativo de tablas florísticas, considerando las especies características y diferenciales, permite fomar grupos ecológicos (socioecológicos), vinculando además las técnicas florísticas con las de ecología vegetal.

La cartografía fitosociológica y la elaboración de una base de datos florísticos a nivel regional o nacional permite generar una base sólida para programas de conservación, determinación de habitats, meta-análisis (cartografías predictivas), asociado a datos biogeográficos (atlas florísticos) resultan patrones ecológicos a diferentes escalas.

\section{Biogeografía}

La República Argentina por su ubicación geográfica al sur del continente, estar prácticamente rodeada de mar y el dominio del sistema andino en toda su extensión norte-sur, presenta una importante diversidad climática, edáfica y de vegetación. La sombra de lluvias en la vertiente oriental andina determina la Diagonal Árida Argentina con desiertos fríos y cálidos y regímenes de lluvia mediterráneo y tropical (Martínez Carretero, 2013). En el nor-noreste la influencia del Anticiclón del Atlántico concentra las precipitaciones en verano con clima subhúmedo y húmedo.

Esta diversidad clima-relieve-vegetación determina la presencia de trece provincias fitogeográficas reunidas en cuatro dominios (Cabrera, 1976).

La tesela constituye la unidad elemental mediante la cual se define un espacio geográfico, variable en extensión, ecológicamente (fisiográficamente) homogéneo que contiene un solo tipo de vegetación potencial y una sola secuencia de comunidades de sustitución. El sigmetum es la unidad tipológica, y expresa el conjunto de comunidades vegetales o estadíos resultados de la sucesión incluyendo las características geográficas, mesológicas y florísticas 
de la asociación madura o estable. La fitosociología dinámico-catenal y la información sintaxonómica de extensos territorios constituyen el criterio básico para establecer unidades biogeográficas.

Para Rivas Martínez et al. (1985, 2011) las unidades biogeográficas son: Área, Distrito, Sector, Provincia, Región y Reino. El Área posee abundantes unidades de paisaje, especies, asociaciones y un sigmeta topográfico. El Distrito, series, geoseries y geoclinoseries. El Sector, grupo de Distritos, asociaciones y series de vegetación propia. La Provincia, macroseries y geomacroseries (pisos altitudinales). La Región se integra por un grupo de provincias. El Reino es la unidad mayor, generalmente pluricontinental. Estos autores, basados en datos bioclimáticos y florísticos, proponen las siguientes unidades biogeográficas para Argentina:

Reino Neotropical-Austroamericano

Subreino Neotropical, Superregión ChacoBrasileña

Región Chaco-Brasileña: Provincia Paranaense

Región Chaqueña: Provincia Chaqueña Septentrional, Provincia Chaqueña Meridional

Superregión Surandino Tropical, Región Surandina Tropical: Provincia Puneña Xerofitica, Provincia Boliviano-Tucumana, Provincia Yungueña.

Subreino Austro Americano

Región Pampeana: Provincia Pampeana Mesofítica, Provincia Pampeana Xerofítica

Región Mesochilena-Patagónica: Provincia Andino Mediterrána, Provincia Monte Argentino, Provincia

Patagónica Septentrional, Provincia Patagónica Meridional

Región Valdiviana-Magallánica: Provincia Valdiviana, Provincia Magallánica Templada, Provincia Austromagallánica Boreal, Provincia Islas Malvinas

Subreino Circumantarctico

Región Antártica Insular: Provincia Islas AntárticasAtlánticas

Región Antártica Continental: Provincia Antártico Occidental

En este Prodromus se sigue el esquema fitogeográfico propuesto por Cabrera (1971, 1976), ajustado en diferentes regiones, entre otros, por Prado (1993a,b), Martínez Carretero (1995, 2005), Roig (1998) y Fontana (1998).

\section{PRODROMUS}

\section{SINTAXONOMÍA}

Hasta el momento se citan 57 clases de vegetación para la República Argentina y la presencia posible de otras 16 clases, distribuidas en todas las regiones fitogeográficas (Fig. 7).

I.- Alnetea acuminatae Galán de Mera et Orellana 2006

Alisedas andinas acompañadas de arbustos escandentes y lianas. Bosques deciduos del $\mathrm{S}$ de Bolivia y $\mathrm{N}$ de Argentina (1500-2000m).

Alnetalia acuminatae idem auctores 2006

Pruno tucumanensis-Alnion acuminatae Aceñolaza 1996

Polysticho montevidensis-Alnetum acuminatae Aceñolaza 1996 (Fig.11g)

II.- Ambrosietea elatioris Eskuche et Iriart 1996a, b Vegetación peridoméstica del norte de Argentina.

Amaranthetalia viridis Eskuche et Iriart 1996a, b Rumicion obovati Eskuche et Iriart 1996a, b Parthenio-Amaranthion hybridonis Iriart 2016

Rumicenion obovati Iriart 2016

Rumicetum obovati Eskuche et Iriart 1996a, b Rel. 1 Amarantho viridis-Coronopodetum didymi Eskuche et Iriart 1996a, b Rel. 2

Eleusino-Cynodontetum Eskuche et Iriart 1996 a, b Rel. 3

Xanthietum strumarii Iriart 2016

Parthenietum hysterophori Iriart 2016

Leonuretum japonici Iriart 2016

Amaranthetum viridi-hybridonis Iriart 2016

Sennetum occidentalis Iriart 2016

Pileo microphyllae-Pteridetum vittatae Eskuche et Iriart 1996a, b el. 4

Chenopodienion hircini Iriart 2016

Urochloo plantaginei-Gomphrenetum bolivianae Iriart 2016

Polygonetalia punctati Eskuche 2004

Polygono punctati-Rumicion paraguayense Fontana 1991a

Polygono punctati-Rumicetum paraguayensis Fontana 1991a, Eskuche 2004

Apio sellowiani-Ambrosietum elatioris Eskuche 2004

Polygono punctati-Melantheretum latifoliae Eskuche 2004 prov. 


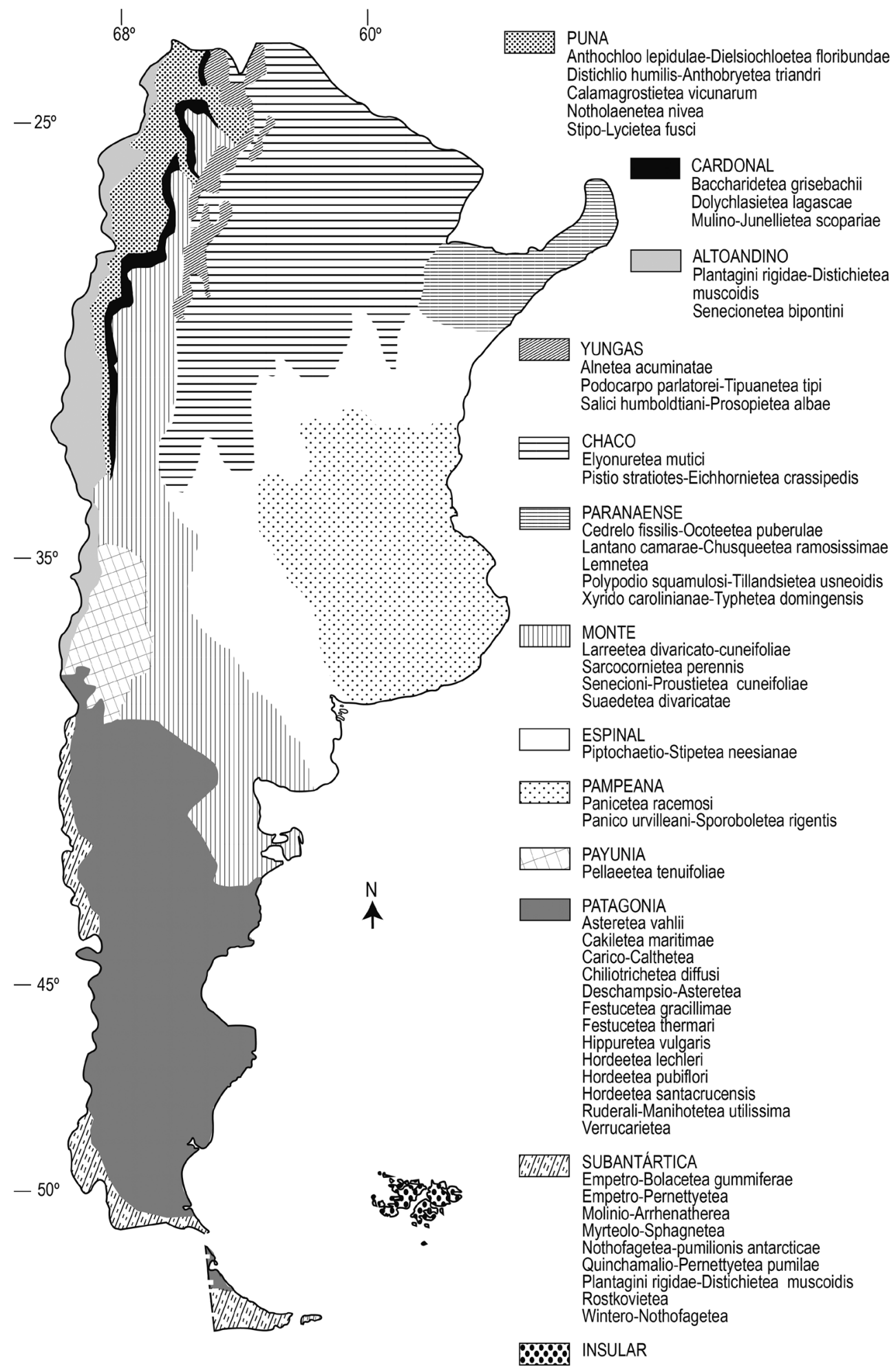

Fig. 7. Unidades sintaxonómicas y regiones fitogeográficas. 
Alternanthero-Eragrostion hypnoidis Eskuche 2004

Lindernio-Mecardonietum herniaroidis Eskuche 1975, 1979

Alternanthero-Eragrostietum hypnoidis Eskuche et Fontana 2004 (in Eskuche 2004)

\section{III.- Anthochloo lepidulae-Dielsiochloetea}

floribundae Rivas Martínez et Tovar 1982

Vegetación andina dispersa del límite superior en pedregales y laderas con crioturbación de la Puna, en Perú, Bolivia y norte de Argentina y Chile.

Anthochloo lepidulae-Dielsochloetalia

floribundae Rivas Martínez et Tovar 1982

Wernerion pseudodigitatae Ruthsatz 1977, prov. Nototricho auricomae-Chaetantheretum sphaeroidalis Navarro 1993

IV.- Artemisietea vulgaris Lohmeyer et al. ex von Rochow 1951 (Laníková 2009)

Vegetación antrópica rica en cardos en suelos nitrogenados.

Onopordetalia acanthii Br. Bl. et Tüxen 1951 ex

Klika in Klika \& Hadač 1944 (Bardat et al. 2004)

Carduetum nutantis macrocephali Méndez 1983, em.

V.- Asteretea vahlii Eskuche 2005

Vegetación litoral de los lagos patagónicos y de los canales magallánicos.

Eleocharitalia pachycarpae Eskuche 2005

Senecionion zosterifolii Eskuche 2005

Arenario-Azorelletum trifoliolate Eskuche 2005 Rel. 5

Carici-Senecionetum zosterifolii Eskuche 2005 Rel. 6

Senecioni zosterifolii- Littorelletum australis

Eskuche 2005 Rel. 7

Crassulo-Limoselletum lineatae Eskuche 2005 Rel. 8

Downingio-Isoetetum savatieri Eskuche 2005 Rel. 9

Hydrocotylo-Juncetum arctici Eskuche 2005, prov.

Leptinelletalia scariosae Eskuche 2005

Colobantho-Plantaginion barbatae Eskuche 2005

Colobantho-Plantaginetum barbate Eskuche 2005

Rel. 10

Senecionion smithii Eskuche 2005

Senecioni smithii-Poetum robustae Eskuche 2005

Rel. 11

Caricetum darwinii Eskuche 2005 Rel. 12

VI.- Baccharidetea grisebachii Roig et Martínez Carretero 1998
Vegetación riparia de ríos temporarios de los Andes áridos.

Baccharidetalia grisebachii Roig et Martínez Carretero 1998

Baccharidion grisebachii Roig et Martínez Carretero 1998

Baccharidetum grisebachii Roig et Martínez Carretero 1998 Rel. 13

Sintypus: relevé 10 (Tab. 72)

Discarietum trinervis Martínez Carretero 2000 Rel. 14 Holotypus: relevé 101 (Tab. 6)

VII.- Calamagrostietea vicunarum Rivas Martínez et Tobar 1982, Gutte 1985

Vegetación de pajonales y tolares altoandinos de Perú, Bolivia y norte de Argentina y Chile.

=Luzulo racemosae-Calamagrostietea vicunarum Gutte 1980, 1988

Parastrephietalia lepidophyllae Navarro 1993

Parastrephion lepidophyllae Navarro 1993

Muehlenbergio fastigiatae-Parastrephietum lepidophyllae Navarro 1993

Acantholippio salsoloidis-Lampayetum castellani Navarro 1993

Lobivio ferocis-Fabianion densae (Ruthsatz 1977) Navarro 1993

Lobivio pentlandii-Fabianetum densae Navarro 1993

Gutierrezio gilliesii-Junellietum seriphioidis (Navarro 1993), nom. mut. prop.

Basiónimo: Gutierrezio gilliesii-Verbenetum seriphioides Navarro 1993

Urbanio pappigerae-Stipion frigidae Navarro 1993

Stipo frigidae-Senecionetum puchii Navarro 1993

Fabiano bryoidis-Stipetum venustae Navarro 1993

Fabiano bryoidis-Stipetum venustae Navarro 1993

Fabiano bryoidis-Stipetum venustae Senecionetosum puchii Navarro 1993

Lobivio-Fabianion densae Ruthsatz 1977 (in Navarro 1993)

Fabianion densae Ruthsatz 1977, nom. prov. inval. (in Navarro 1993)

Urbanio papigerae-Stipion frigidae Navarro 1993

Jaravetum absconditae Martínez Carretero, Dalmasso, Márquez et Martinelli 2009 Rel. 15

Sintypus: relevé 40 (Tab. 7)

Stipion ichu Seibert et Menhofer 1991, 1992

VIII.- Cakiletea maritimae Tüxen et Preisig in Tüxen 1950 


\section{E. Martínez Carretero et al. - Prodromus Sinsistemático y Estudios Fitosociológicos}

Vegetación en cúmulos de residuos orgánicos de resaca en playas marinas.

Cakiletalia maritimae Tüxen ex Oberdorfer 1949;

Tüxen in Br.Bl. et Tüxen 1952 (art. 31)

Atriplicion littoralis Nordhagen 1940 (In: Tüxen 1950)

Senecionetum candidantis Reška., s/f

IX.- Carico-Calthetea Roig, Anchorena, Dollenz, Faggi et Méndez 1985

Turbera minerotrófica densa, rica en ciperáceas, incluye comunidades edáficas de suelos húmedos

de Patagonia y Tierra del Fuego.

Carico-Calthetalia Roig, Anchorena, Dollenz, Faggi et Méndez 1985 (T)

Carico-Calthion ídem auctores 1985 (T)

Calthetum sagittatae ídem auctores (T) Rel. 16

Lectotypus: TBPA relevé 3 (pág. 448)

Cortaderietum piloso-minimae Gandullo et Faggi 2003 Rel. 17

Typus : Rel. 262

Caltho sagittatae-Juncus scheuchzerioides Gandullo et Faggi 2003 Rel. 18

Typus : Rel. 93

Gunnero magellanicae-Calthetum sagittatae Gandullo et Faggi 2003 Rel. 19

Typus. Rel. 16

Cortaderietum pilosae-minimae Gandullo et Faggi 2003

Juncion lesueurii Gandullo et Faggi 2005

Carico gayanae-Eleocharetum melanostachyo Gandullo et Faggi 2005

Alopecuretosum aequalis Gandullo et Faggi 2005

Carico gayanae-Eleocharetum albibracteatae

Gandullo et Faggi 2005

Festucetum scabriusculae Gandullo et Faggi 2005

Azorello trifoliolatae-Plantagetum uniglumitis Gandullo et Faggi 2005

X.- Cedrelo fissilis-Ocoteetea puberulae Oriol de Bolós, A., C. Cervi et G. Hatschbach 1991

Reune diferentes selvas, higrófilas y mesófilas del sur de Brasil, Misiones, etc.; bordeando el valle del río Paraná entre los departamentos de Misiones (Argentina) e Itapuá (Paraguay)

Piptadenio rigidae-Nectandretalia megapotamicae idem auctores 1991

Piptadenia rigidae- Nectandrion megapotamicae idem auctores 1991

Piptadenio rigidae-Nectandretum megapotamicae idem auctores 1991
XI.- Chiliotrichetea diffusi Galán de Mera et Orellana 2006

Matorrales heliófilos, etapa primaria de sustitución de lengares y ñirantales argentinos y chilenos.

Chiliotrichetalia Roig, Anchorena, Dollenz, Faggi et Méndez 1985

Chiliotrichion ídem auctores 1985

Elymo-Chiliotrichetum ídem auctores 1985 Rel. 20=

Lectotyopus TBPA, relevé 107 (pág. 385)

Anemono-Chiliotrichetum ídem auctores $1985 \underline{\text { Rel. }} 21$

Lectotipus: TBPA, relevé 50 (pág. 385)

Embothryo-Pernettyetum mucronatae ídem auctores 1985 Rel. 22

Lectotypus: TBPA, relevé 2 (pág. 385)

Senecioni-Adesmietum boronioidis Méndez et Ambrosetti 1985 Rel. 23

Lectotypus: TBPA, relevé 119 (pág. 664)

Stipion brevipedes Roig, Anchorena, Dollenz, Faggi et Méndez 1985

Stipo Mulinetum ídem auctores 1985 (T) Rel. 24

Lectotypus: TBPA, relevé 4 (pág. 385)

Stipo-Senecionetum patagonici ídem auctores 1985 , em. Rel. 25

Lectotypus: TBPA, relevé 20 (pág. 385)

XII.- Deschampsio-Asteretea Roig, Dollenz et Méndez 1985

Vegetación riparia marina y fluvial magallánica del sur de Argentina y Chile.

Deschampsio-Asteretalia Roig, Dollenz et Méndez 1985 (T)

Deschampsio-Asterion ídem auctores 1985 (T)

Plantago-Deschampsietum ídem auctores 1985 (T) Rel. 26

Lectotypus: TBPA relevé 156 (pág. 478).

Plantagini-Deschampsietum laxae Eskuche 2005 n. $\mathrm{n}$.

Apio-Senecietum smithii ídem auctores 1985 Rel. 27

Lectotypus: TBPA relevé 46 (pág. 478)

Deschampsietum kingii ídem auctores 1985, val. Eskuche 2005 Rel. 28

Lectotypus: TBPA relevé 108 (pág. 478)

Arenario-Senecionetalia Roig, Dollenz et Méndez 1985, em.

Arenario-Senecionion Roig, Dollenz et Méndez 1985

Arenario-Elymetum ídem auctores 1985, em. Rel. 29

Lectotypus: TBPA, relevé 19 (pág. 478)

Luzulo-Ourisietum idem auctores 1985 Rel. 30

Lectotypus: TBPA relevé 148 (pág. 478) 


\section{XIII.- Distichlio humilis-Anthobryetea triandri} Navarro 1993

Vegetación andina halófila de cuencas endorreicas de altiplanos puneños de Argentina, Chile, Bolivia y sur de Perú.

Anthobryetalia triandri Navarro 1993

Sarcocornion pulvinatae Ruthsatz ex Navarro 1993

Atriplici-nitrophiloidis-Sarcocornietum pulvinatae Navarro 1993

Atriplici-nitrophiloidis Sarcocornietum triglochinetosum Navarro 1993

Muehlenbergio fastigiatae-Distichlietum humilis Navarro 1993

Parastrephia lucida Ruthsatz, prov.

XIV.- Dolychlasietea lagascae Roig et Martínez Carretero 1998

Vegetación saxícola preandina.

Dolychlasietalia lagascae Roig et Martínez Carretero 1998

Dolychlasion lagascae Roig et Martínez Carretero 1998

Dolychlasietum lagascae prov. Roig et Martínez Carretero 1998 Rel. 31 (Fig. 10j)

Mutiesietum linifoliae Roig et Martínez Carretero 1998 Rel. 32

XV.- Elyonuretea mutici Eskuche 1992a

Pajonales mesófilos seminaturales del NE de la Argentina $\mathrm{y}$ regiones adyacentes.

Pfaffio tuberosae-Elyonuretalia mutici Eskuche 1992a

Melanantho-Axonopodion Eskuche et Fontana 1996b

Vegetación de las islas argentinas del Alto Paraná: comunidades del campo abierto.

Melanantho-Axonopodetum fissifolii Eskuche et Fontana 1996b Rel. 33

Elyonuro mutici-Aristidion jubatae Fontana 1996

Ocimo neurophylli-Elyonuretum mutici Fontana 1996 Rel. 37

Macrosiphonio undulatae-Aristidetum jubatae Fontana 1996 Rel. 34 (Fig. 11d)

Stevio lundianae-Aristidetum jubatae Fontana 1996

Diplothemio campestris-Axonopodetum suffulti

Fontana 1996 (Fig. 11c)

Diplothemio campestris-Axonopodetum suffulti eryngietosum Fontana 1996

Diplothemio campestris-Axonopodetum suffulti aspicarpetosum Fontana 1996
Stevio entrerriensis-Elyonurion mutici Eskuche 1992a (Fig. 11f)

Crotono-Elyonuretum mutici Eskuche 1992a Rel. 38

Stipo nutantis-Elyonuretum mutici Eskuche 1992c

Ayenio apricae-Elyonuretum mutici Eskuche 1992a

Schlechtendalio luzulaefoliae-Brometum auletici

Eskuche 1992a (Fig. 11j)

Heimio salicifoliae-Elyonuretalia mutici Eskuche 1992a

Fimbristylido castaneae-Elyonurion mutici

Eskuche 1992a

Turnero grandiflorae-Elyonuretum mutici Eskuche 1992c Rel. 39

Galactio longifoliae-Elyonuretum mutici Eskuche 1992a

Gaillardio megapotamicae-Elyonurion mutici Eskuche 1992a

Passifloro mooreanae- Elyonuretum mutici Eskuche 1992a

Bothriochloo barbinodis- Elyonuretum mutici Eskuche 1992a

Paspalo urvillei-Elyonuretum mutici Eskuche 1992a

XVI.- Empetro-Bolacetea gummiferae Roig, Anchorena, Dollenz, Faggi et Méndez 1985, em.

Asociaciones de plantas criófilas patagónicas y fueguinas del sur argentino y chileno.

Berberido-Empetretalia rubri Roig, Anchorena,

Dollenz, Faggi et Méndez 1985, em.

Berberido-Empetrion rubri ídem auctores 1985, em.

Berberido-Empetretum rubri ídem auctores 1985, em. Rel. 40

Lectotypus: TBPA 7 (pág. 394)

Adesmio-Empetretum rubri ídem auctores 1985, em. Rel. 41

Lectotypus: TBPA relevé 18 (pág. 394)

Carici-Bolacetum gummiferae ídem auctores 1985, em. Rel. 42

Lectotypus: TBPA relevé 25 (pág. 394).

Hamadryo-Oreopoletalia glacialis Roig, Anchorena, Dollenz, Faggi et Méndez 1985, em.

Vegetación del desierto andino.

Hamadryo-Oreopolion glacialis $i$ dem auctores 1985, em.

Leucherio-Nassauvietum juniperinae ídem auctores 1985, em. Rel. 43

Lectotypus: TBPA relevé 64 (pág. 394)

Adesmio-Perezietum megalanthae ídem auctores 1985, em. Rel. 44

Lectotypus: TBPA relevé 30 (pág. 394)

Onuro-Perezietum Méndez et Ambrosetti 1985 Rel. 45 


\section{E. Martínez Carretero et al. - Prodromus Sinsistemático y Estudios Fitosociológicos}

Lectotypus: TBPA relevé 85 (pág. 667)

Bolaco-Empetretum rubri idem auctores 1985, em. Rel. 46

Lectotypus: TBPA relevé 55 (pág.667)

Gunnero-Marsippospermetum grandiflori ídem auctores 1985 Rel. 47

Lectotypus: TBPA relevé 87 (pág. 665)

Bolaco-Marsippospermetum grandiflori ídem auctores $1985 \underline{\text { Rel. }} 48$

Lectotypus: TBPA relevé 26 (pág. 665)

Bolaco-Violion tridentatae Roig, Anchorena, Dollenz, Faggi et Méndez 1985, em.

Violo-Abrotanelletum emarginatae ídem auctores 1985, em. Rel. 49

Lectotypus: TBPA relevé 32 (pág. 394)

XVII.- Empetro-Pernettyetea Oberdorfer 1960

Vegetación arbustiva, rica en ericáceas.

Pernettyetalia Oberdorfer 1960

Festuco-Pernettyion Oberdorfer 1960, prov.

Festuco Pernettyetum Oberdorfer 1960, prov.

Blechno-Pernettyion mucronatae Oberdorfer 1960

Blechno-Pernettyetum mucronatae Oberdorfer 1960

Empetro-Pernettyietum mucronatae Oberdorfer 1960

XVIII.- Festucetea gracillimae Roig, Anchorena, Dollenz, Faggi et Méndez 1985

Estepa magallánica del sur de Santa Cruz y norte del Tierra del Fuego.

Nardophyllo-Festucetalia gracillimae ídem auctores 1985, em.

Nardophyllo-Festucion gracillimae ídem auctores 1985, em.

Festucetum gracillimae idem auctores 1985 (T) Rel. 50 (Fig. 11a)

Lectotypus: TBPA relevé 53 (pág. 404)

Elymo-Festucetum gracillimae ídem auctores 1985, nom. mut. Rel. 51

Lectotypus: TBPA relevé 72 (pág. 404)

(Basiónimo: Agropyro-Festucetum gracillimae ídem autores 1985

Junellio-Festucion gracillimae Roig, Anchorena,

Dollenz, Faggi et Méndez 1985, nom. mut.

Basiónimo Verbeno-Festucion gracillimae ídem auctores 1985

Junellio-Festucetum gracillimae ídem auctores 1985, nom mut. Rel. 52

Basiónimo Verbeno- Festucetum gracillimae ídem auctores 1985

Lectotypus: TBPA relevé 16 (pág. 404) (T)
Stipo-Nassauvion ulicinae Roig, Anchorena, Dollenz, Faggi et Méndez 1985

Stipo-Nassauvietum ulicinae ídem auctores 1985 (T) Rel. 53

Lectotypus: TBPA relevé 26 (pág. 409)

Berberido-Junellietum tridentis ídem auctores 1985, nom. mut. Rel. 54

Basiónimo: Berberido-Verbenetum tridentis ídem auctores 1985

Lectotypus: TBPA relevé 7 (pág. 409)

Valeriano-Mulinetum spinosi ídem auctores 1985

Rel. 55

Lectotypus: TBPA relevé 32 (Pág. 409)

Gutierrezio-Stipetum ameghinoi ídem auctores 1985

Rel. 56

Lectotypus: TBPA relevé 146 (pág. 409)

Polemonio-Adesmietum boronioidis ídem auctores 1985 Rel. 57

Lectotypus: TBPA relevé 180 (pág. 423)

Gamochaeto-Festucetalia gracillimae Roig,

Anchorena, Dollenz, Faggi et Méndez 1985, em.

Gamochaeto Festucion gracillimae Roig, Anchorena, Dollenz, Faggi et Méndez 1985 (T)

Geranio-Festucetum gracillimae ídem auctores 1985

(T) Rel. 58

Lectotypus: TBPA, relevé 52 (pág. 427)

Dactylo-Festucetum gracillimae $i$ dem auctores 1985

Rel. 59

Lectotypus: TBPA, relevé 9 (pág. 427)

Baccharido-Festucetum gracillimae ídem auctores 1985 Rel. 60

Lectotypus: TBPA, relevé 87 (pág. 427)

Bolaco-Festucetum gracillimae ídem auctores 1985 Rel. 61

Lectotypus: TBPA, relevé 320 (pág. 427)

XIX.- Festucetea thermari Freiberg 1985

Pastizales subandinos sud chilenos y argentinos

Festucetalia thermari Freiberg 1985

Perezio-Festucetum Freiberg 1985

XX.- Hippuretea vulgaris Roig, Anchorena, Dollenz, Faggi et Méndez 1985

Vegetación acuática semiflotante en aguas frías de escurrimiento lento.

Hippuretalia vulgaris Roig, Anchorena, Dollenz,

Faggi et Méndez 1985 (T)

Hippuridion vulgaris ídem auctores 1985 (T)

Hippuridetum vulgaris ídem auctores 1985 Rel. 62

(Fig. 11b)

Lectotypus: TBPA, relevé 397 (pág. 447) 
XXI. Honkenyo-Elymetea Tüxen 1966

Vegetación de dunas del litoral marino.

Honkenyo-Elymion Fernández Galiano em.

Géhu, In: Géhu 1975

Phacelio-Leymetum arenari Reška s/f

XXII.- Hordeetea lechleri Roig, Anchorena, Dollenz, Faggi et Méndez 1985

Vegetación de pantanos del sur patagónico.

Hordeetalia lechleri ídem auctores 1985 (T)

Hordion lechleri ídem auctores 1985 (T)

Hordeetum lechleri ídem auctores 1985 Rel. 63

Lectotypus: TBPA, relevé 274 (pág. 446)

Artemisietum magellanici ídem auctores 1985 Rel. 64

Lectotypus: TBPA, relevé 268 (pág. 446)

Galio-Hydrocotyletum chamaemori ídem auctores

1985 Rel. 65

Lectotypus: TBPA, relevé 117 (pág. 446)

XXIII.- Hordeetea pubiflori Roig, Anchorena, Dollenz, Faggi et Méndez 1985

Pastizales de inundación periódica en el sur patagónico.

Hordeetalia pubiflori Roig, Anchorena, Dollenz,

Faggi et Méndez 1985 (T)

Carici-Festucion pallescentis ídem auctores 1985 (T)

Junco-Festucetum pallescentis ídem auctores 1985 (T) Rel. 66

Lectotypus: TBPA, relevé 470 (pág. 437)

Azorello-Festucetum pallescentis ídem auctores 1985 Rel. 67

Lectotypus: TBPA, relevé 402 (pág. 437)

Poo-Hordion pubiflori Roig, Anchorena,

Dollenz, Faggi et Méndez 1985, em.

Deschampsio-Hordeetum pubiflori ídem auctores 1985 (T) Rel. 68

Lectotypus: : TBPA, relevé 400 (pág. 437)

Poo-Trifolietum repentis ídem auctores 1985 Rel. 69

Lectotypus: TBPA, relevé 290 (pág. 437)

Pration repentis Méndez et Ambrosetti 1985

Nostoco-Limoselletum australis Méndez et

Ambrosetti 1985 Rel. 70

Lectotypus: TBPA, relevé 122 (pág. 682)

Junco Caricetum macrosolenis Méndez et Ambrosetti 1985 Rel. 71

Lectotypus: TBPA, relevé 123 (pág. 682)

Rumici-Deschampsietum Méndez et Ambrosetti 1985

Rel. 72

Lectotypus: TBPA 121 (pág. 682)

XXIV.- Hordeetea santacrucensis Faggi 1985b

Vegetación de márgenes o fondos secos de lagunas temporarias del sur patagónico.
Plagiobothryo-Acaenetalia platyacanthae Faggi 1985b, em.

Plagiobothryo-Acaenion platyacanthae Faggi 1985b, em.

Hordeo-Acaenetum platyacanthae Faggi 1985, em. Rel. 73

Lectotypus: TBPA, relevé 458 (pág.622)

Hordeo-Acaenetum platyacanthae scutellarietosum

Faggi 1985b, em. Rel. 74

Lectotypus: TBPA, relevé 178 (pág 622)

Hordeo-Acaenetum platyacanthae poetosum Faggi $1985 \mathrm{~b}$

Lectotypus: TBPA, relevé 176

Poetum atropidiformis Faggi 1985b Rel. 75

Lectotypus: TBPA, relevé 39 (pág 622)

Adesmio-Juncetum arctici Faggi 1985b, em. Rel. 76

Lectotypus: TBPA, relevé 155 (pág 622)

Deschampsio-Alopecuretum aequalis Faggi 1985b, em. Rel. 77

Lectotypus: TBPA, relevé 20

Scirpo-Boopidetalia australis Faggi 1985b, em.

Scirpo-Boopidion australis Faggi 1985b, em.

Scirpo-Boopidetum australis Faggi 1985b, em. Rel. 78

Lectotypus: TBPA, relevé 56

Puccinellietum magellanicae Faggi 1985b Rel. 79

Lectotypus: TBPA, relevé 68

Polygono-Chenopodietum antarctici Faggi 1985b, em. Lectotypus: TBPA, relevé 20

\section{XXV.- Lantano camarae-Chusqueetea} ramosissimae Oriol de Bolós, Cervi et Hatschbach 1991

Comunidades de bambúes trepadores características de la pluvisilvas atlánticas paranenses.

Lantano camarae-Chusqueetalia ramosissimae idem auctores 1991

Tremo micranthae-Guaduion trinii idem auctores 1991

Misiones (Argentina) una comunidad de Solanum granuloso-leprosum, Trema micrantha, Guadua trinii, Urera baccifera, Cecropia adenopus, etc. que debe pertenecer a esta alianza (Oriol de Bolós et al., 1991).

XXVI.- Larreetea divaricato-cuneifoliae Roig 1989

Comunidades de arbustos perennes y resinosos de la provincia del Monte.

Larreetalia divaricato-cuneifoliae Roig 1989

Larreion divaricato-cuneifoliae Roig 1989

Larreo-Artemisietum mendozanae Roig 1989 Rel. 80

Lectotypus: relevé 4 (pág. 211)

Larreo-Artemisietum mendozanae baccharidetosum Roig 1989 


\section{E. Martínez Carretero et al. - Prodromus Sinsistemático y Estudios Fitosociológicos}

Lectotypus: relevé 18 (pág. 211)

Larreetum cuneifoliae Roig 1989 Rel. 81 (Fig. 10d)

Lectotypus: relevé 2 (pág. 213)

Larreetum cuneifoliae Zuccagnietosum Roig 1989

Typus: relevé17 (pág. 213)

Lareetum cuneifoliae Acacietosum Roig 1989

Typus: relevé 20 (pág. 213)

Chuquirago-Stipetum hypsophillae Martínez

Carretero 2005 Rel. 82

Typus: relevé 137

Larreo divaricatae-Lycion chanari Roig et Martínez Carretero 1998

Larreo-Lycietum vergarae Roig et Martínez Carretero 1998 Rel. 83

Larreo-Aphyllochladetum sanmartiniani Roig et Martínez Carretero 1998 Rel. 84

XXVII.- Lemnetea Oriol de Bolós et Masclans 1955 Comunidades de pleustófitos en aguas dulces tranquilas.

Lemnetalia Oriol de Bolós 1955

[ver sintaxones sin rango: Lemna minuta-Salvinia minima Gesellsch. Wolffiella oblonga-Lemma gibba ass. (Territorio IV)]

XXVIII.- Molinio-Arrhenatherea Tüxen 1937

Praderas de pastos tiernos en suelo rico en nutrientes.

Triseto-Poetalia pratensis Roig, Anchorena,

Dollenz, Faggi et Méndez 1985

Triseto-Poion pratensis Roig, Anchorena, Dollenz, Faggi et Méndez 1985

Triseto-Poetum pratensis Roig, Anchorena, Dollenz, Faggi et Méndez 1985 (T) Rel. 85

Lectotypus: TBPA, relevé 312 (pág. 443)

Trifolio-Leucanthemetum idem auctores 1985 Rel. 86 Lectotypus: TBPA, relevé 101 (pág. 443)

Holco-Acaenetum ovalifoliae idem auctores 1985 Rel. 87

Lectotypus: TBPA, relevé 106 (pág. 443)

Plantaginetalia majoris Tüxen 1950

Lolio perennis-Plantaginion majoris Sissingh 1969

Lolio perennis-Plantaginetum majoris Begert 1930

(?) em. Sissingh 1969

Polypogonetum elongati Méndez 1986, em. nom. inval.(art.3,d)

Trifolio fragiferi-Cynodontium dactylis Braun Blanquet et Oriol de Bolós 1958

Trifolio repentis-Cynodontetum dactylis Conticello, Bustamante et Cerazo 2008, nom. inval.

XXIX.- Mulino-Junellietea scopariae Roig 1989

Vegetación de arbustos perennifolios y pastizales duros del Cardonal (Pre-puna y Payunia).

Mulino-Junellietalia scopariae Roig 1989

Mulino-Junellion scopariae Roig 1989

Colliguajetum integerrimae Roig 1989 Rel. 88

Lectotypus: relevé 8 (pág.209)

Stipion tenuissimae Roig 1989

Stipetum tenuissimae Roig 1998 (T) Rel. 89

Lectotypus: relevé 9 (pág.205)

Saturejo-Adesmion uspallatensis Roig 1989

Mulinum-Nassauvietum axillaris Roig 1989 Rel. 90

Lectotypus: relevé 7 (pág.207)

Mulino-Adesmietum uspallatensis Rel. 91

Lectotypus: relevé 17 (además 11-16) (pág. 207)

Artemisietum echegarayi Roig et Martínez Carretero 1989 Rel. 92

Lectotypus: relevé 18 (pág. 141)

Nassauvio-Adesmietum horridae Martínez Carretero 2000 Rel. 93

Lectotypus: relevé 117 (pág. 207)

Grindelio-Stipetalia chrysophyllae Martínez

Carretero 2005

Matorrales y pastizales de Payunia

Stipo Junellion spathulatae glaucae Martínez Carretero 2005

Fabiano-Stipetum speciosae Martínez Carretero 2005 Rel. 94

Adesmio-Prosopidastretum Martínez Carretero 2005 Rel. 95

Neospartetum aphylli Martínez Carretero 2005 Rel. 96 (Fig. 10h)

Retanillo-Colliguajetum integerrimae Martínez Carretero 2005 Rel. 97

Stillingio-Mulinetum spinosi Martínez Carretero 2005 Rel. 98

Adesmietum pinifoliae Martínez Carretero 2005 Rel. 99

Stipetum chrysophyllae crispulae Martínez Carretero 2005 Rel. 100 (Fig. 10i)

Stipetum chrysophyllae crispulae ephedretosum Martínez Carretero 2005 Rel. 101

Stipetum speciosae mediae Martínez Carretero 2005 Rel. 102

Stipion speciosae Martínez Carretero 2005

Poetum durifoliae Martínez Carretero 2005 Rel. 103

XXX.- Myrteolo-Sphagnetea Oberdorfer 1960

Vegetación de turberas minerotróficas y ombrotróficas del sur magallánico.

Myrteolo-Sphagnetalia Oberdorfer 1960 (T)

Donation fascicularis Roig, Dollenz et Méndez $1985(\mathrm{~T})$ 
Donatietum fascicularis ídem auctores 1985 Rel. 104

Lectotypus: TBPA, relevé 118 (pág. 483)

Schoeno-Nothofagetum antarcticae ídem auctores 1985 Rel. 105

Lectotypus: TBPA, relevé 30 (pág. 483)

Sphagnion magellanici Roig, Dollenz et Méndez 1985

Donatio-Sphagnetum magellanici ídem auctores 1985 Rel. 106

Lectotypus: TBPA, relevé 77 (pág. 483)

Carico-Sphagnetum magellanici idem auctores 1985

Rel. 107

Lectotypus: TBPA, relevé 74 (pág. 483)

Marsippospermetum grandiflori $i$ dem auctores 1985 Rel. 108

Lectotypus: TBPA, relevé 118 (pág. 483)

Bolaco-Phyllachnetalia uliginosae Roig, Dollenz et Méndez 1985, em.

Bolaco-Phyllachnion uliginosae ídem auctores 1985 (T) em.

Unidades descriptas para el sur de Chile, pueden aparecer en Argentina.

Bolaco-Phyllachnetum uliginosi ídem auctores 1985

Rel. 109

Lectotypus: TBPA, relevé 68 (pág. 494)

Cryptochiletum grandiflori ídem auctores 1985 Rel. 110

Lectotypus: TBPA, relevé 14 (pág. 494)

Azorello-Phyllachnetum uliginosi ídem auctores 1985

Rel. 111

Lectotypus: TBPA, relevé 86 (pág.494)

Astelio-Phyllachnetum uliginosi ídem auctores 1985

Rel. 112

Lectotypus: TBPA, relevé 92 (pág. 494)

Pilgerodendro-Dacrydietum fonckii ídem auctores 1985, nom. mut. prop.

Basiónimo Lomatio-Dacridietum fonckii idem auctores 1985, inval., art.3 d. Rel. 113

Typus: TBPA, relevé 111 (pág.494)

\section{XXXI.- Nothofagetea pumilionis-antarcticae}

Oberdorfer 1960

Bosques caducifolios subantárcticos.

Nothofagetalia pumilionis-dombeyi Oberdorfer 1960 em. Eskuche 1968

Nothofagion pumilionis Oberdorfer $1960 \mathrm{em}$. Eskuche1999

Sin. Lagnophoro-Nothofagion pumilionis

Luebert, Gajardo et Estay 2003

Nothofagenion pumilionis Eskuche 2002

Macrachenio-Nothofagetum pumilionis Eskuche 1973b

Carici-Araucarietum Oberdorfer ex Finckh 1996
Nothofago pumilionis-Araucarietum araucanae Eskuche 2002 Rel. 114

Anemono-Nothofagetum pumilionis Oberdorfer 1960 ex Hildebrand-Vogel et al. 1990

Senecioni linariifolii-Nothofagetum pumilionis Eskuche 2002

Senecioni acanthifolii-Nothofagetum pumilionis Villagrán val. Eskuche 2002

Chiliotricho rosmarinifolii-Nothofagetum pumilionis Eskuche 2002 Rel. 115

Berberido ilicifoliae-Nothofagetum pumilionis Hildebrand-Vogel et al. 1990

Nothofagion dombeyi-obliqua Conticello et al. 1996, inval. art. 5; Eskuche 1999

Nothofagetum dombeyi-alpinae Conticello et al. 1996

Dioscoreo-Nothofagetum obliquae Eskuche 1973b

Austrocedro-Nothofagion dombeyi Eskuche 1968

Gavileo-Austrocedretum Eskuche 1968

Austrocedro-Nothofagetum Eskuche 1968

Violo magellanicae-Nothofagetalia pumilionis

Roig et al. 1985a; Hildebrand-Vogel, Godoy et Vogel 1990

Sin. Nothofagetalia pumilionis antarcticae Roig, Anchorena, Dollenz, Faggi et Méndez 1985

Violo magellanicae-Nothofagion pumilionis

Roig, Anchorena, Dollenz, Faggi et Méndez 1985

Violo magellanicae-Nothofagenion pumilionis (Roig et al. 1985a) Eskuche 2002

Pernettyo mucronatae-Nothofagetum pumilionis Eskuche 2002 (Fig. 10g)

Violo magellanicae-Nothofagetum pumilionis (Roig et al. 1985a) Eskuche 2002

Mayteno-Nothofagetum pumilionis Roig et al. 1985a

Elymo-Nothofagion antarcticae Roig, Anchorena, Dollenz, Faggi et Méndez, 1985, nom. mut. Basiónimo Agropyro-Nothofagion antarcticae ídem auctores 1985

Triseto-Nothofagetum antarcticae ídem auctores 1985 (T) Rel. 116

Lectotypus: TBPA, relevé 248 (pág.364)

Festuco-Nothofagetum antarcticae $i$ dem auctores 1985 Rel. 117

Lectotypus: TBPA, relevé 346 (pág. 364)

Escallonio virgatae-Nothofagion antarcticae

Roig, Anchorena, Dollenz, Faggi et Méndez 1985

Escallonio-Nothofagetum antarcticae ídem auctores 1985 (T) Rel. 118

Lectotypus: TBPA, relevé 110 (pág. 364)

Gunnero-Nothofagetum antarcticae ídem auctores 1985 Rel. 119 


\section{E. Martínez Carretero et al. - Prodromus Sinsistemático y Estudios Fitosociológicos}

Lectotypus: TBPA, relevé 127 (pág. 364)

Violo magellanicae-Acaenion pinnatifidae

Roig, Anchorena, Dollenz, Faggi et Méndez 1985

Violo-Polygaletum salasianae ídem auctores 1985 (T)

Rel. 120

Lectoypus: TBPA, relevé 115 (pág. 392)

Violo-Senecietum bracteolati ídem auctores 1985 Rel. 121

Lectotypus: TBPA, relevé 121 (pág. 392)

Berberido darwinii-Nothofagetalia antarctica

Eskuche 1969; Eskuche 1973b

Baccharido rosmarinifoliae-Discarion articulatae Eskuche 1969

Discarietum articulatae Eskuche 1969

Discario articulatae-Berberidetosum heterophyllae Eskuche 1969

Nothofago antarcticae-Berberidion Eskuche 1969

Ribesi-Nothofagion Eskuche 1969

Chacayo-Nothofagetum antarcticae Eskuche 1969, prov.

Senecioni-Nothofagetum antarcticae Eskuche 1969 prov., em.

Ribesi-Nothofagetum antarcticae Eskuche 1969

Ribesi-Nothofagetosum antarcticae Eskuche 1969

Ribes- Berberidetum parodii Eskuche 1969

Maytenio-Nothofagenion antarcticae Eskuche 1969

Lomatio hirsutae- Nothofagetum antarcticae Eskuche 1969

Lomatio hirsutae-Nothofagetum antarcticae Eskuche 1969

Lomatio hirsutae-Nothofagetum Antarcticadiostetosum junceae Eskuche 1969

Mutisio-Berberidetum darwinii Eskuche1969

Ribesi-Berberidetum parodii Eskuche 1969

Escallonietum virgatae Eskuche 1969, prov.

Berberidetum heterophyllae Eskuche 1969, prov.

Festuco scabriusculae-Araucarienion araucanae Gandullo 2003

Festuco scabriusculae-Araucarietum arancanae typicum Gandullo 2003 Rel. 122

Adesmio-Araucarietum Roig et Roig 1994 nom. inval. (Fig. 10f)

Adenocaulo-Nothofagetalia pumilionis Oberdorfer 1960; emend. Hildebrand-Vogel et al. 1990

Vicio-Nothofagenion pumilionis HildebrandVogel, Goday et Vogel 1990

Anemono antucensis-Nothofagetum pumilionis Oberdorfer 1960
Carici trichodes-Araucarietum araucanae Oberdorfer ex Finckh 1996

Drimydo-Nothofagion pumilionis Pollmann et Hildebrand 2005

Valeriano lapathifoliae-Nothofagetum pumilionis Flores-Toro et Hildebrand-Vogel 2006

Valeriano lapathifoliae-Nothofagetum pumilionis dombeyetosum Flores-Toro et Hildebrand-Vogel 2006

Valeriano lapathifoliae-Nothofagetum pumilionis gunneretosum magellanicae Flores-Toro, HildebrandVogel 2006

Drimydos andinae-Nothofagetum pumilionis Hildebrand-Vogel et al. 1990; Flores-Toro et HildebrandVogel 2006

Senecioni acanthifoli-Nothofagion pumilionis Pollmann et Hildebrand-Vogel 2005

Senecioni acanthifolii-Nothofagetum pumilionis Flores-Toro et Hildebrand-Vogel 2006

Senecioni acanthifolii-Nothofagetum pumilionis Typicum Flores-Toro et Hildebrand-Vogel 2006

Senecioni acanthifolii-Nothofagetum pumilionis gunneretosum magellanicae Flores-Toro et Hildebrand-Vogel 2006

Quinchamalio-Nothofagion pumilionis Pollmann et Hildebrand 2005

Festuco scabriusculae-Nothofagetum pumilionis Finckh 1996

Chiliotrichio diffusi-Nothofagion pumilionis Pollmann et Hildebrand 2005

Poo alopecuri-Nothofagetum pumilionis Hildebrand -Vogel 2002

Empetro rubri-Nothofagetum pumilionis Hildebrand -Vogel 2002

Austrocedro-Nothofagion dombeyi Eskuche 1968, 1999

Austrocedro-Nothofagetum dombeyi Eskuche 1968

Gavileo-Austocedretum Eskuche 1968

XXXII.- Notholaenetea nivea Gutte 1986

Comunidades saxícolas altoandinas de Perú y Bolivia, seguramente en Argentina.

XXXIII.- Panicetea racemosi Eskuche 1973a

Vegetación de dunas del litoral marítimo atlántico bonaerense.

Panicetalia racemosi Eskuche 1973a

Panicion racemosi Eskuche 1973a

Calycero-Panicetum racemosi (Cabrera 1941) em. Eskuche 1973a (Fig. 11h) 
Baccharidi-Androtrichion Eskuche 1973a

Vegetación de dunas fijas de arbustos enanos del litoral marítimo bonaerense.

Hyalidi-Baccharidetum genistifoliae Eskuche 1973a, prov

Androtricho-Baccharidetum genistifoliae (Cabrera 1941) em. Eskuche 1973a

Androtricho-Baccharidetum gnaphalioidis Eskuche 1973a, prov.

Androtricho-Baccharidetum leucopappae Eskuche 1973a, prov.

\section{XXXIV.- Panico urvilleani-Sporoboletea rigentis}

(prov. Eskuche 1973a) Eskuche 1992a, b

Comunidades vegetales de dunas móviles y fijas de la costa atlántica y en el interior en territorios templado a subtropical, árido a subárido.

Sporoboletalia rigentis Eskuche 1992a

Sporobolo rigentis-Panicion urvilleani (Cabrera 1941) em. Eskuche 1973a

Spartinetum ciliatae Hueck 1955

Elyonuro-Panicetum racemosi Martínez Crovetto 1962

Panico urvilleani-Sporoboletum rigentis (Cabrera 1941) em. Eskuche 1973a, 1992b

Senecioni quequensis-Panicetum urvilleanii Eskuche 1973a

Paspalo-Phyloxeretum portulacoidis Hueck 1950 em. Eskuche1973a

Phyloxero-Panicetum racemosi Eskuche 1973a

Calycero-Panicetum racemosi (Cabrera 1941) em. Eskuche 1973a

Calycero-Sporoboletum rigentis Martínez Carretero 2004 Rel. 123

Relevé typus: 152

Hyalietum argenteae latisquamatae Martínez Carretero $2004 \underline{\text { Rel. }} 124$

Releve typus: 171

Acicarpho-Alternantheretum maritimae Eskuche 1973a

Sporoboli baccharidion Eskuche 1973a, prov.

Sporoboli-Baccharidetum divaricatae Eskuche 1973a Rel. 125

Sporoboli-Psiletum spartioidis Eskuche 1973a Rel. 126

Sporobolion rigentis-atacamensis Ruthsatz 1977, prov.

Sporobolus rigens-atacamensis Ruthsatz 1977, prov.

Panico urvilleani-Brometalia brevis Eskuche 1992a

Panico urvilleani-Elyonuretum mutici Eskuche 1992a
Adesmio muricatae-Elyonuretum Eskuche 1992a Stipetum eriostachyae-tenuissimae Eskuche 1992a

Hypochoerio pampasicae-Panicion urvilleani Eskuche 1992a

Elyonuro mutici-Stipetum poeppigianae Eskuche 1992a

Bothriochloo saccharoidis-Panicetum urvilleani Eskuche 1992a

XXXV.- Pellaeetea tenuifoliae (alia, ion) Martínez Carretero 2005, prov.

Vegetación saxícola de pórfidos de la Payunia.

Pellaeetum tenuifoliae Martínez Carretero 2005, prov.

XXXVI.- Phragmito-Magnocaricetea Klika in

Klika et Novak 1941

Carrizales y totorales.

Phragmitetalia australis W. Koch 1926 em. Pignatti 1953

Phragmition australis Koch 1926 nom. mut.

Typho angustifoliae-Phragmitetum australis (Tüxen et Preissing 1942), Rivas Martínez et al. 1991

Typhetum Méndez 1984, inval. (art.10)

Scirpetum olneyi Méndez 1984

Lectoypus: relevé 107

Eleocharidetum albibracteatae Méndez 1984, em.

Lectotypus: relevé 77

Cyperetum eragrostis Méndez 1984

Lectotypus: relevé 5

Baccharidetum spartioidis Conticello, Gandullo, Bustamante et Tartaglia 1998 em., nom. inval. (?)

Psiletum spartioideae Conticello, Gandullo, Bustamante et Tartaglia 1997, $1998 \underline{\text { Rel. }} 127$

Holotypus: relevé 3

XXXVII.- Piptochaetio-Stipetea neesianae Eskuche 1992a

Pajonales y pastizales mesófilos de clima templado perhúmedo o húmedo desde el $\mathrm{S}$ de la provincia de Santa Fe hasta el E y S de la provincia de Buenos Aires.

Bothriochloo lagurioidis-Stipetalia neesianae

Eskuche 1992a

Stipion papposae Eskuche 1992a

Piptochaetio bicoloris-Stipetum charruanae Eskuche 1992a Rel. 128

Piptochaetium bicoloris-Stipetum clarazii Eskuche 1992a

Stipetum neesianae-hyalinae Eskuche 1992a (Fig. 11i) Melico eremophilae-Piptochaetion medii Eskuche 1992a, prov. 


\section{E. Martínez Carretero et al. - Prodromus Sinsistemático y Estudios Fitosociológicos}

Melico eremophilae-Piptochaetium medii Eskuche 1992a

Stipetalia bertrandii Eskuche 1992a

Stipion bertrandii Eskuche 1992a

Turnero pinnatifidae-Stipetum bertrandii Eskuche 1992a

\section{XXXVIII.- Pistio stratiotes-Eichhornietea} crassipedis Oriol de Bolós, A. Cervi et G.Hatschbach 1991

Comunidades de cormófios flotantes en aguas dulces.

Pistio stratiotes-Eichhornietalia crassipedis idem auctores 1991

Pistio stratiotes-Eichhornion crassipedis idem auctores 1991

Pistio stratiotes-Eichornietum crassipedis idem auctores 1991

Eskuche (1984:189) indica del norte de la República Argentina comunidades con Pistia stratiotes

Eichhornia azurea, E. crassipes y Reussia rotundifolia, evidentemente pertenecientes al Pistio-Eichhornion

(Oriol de Bolós et al., 1991).

XXXIX.- Plantagini rigidae-Distichietea

muscoidis Rivas-Martínez et Tovar 1982

Vegetación de turberas andinas, bofedales.

Calamagrostio jamesonii-Distichietalia muscoidis Rivas-Martínez et Tovar1982

Calamagrostio jamesonii-Distichion muscoidis Rivas-Martínez et Tovar 1982

Calamagrostio jamesoni-Distichietum muscoidis Rivas-Martínez et Tovar 1982

Scirpo deserticolae-Distichietum muscoidis Seibert et Menhofer 1991, 1992

Oxychloetum andinae Seibert et Menhofer 1991-1992 (Fig. 10e)

Wernerion pygmaeae Ruthsatz 1977 prov. Hypsela oligophylla ass. Ruthsatz 1977 prov.

Alchemilla pinnata-Eleocharis albibracteata ass. Ruthsatz 1977, prov.

Puccinellio oresigenae-Oxychloetum andinae Navarro 1993

Cassio-Trichocereion Ruthsatz 1977 prov.

\section{XL.- Polypodio squamulosi-Tillandsietea}

usneoidis Oriol de Bolós,

Cervi et Gert Hatschbach 1991

Comunidades de epífitas y comófitos neotropicales.

Tillandsietalia strictae idem auctores 1991

Tillandsion strictae ídem auctores 1991
Polypodio squamulosi-Tillandsietum strictae ídem auctores 1991

Es la principal vegetación epifítica de las selvas atlánticoparanenses.

Según la distribución geográfica de las principales especies, parece que la clase se extiende desde el sur de los EEUU hasta el norte argentino (Oriol de Bolós et al. 1991).

XLI.- Podocarpo parlatorei-Tipuanetea tipi Rivas-Martínez et Navarro 2002, In: Navarro et Maldonado 2002

Mesobosques sempervirentes, estacionales y semideciduos, termo y mesotropicales en áreas xéricas.

Tipuano tipus-Podocarpetalia parlatorei ídem auctores 2002

Juglandi australis-Phoebion porphyrae ídem auctores 2002

Schinetum myrtifolio-gracilipedis Entrocassi 2015

Juglandi australis-Blepharocalycetum salicifolii Entrocassi 2015

Zanthoxylo cocoi-Blepharocalycetum salicifolii Entrocassi 2015

Myrciantho callicomae-Podocarpion parlatorei Rivas-Martínez et Navarro 2002

Pruno tucumanensis-Podocarpetum parlatorei Rivas-Martínez et Navarro 2002

Myrciantho pseudomatoi-Blepharocalycetum salicifolii Entrocassi 2015

Cinamomo porphyrium-Blepharocalycetum salicifolii Entrocassi 2015

Xylosmo pubescentis-Blepharocalycetum salicifolii Entrocassi 2015

Parapitadenio excelsae-Tipuanion tipi RivasMartínez et Navarro 2002

Enterolobio contortisilici-Anadenantherum cebilis Entrocassi 2015

Jacarando mimosifoliae-Vassobietum breviflorae Entrocassi 2015

Erythrino falcatae-Tipuanetum tipi Entrocassi 2015

XLII.- Quinchamalio-Pernettyetea pumilae Freiberg 1985

Matorrales enanos subantárcticos.

Quinchamalio-Pernettyetalia Freiberg 1985

Pernettyion pumilae Freiberg 1985

Erigoni-Pernettyeum Freiberg 1985

XLIII.- Rostkovietea Roig, Dollenz et Méndez 1985 Turberas temporalmente cubiertas por aguas frías, de origen pluvial o nival. 
Rostkovietalia ídem auctores 1985

Rostkovion ídem auctores 1985

Rostkovietum magellanici idem auctores 1985 Rel. 129

Lectotypus: TBPA, relevé 50 (pág. 499)

XLIV.- Ruderali-Manihotetea utilissima Léonard in Taton 1949 em. nom. Oriol de Bolós 1988

Comunidades ruderales pantropicales, de trepadoras herbáceas, que cubren vallados. Observadas en Chile y Argentina por Oberdorfer (1960:57-59) (Oriol de Bolós et al., 1991).

Ipomoeetalia purpureae (Oberdorfer) Oriol de Bolós 1988

XLV.- Salicetea humboldtianae (Oberdorfer 1960 prov.) Eskuche 2004

Comunidades de la sucesión primaria sobre sedimentos aluviales recientes que bordean los ríos en el $\mathrm{NE}$ de Argentina.

Tessario integrifoliae-Salicetalia humboldtianae Eskuche 2004

Tessario absinthioidis-Salicion humboldtianae

Eskuche 2004

Tessario absinthioidis-Baccharidetum marginalis Oberdorfer 1960 prov.

Tessario integrifoliae-Salicion humboldtianae

Eskuche 2004

Acacio macracanthae-Tessarietum integrifoliae

Eskuche 2004

Tessario integrifoliae-Salicetum humboldtianae Eskuche 2004 Rel. 130

Holoypus. Rel. 2049

Cathormio-Ingion Eskuche 2004 (Clase y Orden aún a establecer)

Eugenio repandae-Cathormietum polyanthi Eskuche et Fontana 1996a

Bergeronio-Cathormietum polyanthi Eskuche 2004

Rel. 131

Holotypus: Rel. 2892

Cecropio pachystachae-Crotonetum urucuranae Eskuche et Fontana 1996a

Cisso palmatae-Solanetum amygdalifolii Eskuche 2004 Rel. 132

Holotypus: Rel. 2790

XLVI.- Salici humboldtiani-Prosopietea albae Rivas-Martínez et Navarro 2002, In: Navarro et Maldonado 2002

Micro-mesobosques freatófitos y ribereños, en bioclima infratropical, termotropical y mesotropical inferior.

Salici humboldtiani-Prosopietalia albae RivasMartínez et Navarro 2002
Prosopion albae Rivas-Martínez et Navarro 2002 Salici humboldtianae-Acacietum aromae Entrocassi 2015

XLVII.- Salvinio minimae-Lemnetea valdivianae Eskuche 2004

Vegetación acuática de las vegas del río Paraná y sus afluentes, con plantas flotantes o arraigadas en el fondo o en riberas de los cuerpos de agua.

Spirodelo intermediae-Lemnetalia valdivianae idem auctores 2004

Azollo-Lemnion valdivianae idem auctores 2004

Lemno minutae-Salvinietum minimae idem auctores 2004 Rel. 133

Holotypus: Rel. 2558

Lemno valdivianae-Wolffielletum gladiatae Landolt 1981 (1999)

Pistio-Eichhornietalia crassipedis Eskuche 2004

Limnobio-Pistion prov.

Limnobio-Pistietum Eskuche 2004 Rel. 134

Holotypus: Rel.2498

Eichhornion crassipedis-azureae Eskuche 2004

Reussio-Eichhornietum crassipedis Eskuche 2004

Myriophyllo-Eichhornietum azureae Eskuche 2004

Panicetalia grumosi-prionitidis Eskuche 2004

Panicion grumosi Eskuche et Fontana 1996b

Cupheo melvillae-Panicetum grumosi Eskuche et Fontana 1996b Rel. 135

Holotypus: relevé 5

Sisyrinchio-Axonopodetum compressi Eskuche et Fontana 1996b Rel. 136

Holotypus: relevé 3

Paspaletum exaltati-haumanii Eskuche et Fontana 1996b, prov.

Panico grumosi-Polygonetum acuminatae Eskuche et Fontana 1996b Rel. 36

Panicion prionitidis Eskuche 2004

Crotono tenuissimi-Panicetum prionitidis Eskuche et Fontana 2004 (in Eskuche 2004)

Rhabdocaulo-Panicetum prionitidis Eskuche 2004

Vernonio incanae-Panicetum prionitidis Eskuche 2004

XLVIII.- Sarcocornietea perennis (Faggi 1985b)

Martínez Carretero nom. mut. prop. 2001

(Basiónimo: Salicornietea ambigua Faggi 1985b)

Comunidades arbustivas halófilas.

Sarcocornietalia perennis Martínez Carretero 2001 statio nova

(Basiónimo: Salicornitalia ambigua Faggi 1985b)

Sarcocornion perennis Martínez Carretero 2001 


\section{E. Martínez Carretero et al. - Prodromus Sinsistemático y Estudios Fitosociológicos}

Puccinellio-Sarcocornietum Faggi 1985b, Martínez Carretero 2001 Rel. 137

Lectotypus TBPA 5

(Basiónimo: Puccinellio-Salicornietum ambiguae Faggi 1985b

Frankenio-Sarcocornietum (Faggi 1985b) Martínez Carretero 2001

(Basiónimo Frankenio Salicornietum ambiguae Faggi 1985

Suaedo-Sarcocornietum Faggi 1985b, Martínez Carretero 2001 Rel. 138 (Fig. 10c)

(Basiónimo Suaedo-Sarconietum ambiguae Faggi 1985b

Lectotypus TBPA 161

Distichletalia spicatae Martínez Carretero 2001

Distichlion spicatae Martínez Carretero 2001, 2004

Distichletum spicatae ídem auctor 2001 Rel. 139

Distichletum spicatae-Suaedo argentinetosum ídem auctor 2001, 2004

Distichletum spicatae-Frankenio juniperetosum idem auctor 2001,2004

Distichletum spicatae-Baccharido spartioidetosum ídem auctor 2001, 2004

Distichletum spicatae-Junceto arcticetosum ídem auctor 2001

Nithrophilo-Distichletum scopariae Gandullo 2004 Rel. 140

Typus Relevé: 2

Suaedetum neuquenensis Gandullo et Gastiazoro 2009 Lepidophylletalia (Faggi 1985b) Martínez Carretero nov. statio 2005

(Basiónimo Lepidophylletea cupressiformis Faggi 1985b)

Matorral de desiertos salinos de clima frío del sur patagónico y Tierra del Fuego.

Lepidophyllion cupressiformis Faggi 1985b

(Basiónimo: Lepidophylletea cupressiforme Faggi 1985b)

Puccinellio magellanicae-Lepidophylletum cupressiforme Faggi 1985b Rel. 141

Lectotypus: relevé 159 (pág. 613)

Puccinellio magellanicae-Lepidophylletum poetosum Faggi $1985 \mathrm{~b}$

Puccinellio magellanicae-Lepidophylletum sarcocornietosum (Faggi 1985b), Martínez Carretero 2005

(Basónimo: Puccinellio magellanicaeLepidophylleto Salicornietosum Faggi 1985b

Puccinellio magellanicae-Lepidophylletum Suaedetosum Faggi 1985b
Puccinellio mendocinae-Lepidophylletum Faggi 1985b Rel. 142

Lectotypus: relevé 2 (pág. 613)

Poo-Lepidophylletum Faggi 1985b Rel. 143

Lectotypus: relevé 9 (pág. 613)

Psiletalia spartioidae Conticello et al. 1998, prov.

Psilion spartioidae Conticello et al. 1998, prov

Psiletum spartioidae Conticello et al. 1999

XLIX.- Senecionetea bipontini Oberdorfer 1960

Vegetación de acarreos o pendientes de cascajo en alta montaña.

Senecionetalia bipontini Freiberg 1985

Nassauvion revolutae Freiberg 1985

Nassauvietum revolutae Freiberg 1985

L.- Senecioni-Proustietea cuneifoliae Roig 1989, em.

Vegetación riparia de ríos temporarios.

Senecioni-Proustietalia cuneifoliae Roig 1989

Senecioni-Proustion cuneifoliae Roig 1989

Senecioni-Proustietum cuneifoliae Roig 1989

LI.- Soncho-Bidentetea pilosi Hoff in Hoff et Brisse 1983

Vegetación nitrófila viaria andina o de áreas abiertas utilizadas por el hombre, como zonas abandonadas del pastoreo o incendiadas.

Bidentetalia pilosae Lebrun In: Mullenders 1949

Eleusinion indicae Léonard 1950

Eleusino tristachyae-Cynodontetum dactyli Eskuche et Iriart 1996 a, b Rel. 3

LII.- Stellarietea mediae (Braun-Blanquet 1931) Tüxen., Preising et Lohm 1950 (In: Tüxen 1950)

Vegetación con predominio de especies anuales, ruderales, adventicias, etc., en suelos ricos en nitrógeno.

Chenopodietalia albi (Sissingh In: Westhoff, Dijk \&

Passchier 1946) Tüxen et Lohmeyer (in Tüxen 1950)

Sisymbrion Méndez 1983, nom. inval.

Eragrostio-Diplachnetum uninerviae-procumbentis Méndez 1990 Rel. 144

Lectotypus: relevé 73

Kochietum scopariae Conticello, Bustamante, Gandullo et Tartaglia 1998 Rel. 145

Lectotypus, relevé 14

Malvo-Lamietum Méndez 1984, nom. inval., art. 10.

Sisymbrio-Rapistretum Méndez 1983, nom. inval., art.10

Panico-Setarion Méndez, nom. inval. art. 10

Echinochloetum crucis galli Méndez 1984, nom. inval., em. 
Erigerono-Cynaretum cardunculi Méndez $1990 \underline{\text { Rel. }}$ 146

Letcotypus: relevé 14

Setarietum verticillatae Méndez 1984 Rel. 147

Lectotypus: relevé 16

Pitraeo-Amaranthetum quitensis Méndez 1990

Rel. 148

Lecotypus: relevé 15

Portulaco-Chenopodietum albi Méndez 1990 Rel. 149

Lectotypus: relevé 57

Foeniculetalia Méndez 1984

Foeniculion Méndez 1984, nom. inval.

Baccharidetum spartioidis Conticello, Bustamante, Gandullo et Tartaglia 1998, nom.mut.

Foeniculetum vulgare Méndez 1984 Rel. 150

Lectotypus: relevé 58 (Tab. 1)

Imperatetum condensatae Méndez 1984 Rel. 151

Lectotypus: relevé 132 (Tab. 1)

Paspaletum dilatati Méndez 1984, nom. inval.

Wedelietalia glaucae Méndez 1983

Wedelion glaucae Méndez 1983

Baccharidetum salicifoliae Méndez 1983 Rel. 152

Lectotypus: relevé 164 (Tab. 1)

Cichorio-Erodietum cicutarii Méndez 1983 Rel. 153

Lectotypus: relevé 20 (Tab. 2)

Cynodetum dactyli Méndez 1983 Rel. 154

Lectotypus: relevé 83 (Tab. 1)

Lepidietum drabae Méndez 1983 Rel. 155

Lectotypus: relevé 141 (Tab. 1)

Lepidio-Erodietum cicutari Méndez 1983 Rel. 156

Lectotypus: relevé 10 (Tab. 2)

Solano-Chenopodietum albi Méndez 1983 Rel. 157

Lectotypus: relevé 29 (Tab. 2)

Wedelietum glaucae Méndez 1983a Rel. 158

Lectotypus: relevé 40 (Tab. 1)

Wedelio-Paspaletum Méndez 1983, nom. inval., art.10

Conyzo-Asterion Méndez 1983

Conyzo-Asteretum Méndez 1983, nom. inval., art.10

Lepidietum Méndez 1983, nom inval. art.10

Solano-Polygonetalia R. Tx. et Lohm. In: Tüxen

1950 em. J. Tx. 1966

Diplotaxion erucoidis Braun Blanquet 1931, In:

Braun-Blanquet, Gajewski, Wraber \& Walas 1936 em. Brullo \& Marceno 1980

Portulaco oleraceae-Diplotaxietum erucoidis Méndez 2003 Rel. 159

Holotypus: relevé 13

Lamio amplexicaulis-Urticion urentis Méndez 2015
Lamio amplexicaulis-Urticetum urentis Méndez 2015

Holotypus releve 12

LIII.- Stipo-Lycietea fusci Roig et Martínez Carretero 1998

Arbustos y pastos duros del sector austral de la Puna.

Stipo-Lycietalia fusci Roig et Martínez Carretero 1998

Chuquirago-Lycion fusci Roig et Martínez Carretero 1998

Artemisietum paramilloensis Martínez Carretero, Dalmasso, Márquez et Martinelli 2009 Rel. 160

Lectotypus: relevé 7

Baccharidetum incari fusci Roig et Martínez Carretero 1998 Rel. 161

Sintypus: relevé 21

Chuquirago-Lycietum fusci Roig et Martínez Carretero 1998 Rel. 162 (Fig. 10a)

Sintypus: relevé 1

Variente con Fabiana patagonica Martínez Carretero 1998

Chuquirago-Lycietum fusci atriplicetosum Roig et Martínez Carretero 1998

Jaravetum chrysophyllae Martínez Carretero, Dalmasso, Márquez et Martinelli 2009 Rel. 163

Lectotypus: relevé 39

Lycio-Ephedretum rupestri Martínez Carretero, Dalmasso, Márquez et Martinelli 2009 Rel. 164

Lectotypus: relevé 33

Senecionetum grindeliaefolii Roig et Martínez Carretero 1998 Rel. 165

Sintypus: relevé 26

Glandulario-Ipomopsion gossypiferae Roig et Martínez Carretero 1998

Glandulario-Phacelietum sinuatae Roig et Martínez Carretero 1998 Rel. 166

Sintypus: relevé 32

Astragalo-Ephedretum breanae Roig et Martínez Carretero 1998 Rel. 167

Sintypus: relevé 46

Ephedro-Stipion scirpeae Roig et Martínez Carretero 1998

Stipetum scirpeae Roig et Martínez Carretero 1998 Rel. 168 (Fig. 10b)

Sintypus: relevé 54

Stipo-Junellietum uniflorae Roig et Martínez Carretero 1998 Rel. 169

Sintypus: relevé 50

Melico-Salvion gilliesii Roig et Martínez Carretero 1998 


\section{E. Martínez Carretero et al. - Prodromus Sinsistemático y Estudios Fitosociológicos}

Aphanostelmetum candolleanii Roig et Martínez Carretero 1998 Rel. 170

Sintypus: relevé 60

Plazietum daphnoidis Roig et Martínez Carretero 1998

Rel. 171

Sintypus: relevé 66

Sisymbrietum arnottii Roig et Martínez Carretero 1998

Rel. 172

Sintypus: relevé 69

Gymnophytetum polycephali Roig et Martínez Carretero 1998 Rel. 173

Sintypus: relevé 98

LIV.- Suaedetea divaricatae Alonso et Conticello ex Martínez Carretero 2001

Vegetación de suelos salinos de regiones cálidas.

Stenodrepanum-Prosopietalia reptantis Martínez

Carretero 2001, 2004

Cortesio-Prosopietalia strombuliferae Martínez

Carretero 2001, 2004

Cortesio-Prosopion strombuliferae Martínez Carretero 2001

Lycio-Sporoboletum mendocinae Martínez Carretero 2001, nom. inval.

Atripleci roseae-Halophytetum ameghinoi Gandullo, Gastiazoro et Schmid 2010 Rel 174

Salicornetalia ambiguae Faggi 1985b

Salicornion ambiguae Faggi 1985b

Distichlo-Salicornietum ambiguae Conticello, Bustamante et Tartaglia 1997

LV.- Verrucarietea Roig, Dollenz et Méndez 1985, prov.

Asociaciones de líquenes en playas rocosas periódicamente cubiertas por el agua salada.

Verrucaria sp.- Hildebrandia lecanellieri ass. Roig, Dollenz et Méndez 1985

Caloplaca sublobulata ass. Roig, Dollenz et Méndez 1985

LVI.- Wintero-Nothofagetea Oberdorfer 1960

Bosques caducifolios húmedos a magallánico perennifolio.

Laurelietalia philippianae Oberdorfer 1960

Nothofago dombeyo-Eucryphion cordifolia Oberdorfer 1960

Nothofago-Dombeyo-Eucryphietum cordifoliae Oberdorfer 1960

Aextoxiconenion punctati Oberdorfer 1960

Lapagerio rosae-Aextoxiconetum punctati Oberdorfer 1960
Nothofago obliquae-Perseetum lingue Oberdorfer 1960

Dasiphyllo diacanthoidis-Nothofagetum alpinae Frank. et Fickh in Pollmann 2001

Berberido trigonae-Nothofagetalia dombeyi

Pollmann 2001

Myrceugenio-Nothofagion dombeyi (Eskuche 1999) Pollmann 2001

Myrceugenio-Nothofagetum dombeyi Eskuche 1999

Nothofagetum dombeyi-alpinae Conticello et al. 1996; Eskuche 1999

Nothofagetosum pumilionis Eskuche 1999

Dioscoreo-Nothofagetum obliquae Conticello et al. 1996; Eskuche 1973b, 1999

Austrocedro-Nothofagion dombeyi Eskuche 1968, 1999

Austrocedro-Nothofagetum dombeyi Eskuche 1968

Gavileo-Austrocedretum Eskuche 1968

Wintero-Nothofagetalia betuloidis (Roig, Dollenz et Méndez 1985) Pollmann 2001

Weinmannienion trichospermae Oberdorfer 1960

Laurelio philippianae-Weinmannietum trichospermae Oberdorfer 1960

Saxegotheo-Weinmannietum Villagrán 1980, prov.

Fitzroyetum cupressoides Oberdorfer 1960

Pilgerodendronetum uviferi Oberdorfer 1960

Nothofagion betuloidis (Oberdorfer 1960) Roig

et al. $1985 \mathrm{~b}$ ex Pollmann 2001

Nothofagetum betuloidis Oberdorfer 1960

Hebo-Pernettyetum mucronatae Roig et al. $1985 \mathrm{~b}$

Rel. 175

Lectotypus: TBPA, relev. 70

Embothryo-Nothofagion betuloidis Roig et al. $1985 \mathrm{~b}$

Embothrio-Nothofagetum betuloidis Roig et al. 1985b Rel. 176

Lectotypus: TBPA, relev. 60

Lomatio-Nothofagetum betuloidis Roig et al. 1985b Rel. 177

Lectotypus: TBPA, relev. 129 (pág. 470)

Baccharido-Nothofagetum betuloidis Roig et al. 1985b Rel. 178

Lectotypus: TBPA, relev. 27 (pág. 470)

Acaeno-Gunnerienion magellanicae Roig et al. $1985 \mathrm{~b}$

Acaeno-Gunneretum magellanicae Roig et al. 1985b Rel. 179

Lectotyus: TBPA, relev. 53 (pág. 470)

Ribo-Pernettyetum mucronatae Roig et al. $1985 \mathrm{~b}$ Rel. 180

Lectotypus: TBPA, relev. 65 (pág.470) 
Aristotelietalia chilensis Hildebrand-Vogel 1983

Matorrales secundarios con Aristotelia chilensis, posiblemente en el N de Chubut y sur de Río Negro.

Paludo-Myrceugenetalia Oberdorfer 1960

Myrceugenion exsuccae Oberdorfer 1960

Comunidades hidrófilas de arrayán en orillas de lagos y ríos de Argentina y Chile.

\section{LVII.- Xyrido carolinianae-Typhetea}

domingensis Oriol de Bolós, Cervi et Hatschbach 1991

Comunidades helofíticas neotropicales en esteros con herbáceas gigantes.

Equisetum gigantei-Typhetalia domingensis idem auctores 1991

Equiseto gigantei-Typhion domingensis $i$ dem auctores 1991

Equiseto gigantei-Typhietum domingensis idem auctores 1991

Eskuche (1984: 189) indica esteros con Typha domingensis, Cyperus giganteus, y Schoenoplectus californicus para el valle del Iguazú, que no sabemos si pueden incluirse en esta asociación o en otra afín (Oriol de Bolós et. al. 1991)

\section{LVIII. - Comunidades de epífitas del NE Argentino Drehwald 1995}

(Sintaxones de orden superior)

Comunidades de musgos, hepáticas y líquenes sobre troncos en descomposición del NE de Argentina

Sematophylletum riparioides Drehwald 1990

Fissidentetum oedilomae idem auctores 1990

Leptodontio-Schlotheimietum Drehwald 1995 Rel. 181

Holotypus: relevé 4 (Tab.10)

Epidendron paniculatum-Vriesia friburgensis idem auctores 1995 Rel. 182

Lectotypus: relevé 3 (Tab. 11)

Piliotrichello-Orthostichopsietum tenuis idem auctores 1995 Rel. 183

Lecotypus: relevé 1 (Tab. 12)

Piliotrichello-Neckeropsietum undulatae idem auctores 1995 Rel. 184

Holotypus: relevé 23 (Tab. 14)

Zygopetalo-Hymenodontetum idem auctores 1995

Rel. 185

Holotypus: relevé 1 (Tab. 17)

Porotrichetum lancifrontis idem auctores 1995 Rel. 186

Holotypus: relevé 6 (Tab. 18)

Hypopterigetum tamariscini idem auctores 1995 Rel. 187

Holotypus: relevé 2 (Tab. 19)

Uleastretum paraguensis idem auctores 1995 Rel. 188
Holotypus: relevé 10 (Tab. 23)

Callicostello-Vesicularietum vesicularis idem auctores 1995 Rel. 189

Holotypus: relevé 21 (Tab. 20)

Tillandisetum duratii-loliaceae idem auctores 1995

Rel. 190

Holotypus: relevé 9 (Tab. 21)

Erpodietum beccarii idem auctores 1995 Rel. 191

Holotypus: relevé 6 (Tab.22)

Microgrammo-Tillandsietum tricholepidis idem auctores 1995 Rel. 192

Holotypus: relevé 20 (Tab. 24)

Fissidentetum brevipedis idem auctores 1995 Rel. 193

Holotypus: relevé 4 (Tab. 25)

Stereophylletum radiculosi idem auctores 1995 Rel. 194

Holotypus: relvé 6 (Tab. 26)

Isopterygio-Chryso-hypnetum diminutivi idem auctores 1995 Rel. 195

Holotypus: relevé 1 (Tab. 27)

Orthotricho-Frullanietum bonariensis Drehwald et Reiner-Drehwald 1996

\section{Sintaxones probables en Argentina}

LIX.- Acacio feddeannae-Prosopidetea ferocis Rivas-Martínez et Navarro 2002, In: Navarro et Maldonado 2002

Pequeños bosques ricos en cactáceas de la prepuna del $\mathrm{S}$ de Bolivia y NO de Argentina.

Prosopidion ferocis Navarro et Maldonado 2002

LX.- Bidentetea tripartitae Tüxen, Lohmeyer et Preising In: Tüxen 1950 ex von Rochow 1951

De origen europeo, agrupa vegetación higro-nitrófila en sedimentos de cauces, acequias y vaguadas en general.

LXI.- Cladietea jamaisencis Knapp 1964 ex Borhidi 1996 -en: XVIII.- Hippuretea vulgaris Roig, Anchorena, Dollenz, Faggi et Méndez 1985-

Vegetación de Humedales del Chaco y el cerrado

Scirpo-Eleocharitetalia interstinctae Borhidi et Muñiz in Borhidi 1996

Typhion domingensis Del Risco in Borhidi 1996

Rhabdadenio ragonesei-Cyperetum gigantei Galán de Mera et Navarro 1992

LXII. Deuterocohnio longipetalae-Puyetea ferrugineae Rivas-Martínez \& Navarro 2002, In: Navarro et Maldonado 2002

Vegetación fisurícola de ambientes áridos desde el $\mathrm{C}$ de Bolivia al C de Argentina. 


\section{E. Martínez Carretero et al. - Prodromus Sinsistemático y Estudios Fitosociológicos}

Polypodio pycnocarpi-Puyetalia ferrugineae

Galán de Mera, Rosa et Cáceres 2002

Deuterocohnion strobilifero-longipetalae

Navarro et Maldonado 2002

LXIII.- Eichhornietea crassipedis Galán de Mera et Navarro 1992

Vegetación acuática pionera integrada por pleustoheliófitos.

Eichhornietalia crassipedis Galán de Mera et Navarro 1992

Eichhornion azurae Borhidi et Muñiz in Borhidi,

Muñiz et Del Risco 1979, em. Galán de Mera et Navarro 1992

Eichhornietum azurae Borhidi et Muñiz in Borhidi, Muñiz et Del Risco 1983

Eichhornion crassipedis Galán de Mera et Navarro 1992

Eichhornietum crassipedis Samek et Moncada 1971

LXIV.- Fabiano bryoidis-Stipetea frigidae RivasMartínez et Navarro 2002, In: Navarro et Maldonado 2002

Pajonales con pequeños arbustos y matorrales pulviniformies que se desarrollan sobre los $1400 \mathrm{~m}$, en áreas áridas, volcánicas, en la cordillera andina occidental de Bolivia, Chile y Argentina.

Fabiano bryoidis-Stipetalia frigidae Navarro et Maldonado 2002

Urbanio pappigerae-Stipion frigidae Navarro 1993

\section{LXV.- Galio aparines-Urticietea dioicae Passarge 1967 \\ Vegetación escionitrófila de origen europeo, en la semisombra de vaguadas, muros y bosques en suelos temporalmente frescos.}

LXVI.- Lemnetea minoris Tüxen 1953 ex Oriol de Bolós et Masclans 1955

Vegetación cosmopolita con especies con órganos asimiladores flotantes o suspendidos entre el fondo y la superficie.

Lemnetalia aequinoctialis Schwabe-Braun et Tüxen 1981

Azollo carolinianae-Salvinion auriculatae Borhidi et Muñiz in Borhidi, Muñiz et Del Risco 1979

Spirodelo polyrhizae-Salvinietum auriculatae Borhidi et Muñiz in Borhidi, Muñiz et Del Risco 1979
LXVII.- Lippio bolivianae-Dodonaetea viscosae Rivas-Martínez et Navarro 2002, In: Navarro et Maldonado 2002

Matorrales y arbustedas deciduos de los valles interandinos del S de Bolivia y N de Argentina.

Lippio bolivianae-Acalyphion lycioidis Navarro et Maldonado 2002

LXVIII.- Loxopterygio grisebachii-Schinopsietea haenkeanae Rivas-Martínez \& Navarro 2002 in Navarro \& Maldonado 2002

Vegetación xerofítica de los Andes centrales y meridionales de Bolivia y septentrionales de Argentina.

Loxopterygio grisebachii-Schinopsion haenkeanae Navarro \& Maldonado 2002

LXIX.- Nicotiano glutinosae-Ambrosietea arborescentis Galán de Mera et Cáceres 2002, In: Galán de Mera, Rosa et Cáceres 2002

Vegetación terofítica de talla elevada (1-2m) neotropical andina con irradiaciones en las áreas templadas de Argentina y Chile.

Nicotianetalia paniculato-glutinosae Galán de Mera et Cáceres 2002 in Galán de Mera, Rosa et Cáceres 2002

LXX.- Opuntietea sphaericae Galán de Mera et Orellana 1996

Matorral bajo microfilo de los desiertos andinos del $\mathrm{N}$ de Chile y N de Argentina.

Atriplicetalia imbricatae Luebert et Gajardo 2005

Ambrosio artemisioidis-Atriplicion imbricatae Luebert et Gajardo 2000

Sicyio baderoae-Urticion magellanicae Galán de Mera et Cáceres 2002 in Galán de Mera, Rosa et Cáceres 2002

LXXI.- Polylepidetea tarapacano-besseri RivasMartínez et Navarro 2002, In: Navarro et Maldonado 2002

Quiñuales de la Puna seca del S de Bolivia y $\mathrm{N}$ de Argentina

Polylepidion tomentello-tarapacanae Navarro, In: Navarro et Maldonado 2002

LXXII.- Polygono-Poetea annuae Rivas Martínez 1975, corr. Rivas Martínez, Báscones, Díaz, González et Loidi 1991

Comunidad de anuales nitrófilas en suelos compactados.

Polygono arenastri-Poetalia annuae Tüxen In: Géhu, Rich. et Tüxen 1972 corr. Rivas Martínez, Báscones, Diáz, González et Loidi 1991 
Polycarpion tetraphylli Rivas Martínez 1975

Bromo cathartici-Coronopodetum didymi Moglia 2006

LXXIII.- Ruprechtio triflorae-Aspidospermetea quebracho-blanconis Galán de Mera 2001, In: Navarro et Maldonado 2002

Bosque climácicos subhúmedos-húmedos del Chaco Boreal. Bosques xerofíticos climácicos del Chaco seco boreal (450-750 mm), del S de Bolivia, NE de Paraguay y $\mathrm{N}$ de Argentina.

Schinopsion quebracho-coloradonis Galán de Mera 2001

\section{SINTAXONES SIN RANGO}

Territorios florísticos

I.-Territorio Austral

(Provincias Subantártica, Patagónica y de la Payunia)

II.-Territorio Andino

(Prov. Andina, de la Puna y del Cardonal)

III.- Territorio Central

(Prov. Chaqueña, Pampeana, del Espinal y del Monte)

IV.- Territorio de las Selvas subtropicales

(Prov. Paranense y de las Yungas)

I.-Territorio Austral

(Provincias Subantártica, Patagónica y de la Payunia)

\section{1.- PROVINCIA SUBANTÁRTICA}

a- de bosques, rodales diversos, etc.

Discaria trinervis-Cortaderia rudiuscula De Marco et al. 1993

Drimys winteri-Myrceugenia exsuccae Villagrán 1980

Myrceugenella apiculata Villagrán 1980

Nothofagus antarctica-Berberis darwinii Seibert 1979

Nothofagus antarctica Dudley et Crow 1983

Nothofagus antarctica Collantes, Ontivero, Bianciotto 1990

Nothofagus antarctica-Carex canescens Roig 2000

Nothofagus antarctica-Chusquea culeou Seibert 1979

Nothofagus antarctica-Gunnera magellanica Roig 2000

Nothofagus antarctica-Hierochlöe redolens Roivainen 1954
Nothofagus antarctica-Marsippospermum grandiflorum Dollenz 1985-86

Nothofagus antarctica-Osmorhyza chilensis Anchorena 1985

Nothofagus betuloides-Drimys winteri Dudley et Crow 1983

Nothofagus betuloides-Drimys winteri Dollenz 198586

Nothofagus betuloides Collantes, Ontivero, Bianciotto 1990

Nothofagus betuloides-Lebetathus myrsinites Dollenz 1985-86

Nothofagus betuloides-Marsippospermum grandiflorum Dudley et Crow 1983

Nothofagus betuloides-Nothofagus pumilio Collantes, Ontivero, Bianciotto 1990

Nothofagus pumilio-Berberis ilicifolia Roivainen 1954

Nothofagus pumilio-Gaultheria mucronata Roivainen 1954

Nothofagus pumilio Seibert 1982

Nothofagus pumilio Collantes, Ontivero, Bianciotto 1990

\section{b.- arbustivas diversas}

Acaena splendens-Festuca pallescens Seibert 1979

Acaena splendens-Rumex acetosella Seibert 1979

Adesmia pinifolia-Mulinum spinosum Méndez 1971

Anarthrophyllum rigidum-Stipa speciosa Roig et Martínez Carretero 1992

Baccharido-Gaultherietum poeppigii Villagrán 1980

Berberis ilicifolia-Adenocaulon chilense Roivainen 1954

Bolax gummifera-Alopecurus antarcticus Roivainen 1954

Bolax gummifera-Empetrum rubrum Roig 2000

Chiliotrichum diffusum-Acaena magellanica

Roivainen 1954

Chiliotrichum diffusum-Alopecurus antarcticus Roivainan 1954

Chiliotrichum diffusum-Empetrum rubrum Roivainen 1954

Chiliotrichum diffusum-Festuca gracillima

Anchorena 1985

Chiliotrichum diffusum-Marsippospermum grandiflorum Roivainen 1954

Colletia spinosissima-Acaena splendens Seibert 1979

Colliguaja integerrima-Berberis grevilleana De Marco et al. 1993

Empetrum rubrum Dudley et Crow 1983 


\section{E. Martínez Carretero et al. - Prodromus Sinsistemático y Estudios Fitosociológicos}

Empetrum rubrum-Festuca gracillima Anchorena 1985

Empetrum rubrum-Viola columnaris Cabrera 1977

Ephedra ochreata-Aristida mendocina De Marco et al. 1993

Ephedra ochreata-Stipa speciosa De Marco et al. 1993

Fabiana patagonica-Mulinum spinosum De Marco et al. 1993

Fabiana peckii-Stipa chrysophylla De Marco et al. 1993

Gaultheria mucronata-Marsippospermum grandiflorum Dollenz 1985, 1986

Gaultheria mucronata-Viola magellanica Dollennz 1985-86

Gutierrezia spathulata-Bouteloua curtipendula De Marco et al. 1993

Junellia tridens-Stipa chrysophylla Anchorena 1985

Lomatia hirsuta-Acaena ovalifolia Seibert 1979

Mulinum spinosum-Acaena splendes Seibert 1979

Nassauvia glomerulosa-Stipa ameghinoi Anchorena 1985

Nassauvia glomerulosa-Poa dusenii Anchorena 1985

Nassauvia ulicina-Stipa dusenii Faggi 1983

Neosparton aphyllum-Acantholippia seriphioides Méndez 1971

Neosparton aphyllum-Fabiana patagonica Martínez Carretero et Roig 1992

Rosa eglanteria-Acaena splendens Seibert 1979

Schinus johnstonii-Lycium gilliesianum Gandullo et al. 1998

Senecio multicaulis-Grindelia chiloensis Méndez 1971

Senecio parodii Villagrán 1980

Senecio triodon-Perezia pedicularidifolia-Valeriana fonckii Villagrán 1980

Tetraglochin alatum-Gutierrezia spathulata De Marco et al. 1993

\section{c.- de pajonales, pastizales, praderas, etc.}

Acaena magellanica-Poa chrysantha Roivainen 1954 Acaena magellanica-Poa pratensis Roivainen 1954

Agrostis capillaris-Leptinella scariosa Dollenz 1985, 1986

Agrostis kuntzei-Polypogon monspeliensis Conticello, Bustamante et Cerazo 2008

Agrostio-Senecietum chilensis Villagrán 1980

Alopecurus antarcticus-Acaena magellanica

Roivainen 1954

Alopecurus antarcticus-Phleum alpinum Roivainen 1954
Amaranthus quitensis-Chenopodium hircinum Conticello, Bustamante et Cerazo 2008

Ambrosia tenuifolia Conticello, Bustamante et Cerazo 2008

Armeria maritima-Agrostis magellanica Roivainen 1954

Astelia pumila Dudley et Crow 1983

Blechnum penna-marina-Hierochlöe redolens Roivainen 1954

Blechnum penna-marina-Luzuriaga marginata Roivainen 1954

Blechnum penna-marina-Phleum alpinum Roivainen 1954

Bromus catharticus-Acaena poeppigiana Faggi 1983

Caltha appendiculata-Senecio trifurcatus-Aster vahlii Villagrán 1980

Caltha appendiculata Dudley et Crow 1983

Cardaria draba-Convolvulus arvensis Conticello, Bustamante et Cerazo 2008

Conium maculatum Conticello, Bustamante et Cerazo 2008

Deschampsia kingii-Acaena magellanica Roivainen 1954

Deyeuxia poaeoides-Acaena magellanica Roivainen 1954

Diplotaxis tenuifolia-Eruca sativa Conticello, Bustamante et Cerazo 2008

Empetrum rubrum-Pseudocyphellaria freycinetii Roivainen 1954

Empetrum rubrum-Trisetum phleoides Roivainen 1954

Festuca gracillima-Carex andina var. subabscondita Anchorena 1985

Festuca gracillima-Anarthrophyllum desideratum Faggi 1983

Festuca gracillima-Carex macloviana Anchorena 1985

Festuca gracillima-Nassauvia axillaris Faggi 1983

Festuca gracillima-Poa dusenii Faggi 1983

Festuca gracillima-Aster vahlii Anchorena 1985

Festuca gracillima-Rytidosperma virescens Anchorena 1985

Festuca monticola-Empetrum rubrum Villagrán 1980

Festuca pallescens-Acaena poeppigiana Anchorena 1985

Festuca pallescens-Hordeum comosum Montaldo 1976b

Festuca pallescens-Pernettya pumila var. crassifolia Cabrera 1977

Festuca pallescens-Poa dusenii Faggi 1983 
Festuca pallescens-Rytidosperma picta León et Aguiar 1985

Gaultheria myrtilloides Villagrán 1980

Hordeum murinum-Bromus catharticus Conticello, Bustamante et Cerazo 2008

Poa durifolia-Stipa speciosa Méndez 1971

Poa dusenii-Carex andina var. subabscondita Anchorena 1985

Poa flabellata Dudley et Crow 1983

Poa fuegiana-Senecio philippi Villagrán 1980

Poa robusta-Hierochlöe redolenz Dollenz 1985-86

Primula magellanica Villagrán 1980

Racomitrium lanuginosum Villagrán 1980

Ranunculus uniflorus-Perezia lactucoides Roivainen 1954

Ranunculus uniflorus-Stellaria debilis Roivainen 1954

Ranunculus peduncularis-Acaena magellanica Roivainen 1954

Ranunculus peduncularis-Phleum alpinum Roivainen 1954

Senecio parodii Villagrán 1980

Senecio smithii-Alopecurus antarcticus Roivainen 1954

Senecio smithii-Ranunculus uniflorus Roivainen 1954

Scirpus californicus Conticello, Bustamente et Cerazo 2008

Stipa chrysophylla-Carex patagonica Faggi 1983

Stipa speciosa var. manqueclensis-Panicum urvilleanum De Marco et al. 1993

Stipa speciosa-Rhodophiala mendocina Martínez Carretero et Roig 1992

Trifolium repens-Taraxacum officinale Conticello, Bustamante et Cerazo 2008

Trisetum phleoides-Gentianella magellanica Roivainen 1954

Viola tridentata-Phleum alpinum Roivainen 1954

\section{d.- halófilas}

Baccharis spartioides-Distichlis scoparia Therburg 1977

Chenopodium macrospermum-Ranunculus cymbalaria Martínez Carretero et Roig 1992

Distichlis scoparia-Prosopis strombulifera Gandullo et al. 1998

Distichlis spicata-Limonium brasiliense Therburg 1997

Frankenia juniperoides-Distichlis spicata Therburg 1997
Frankenia juniperoides-Heterostachys ritteriana Therburg 1977

Juncus arcticus-Taraxacum officinale Faggi 1983

Lepidophyllum cupressiforme-Puccinellia mendozinae Anchorena 1985

Sarcocornia ambigua-Limonium brasiliense Martínez Carretero et Roig 1992

Sarcocornia ambigua-Chenopodium macrospermum Therburg 1997

Sarcocornia ambigua-Heterostachys ritteriana Therburg 1997

\section{e.- psamófilas}

Atriplex lampa-Neosparton aphyllum Therburg 1997 Hyalis argentea var. latisquama-Grindelia chiloensis Martínez Carretero et Roig 1992

Neosparton aphyllum-Grindelia chiloensis De Marco et al. 1993

Panicum urvilleanum-Calycera spinulosa Martínez Carretero et Roig 1992

Sporobolus rigens-Poa lanuginosa Gandullo et al. 1998

Sporobolus rigens-Stipa chrysophylla Méndez 1971

Sporobolus rigens-Poa lanuginosa Martínez Carretero et Roig 1992

f.- palustres, lacustres, de vegas, turberas, etc. Acaena magellanica-Caltha sagittata Roivainen 1954

Acaena magellanica-Carex atropicta Roivainen 1954

Acaena magellanica-Drapetes muscosus Roivainen 1954

Acaena magellanica-Mnium rugicum Roivainen 1954

Alopecurus antarcticus-Brachythecium turgens Schwaar 1981

Alopecurus antarcticus-Calliergon sarmentosum Roivainen 1954

Alopecurus antarcticus-Deyeuxia poaeoides Roivainen 1954

Alopecurus antarcticus-Dicranoloma harioti Roivainen 1954

Alopecurus antarcticus-Perezia lactucoides Roivainen 1954

Alopecurus antarcticus-Tortula robusta Roivainen 1954

Astelia pumila-Donatia fascicularis Dollenz 19851986

Astelia pumila-Myrteola nummularia Roivainen 1954 


\section{E. Martínez Carretero et al. - Prodromus Sinsistemático y Estudios Fitosociológicos}

Astelia pumila-Gunnera lobata Roivainen 1954

Astelia pumila-Gunnera magellanica Roivainen 1954

Caltha appendiculata-Oreobolus obtusangulus

Roivainen 1954

Caltha sagittata-Alopecurus magellanicus

Roivainen 1954

Caltha sagittata-Cardamine glacialis Roivainen 1954

Caltha sagittata-Colobanthus subulatus Roivainen 1954

Caltha sagittata-Drapetes muscosus Roivainen 1954

Caltha sagittata-Epilobium australe Roivainen 1954

Carex atropicta-Brachythecium turgens Roivainen 1954

Carex atropicta-Acaena magellanica Roivainen 1954

Carex atropicta-Drepanochladus subjulaceaeus Roivainen 1954

Carex atropicta-Marsippospermum grandiflorum Roivainen 1954

Carex atropicta-Primula magellanica Roivainen 1954

Carex curta-Acaena magellanica Roivainen 1954

Carex curta-Alopecurus magellanicus Roivainen 1954

Carex curta-Bryum pseudotrichetrum Schwaar 1981

Carex curta-Caltha sagittata Schwaar 1981

Carex curta-Carex maclowiana Schwaar 1981

Carex curta-Deyeuxia poaeoides Roivainen 1954

Carex curta-Drepanochladus fluitans Roivainen 1954

Carex curta-Drepanochladus revolvens Schwaar 1981

Carex curta-Plantago barbata Roivainen 1954

Carex curta-Poa pratensis Roig 2000

Carex curta-Sphagnum fimbriatum Roivainen 1954

Cladonia aueri-Rostkovia magellanica Roivainen 1954

Carex darwinii-Agrostis magellanica Roivainen 1954

Carex decidua-Acaena magellanica Roivainen 1954

Carex decidua-Dicranoloma harioti Roivainen 1954

Carex decidua-Empetrum rubrum Roivainen 1954

Carex gayana-Colobanthus crassifolius Roivainen 1954

Carex gayana-Colobanthus quitensis Roivainen 1954

Carex gayana-Juncus scheuchzerioides Roivainen 1954

Carex gayana-Drepanochladus revolvens Roivainen 1954
Carex gayana-Mnium rugicum Roivainen 1954

Carex gayana-Drepanochladus subjulaceus Roivainen 1954

Carex gayana-Cinclidium stygium Roivainen 1954

Carex gayana var. densa-Colobanthus quitensis Anchorena 1985

Carex magellanica-Dicranoloma harioti Roivainen 1954

Carex magellanica-Dicranoloma harioti Schwaar 1981

Carex magellanica-Drepanochladus revolvens Schwaar 1981

Carex microglochin-Bryum ventricosus Roivainen 1954

Cornicularia aculeata-Chorizodontium sphagneticolum Roivainen 1954

Deyeuxia poaeoides-Anisothecium hookeri Roivainen 1954

Deyeuxia poaeoides-Carex gayana Roivainen 1954

Deyeuxia poaeoides-Drepanochladus subjulaceus Roivainen 1954

Deyeuxia poaeoides-Tortula robusta Roivainen 1954 Drapetes muscosus-Polytrichum alpestre Roivainen 1954.

Drepanochladus subjulaeus-Tortula robusta Roivainen 1954

Donatia fascicularis-Gunnera lobata Roivainen 1954

Donatia fascicularis-Empetrum rubrum Roivanien 1954

Donatia fascicularis-Gaultheria antarctica Roivainen 1954

Donatia fascicularis-Tetroncium magellanicum Roivainen 1954

Eriachaenium magellanicum-Carex subantarctica Roig et al. 1985a

Eleocharis pachicarpa-Plagiobothrys calandrinoides Roig et al. 1985a

Eleocharis tucumanensis-Plantago tubulosa Seibert et Menhofer 1991, 1992

Empetrum rubrum-Cladonia vicaria Roivainen 1954

Empetrum rubrum-Juncus scheuchzerioides Roivainen 1954

Empetrum rubrum-Pilgerodendron uviferum Dollenz 1985-1986

Gentianella magellanica-Carex atropicta Roivainen 1954

Gentianella magellanica-Carex decidua Roivainen 1954

Gunnera magellanica-Alopecurus antarcticus Roivainen 1954 
Gunnera magellanica-Hierochlöe redolens Roivainen 1954

Gunnera magellanica-Marsippospermum grandiflorum Roivainen 1954

Gunnera magellanica-Galium antarcticum Roivainen 1954

Gunnera magellanica-Mnium rugicum Roivainen 1954

Gunnera magellanica-Schizeilema ranunculus Roivainen 1954

Gunnera magellanica-Trisetum phleoides Roivainen 1954

Juncus arcticus-Hordeum halophyllum Anchorena 1985

Juncus scheuchzerioides-Caltha sagittata Roivainen 1954

Juncus arcticus-Deschampsia flexuosa Montaldo 1976a

Juncus arcticus-Juncus scheuchzerioides Anchorena 1985

Hordeum lechleri-Artemisia magellanica Anchorena 1985

Marsippospermum grandiflorum-Adenocaulon chilense Roivainen 1954

Marsippospermum grandiflorum-Alopecurus antarcticus Roivainen 1954

Marsippospermum grandiflorum-Blechnum penamarina Roivainen 1954

Marsippospermum grandiflorum-Empetrum rubrum Roivainen 1954

Marsippospermum grandiflorum-Gaultheria pumila Roivainen 1954

Marsippospermum grandiflorum-Hierochlöe redolenz Dollenz 1985-1986

Marsippospermum grandiflorum-Holcus lanatus Dollenz 1985-1986

Marsippospermum grandiflorum-Sphagnum magellanicum Roivainen 1954

Meesea trichodes-Drepanochladus subjulaceus Roivainen 1954

Plantago barbata-Nanodea muscosa Roivainen 1954

Polytrichum alpestre-Cladonia cornuta Roivainen 1954

Polytrichum alpestre-Empetrum rubrum Schwaar 1981

Polytrichum alpestre-Schizeilema ranunculus Roivainen 1954

Ranunculus uniflorus-Drepanochladus revolvens Schwaar 1981
Rostkovia magellanica-Plantago barbata Roivainen 1954

Rostkovia magellanica-Polytrichum alpestre Roivainen 1954

Rostkovia magellanica-Sphagnum fimbriatum Roivainen 1954

Sphagnum fimbriatum-Carex curta Roivainen 1954

Sphagnum fimbriatum-Carex magellanica Schwaar 1981

Sphagnum fimbriatum-Gunnera magellanica Roivainen 1954

Sphagnum fimbriatum-Rostkovia magellanica Roivainen 1954

Sphagnum fimbriatum-Carex curta Schwaar 1981

Sphagnum fimbriatum-Carex magellanica Roivainen 1954

Sphagnum fimbriatum-Tetroncium magellanicum Roivainen 1954

Sphagnum magellanicum-Carex magellanica Roivainen 1954

Sphagnum magellanicum-Colobanthus subulatus Roivainen 1954

Sphagnum magellanicum-Empetrum rubrum Roig 2000

Sphagnum magellanicum-Empetrum rubrum Roivainen 1954

Sphagnum magellanicum-Empetrum rubrum Schwaar 1981

Sphagnum magellanicum-Marsippospermum grandiflorum Roig 2000

Sphagnum magellanicum-Marsippospermum grandiflorum Dollenz 1985-86

Sphagnum magellanicum-Marsippospermum grandiflorum Schwaar 1981

Sphagnum magellanicum-Rostkovia magellanica Roig 2000

Sphagnum magellanicum-Rostkovia magellanica Roivainen 1954

Sphagnum magellanicum-Polytrichum alpestre Roivainen 1954

Sphagnum subfalcatulum-Drepanochladus fluitans Roivainen 1954

Tetroncium magellanicum-Caltha appendiculata Roivainen 1954

Tetroncium magellanicum-Carpha schoenoides Roivainen 1954

Tetroncium magellanicum-Dicranoloma harioti Roivainen 1954

Tetroncium magellanicum-Gaultheria pumila Roivainen 1954 


\section{E. Martínez Carretero et al. - Prodromus Sinsistemático y Estudios Fitosociológicos}

Tetronciummagellanicum-Sphagnummagellanicum Roivainen 1954

Trisetum lechleri-Scutellaria nummulariaefolia Anchorena 1985

Tropaeolum patagonicum-Plagiobothrys calandrinioides Roig et al. 1985a

g.- adventicias, ruderales, arvenses, etc.

Cardaria draba-Convolvulus arvensis Conticello 1998

Trifolium repens-Holcus lanatus Anchorena 1985

\section{2.- PROVINCIA PATAGÓNICA}

a.- de bosques, rodales diversos, etc.

Discaria trinervis-Cortaderia rudiuscula De Marco et al. 1993

Luma apiculata Eskuche 1998

\section{b.- arbustivas diversas}

Atriplex lampa-Chuquiraga avellanedae Cingolani, Bran, López et Ayesa 2000

Chuquiraga avellanedae-Nassauvia glomerulosa Soriano 1950

Colliguaja integerrima Soriano 1950

Colliguaja integerrima-Berberis grevilleana De Marco et al.1993

Ephedra ochreata-Aristida mendocina De Marco et al. 1993

Ephedra ochreata-Stipa speciosa De Marco et al. 1993

Fabiana patagonica-Mulinum spinosum De Marco et al. 1993

Fabiana peckii-Stipa chrysophylla De Marco et al. 1993

Gutierrezia spathulata-Bouteloua curtipendula De Marco et al. 1993

Mulinum spinosum-Stipa speciosa Cingolani, Bran, López et Ayesa 2000

Nassauvia glomerulosa Soriano 1950

Nassauvia glomerulosa-Azorella caespitosa Bran et al. 1985

Nassauvia glomerulosa-Nassauvia darwinii Bran et al. 1985

Nassauvia glomerulosa-Poa dusenii Bertiller 1993

Nassauvia glomerulosa-Senecio filaginoides-Stipa humilis Cingolani, Bran, López et Ayesa 2000

Nassauvietea glomerulosae Martínez Carretero 2004, nom. inval.

Nassauvia ulicina-Stipa dusenii Faggi 1983
Neosparton aphyllum-Grindelia chiloensis De Marco et al. 1993

Poa ligularis-Junellia mulinoides Cingolani, Bran, López et Ayesa 2000

Prosopis denudans-Atriplex lampa-Stipa humilis Cingolani, Bran, López et Ayesa 2000

Schinus johnstonii-Lycium gilliesianum Gandullo et al. 1998

Stipa humilis-Nassauvia glomerulosa Cingolani, Bran, López et Ayesa 2000

Tetraglochin alatum-Gutierrezia spathulata De Marco et al. 1993

Verbena tridens Faggi 1985a

c.- de pajonales, pastizales, praderas, etc.

Agropyron elongatum Cerazo et Conticello 2007

Brometum hordacei plantaginosum Ringuelet 1938, nom. inval.

Bromus catharticus Faggi 1985a

Centaurea melitensis Cerazo et Conticello 2008

Fabiana denudata (glerófitos) Gandullo et Faggi 2006

Festuca argentina-Rytidosperma virescens Bran et al. 1985

Festuca argentina-Senecio filaginoides Bran et al. 1985

Festuca argentina-Stipa brevipes Bran et al. 1985

Festuca monticola Soriano 1950

Festuca pallescens-Carex patagonica Bran et al. 1985

Festuca pallescens-Poa dusenii Faggi 1983

Festuca pallescens-Rytidosperma picta León et Aguiar 1985

Festuca pallescens-Viola maculata subsp. microphyllos Bran et al. 1985

Festuca pallescens-Viola maculata Bran et al. 1985

Festuco-Pernettyetum Cabrera et al. 1977

Festuco-Pernettyetum y Empetretum Cabrera et al. 1977

Haplopappus prunelloides (casmófitos) Gandullo et Faggi 2006

Poa pratensis-Bromus fonckii Eskuche 1998

Rhodophiala andicola (cormófitos) Gandullo et Faggi 2006

Scirpetum ripari chenopodiosum Ringuelet 1938, nom. inval.

Scirpetum olneyi hordaceosum Ringuelet 1938, nom. inval.

Senecio poeppigii (casmófitos orófilos) Gandullo et Faggi 2006 
Sisymbrio-Rapistretum Conticello et al. 1998, nom. inval.

Spartinetum coarctatae Cabrera 1941

Spartinetum maritimae scirposum Ringuelet 1938, nom. inval.

Spartinetum montevidensis salicorniosum Ringuelet 1938, nom. inval.

Stellaria media Cerazo et Conticello 2008

Stipa chrysophylla-Carex patagonica Faggi 1983

Stipa patagonica-Stipa humilis Soriano 1950

Stipa speciosa-Senecio filaginoides Bran et al. 1985

Stipa speciosa var. manqueclensis-Panicum urvilleanum De Marco et al. 1993

Distichlis scoparia-Prosopis strombulifera Gandullo et al. 1998

Juncus arcticus-Taraxacum officinale Faggi 1983

Sporobolus rigens-Poa lanuginosa Gandullo et al. 1998

\section{3.- PROVINCIA DE LA PAYUNIA}

\section{a.- arbustivas diversas}

Adesmia pinifolia-Mulinum spinosum Méndez 1971

Anarthrophyllum rigidum-Stipa speciosa Martínez Carretero et Roig 1992

Atriplex lampa-Neosparton aphyllum Therburg 1997

Atriplex boecherii Méndez 2005

Atriplex lampa Méndez 2005

Baccharis spartioides-Phragmites australis Martínez Carretero et Roig 1992

Baccharis spartioides-Distichlis scoparia Therburg 1997

Baccharis spartioides Méndez 2005

Chuquiraga erinacea Méndez 2005

Chuquiraga hystrix-Panicum urvilleanum Martínez Carretero et Roig 1992

Fabiana peckii-Acantholippia seriphioides Böcher et al. 1972

Frankenia juniperoides-Distichlis spicata Therburg 1997

Frankenia juniperoides-Heterostachys ritteriana Therburg 1997

Frankenia juniperoides Méndez 2005

Heterostachys ritteriana Méndez 2005

Hyalis argentea var. latisquama-Grindelia chiloensis Martínez Carretero et Roig 1992

Hyalis argentea-Stipa seciosa Martínez Carretero et Roig 1992.

Larrea divaricata-Acantholippia seriphioides Martínez Carretero et Roig 1992
Larrea divaricata Méndez 2005

Larrea nitida Méndez 2005

Mulinum spinosum-Adesmia pinifolia Méndez 1971

Neosparton aphyllum-Acantholippia seriphioides Méndez 1971

Neosparton aphyllum-Fabiana patagonica Martínez Carretero et Roig 1992

Neosparton aphyllum-Grindelia chiloensis Böcher et al. 1972.

Neosparton aphyllum-Panicum urvilleanum

Martínez Carretero et Roig 1992

Neosparton aphyllum Méndez 2005

Sarcocornia ambigua-Limonium brasiliense Martínez Carretero et Roig 1992

Sarcocornia ambigua-Chenopodium macrospermum Therburg 1997

Sarcocornis ambigua-Heterostachys ritteriana Therburg 1997

Sarcocornia perennis Méndez 2005

Suaeda divaricata Méndez 2005

Senecio multicaulis-Grindelia chiloensis Méndez 1971

Senecio subulatus Méndez 2005

\section{b.- pastizales, praderas herbáceas, etc.}

Cressa truxillensis Méndez 2005

Distichlis spicata-Limonium brasiliense Therburg 1997

Distichlis spicata-Sarcocornia perennis Martínez Carretero et Roig 1992

Distichlis spp. Méndez 2005

Limonium brasiliense Méndez 2005

Panicum urvilleanum-Calycera spinulosa Martínez Carretero et Roig 1992

Panicum urvilleanum-Poa lanuginosa Martínez Carretero et Roig 1992

Panicum urvilleanum Méndez 2005

Poa durifolia-Stipa speciosa Méndez 1971

Poa durifolia-Stipa chrysophylla Méndez 1971

Stipa speciosa-Rhodophiala mendocina Martínez Carretero et Roig 1992

Sporobolus rigens-Stipa chrysophylla Méndez 1971

Sporobolus rigens-Poa lanuginosa Martínez Carretero et Roig 1992

Sporobolus rigens-Panicum urvilleanum Martínez Carretero et Roig 1992

Sporobolus rigens Méndez 2005

Stipa speciosa-Senna arnottiana Martínez Carretero et Roig 1992 


\section{E. Martínez Carretero et al. - Prodromus Sinsistemático y Estudios Fitosociológicos}

c.- lacustres, palustres, de vegas, etc.

Azolla filiculioides Méndez 2005

Chara australis Méndez 2005

Chenopodium macrospermum-Ranunculus

cymbalaria Martínez Carretero et Roig 1992

Cortaderia rudiuscula Méndez 2005

Eleocharis albibracteata Méndez 2005

Juncus articus Méndez 2005

Phragmites australis-Muhlenbergia asperifolia

Martínez Carretero et Roig 1992

Phragmites australis Méndez 2005

Rorippa nasturtium-aquaticum Méndez 2005

Schoenoplectus californicus Méndez 2005

Schoenoplectus pungens Méndez 2005

Senecio bonariensis Méndez 2005

Typha dominguensis Méndez 2005

Zannichellia palustris Méndez 2005

\section{II.-Territorio Andino}

(Provincias Andina, de la Puna y del Cardonal)

\section{4.-PROVINCIAANDINA}

a.- de bosques, rodales diversos, etc.

Adesmia schneideri-Schinus roigii Méndez 1986

Nassauvia revoluta Méndez 2014

\section{b.- arbustivas diversas}

Adesmia pinifolia-Adesmia aegiceras Ambrosetti et al. 1986; Martínez Carretero et Méndez 1992

Adesmia digitata-Poa holciformis Ambrosetti et al. 1986

Adesmia aegiceras-Stipa chrysophylla Martínez Carretero et Méndez 1992

Adesmia aegiceras-Gayophyton micranthum Méndez 1994

Adesmia aegiceras-Poa holciformis Martínez Carretero et Méndez 1992

Adesmia aegiceras-Perezia carthamoides Méndez 1994

Adesmia aegiceras-Tropaeolum polyphyllum Ambrosetti et al. 1986

Adesmia aegiceras-Stipa chrysophylla Méndez 1994

Adesmia obovata Méndez 2014

Adesmia pinifolia-Poa holciformis Méndez 1994

Adesmia pinifolia-Haplopappus scrobiculatus

Méndez 1994

Adesmia pinifolia Méndez 2014

Adesmia volkmannii-Schinus odonelli Méndez 2014

Azorella monantha Méndez 2014
Baccharis polifolia-Junellia spathulata Méndez 1986

Berberis empetrifolia-Stipa chrysophylla Méndez 1994

Chuquiraga oppositifolia Méndez 2014

Colliguaha integérrima-Schinus polygamus Méndez 2014

Empetrum rubrum Méndez 2014

Ephedra andina-Acaena magellanica Méndez 1994

Fabiana imbricata Méndez 2014

Malesherbia lirana var. subglabrifolia-Adesmia aegiceras Dalmasso 1997

Nassauvia lagascae-Menonvillea cuneata Martínez Carretero et Méndez 1992

Neosparton ephedroides Méndez 2014

Phacelia secunda-Adesmia subterranea Méndez 1994 Saxifraga magellanica-Notholaena nivea Gutte 1986 Senecio filaginoides-Cassia arnottiana Méndez 1986 Senecio gilliesii-Arenaria serpens Méndez 1986

Senecio subulatus-Lathyrus macropus Méndez 1986

Senecio subumbellatus-Senecio filaginoides Méndez 1986

Tropaeolum polyphyllum-Bromus setifolius Méndez 1994

Tropaeolum polyphyllum-Convolvulus arvensis Méndez 1994

Viguiera tucumanensis-Adesmia cytisoides Mendiola 2004

c.- de pajonales, pastizales, praderas, etc.

Aristida spp.-Eragrostis nigricans Mendiola 2004

Bouteloua simplex-Lycurus phleoides Gutte 1986

Festuca kuntziana-Taraxacum gilliesii Méndez 1986

Festuca kurtziana Méndez 2014

Pappostipa speciosa Méndez 2014

Penisetum chilense Mendiola 2004

Poa holciformis Méndez 2014

Stipa speciosa var. media-Boopis gracilis Méndez 1986

\section{d.-lacustres, palustres, de vegas etc.}

Acaena magellanica-Plantago barbata Méndez 1986 Acaena magellanica-Tropaeolum polyphyllum Méndez 1994

Carex gayana-Eleocharis albibracteata Méndez 1994

Carex Ontivero et Martínez Carretero 2013

Epilobium nivale-Veronica anagallis-aquatica Méndez 1994

Juncus arcticus-Eleocharis albibracteata Méndez 1986

Juncus arcticus Ontivero et Martínez Carretero 2013

Juncus balticus Ontivero et Martínez Carretero 2013 
Juncus balticus Méndez 2014

Mimulus luteus-Veronica anagallis-aquatica Méndez 1986

Myriophyllum elatinoides Méndez 1986

Ochetophila nana Méndez 2014

Oxychloe bisexualis-Vicia patagonica Méndez 1986

Patosia clandestina Ontivero et Martínez Carretero 2013

Patosia clandestina Méndez 2014

Potamogeton pectinatus Ontivero et Martínez Carretero 2013

Ranunculus cymbalaria-Carex gayana Méndez 1986

Ranunculus peduncularis Méndez 2014

Ranunculus sp.-Trifolium repens Méndez 1994

Schoenoplectus californicus Méndez 1986

Veronica anagallis-aquatica-Nasturtium officinale

Gutte 1986

\section{e.- riparias}

Cortaderia rudiuscula-Azorella gilliesii Méndez 1986

Cortaderia rudiuscula Méndez 2014

\section{5.-PROVINCIA DE LA PUNA}

a.- de bosques, rodales diversos, etc.

Baccharis salicifolia-Schinus areira Ruthsatz 1977

Polylepis tomentella-Adesmia spinosissima Ruthsatz 1977

\section{b.- arbustivas diversas}

Adesmia horrida-Senecio uspallatensis Ambrosetti et al. 1986

Adesmia pinifolia Martínez Carretero, Dalmasso, Márquez et Martinelli 2009

Adesmia subterranea-Stipa frigida Martínez Carretero 1997

Adesmia subterranea Martínez Carretero, Dalmasso, Márquez et Martinelli 2009

Acantholippia salsoloides Borgnia et al. 2006

Acantholippia punensis-Lycium chanar Martínez Carretero 1997

Artemisia echegarayi-Senecio uspallatensis Martínez Carretero 1994

Artemisia copa-Adesmia horrida Tálamo et al. 2010

Baccharis grisebachii-Baccharis boliviensis Ruthsatz 1977

Baccharis incarum-Artemisia mendozana var. paramilloensis Dalmasso et al. 1999

Baccharis incarum-Larrea nitida Martínez Carretero 1994
Baccharis incarum-Tephrocactus melanacanthus Ruthsatz 1977

Baccharis incarum var. lejia-Junellia seriphioides Ruthsatz 1977

Bulnesia schickendantzii-Parodia setigera Ruthsatz 1977

Calycera pulvinata $f$. crenata-Descurainia sp. Ruthsatz 1977

Cortaderia rudiuscula-Juncus arcticus var. mexicanus Roig et Martínez Carretero 1998

Cristaria andicola-Adesmia subterranea Martínez Carretero 1997

Ephedra breana-Junellia erinacea Ambrosetti et al. 1986

Fabiana densa-Adesmia horrida-Tetraglochin cristatum-Pseudobaccharis boliviensis Cabrera 1948b Fabiana densa-Baccharis boliviensis Ruthsatz 1977

Fabiana densa-Aristida humilis Ruthsatz 1977

Fabiana densa-Baccharis boliviensis Werner 1978

Fabiana densa Borgnia et al. 2006

Fabiana densa-Adesmia horrida Tálamo et al. 2010

Fabiana denudata-Baccharis boliviensis var. latifolia Ruthsatz 1977

Junellia asparagoides-Proustia cuneifolia Ruthsatz 1977

Lampaya hieronymi-Adesmia horrida Martínez Carretero 1997

Larrea divaricata-Lycium vergarae Dalmasso et al. 1999

Larrea divaricata Martínez Carretero, Dalmasso, Márquez et Martinelli 2009

Lepidophyllum tola-Lepidophyllum phyliciforme Cabrera 1948b

Lycium fuscum-Chuquiraga erinacea Dalmasso et al. 1999

Lycium fuscum-Fabiana patagonica Ambrosetti et al. 1986

Lycium fuscum-Junellia asparagoides Martínez Carretero 1994

Lycium vergarae-Larrea divaricata Martínez Carretero 1994

Nassauvia axillaris-Mulinum spinosum Ambrosetti et al. 1986.

Nicotiana corymbosa-Chenopodium ruiz-lealii Roig et Martínez Carretero 1992

Nototriche friesii-Senecio aquilaris Ruthsatz 1977

Parastrephia lepidophylla-Tarasa tarapacana Ruthsatz 1977

Parastrephia quadrangularis Tálamo et al. 2010

Plazia daphnoides-Menodora decemfida Roig et Martínez Carretero 1998 


\section{E. Martínez Carretero et al. - Prodromus Sinsistemático y Estudios Fitosociológicos}

Plazia daphnoides-Salvia gilliesii Martínez Carretero 1994

Plazia daphnoides-Stipa neaei Dalmasso et al. 1999

Poa gymnantha-Senecio adenophyllus Ruthsatz 1977

Sarcocornia pulvinata-Distichlis humilis Ruthsatz 1977, em.

Trycicla spinosa Martínez Carretero, Dalmasso, Márquez et Martinelli 2009

Werneria pseudodigitata-Alchemilla sp. Ruthsatz 1977

c.- de pajonales, pastizales, praderas herbáceas, etc.

Aristida humilis-Astragalus micranthellus Ruthsatz 1977

Chiliotrichiopsis keideli-Mutisia ledifoliaSatureja parvifolia Cabrera 1948b

Distichlis scoparia-Triglochin concinna Martínez Carretero 1997

Distichia muscoides Seibert 1993

Calamagrostis cabrerae Tálamo et al. 2010

Festuca scirpifolia Cabrera 1948b

Festuca orthophylla-Calamagrostis cabrerae-

Stipa hieronymusi Cabrera 1948b

Festuca orthophylla-Deyeuxia eminens var. fulva

Martínez Carretero 1997

Festuca sp.-Lycium chanar-Junellia seriphioides

Borgnia et al. 2006

Muhlenbergia fastigiata-Bouteloua simplex

Cabrera 1948b

Lampaya castellani Cabrera 1948b

Pseudobaccharis acaulis-Salicornia pulvinata-

Distichlis humilis Cabrera 1948b

Panicum chloroleucum Cabrera 1948b

Panicum chloroleucum Borgnia et al. 2006

Panicum chloroleucum-Acantholippia salsoloides Borgnia et al. 2006

Pappostipa hieronymusii Tálamo et al. 2010

Pennisetum chilense Cabrera 1948a

Sporobolus rigens-Fabiana atacamensis Cabrera 1948b

Sporobolus rigens f. atacamensis Ruthsatz 1977

Sporobolus rigens Borgnia et al. 2006

Stipa scirpea-Bromus brevis Dalmasso et al. 1999

Stipa chrysophylla-Adesmia subterranea Martínez Carretero 1997

Stipa frigida-Fabiana bryoides Martínez Carretero 1997

Stipa frigida Martínez Carretero Dalmasso Márquez et Martinelli 2009

Stipa vaginata f. rigida-Junellia uniflora Martínez Carretero 1994
Stipa scirpea-Adesmia horrida Martínez Carretero 1994

d.- palustres, lacustres, de vegas, etc.

Alchemilla pinnata-Eleocharis albibracteata Ruthsatz 1977

Carex gayana-Deyeuxia velutina Martínez Carretero 1997

Carex incurva Martínez Carretero, Dalmasso, Márquez et Martinelli 2009

Distichlis humilis-Amphiscirpus nevadensis Borgnia et al. 2006

Festuca argentinensis Borgnia et al. 2006

Frankenia triandra-Sarcocornia pulvinata Borgnia et al. 2006

Hypsela oligophylla Ruthsatz 1977

Juncus arcticus Martínez Carretero, Dalmasso, Márquez et Martinelli 2009

Juncus balticus Martínez Carretero, Dalmasso, Márquez et Martinelli 2009

Oxychloe andina-Carex incurva-Scirpus atacamensis Cabrera 1948b

Oxychloe andina-Scirpus atacamensis Martínez Carretero 1997

Patosia clandestina Martínez Carretero, Dalmasso, Márquez et Martinelli 2009

Potamogeton strictus-Myriophyllum elatinoides Cabrera $1948 \mathrm{~b}$

Potamogeton pectinatus Martínez Carretero, Dalmasso, Márquez et Martinelli 2009

Ranunculus mandonianus-Deyeuxia eminens Martínez Carretero 1997

Scirpus atacamensis-Juncus depauperatus-Plantago tubulosa Cabrera 1948b

Triglochin cfr. palustris-Puccinellia cfr. frigidaMuhlenbergia peruviana Borgnia et al. 2006

Werneria poposa Cabrera 1948b

\section{6.- PROVINCIA DEL CARDONAL}

a.- de bosques, rodales diversos, etc.

Prosopis alba-Trichocereus pasacana Ruthsatz 1977 Prosopisetum albae Ruthsatz 1977, prov.

Schinus areira-Lycium tenuispinosum Ruthsatz 1977. Schinus fasciculata-Colliguaja integerrima Roig et Ambrosetti 1971

\section{b.- arbustivas diversas}

Abromeitiella brevifolia-Parodia setifera Ruthsatz 1977

Abromeitiella lorentziana-Nothoscordum sp. Ruthsatz 1977 
Adesmia trijuga-Eupatorium buniifolium Roig et Martínez Carretero 1992

Adesmia trijuga-Hyalis argentea var. argentea Martínez Carretero 1993

Adesmia uspallatensis-Junellia asparagoides Ambrosetti et al. 1986

Adesmia uspallatensis-Junellia asparagoides Roig 1989

Artemisia echegarayi-Sisymbrium arnottianum Dalmasso et al. 1999

Bulnesietum schickendantzii Ruthsatz 1977, prov.

Bulnesia schickendantzii-Parodia setifera Ruthsatz 1977

Colliguaja-Baccharis pulchella Roig et Ambrosetti 1971; Roig 1973b

Colliguaja-Junellia scoparia Roig 1989

Colliguaja integerrima-Lobivia formosa Roig 1976

Colletia spinosissima-Berberis grevilleana Martínez

Carretero 1993

Colletia sipinosissima-Eupatorium patens Roig 1989

Dolychlasium lagascae-Lobivia formosa Dalmasso et al. 1999

Eupatorium buniifolium-Bredemeyera colletioides Martínez Carretero 1993

Eupatorium buniifolium-Colliguaja integerrima Dalmasso et al. 1999

Gochnatia glutinosa-Caesalpinia pumila Ruthsatz 1977

Gochnato glutinosae-Caesalpinietum pumilae Ruthsatz 1977, prov.

Hyalis argentea-Eupatorium patens Roig 1989

Junellia asparagoides-Mulinum spinosum Roig 1989

Junellia scoparia-Artemisia mendozana Roig 1989 Junellia scoparia-Berberis grevilleana Roig 1989 Junellia scoparia-Schinus fasciculata Roig 1989

Lobivia formosa-Larrea nitida Dalmasso et al. 1999 Menodora decemfida-Artemisia mendozana Roig 1971, 1973b; Ambrosetti et al. 1986

Menodora decemfida-Dipyrena glaberrima Roig 1989

Mulinum spinosum-Poa resinulosa Roig 1989

Schinus fasciculata-Dipyrena glaberrima Ambrosetti et al. 1986

Suaeda divaricata-Lycium tenuispinosum Ruthsatz 1977

Tricycla spinosa-Schinus fasciculata Roig 1989 c.- de pajonales, pastizales, praderas, etc.

Stipa tenuissima-Adesmia schneideri González Loyarte 1989a

Stipa tenuissima-Junellia juniperina González Loyarte 1989a

Stipa tenuissima-Chenopodium ambrosioides González Loyarte 1989a

Stipa tenuissima-Nassauvia axillaris González Loyarte 1989a

\section{III.-Territorio CENTRAL}

(Provincias Chaqueña, Pampeana, del Espinal y del Monte)

\section{7.-PROVINCIA CHAQUEÑA}

a.- de bosques, rodales diversos, etc.

Acacia aroma-Prosopis flexuosa Carranza et al. 1993 Acacia caven-Aloysia gratissima Borisov et al. 1991 Acacia caven-Baccharis articulata Vischi et al.1999. Acacia caven-Eupatorium buniifolium Vischi et al. 1999

Acacia caven Carnevali 1994

Acacia caven Hilgert et al. 2003

Aspidosperma quebracho-blanco-Mimozyganthus carinatus Ragonese 1951, Martínez Crovetto et Piccinini 1977

Aspidosperma quebracho-blanco con Larrea cuneifolia Ragonese et Castiglioni 1970, Eskuche 1984 Aspidosperma quebracho-blanco-Bromelia serra Martínez Crovetto 1980b

Aspidosperma quebracho-blanco Vallejos 1991

Aspidosperma quebracho-blanco Carranza et al. 1993 Aspidosperma quebracho-blanco-Schinopsis haenkeana Cabido et al. 1994

Aspidosperma quebracho-blanco-Prosopis flexuosa Cabido et al. 1994

Aspidosperma quebracho-blanco-Stetsonia coryne Cabido et al. 1994

Astronium balansae Carnevali 1994

Astronium balansae-Diplokeleba floribunda Maturo et al. 2005

Butia paraguayensis-Butia yatay Carnevali 1994

Butia yatay-Acacia bonariensis Martínez Crovetto et Piccinini 1950

Butia yatay-Acanthospermum australe Martínez Crovetto et Piccinini 1950

Butia yatay-Axonopus compressus Martínez Crovetto et Piccinini 1950 


\section{E. Martínez Carretero et al. - Prodromus Sinsistemático y Estudios Fitosociológicos}

Butia yatay-Paspalum notatum Carnevali 1994

Butia yatay-Paspalum quadrifarium Martínez Crovetto et Piccinini 1950

Butia yatay-Phyla canescens Martínez Crovetto et Piccinini 1950

Butia yatay-Sapium haematospermum Martínez Crovetto et Piccinini 1950

Butia yatay-Butia paraguayensis Carnevali 1994

Butia yatay-Sapium haematospermum Batista et al. 2014

Butia yatay-Sapium haematospermum-Schinus longifolius Batista et al. 2014

Butia yatay-Sebastiana commersoniana Batista et al. 2014

Calycophyllum multiflorum Adámoli et al. 1972

Celtis spinosa-Cestrum parqui Borisov et al. 1991

Copernicia australis Carnevali 1994

Copernicia australis Maturo et al. 2005

Enterolobium contortisiliquum-Patagonula americana Maturo et al. 2005

Erythrina crista-galli Carnevali 1994

Fagara coco-Lepechinia floribunda Vischi et al. 1999

Geoffroea decorticans-Grabowskia duplicata Cantero et al. 1996

Geoffroea subtropicalis Martínez Carretero 2015

Holocalyx balansae-Ficus luschnathiana-Cordia americana Prado et al. 1989

Lithraea ternifolia-Acacia caven Vischi et al. 1999

Lithraea ternifolia-Fagara coco Cabido et al. 1991

Lithraea ternifolia-Lepechinia floribunda Borisov et al. 1991

Lithraea ternifolia Carranza et al. 1993

Phyllostylon rhamnoides-Cathormion polyanthum Adámoli et al. 1972

Polylepis australis Cabido et Acosta 1985

Prosopis affinis-Astronium balansae Maturo et al. 2005

Prosopis flexuosa-Aspidosperma quebracho blanco Borisov et al.1991

Prosopis flexuosa-Aspidosperma quebracho-blanco Carranza et al. 1993

Prosopis flexuosa-Opuntia sulphurea Carranza et al. 1993

Prosopis flexuosa-Aspidosperma quebracho-blanco Cabido et al. 1994

Prosopis flexuosa-Mimozyganthus carinatus Cabido et al. 1994

Prosopis hasslerii-Prosopis affinis Eskuche 1986

Prosopis nigra-Aspidosperma quebracho-blancoCopernicia australis Carnevali 1994
Prosopis nigra-Parkinsonia aculeata Martínez et Prado 2013

Prosopis ruscifolia Adámoli et al. 1972

Prosopis spp. Hilgert et al. 2003

Ruprechtia laxiflora-Eugenia uniflora Lewis et al. 1994

Salix humboldtiana Adámoli et al. 1972

Salix humboldtiana Carnevali 1994

Schinopsis balansae-Astronium balansae Eskuche 1984

Schinopsis balansae-Astronium balansaeDiplokeleba floribunda Eskuche 1984

Schinopsis balansae-lorentzii Eskuche 1984

Schinopsis balansae Carnevali 1994

Schinopsis balansae-Acacia praecox Lewis et al. 1994

Schinopsis balansae-Acacia praecox Lewis et al. 1997

Schinopsis balansae Hilgert et al. 2003

Schinopsis balansae Marino et Pensiero 2003

Schinopsis balansae-Prosopis spp.-Aspidosperma quebracho-blanco Maturo et al. 2005

Schinopsis haenkeana-Lithraea ternifolia Cabrera 1976; Luti et al. 1979; Eskuche 1984

Schinopsis haenkeana-Aspidosperma quebrachoblanco Cabido et al. 1991

Schinopsis haenkeana-Ruprechtia apetala Cabido et al. 1991

Schinopsis haenkeana Carranza et al. 1993

Schinopsis haenkeana-Aspidosperma quebrachoblanco Carranza et al. 1993

Schinopsis lorentzii-Aspidosperma quebrachoblanco Adámoli et al. 1972

Schinopsis lorentzii-Prosopis nigra-Anadenanthera macrocarpa Adámoli et al. 1972

Schinopsis lorentzii-Aspidosperma quebrachoblanco con Cereus Eskuche 1984

Schinopsis lorentzii-Aspidosperma quebrachoblanco con Bulnesia sarmientoi Morello et Saravia Toledo 1959, Ragonese et Castiglioni 1970; Eskuche 1984

Schinopsis marginata-Prosopis spp.-Celtis tala Martínez et Prado 2013

Terminalia australis Batista et al. 2014

Tessaria integrifolia Carnevali 1994

\section{b.- arbustivas diversas}

Acacia aroma Adámoli et al. 1972

Allenrolfea vaginata Pire et Lewis 1993-1994

Allenrolfea vaginata-Heterostachys ritteriana Cabido et al. 1994 
Apurimacia dolichocarpa Funes et Cabido 2008

Atriplex suberecta Iriart 2016

Atriplex undulata-Cyclolepis genistoides Cantero et al. 1996

Baccharis salicifolia Adámoli et al. 1972

Berberis hieronymi-Satureja odora Cabido et al. 1990

Bromelia serra-Aechmea distichantha Martínez Crovetto 1980b

Bulnesia sarmientoi Adámoli et al. 1972

Celtis pallida-Achatocarpus praecox Lewis et al. 1997

Cyclolepis genistoides-Lycium americanum Lewis et Pire 1996

Eupatorium ivifolium-Setaria rosengurtii Marino et Pensiero 2003

Flourensia oolepis-Dyckia floribunda Cabido et al. 1991

Flourensia oolepis Carranza et al. 1993

Heterothalamus alienus Galera 1980

Heterothalamus alienus-Croton argentinus Cabido et al. 1990

Heterostachys ritteriana Ragonese et Piccinini 1977

Imperata brasiliensis-Elionurus muticus Maturo et al. 2005

Larrea cuneifolia Ragonese et Piccinini 1977

Larrea divaricata Carranza et al. 1993

Larrea divaricata Cabido et al. 1994

Larrea divaricata-Mimozyganthus carinatus Cabido et al. 1994

Polylepis australis Cabido et Acosta 1985

Stetsonia coryne Cabido et al. 1994

Suaeda divaricata Adámoli et al. 1972

Tessaria dodoneifolia-Schinus fasciculata Maturo et al. 2005

Tessaria integrifolia Adámoli et al. 1972

c.- de pajonales, pastizales, praderas, etc.

Alchemilla pinnata-Hypoxis humilis Cabido et al. 1989

Andropogon lateralis Vallejos 1991

Andropogon lateralis Carnevali 1994

Andropogon lateralis-Paspalum notatum Carnevali 1994

Andropogon lateralis-Rhynchospora pringlei Carnevali 1994

Andropogon lateralis-Sorghastrum agrostoides Carnevali 1994

Andropogon lateralis-Schizachyrium spicatum Maturo et al. 2005
Andropogon lateralis-Panicum glabripes Batista et al. 2014

Aristida venustula Vallejos 1991

Aristida venustula Carnevali 1994

Aristida jubata-Axonopus compressus Carnevali 1994

Baccharis dracunculifolia Batista et al. 2014

Bothriochloa saccharoides Galera 1980

Bouteloua aristidoides-Gomphrena martiana Carranza et al. 1993

Bouteloua megapotamica-Gomphrena pulchella Cabido et al. 1989

Bromelia serra-Lantana sp. Marino et Pensiero 2003

Chloris canterai Cantero et al. 1996

Cortaderia selloana-Aster squamatus Lewis et al. 1990

Cynodon dactylon-Gerardia communis Lewis et al. 1987

Cynodon dactylon-Hordeum stenostaschys Cantero et al. 1996

Cynodon dactylon Hilgert et al. 2003

Deyeuxia hieronymi-Stipa amethystina Cabido et al. 1989

Distichletum Collantes et Lewis 1980

Distichlis scoparia Cantero et al. 1996

Distichlis spicata Cantero et al. 1996

Eleocharis viridans-Coleataenia prionitis Lewis et al. 1987

Elionorus muticus-Spartina argentinensis Eskuche 1984

Elionorus muticus Lewis et al. 1990

Elionorus muticus Vallejos 1991

Elionorus muticus Carnevali 1994

Elionurus muticus-Paspalum notatum Carnevali 1994

Eragrostis hypnoides-Paspalum almum Lewis et al. 1987

Eupatorium hecatanthum-Ambrosia elatior Lewis et al. 1987

Festuca hieronymi Galera 1980

Festuca lilloi-Deyeuxia hieronymi Cabido et al. 1989

Muhlenbergia peruviana-Hypoxis humilis Cabido et al. 1989

Panicum elephantipes Adámoli et al. 1972

Panicum prionitis Adámoli et al. 1972

Panicum prionitis Vallejos 1991

Panicum prionitis Carnevali 1994

Panicum grumosum-Panicum rivulare Carnevali 1994

Pappophorum caespitosum-Muhlenbergia asperifolia Cantero et al. 1996 


\section{E. Martínez Carretero et al. - Prodromus Sinsistemático y Estudios Fitosociológicos}

Pappophorum pappiferum-Eragrostis lugens Lewis et al. 1990

Paspalum vaginatum-Cyperus corymbosusPaspalum quadrifarium Cantero et al. 1996

Paspalum intermedium-Rhynchospora scutellata Maturo et al. 2005

Paspalum acuminatum Carnevali 1994

Paspalum durifolium-Andropogon lateralis Carnevali 1994

Paspalum durifolium-Panicum sabulorum Carnevali 1994

Paspalum notatum-Axonopus spp. Vallejos 1991

Paspalum notatum-Axonopus affinis Carnevali 1994

Paspalum notatum-Desmodium incanum-Vernonia chamaedrys Carnevali 1994

Paspalum notatum-Aristida jubata Martínez Crovetto 1980a

Paspalum notatum-Eupatorium subhastatum Martínez Crovetto 1980b

Paspalum notatum-Eupatorium subhastatum Martínez Crovetto 1978

Paspalum notatum-Vernonia chamaedrys Vallejos 1993

Paspalum quadrifarium-Eryngium agavifolium Cabido et al. 1989

Pennisetum frutescens Adámoli et al. 1972

Pennisetum frutescens-Setaria pampeana Lewis et al. 1990

Schizachyrium sp.-Eupatorium squarroso-ramosum Lewis et al. 1990

Scirpo-Paspaletum Collantes et Lewis 1980

Sebastiana commersoniana-Blepharocalyx salicifolius Batista et al. 2014

Sorghastrum agrostoides Carnevali 1994

Sorghastrum agrostoides-Rhynchopora tenuis Carnevali 1994

Sorghastrum agrostoides-Axonopus affinis Carnevali 1994

Sorghastrum agrostoides-Rhynchospora emaciata Carnevali 1994

Sorghastrum pellitum-Stipa filiculmis Cabido et al. 1989

Sorghastrum pellitum-Stipa flexibarbata Cabido et al. 1989

Sorghastrum pellitum-Stipa flexibarbata Cabido et al. 1990

Sorghastrum setosum-Leersia hexandra Maturo et al. 2005

Spartina argentinensis Lewis et al. 1990

Spartina argentinensis Carnevali 1994
Spartina argentinensis-Panicum prionitis Hilgert et al. 2003

Spartina densiflora Cantero et al. 1996

Spartinetum Collantes et Lewis 1980

Sporobolo-Chloretum Collantes et Lewis 1980

Sporobolus pyramidatus-Sesuvium portulacastrum

Lewis et al. 1987

Stipa tenuissima Galera 1980

Stipa filiculmis-Stipa tenuissima Cabido et al. 1989

Stipa neesiana var. sublaevis-Agrostis montevidensis Cabido et al. 1989

Stipa tenuissima-Cestrum parqui Borisov et al. 1991

Stipa trichotoma-Stipa tenuissima-Stipa papposa Cantero et al. 1996

Stipo-Bothriochletum Collantes et Lewis 1980

Stipo-Sporoboletum Collantes et Lewis 1980

Stipo-Caricetum Collantes et Lewis 1980

Verbesina encelioides-Parthenium hysterophorus Carranza et al. 1993

Verbesina encelioides Iriart 2016

d.- lacustres, palustres, de vegas, etc.

Cyperus giganteus Carnevali 1994

Cyperus giganteus (poblaciones puras) Maturo et al. 2005

Cyperus prolixus-Cyperus virens Marino et Pensiero 2003

Echinochloa crusgalli-Solanum glaucophyllum Lewis et al. 1990

Echinochloa helodes Cantero et al. 1996

Eleocharis dombeyana-Hordeum stenostachys Cabido et al. 1989

Eleocharis macrostachya-Paspalidium paludivagum Lewis et al. 1990

Eleocharis nodulosa-Eleocharis fistulosa Carnevali 1994

Luziola peruviana-Jaborosa integrifolia Lewis et al. 1990

Luziola peruviana-Eleocharis nodulosa Carnevali 1994

Paspalum dilatatum-Eleocharis dombeyana Cabido et al. 1989

Paspalum intermedium-Aeschynomene rudis Lewis et al. 1990

Schoenoplectus californicus Hilgert et al. 2003

Schoenoplectus californicus (poblaciones puras)

Maturo et al. 2005

Scirpus californicus Lewis et al. 1990

Scirpus californicus Carnevali 1994 
Scirpus californicus-Baccharis juncea-Typha latifolia Cantero et al. 1996

Thalia geniculata (poblaciones puras) Maturo et al. 2005

Typha domingensis-Ammania friesii Lewis et al. 1990

Typha dominguensis Hilgert et al. 2003

Zizaniopsis bonariensis Carnevali 1994

\section{e.- halófilas}

Allenrolfea patagonica-Heterostachys ritteriana

Ragonese 1951

Cereus coryne-Maytenus vitis-idaea Ragonese 1951

Chenopodium ambrosioides-Chenopodium album Lewis et al. 1990

Festuca hieronymi Luti et al. 1979

Heterostachys ritteriana-Cressa truxillensis Lewis et al. 1990

Heterostachys ritteriana Cantero et al. 1996

Pappophorum mucronulatum-Baccharis notosergila Lewis et al. 1990

Polygonum aviculare-Diplachne uninervia Lewis et al. 1990

Samolus valerandi-Abobra tenuifolia Lewis et al. 1990

Spartina argentinensis-Aster squamatus Lewis et al. 1990

Spartina densiflora-Chenopodium macrospermum Lewis et al. 1990

Sarcocornia perennis Cantero et al. 1996

Sarcocornia ambigua-Heliotropium curassavicum

Lewis et Pire 1996

\section{8.- PROVINCIA PAMPEANA}

a.- de bosques, rodales diversos, etc.

Acacia caven-Geoffroea decorticans Lewis et al. $1985 \mathrm{c}$

Butia yatay Martínez Crovetto et Piccinini 1950

Carduus acanthoides-Cynodon dactylon Franceschi 1996

Celtis tala-Jodina rhombifolia Cabrera 1949

Celtis tala-Prosopis nigra Lewis et al. 1985c

Celtis tala-Jodina rhombifolia Faggi et Encabo 1987

Erythrina crista galli-Sapium haematospermum

Cabrera et Dawson 1944, Cabrera 1949

Erythrina crista galli-Zizaniopsis bonariensis Cabrera 1949

Nectandra falcifolia-Erythrina crista-galli Franceschi et al. 1985

Ocotea acutifolia-Allophyllus edulis Cabrera et Dawson 1944
Pouteria salicifolia-Allophylus edulis Cabrera et Dawson 1944

Prosopis caldenia-Condalia microphylla Cano et Movia 1967

Prosopis caldenia-Geoffroea decorticans Cano et Movia 1967

Prosopis caldenia-Larrea divaricata Cano et Movia 1967

Prosopis caldenia-Stipa tenuissima Cano et Movia 1967

Sebastiania brasiliensis-Lonchocarpus nitidus Cabrera et Dawson 1944

Tamarix gallica-Cynodon dactylon González Loyarte 1989b

Tessaria integrifolia-Salix humboltiana Franceschi et al. 1985

\section{b.- arbustivas diversas}

Baccharis salicifolia-Teucrium vesicarium Franceschi et al. 1985

Baccharis notosergila-Pterocaulon cordobense Cabrera 1949

Baccharis spartioides-Poa lanuginosa Cano et al. 1980

Baccharis tandilensis Frangi 1985

Baccharis tandilensis-Eupatorium buniifoliumBaccharis articulata Frangi 1985

Eupatorium buniifolium Frangi 1985

Eupatorium tweedianum-Hysterionica pinifolia Frangi 1985

Hibiscus cisplatinus-Polygonum hydropiperoides Franceschi et al. 1985

Hyalis argentea var. latisquama-Cenchrus pauciflorus Cano et Movia 1967

Lantana foetida-Pavonia xanthogloea Cabrera et Dawson 1944

Mimosa pigra-Spilanthes stolonifera Franceschi et al. 1985

Pavonia xanthogloea-Glandularia megapotamica Cabrera et Dawson 1944

Rubus ulmifolius-Cestrum parqui Cabrera et Dawson 1944

Verbena bonariensis-Aster squamatus Cabrera et Dawson 1944

c.- de pajonales, pastizales, praderas, etc.

Andropogon laguroides-Piptochaetium montevidensis Cabrera 1949

Bothriochloa springfieldii Cano et al. 1988a

Bromus catharticus-Heimia salicifolia Hilgert et D'Angelo 1996 


\section{E. Martínez Carretero et al. - Prodromus Sinsistemático y Estudios Fitosociológicos}

Cenchrus pauciflorus-Bromus brevis Cano et al. 1980

Cenchrus pauciflorus-Stipa tenuis Cano et Movia 1967

Cynodon dactylon-Alternanthera philoxeroides

Faggi et Encabo 1987

Cynodon dactylon-Ambrosia tenuifolia González Loyarte 1989b

Cynodon dactylon-Aristida adscencionis González Loyarte $1989 \mathrm{~b}$

Cynodon dactylon-Cirsium vulgare González Loyarte $1898 \mathrm{~b}$

Cynodon dactylon-Clematis denticulata González Loyarte 1989b

Distichlis spicata-Paspalum vaginatumHeliotropium curassavicum-M. cylindrica Burkart et al. 1990

Elionorus muticus-Hyalis argentea Cano et al. 1988a

Elionorus viridulus-Aristida mendocina Cano et Movia 1967

Eryngium elegans Frangi 1985

Eryngium paniculatum Frangi 1985

Eryngium stenophyllum-Blechnum australe var. auriculatum Frangi 1985

Melica brasiliana-Diodia dasycephala-Echium plantagineum Burkart et al. 1990

Melica macra-Phyla canescens Lewis et al. 1985c

Mentha pulegium-Leontodon taraxacoides-

Paspalidium paludivagum Burkart et al. 1990

Panicum decipiens-Paspalum vaginatum Cabrera 1949

Pappophorum caespitosum-Poa lanuginosa Cano et al. 1980

Pappophorum caespitosum Cano et al. 1988b

Paspalum quadrifarium Frangi 1985

Paspalum quadrifarium-Rumex crispus Lewis et al. $1985 \mathrm{c}$

Piptochaetium montevidense Frangi 1985

Piptochaetium montevidense-Ambrosia tenuifolia-E. bellidioides-Mentha pulegium Burkart et al. 1990

Poa ligularis-Piptochaetium napostaense Cano et al. 1988 a

Setaria parviflora-Verbena intermedia Hilgert et D'Angelo 1996

Sorghastrum pellitum Cano et al. 1988a

Sporobolus indicus-Hordeum stenostachys Lewis et al. 1984

Sporobolus pyramidatus-Chloris halophila Collantes et Lewis 1981

Stenotaphrum secundatum-Medicago lupulina Faggi et Cagnoni 1991
Stenotaphrum secundatum-Sporobolus poiretii Cabrera 1948, 1949

Stipa ambigua-Poa lanuginosa Cano et al. 1980

Stipa (Amelichloa) brachychaeta-Wedelia glauca Lewis et al. 1984

Stipa brachychaeta Cano et al. 1988b

Stipa hyalina-Carex bonariensis Collantes et Lewis 1981

Stipa hyalina-Sporobolus indicus Collantes et Lewis 1981

Stipa hyalina-Panicum milioides Hilgert et D'Angelo 1996

Stipa (Nassella) hyalina-Sporobolus indicus Lewis et al. 1984

Stipa (Nassella) hyalina-Carex bonariensis Lewis et al. 1984

Stipa (Nassella) neesiana-Bothriochloa laguroides Lewis et al. 1984

Stipa neesiana-Bromus catharticus Hilgert et D'Angelo 1996

Stipa papposa-Bothriochloa laguroides Collantes et Lewis 1981

Stipa papposa-Stipa formicarum Cabrera 1948, 1949

Stipa tenuis Cano et al. 1988b

Stipa tenuissima-Stipa ichu Cano et Movia 1967

Stipa tenuissima-Carduus nutans Cano et al. 1988a

d.- lacustres, palustres, de vegas, etc.

Alternanthera ficoides-Eragrostis hypnoides Fontana 1991b

Carex riparia-Malvella leprosa Lewis et al. 1985c

Celtetum Vervoorst 1967

Cortaderia selloana-Eryngium horridum Cabrera 1945

Cortaderia selloana Frangi 1985

Cyperus entrerianus Hilgert et D'Angelo 1996

Cyperus giganteus-C. surinamensis Faggi et Encabo 1987

Cyperus-Juncetum Vervoorst 1967

Distichletum Vervoorst 1967

Echinochloa helodes-Solanum glaucophyllum Lewis et al. 1985b

Echinochloa elodes-Senecio bonariensis Faggi et Encabo 1987

Echinochloa helodes Hilgert et D'Angelo 1996

Echinochloa polystachya-Polygonum acuminatum Franceschi et Prado 1989

Eichhornia crassipes-Salvinia herzogii Franceschi et al. 1985

Eichhornia crassipes Fontana 1991b 
Eleocharis bonariensis-Spilanthes stolonifera Cabrera et Dawson 1984

Eleocharis macrostachya-Aster squamatus Lewis et al. $1985 \mathrm{~b}$

Juncetum Vervoorst 1967

Juncus acutus var. leopoldii-Paspalum vaginatum Cabrera 1941

Juncus acutus-Picrosia longifolia Faggi et Cagnoni 1991

Lepidium spicata-Paspalum vaginatum Hilgert et D'Angelo 1996

Ludwigia peploides-Solanum glaucophyllum Franceschi et al. 1985

Ludwigia peploides-Enhydra anagallis Franceschi et Prado 1989

Ludwigia peploides-Polygonum punctatum Eskuche et Fontana 1996b

Luziola peruviana-Ludwigia peploides Lewis et al. 1985b

Panicum elephantipes-Eichhornia azurea Cabrera 1949

Panicum elephantipes-Polygonum ferrugineum Franceschi et al. 1985

Panicum elephantipes-Polygonum punctatum Franceschi et Prado 1989

Panicum decipiens-Paspalum vaginatum Cabrera 1948

Panicum prionitis-Oxalis sp. Franceschi et al. 1985

Panicum prionitis-Mikania periplocifolia Franceschi et Prado 1989

Panicum trichonaeloides Eskuche et Fontana 1996

Paspaletum Vervoorst 1967

Paspalum distichum-Paspalum vaginatum Lewis et al. $1985 \mathrm{~b}$

Polygonum acuminatum-Eleocharis elegans Franceschi et Prado 1989

Polygonum ferrugineum-Mikania sp. Franceschi et al. 1985

Polygonum hydropiperoides-Ambrosia elatior Franceschi et al. 1985

Polygonum stelligerum-Polygonum ferrugineum

Franceschi et al. 1985

Sagittaria montevidensis-Ludwigia peploides Lewis et al. 1985b

Sagittaria montevidensis-Pontederia rotundifolia Faggi et Encabo 1987

Sagittaria montevidensis Fontana 1991b

Scirpetum Vervoorst 1967

Scirpus californicus Frangi 1985

Scirpus chilensis-Eleocharis bonariensis Cabrera 1948
Scirpus giganteus-Solanum glaucum Cabrera 1948 Schoenoplectus californicus-Alternanthera philoxeroides Franceschi et al. 1985

Schoenoplectus californicus-Echinodorus grandiflorus Cabrera 1948

Schoenoplectus californicus-Polygonum punctatum Lewis et al. $1985 \mathrm{~b}$

Senecio bonariensis-Hydrocotyle bonariensis Franceschi et al. 1985

Solanetum Vervoorst 1967

Solanum glaucum-Glyceria fluitans Cabrera 1948

Solanum glaucum-Scirpus giganteus Cabrera 1949

Spartinetum Vervoorst 1967

Stipetum Vervoorst 1967

Stipo-Bothriochloetum Vervoorst 1967

Thalia geniculata-Polygonum acuminatum Franceschi et Prado 1989

Typha domingensis-Scirpus americanus Lewis et al. $1985 \mathrm{c}$

Typha latifolia Frangi 1985

Typha latifolia-Schoenoplectus californicus Faggi et Encabo 1987

Typhetum Vervoorst 1967

Victoria cruziana Fontana 1991b

Zizaniopsetum Vervoorst 1967

Zizaniopsis bonariensis-Scirpus giganteus Cabrera et Dawson 1984

Zizaniopsis bonariensis-Spilanthes stolonifera Cabrera et Dawson 1984

Zizaniopsis bonariensis-Schoenoplectus californicus Cabrera 1948

\section{e.- riparias (fluviales o marinas)}

Cakile maritima-Calycera crassifolia Faggi et Cagnoni 1991

Eryngium pandanifolium-Androtrichum trigynum Faggi et Cagnoni 1991

Spartinetum coarctatae Cabrera 1941, nom. inval., Eskuche 1973a

\section{f.- halófilas}

Agropyron scabrifolium-Hordeum stenostachys Lewis et al. $1985 \mathrm{c}$

Ambrosia tenuifolia Hilgert et D'Angelo 1996

Atriplex undulata-Distichlis scoparia Cano et al. 1980

Atriplex undulata-Distichlis spicata Cano et al. 1980 Atriplex undulata-Cressa truxillensis Cano et al. 1980 Atriplex undulata-Sarcocornia ambigua Verettoni 1961 


\section{E. Martínez Carretero et al. - Prodromus Sinsistemático y Estudios Fitosociológicos}

Cressa truxillensis-Sarcocornia ambigua Verettoni 1961

Distichlis spicata-Distichlis scoparia Cabrera 1945, 1948, 1949

Distichlis spicata-Paspalum vaginatum Collantes et Lewis 1980

Distichlis spicata-Scirpus americanus Verettoni 1961

Distichlis spicata-Paspalum vaginatum Lewis et al. 1985a

Distichlis spicata Hilgert et D'Angelo 1996

Sarcocornia ambigua-Heterostachys ritteriana Verettoni 1961, em.

Scirpus americanus-Paspalum vaginatum Lewis et al. $1985 \mathrm{a}$

Spartina argentinensis-Spartina densiflora Collantes et Lewis 1980

Spartina argentinensis-Paspalum vaginatum Lewis et al. $1985 \mathrm{a}$

Spartina argentinensis Stofella 1995

Spartina densiflora-Sarcocornia ambigua Lewis et al. $1985 \mathrm{a}$

Spartina densiflora-Limonium brasiliense Faggi et Cagnoni 1991

Sporobolus pyramidatus-Distichlis spicata Lewis et al. 1985 a

Suaeda patagonica-Frankenia microphylla Verettoni 1961

Verbena gracilescens Hilgert et D’Angelo 1996

\section{g.- psamófilas}

Adesmia incana-Medicago minima Cabrera 1940

Adesmia incana-Poa lanuginosa Cabrera 1941

Androtrichum trigynum-Tessaria absinthioides

Cabrera 1941

Hyalis argentea var. latisquama-Panicum urvilleanum Cabrera 1945

Panicum racemosum-Hydrocotyle bonariensis

Cabrera 1941

Panicum urvilleanum-Hyalis argentea var. latisquama Cabrera 1941

Scirpus olneyi-Paspalum vaginatum Collantes et Lewis 1981

Spartina ciliata-Calycera crassifolia Cabrera 1941

Spartina ciliata-Polygala cyparissias Faggi et Cagnoni 1991

Sporobolus rigens-Hyalis argentea var. latisquama Cabrera 1941

\section{9.- PROVINCIA DEL ESPINAL}

a.- de bosques, rodales diversos, etc.

Acacia-Prosopis Ragonese 1941

Acacia aroma-Acacia atramentaria (sabanas) D'Angelo et al. 1987

Andropogon lateralis-Axonopus affinis Carnevali 1994

Aspidosperma quebracho-blanco-Thrithrinax campestris-Prosopis spp. D'Angelo et al. 1987

Aspidosperma quebracho-blanco Carnevali 1994

Axonopus compressus-Paspalum notatum Carnevali 1994

Butia yatay Ragonese 1941

Celtis tala Faggi 1986

Celtis tala-Geoffroea decorticans Lewis et al. 2006

Celtis tala-Prosopis alba Lewis et al. 2009

Copernicia alba Ragonese 1941

Erythrina crista-galli Ragonese 1941

Geoffroea decorticans-Prosopis nigra D'Angelo et al. 1987

Paspalum durifolium Carnevali 1994

Phytolaca dioica Lewis et al. 1976

Prosopis affinis Carnevali 1994

Prosopis caldenia-Stipa ichu Cano et Movia 1967

Prosopis caldenia-Geoffroea decorticans Cano et Movia 1967

Prosopis caldenia-Jodina rhombifolia Cano et Movia 1967, Cano et al. 1980

Prosopis caldenia-Condalia microphylla Cano et Movia 1967

Prosopis caldenia-Stipa ambigua Cano et al. 1980

Prosopis spp.-Geoffroea decorticans (sabanas) D'Angelo et al. 1987

Prosopis nigra Ragonese 1941

Prosopis-Cereus coryne Ragonese 1941

Rhynchospora corymbosa var. asperula Carnevali 1994

Schinopsis balansae Ragonese 1941

Schinopsis balansae-Schinopsis lorentziiAspidosperma quebracho-blanco Ragonese 1941

Tessarietum Lewis et al. 1976

Thrithrinax campestris-Celtis tala Lewis et al. 2009

\section{b.-arbustivas diversas}

Baccharis salicifolia Lewis et al. 1976

Chuquiraga erinacea-Atriplex lampa Cano et al. 1980 
Larrea divaricata-Prosopis sp. Cano et al. 1980

Larrea cuneifolia Zabalza, Barreix et Cano 1989

Larrea nitida Zabalza, Barreix et Cano 1989

Senecio subulatus var. erectus-Cenchrus pauciflorus

Cano et Movia 1967

Polygonum portoricense Lewis et al. 1976

Sesbania virgata Lewis et al. 1976

Tessaria dodoneifolia-Baccharis lanceolata Ragonese 1941

Tessaria dodoneifolia-Elionurus muticus (sabanas)

D'Angelo et al. 1987

c.- de pajonales, pastizales, praderas, etc.

Andropogon lateralis Carnevali 1994

Andropogoneto-Axonopetum Martínez Crovetto 1965 , nom. inval.

Aristida jubata Carnevali 1994

Aristida spegazzinii Zabalza, Barreix et Cano 1989

Aristideto-Paspaletum Martínez Crovetto 1969, nom. inval.

Axonopo-Paspaletum Martínez Crovetto 1969, nom. inval.

Bothriochloa springfieldii Zabalza, Barreix et Cano 1989

Echinochloa helodes Faggi 1986

Eleocharis viridans Faggi 1986

Eleocharis bonariensis Faggi 1986

Elionorus Ragonese 1941

Elionurus muticus-Cynodon dactylon D'Angelo et al. 1987

Elionorus muticus Carnevali 1994

Hyalis argentea Zabalza, Barreix et Cano 1989

Hyalis argentea-Elyonorus muticus Cano 1990

Leptochloa chloridiformis Ragonese 1941

Melicetum Lewis et al. 1976

Myriophyllum aquaticum Faggi 1986

Nassella neesiana-Setaria geniculata D'Angelo et al. 1987

Panicum prionitis Ragonese 1941

Paspalum-Axonopus Ragonese 1941

Paspalum notatum Carnevali 1994

Pennisetum frutescens (poblaciones puras) D'Angelo et al. 1987

Piptochetium napostaense-Stipa tenuis Cano 1990

Poa ligularis Zabalza, Barreix et Cano 1989

Scirpus californicus Faggi 1986

Selaginello-Ayenietum o'donellii Martínez Crovetto 1980a, nom. inval.

Sorghastrum nutans-Axonopus suffultus D'Angelo et al. 1987
Sorghastrum nutans-Paspalum brunneum Carnevali 1994

Stipa gynerioides Zabalza, Barreix et Cano 1989

Stipa tenuissima-Stipa ichu Cano et Movia 1967

Stipo-Bothriochloetum Lewis et al. 1976

Stipo-Caricetum Lewis et al. 1976

Stipo-Sporoboletum Lewis et al. 1976

\section{d.- lacustres, palustres, de vegas, etc.}

Cyperus entrerianus (poblaciones puras) D'Angelo et al. 1987

Cyperus giganteus Ragonese 1941

Echinochloa helodes-Paspalidium paludivagum

D'Angelo et al. 1987

Echinochloetum Lewis et al. 1976

Eichhornietum solanetosum Lewis et al. 1976

Eleocharis macrostachya-Eleocharis elegans

D'Angelo et al. 1987

Eugenia cisplatensis Ragonese 1941

Hemarthria altissima Ragonese 1941

Luziola peruviana-Leersia hexandra D'Angelo et al. 1987

Paspalum distichum-Cyperus digitatus D'Angelo et al. 1987

Paspaletum distichi Lewis et al. 1976

Paspaletum quadrifarii Lewis et al. 1976

Sagittaria montevidensis-Ludwigia peploides

D'Angelo et al. 1987

Scirpus americanus (poblaciones puras) D'Angelo et al. 1987

Scirpus robustus (poblaciones puras) D'Angelo et al. 1987

Scirpo-Paspaletum Lewis et al. 1976

Schoenoplectus californicus-Ludwigia peploides

D'Angelo et al. 1987

Spartinetum argentinensis Lewis et al. 1976

Spartinetum densiflora Lewis et al. 1976

Thalia multiflora (poblaciones puras) D'Angelo et al. 1987

Typha domingensis (poblaciones puras) D'Angelo et al. 1987

\section{e.- halófilas}

Atriplex undulata-Heterostachys ritteriana Cano et al. 1980

Atriplex undulata-Sesuvium portulacastrum Cano et al. 1980

Baccharis spartioides-Atriplex undulata Cano et al. 1980

Cyclolepis genistoides-Allenrolfea patagonica Cano et al. 1980 


\section{E. Martínez Carretero et al. - Prodromus Sinsistemático y Estudios Fitosociológicos}

Cyclolepis genistoides-Heterostachys ritteriana Cano et al. 1980

Cyclolepis genistoides-Lycium chilense Cano et al. 1980

Distichlis spicata-Distichlis scoparia Cano et al. 1980

Distichlis spicata-Suaeda patagonica Cano et al. 1980

Distichilis spicata-Cynodon dactylon D'Angelo et al. 1987

Distichlis spicata Cano 1990

Juncus acutus Faggi 1986

Heterostachys ritteriana-Sarcocornia ambigua D'Angelo et al. 1987

Leptochloa chloridiformis-Cynodon dactylon D'Angelo et al. 1987

Oxalis mallabolba Faggi 1986

Paspalum vaginatum (praderas saladas) D'Angelo et al. 1987

Salicornia-Distichlis spicata Ragonese 1941

Sarcocornia ambigua-Distichlis spicata Cano et al. 1980

Sarcocornia ambigua-Polygonum elongatum Cano et al. $1980 \mathrm{em}$.

Sarcocornia ambigua-Tamarix gallica Cano et al. 1980

Solanum elaeagnifolium-Hordeum stenostachys Cano et al. 1980

Solanum elaeagnifolium-Stipa papposa Cano et al. 1980

Spartina argentinensis Ragonese 1941

Spartina argentinensis-Hordeum stenostachys D'Angelo et al. 1987

Spartina densiflora-Sarcocornia ambigua D'Angelo et al. 1987

Spergularia marina Faggi 1986

Sporobolus pyramidatus-Distichlis spicata D'Angelo et al. 1987

\section{f.- psamófilas}

Andropogono-Centelletum Martínez Crovetto 1962, nom. inval.

Axonopo-Cyperetum Martínez Crovetto 1962, nom. inval.

Cynodono-Daucetum Martínez Crovetto 1962, nom. inval

Elionurus viridulus-Hyalis argentea var. latisquama Cano et Movia 1967

Elionuro-Panicetum Martínez Crovetto 1962, nom. inval.

Hyalis argentea var. latisquama-Cenchrus pauciflorus Cano et Movia 1967

Hyalis argentea var. latisquama-Stipa tenuissima Cano et Movia 1967

Limnanthemo-Cyperetum Martínez Crovetto 1962, nom. inval.

Paspalum dilatatum-Cynodon dactylon Faggi 1986

\section{0.- PROVINCIA DEL MONTE}

\section{a.- de bosques, rodales diversos, etc.}

Acacia furcatispina-Cercidium praecox subsp. glaucum Bertolami 1990

Atriplex lampa-Larrea cuneifolia Peralta 1994

Bulnesia retama Méndez, Martínez Carretero et Wuilloud 1993

Bulnesia retama-Larrea divaricata Tanquilevich 1971

Bulnesia retama-Lycium tenuispinosum Roig 1970

Bulnesia retama-Tephrocactus aoracanthus Roig 1973b

Geoffroea decorticans-Atriplex lampa Tanquilevich 1971

Geoffroea decorticans-Condalia microphylla Roig 1970

Geoffroea decorticans Méndez, Martínez Carretero et Wuilloud 1993

Geoffroea decorticans-Prosopis alpataco Villagra et Roig 1999

Prosopis flexuosa var. flexuosa Méndez, Martínez Carretero et Wuilloud 1993

Prosopis alba-Lycium tenuispinosum Roig et Paladini 1994

Prosopis flexuosa-Atriplex lampa Roig 1970, Therburg 1997

Prosopis flexuosa-Larrea divaricata Tanquilevich 1971, Villagra et Roig 1999

Prosopis flexuosa-Suaeda divaricata González Loyarte et al. 2000

Prosopis flexuosa-Trichomaria usillo González Loyarte et al. 2000

Proustia cuneifolia-Baccharis retamoides Roig 1973b

\section{b.- arbustivas diversas}

Adesmia trijuga-Tricycla spinosa Roig 1973b

Artemisia mendozana Martínez Carretero 1985

Artemisia mendozana-Erioneuron pilosum Martínez Carretero 1999

Asarina antirrhiniflora-Cynodon dactylon Martínez Carretero et Roig 1994

Atriplex lampa-Aristida mendocina Tanquilevich 1971 
Atriplex lampa-Pappophorum caespitosum Roig 1970

Atriplex lampa Méndez, Martínez Carretero et Wuilloud 1993

Atriplex lampa-Junellia seriphioides De Marco et al. 1993

Atriplex lampa-Larrea nitida De Marco et al. 1993

Baccharis salicifolia Martínez Carretero et Dalmasso 1995

Cercidium praecox subsp. glaucum-Hyalis argentea var. latisquama Martínez Carretero 1999

Colliguaja integerrima Martínez Carretero 1984

Colliguaja integerrima-Baccharis petiolata Martínez Carretero 1985

Cortesia cuneifolia Martínez Carretero et Dalmasso 1995

Chuquiraga erinacea-Hyalis argentea Martínez Carretero et Dalmasso 1995

Deuterochnia longisepala-Opuntia sulphurea Roig 1976

Dolychlasium lagascae-Tricycla spinosa Martínez Carretero 1999

Eupatorium buniifolium Martínez Carretero 1985

Eupatorium buniifolium-Tessaria dodoneifolia Martínez Carretero 1999

Fabiana denudata Méndez, Martínez Carretero et Wuilloud 1993

Gochnatia glutinosa-Lycium tenuispinosum Bertolami 1990

Gochnatia glutinosa-Zuccagnia punctata Roig 1976

Larrea cuneifolia-Scleropogon brevifolius Roig 1970

Larrea cuneifolia-Trichloris crinita Roig 1970

Larrea cuneifolia-Atriplex lampa Ambrosetti et al. 1986

Larrea cuneifolia-Atriplex spegazzinii Ambrosetti et al. 1986

Larrea cuneifolia-Pappophorum caespitosum González Loyarte 1989b

Larrea cuneifolia-Lycium tenuispinosum Tanquilevich 1971, Bertolami 1990

Larrea cuneifolia-Monttea aphylla De Marco et al. 1993

Larrea cuneifolia-Trichocereus candicans Peralta 1994

Larrea cuneifolia Martínez Carretero et Dalmasso 1995

Larrea cuneifolia Dalmasso et Márquez 2004

Larrea divaricata Martínez Carretero 1984

Larrea divaricata-Junellia aspera Ambrosetti et al. 1986
Larrea divaricata-Junellia ligustrina Böcher et al. 1972

Larrea divaricata-Junellia aspera Roig 1976

Larrea divaricata-Schinus fasciculata Roig 1976

Larrea divaricata-Monttea aphylla Ambrosetti et al. 1986

Larrea divaricata-Fabiana peckii Roig Juñent 1989

Larrea divaricata-Acantholippia seriphioides Martínez Carretero 1985, 1987

Larrea divaricata-Pappophorum philippianum González Loyarte 1989b

Larrea divaricata-Acantholippia seriphioides Martínez Carretero et Roig 1992

Larrea divaricata Méndez, Martínez Carretero et Wuilloud 1993

Larrea divaricata-Acantholippia seriphioides Peralta 1994

Larrea divaricata Martínez Carretero et Dalmasso 1995

Larrea divaricata-Junellia aspera Roig et Martínez Carretero 1992

Larrea divaricata-Prosopis flexuosa var. depressa Roig et Martínez Carretero 1992

Larrea divaricata-Stipa tenuis De Marco et al. 1993 Larrea divaricata-Junelia seriphioides De Marco et al. 1993

Larrea divaricata-Fabiana denudata Martínez Carretero 2000

Larrea divaricata-Lycium tenuispinosum Martínez Carretero 2000

Larrea divaricata-Nicotiana noctiflora Martínez Carretero 2000

Larrea divaricata Dalmasso et Márquez 2004

Lycium teuispinosum-Pappophorum cespitosum González Loyarte 1989b

Prosopidastrum globosum Méndez, Martínez Carretero et Wuilloud 1993

Prosopis alpataco-Capparis atamisquea Villagra et Roig 1999

Senna aphylla-Acantholippia seriphioides Roig 1971

Senecio subulatus Méndez, Martínez Carretero et Wuilloud 1993

Schinus fasciculata-Piptochaetium napostaense De Marco et al. 1993

Verbena seriphioides Méndez, Martínez Carretero et Wuilloud 1993

Zuccagnia punctata Martínez Carretero 1985

Zuccagnia punctata-Pappophorum caespitosum Bertolami 1990 


\section{E. Martínez Carretero et al. - Prodromus Sinsistemático y Estudios Fitosociológicos}

Zuccagnia punctata-Artemisia mendozana Roig 1976

c.- de pajonales, pastizales, praderas, etc.

Aristida inversa Méndez, Martínez Carretero et Wuilloud 1993

Hyalis argentea var. latisquama Méndez, Martínez Carretero et Wuilloud 1993

Muhlenbergia torreyi-Poa lanuginosa De Marco et al. 1993

Stipa chrysophylla var. crispula-Stipa tenuis De Marco et al. 1993

Stipa chrysophylla var. crispula-Larrea nitida De Marco et al. 1993

Stipa eriostachya Martínez Carretero 1984

Stipa ichu-Acantholippia seriphioides Roig Juñent 1989

Stipa ichu-Eragrostis lugens Martínez Carretero 1985

Stipa ichu-Fabiana peckii Roig 1976

Stipa ichu-Lippia turbinata Martínez Carretero 1987

Stipa scirpea-Porophyllum lanceolatum Martínez Carretero 1987

Stipa tenuissima Martínez Carretero 1984

Stipa tenuissima-Verbascum thapsus Martínez Carretero 1985

Stipa tenuissima-Stipa tenuis De Marco et al. 1993

d.- lacustres, palustres, de vegas, etc.

Acacia furcatispina-Baccharis retamoides Bertolami 1990

Baccharis salicifolia-Tamarix gallica Villagra et Roig 1999

Baccharis spartioides-Cerastium arvense Martínez Carretero 1987

Clematis montevidensis-Eupatorium patens Tanquilevich 1971

Cortaderia rudiuscula-Baccharis absinthioides Villagra et Roig 1999

Eupatorium patens-Baccharis angulata Roig 1970

Eupatorium patens-Salvia gilliesii Martínez Carretero 1987

Lemma minuscula-Salvinia minima Eskuche 1986

Sporobolus indicus-Glyzyrrhiza astragalina Villagra et Roig 1999

Wolffiella oblonga-Lemma gibba Landolt 1986

\section{e.- riparias}

Argemone subfusiformis Martínez Carretero 1985

Cortaderia rudiuscula Martínez Carretero 1985
Senecio gilliesianum-Pappophorum caespitosum Martínez Carretero 1987

\section{f.-halófilas}

Allenrolfea vaginata-Prosopis strombulifera Therburg 1997

Atriplex argentina-Suaeda divaricata Therburg 1997

Atriplex lampa-Grahamia bracteata Therburg 1997

Atriplex spegazzinii-Grahamia bracteata Therburg 1997

Suaeda divaricata-Distichlis spicata Martínez Carretero 2000

Suaeda divaricata-Prosopis strombulifera Therburg 1997

\section{g.- psamófilas}

Adesmia trijuga-Senecio pinnatus Méndez 1992

Calycera spinulosa-Parthenium hysterophorus De Marco et al. 1993

Ephedra boelckei-Larrea divaricata Roig 1985

Gomphrena martiana-Heliotropium mendocinum Hueck 1950

Hyalis argentea var. argentea-Atriplex deserticola Martínez Carretero 2000

Hyalis argentea var. latisquama-Cercidium praecox subsp. glaucum De Marco et al. 1993

Larrea divaricata-Panicum urvilleanum Roig 1970

Neosparton aphyllum-Panicum urvilleanum Méndez 1992

Neoparton aphyllum-Stipa neaei Martínez Carretero 2000

Sporobolus rigens Hueck 1950

h.- saxícolas

Adesmia trijuga-Prosopidastrum globosum De Marco et al. 1993

Astericium glaucum-Tillandsia xyphioides Martínez Carretero 1987

Blossfeldia liliputana-Plagiochasma aff. rupestre Méndez 1983

Cercidium praecox Martínez Carretero 1985

Chuquiraga erinacea-Pappophorum caespitosum Bertolami 1990

Dolychlasium lagascae Martínez Carretero 1985

Mentzelia parvifolia-Schinus fasciculata Martínez Carretero 1987 
Stipa psittacorum-Chuquiraga erinacea Martínez Carretero 1987

i.- adventicias, ruderales, etc.

Sonchus arvensis-Cynodon dactylon Roig 1994

IV.- Territorio de las selvas subtropicales

(Provincias Paranense y de las Yungas)

\section{1.- PROVINCIA PARANENSE}

a.- de bosques, rodales diversos, etc.

Albizia inundata-Inga affinis Fontana 2015

Anadenanthera cebil Fontana 2015

Astronium balansae Fontana 2015

Balfourodendron riedelianum Fontana 2015

Cathormion polyanthum-Inga uruguensis Eskuche 1984, 1986

Celtis tala Chichizola 1993

Cordia americana Fontana 2015

Erythrina crista-galli Kandus et al. 2003

Handroanthus heptaphylla Fontana 2015

Helietta apiculata-Astronium balansae Eskuche 1986

Nectandra falcifolia Franceschi et al. 1985

Nectandra megapotamica Fontana 2016

Parapiptadenia rigida-Nectandra saligna Eskuche 1984, 1986

Parapiptadenia rigida-Nectandra salignaAspidosperma polyneuron Eskuche 1984, 1896

Salix humboldtiana Franceschi et al. 1985

Schinopsis balansae-Astronium balansaeBrunfelsia australis Eskuche 1986

Tessaria integrifolia Franceschi et al. 1985

Trema micrantha-Solanum granuloso-leprosum Eskuche 1986

Holocalyx balansae-Ficus luschnathiana Prado et al. 1989.

\section{b.- arbustivas diversas}

Acacia caven Franceschi et al. 1985

Baccharis salicifolia Franceschi et al. 1985

Bromelia serra-Aechmea disticantha Martínez Crovetto 1980 b

Hibiscus cisplatinus Franceschi et al. 1985

Menodora trifida-Selaginella sellowii Eskuche 1986

Mimosa pigra Franceschi et al. 1985

Solanum amygdalifolium-Cissus palmata Eskuche 1986

Turnera grandiflora-Elymus muticus Eskuche 1986 c.- de pajonales, pastizales, praderas, etc.

Andropogon lateralis Parodi 1943

Andropogon lateralis-Axonopus compressus

Martínez Crovetto 1965

Andropogon lateralis Fontana 2016

Andropogon virgatus Fontana 2015

Aristida jubata Fontana 2015

Axonopodo affinis-Paspaletum notati Fontana 1996 Axonopus fissifolius-Paspalum notatum Eskuche 1986

Eichhornia crassipes-Salvinia herzogii Franceschi et al. 1985

Elionurus muticus-Calea uniflora Eskuche1979

Elionurus muticus-Cnidosculus albomaculatus

Vallejos 1993

Elionorus muticus Fontana 2015

Elionurus viridulus Parodi 1943

Fraileo-Cheilanthetum micropteridis Fontana 1996

Leersia hexandra-Luziola peruviana Vallejos 1993

Ludwigia peploides Franceschi et al. 1985

Ludwigia peploides-Polygonum punctatum

Eskuche et Fontana 1996

Panicum elephantipes Franceschi et al. 1985

Panicum gynerioides Parodi 1943

Panicum grumosum Kandus et al. 2003

Panicum prionitis Franceschi et al. 1985

Panicum prionitis Fontana 2015

Panicum tricholaenoides Eskuche et Fontana 1996b

Paspalum guaraniticum Parodi 1943

Paspalum notatum-Axonopus fisifolius Fontana 2015

Polygono punctati-Rumicetum paraguariensis Fontana 1991 (Eskuche 2004)

Rhynchospora tenuis-confinis Eskuche 1986

Salvia nervosa-Elionurus muticus-Butia yatay Eskuche 1986

Schoenoplectus californicus Chichizola 1993

Senecio bonariensis Franceschi et al. 1985

Scirpus americanus ssp. monophyllus Chichizola 1993

Scirpus californicus Franceschi et al. 1985

Scirpus giganteus Chichizola 1993

Sorghastrum agrostoides-Paspalum notatum Vallejos 1993

Sorghastrum pellitum-Eryngium eburneum Fontana 2015

Spartina densiflora Chichizola 1993

Zornio orbiculatae-Trigopogonetum spicati Fontana 1996

Waltheria detonsa-Polycarpea brasiliensis Eskuche 1979 


\section{E. Martínez Carretero et al. - Prodromus Sinsistemático y Estudios Fitosociológicos}

\section{Selaginelletum sellowii Fontana 1996}

d.- lacustres, palustres, vegas, riparia, etc. Apinagia yguazuensis Fontana 2014 Azolla filiculoides-Pistia stratiotes Chichizola 1993

Bacopa salzmannii-Mayaca fluviatilis Eskuche 1986

Cecropia pachystachya Fontana 2015

Croton urucurana Fontana 2015

Cyperus giganteus Fontana 2015

Eichhornia crassipes Fontana 2015

Fuirena robusta Fontana 2015

Lemma crassipes-Salvinia minima Eskuche 1986

Lindernio-Mercadonietum hernarioidis Eskuche 1975

(Bidendetetea tripartitae vel Isoeto-

Nanojuncetea?)

Mayaca sellowiana Fontana 2015

Mourera aspera Fontana 2014

Podostemum atrichum Fontana 2014

Podostemum comatum Fontana 2014

Podostemum muelleri Fontana 2014

Podostemum rutifolium Fontana 2014

Podostemum uruguayense Fontana 2014

Podostemetum undulati Drehwald 1990 (Fig. 11e)

Polygonum hispidum Kandus et al. 2003

Reussia rotundifolia-Eichhornia crassipes

Eskuche 1986

Schoenoplectus californicus Kandus et al. 2003

Scirpus cubensis-Pistia stratiotes Eskuche 1986

Scirpus giganteus Kandus et al. 2003

Sphagnum sp. Fontana 2015

Thalia geniculata Fontana 2015

Typha ssp.-Cyperus giganteus Kandus et al. 2003

Wolffiela lingulata-Salvinia minima Eskuche et

Romero Fonseca 1982

Wolffiela lingulata-Pistia stratiotes Eskuche et Romero Fonseca 1982

Wolffiella lingulata-Spirodela intermedia Eskuche et Romero Fonseca 1982

Wolffiella lingulata-Salvinia auriculata Eskuche et Romero Fonseca 1982

Zizaniopsis bonariensis Kandus et al. 2003

\section{2.- PROVINCIA DE LAS YUNGAS}

a.- de bosques, rodales diversos, etc. Alnus jorullensis Asociación Hueck 1954 Alnus acuminata-Rapanea laetevirens Aceñolaza 1996
Alnus acuminata-Podocarpus parlatorei Aceñolaza 1996

Alnus acuminata-Duranta serratifolia Aceñolaza 1996

Alnus acuminata-Podocarpus parlatorei Martínez et Prado 2013

Anadenanthera colubrina var. cebilParapiptadenia excelsa Martínez et Prado 2013

Calycophyllum multiflorum-Phyllostylon rhamnoides Prado 1995

Cinnamomum porphyrium-Juglans australis Martínez et Prado 2013

Phoebe porphyria-Blepharocalyx gigantea Eskuche 1984, 1986

Schino bumeloidis-Allophyletum edulis Entrocassi 2015

Tecomo stantis-Anaderantheretum cebilis Entrocassi 2015

Tipuana tipu-Enterolobium contortisiliquum Prado 1995

b.- de pajonales, pastizales, praderas, etc

Argyrochosma nivea-Campyloneurum angustipaleatum (de helechos de Pastizal de Altura) Martínez et Prado 2013

Cortaderia (=Lamprothyrsus) hieronymi-Jarava pseudo-ichu Martínez et Prado 2013

Jarava ichu-Piptochaetium montevidense Martínez et Prado 2013

c.- de helechos del sotobosque de Yungas

Megalastrum pulverulentum-Ophioglossum reticulatum (Selva Montana) Martínez et Prado 2013

Pellaea ovata-Anogramma lorentzii (Selva Pedemontana) Martínez et Prado 2013

\section{Diversidad de las unidades sintaxonómicas}

Considerando la información florística reunida en los 195 relevamientos fitosociológicos que representan a las clases de vegetación, resulta que en promedio los Piptochaetio-Stipetea neesianae y Elyonuretea mutici (Chaco) poseen 33 y 21 especies, respectivamente. Le siguen Chiliotrichetea diffusii y Festucetea gracillimae (Patagonia) con 23 y 20 especies y Empetro bolacetea (Subantarctica) con 20 especies, evidenciando los sectores bioclimáticamente más húmedos de Argentina. Por otra parte, Dolychlasietea lagascae (Cardonal) con 5 especies y Calamagrostietea vicunarum (Puna) con 5 especies representan ambientes más secos (Fig. 8). 
Bol. Soc. Argent. Bot. 51 (3) 2016

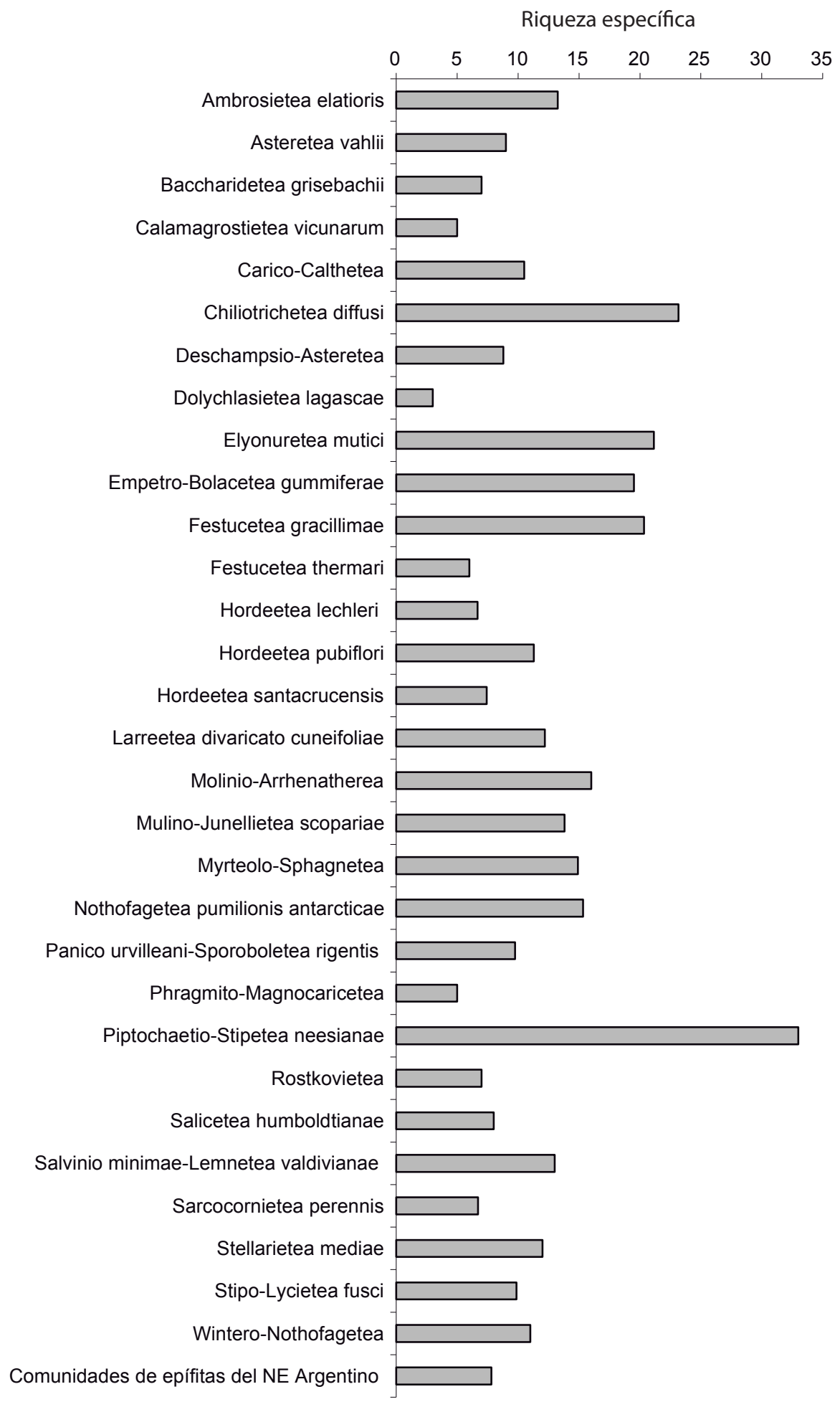

Fig. 8. Riqueza específica por clase de vegetación. 
E. Martínez Carretero et al. - Prodromus Sinsistemático y Estudios Fitosociológicos
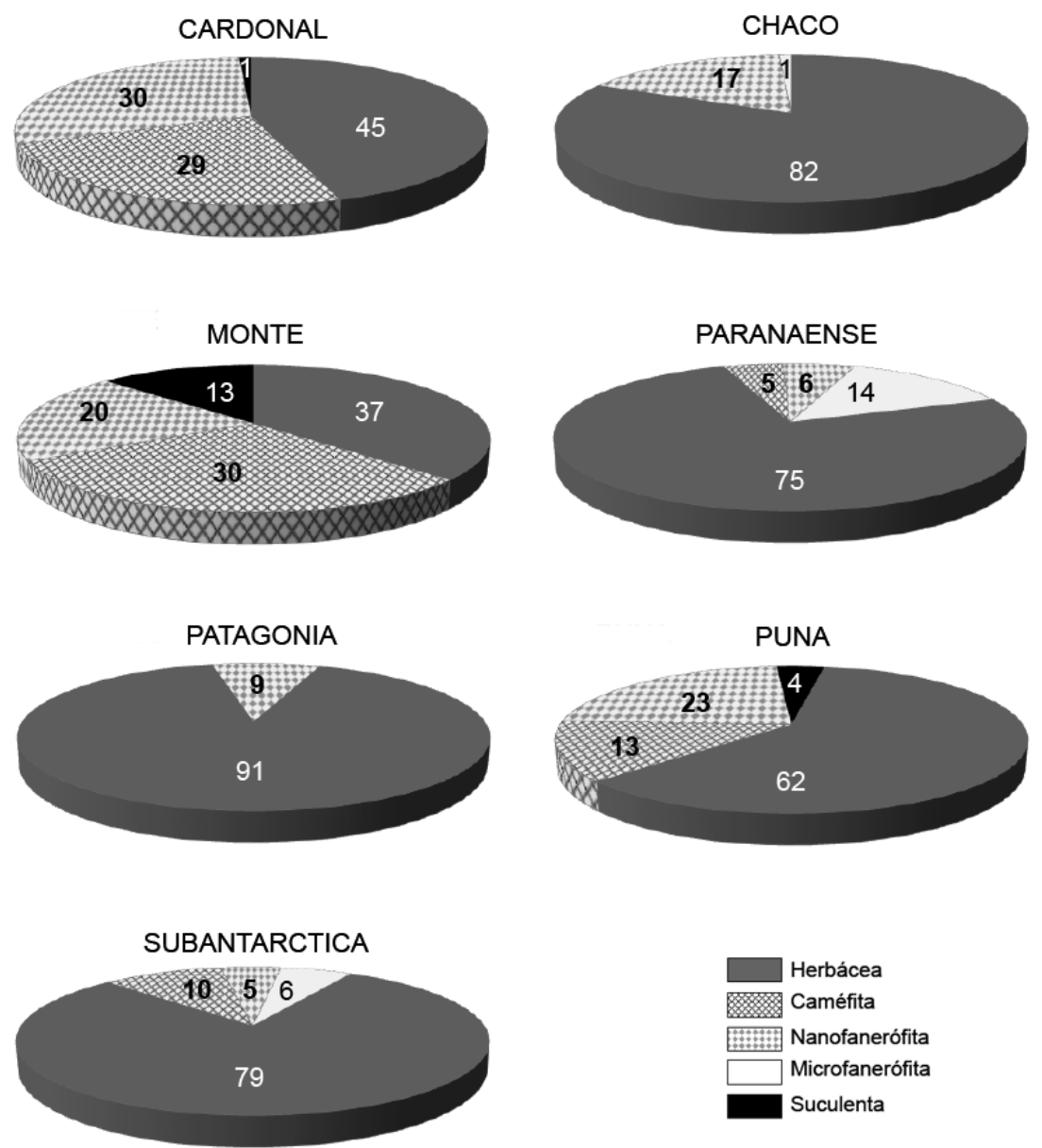

Fig. 9. Formas biológicas en porcentaje por provincia fitogeográfica. Números: porcentaje de presencia. 
Bol. Soc. Argent. Bot. 51 (3) 2016


Fig. 10. PUNA: a- Chuquirago-Lycietum fusci, b- Stipetum scirpeae; MONTE: c- Suaedo sarcocornietum, d- Larreetum cuneifoliae; ALTOANDINO: e- Oxychloetum andinae; SUBANTARCTICA: f- AdesmioAraucarietum, g- Pernettyo mucronatae-Nothofagetum pumilionis; PAYUNIA: h- Neospartetum aphylly, i- Stipetum chrysophyllae crispulae; CARDONAL: j- Dolychlasietum lagascae. 


\section{E. Martínez Carretero et al. - Prodromus Sinsistemático y Estudios Fitosociológicos}
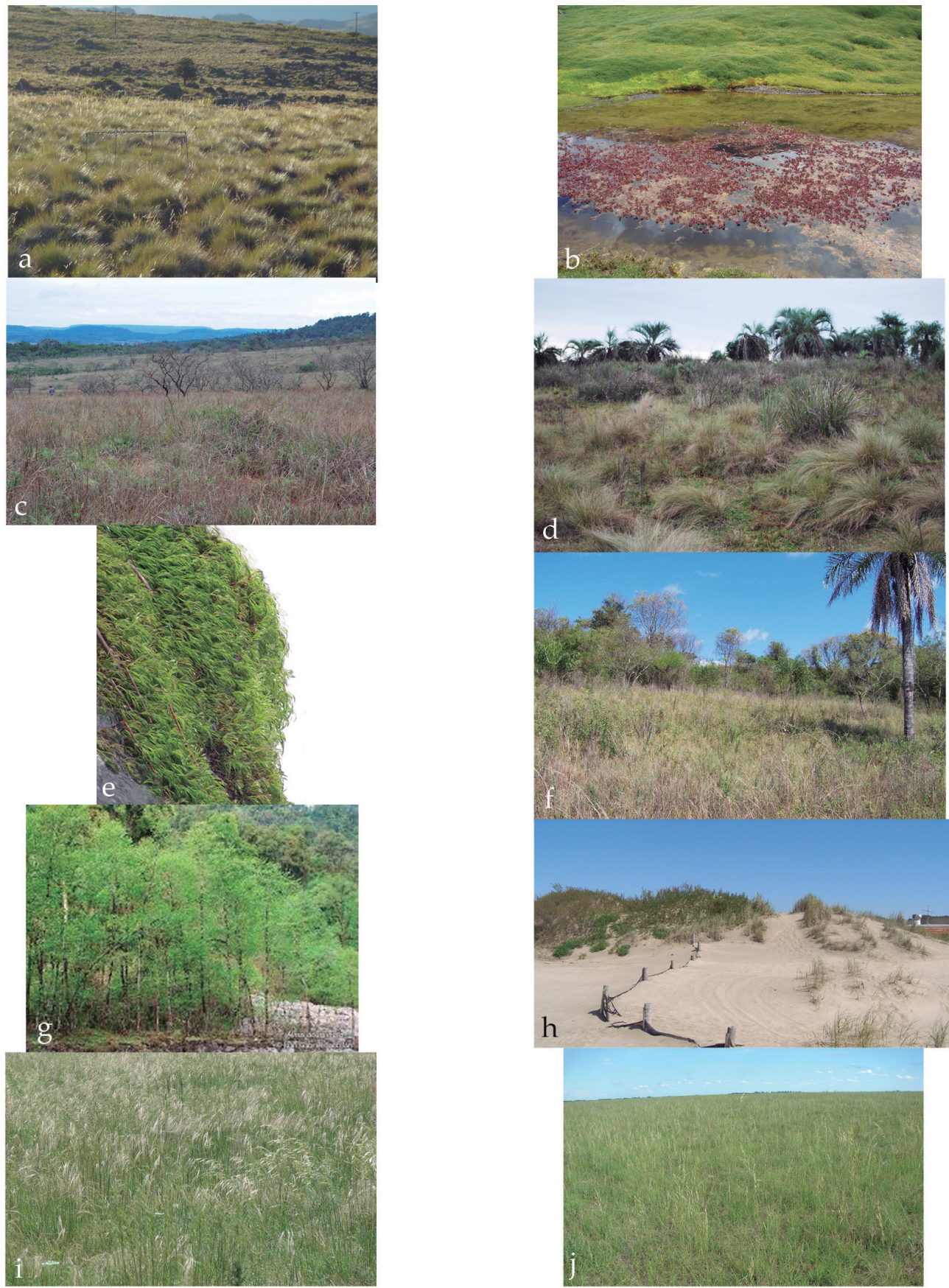

Fig. 11. a- PATAGONIA: Festucetum gracillimae, b- Hippuridetum vulgaris; PARANAENSE: c- Diplothemio campestris- Axonopodetum suffulti, d- Macrosiphonio undulatae-Aristidetum jubatae, e- Podostemetum undulati; CHACO: f- Stevio entrerriensis-Elyonurion mutici; YUNGAS: g- Polysticho montevidensis-Alnetum acuminatae; PAMPEANA: h- Panicetum racemosi; ESPINAL: i- Stipetum neesianae, j- Schlechtendalio luzulaefoliae-Brometum auletici. 
Teniendo en cuenta la estructura a través de las formas biológicas, las microfanerófitas dominan en las clases de la región Paranaense, mientras que las nanofanerófitas, en general arbustivas altas, en las del Cardonal, Monte y Puna. Las caméfitas, arbustivas bajas, lo hacen en las de Patagonia, Puna, Cardonal y Monte. Las suculentas se restringen a la vegetación del Monte, Puna y Cardonal (Fig. 9). Las herbáceas están presentes con elevada constancia en todas las unidades de vegetación. Se evidencia el gradiente higrotérmico creciente en sentido este-oeste, así como la Diagonal Árida Argentina asociada al sistema andino, con desiertos fríos en altura y cálidos al pie del borde oriental de la cordillera.

\section{Bibliografía}

ACEÑOLAZA, P. 1996. Fitosociología de los bosques de Aliso (Alnus acuminata) H.B.K. en la sierra de San Javier Tucumán Argentina. Doc. phytosociol., nouv. sér: : 505-516.

ADAMOLI, J., R. NEUMANN, A.D. RATIER DE COLINA \& J. MORELLO. 1972. El Chaco aluvional salteño. R.I.A. Serie 3, Clima y Suelo 10 (5): 165-237.

ALBOFF, N. 1896. Contributions a la flore de la Terre de Feu. I. Observations sur la végétation du Canal de Beag1e. Revista Mus. La Plata 7: 277-308.

ALFONSO, J.L. 1940. Los bosques de Tierra de Fuego. Bosques y Obrajes 6. Buenos Aires.

AMBROSETTI, A., E. MÉNDEZ \& F. ROIG. 1968. Observaciones sobre una comunidad vegetal clausurada a los animales pero modificada por el fuego en el Oeste de la Provincia de Mendoza y su importancia en el control biológico de la erosión torrencial y las pasturas. Revista Fac. Cienc. Agr. 14: 3-26.

AMBROSETTI, A., L. DEL VITTO \& F.A. ROIG. 1986. La vegetación del paso de Uspallata Mendoza Argentina. Veröff. Geobot. Inst. ETH Stiftung Rübel 91: 141-150.

ANAND, M. \& I. ORLÓCI. 1997. Chaotic dynamics in a multispecies community. Environ. Ecolog. Stat. 4: 337-344.

ANCHORENA, J. 1985. Recursos naturales y aptitud de uso ganadero. Dos cartas en escala 1:40.000 para la región magallánica. In: Boelcke, Moore et Roig (eds.), Transecta Botánica de la Patagonia Austral: 695-734. CONICET, Instituto de la Patagonia y Royal Society, Buenos Aires.
ANDERSON, D.L., J.A. DEL AGUILA \& A.E. BERNARDON. 1970. Las formaciones vegetales en la Provincia de San Luis. Revista Invest. Agropec. INTA, Ser. 2, Biol. 7 (3): 153-183.

AUER, V. 1933a. Verschiebungen der Wald und Steppengebiete Feuerlands in postglazialer Zeit. In: Results of the Finnish Exped. to Tierra del Fuego in 1928-1929. Acta Geogr. Soc. Geogr. Fenn. 5 (2). Helsinki.

AUER, V. 1933b. Die Moore Südamerikas insbesondere Feuerlands. Handb. d. Moorkunde hrsg. K. v. BÜLOW 7: 224-242.

AUER, V. 1939. Der Kampf zwischen Wald und Steppe auf Feuerland. Peterm. Mitt.: 193-197.

AUER, V. 1941. Der Torf und die Torfschichten als historische Urkunden Feuerlands und Patagoniens. Geol. Rdschau. 32: 647-671.

AUER, V. 1963. Die geographischen Gebiete der Moore Feuerlands. Mitt. Friink. Geogr. Ges. 10: 31-38.

AZARA. F. DE. 1809. Voyage dans l'Amerique méridionale. 4 voi. Paris. (Trad. Berlin 1810, Madrid 1923).

BÁEZ, J.R. 1922. Breve noticia sobre la vegetación de los Dep. Paraná y Diamante (Entre Ríos). Bol. Minist. Agric. 27: 465-475.

BÁEZ, J.R. 1938. Observaciones fitogeográficos sobre la Pampa occidental de la Provincia de Córdoba. Revista Argent. Agron. 5: 165-170.

BÁEZ, J.R. 1939. Dos aspectos de la vegetación del Norte de San Luis. Physis 15: 357-376.

BÁEZ, J.R. 1942. Regiones forestales de Entre Ríos. Minist. Agric. Nac. Comis. Prov. Pro Exposic. Forest., Paraná.

BÁEZ, J.R. 1956. La vegetación del Parque Provincial General San Martín. Mem. Mus. Entre Ríos 33, Bot. Paraná.

BÁEZ, J.R. 1969. Mapa fitogeográfico de la Provincia de Entre Ríos. GAEA 5: 65. 1937. Reimpresión en: A. Burkart (Ed.): Flora Ilustrada de Entre Ríos (Argentina). Colecc. Científ. INTA VI, 2. Buenos Aires.

BAILEY WILLIS. 1914. El norte de la Patagonia, naturaleza y riquezas. Minist. Obr. Publ., Buenos Aires.

BARDAT, J., F. BIORET, M. BOTINEAU, V. BOULLET, R. DELPECH, J. GEHU, J. HAURY, A. LACOSTE, J. RAMEAU, J. ROYER, G. ROUX \& J. TOUFFECT. 2004. Prodrome des végétations de France. Patrimoines Naturals 61: Publications scientifiques du Muséum national d'histoire naturelle. Paris.

BARKMAN, J.J., J. MORAVEC \& S. RAUSCHERT. 1986. Code of phytosociological nomenclatures. Vegetatio 67: 145-196.

BATISTA, W., A. ROLHAUSER, F. BIGANZOLI, S. BURKART, L. GOVETO, A. MARANTA, 


\section{E. Martínez Carretero et al. - Prodromus Sinsistemático y Estudios Fitosociológicos}

A.G. PIGNATARO, N. MORANDEIRA \& M. RABADÁN. 2014. Las comunidades vegetales de la sabana del Parque Nacional El Palmar (Argentina). Darwiniana n.s. 2: 5-38.

BERTILLER, M. 1993. Estepas subarbustivo-herbáceas de Nassauvia glomerulossa y Poa dusenii del centrosur del Chubut. In: Paruelo J.M., M.B. Bertiller, T. Schlichter \& F. Coronato (eds.). Secuencias de deterioro en distintos ambientes patagónicos. Su caracterización mediante el modelo de estados $y$ transiciones. Convenio Argentino-Alemán de Cooperación Técnica INTA-GTZ. Proyecto LUDEPA-SME, San Carlos de Bariloche.

BERTOLAMI, M. 1990. La vegetación periurbana de Mendoza. Transecta Cricyt-Gato de Monte. Parodiana 6: 211-225.

BIONDI, E., E. FEOLI \& V. ZUCCARELLO. 2004. Modelling environmental responses of plant associations: A review of Some critical concepts in vegetation study. Crit. Rev. Plant Sci. 23: 149-156.

BIONDI, E. 2011. Phytosociology today: Methodological and conceptual evolution. Plant Biosyst. 145 (Supl.): 19-29.

BIRGER, S. 1906. Die Vegetation bei Port Stanley auf den Falklandsinseln. Bot. Jahrb. 39: 275-305.

BÖCHER, T.W. 1958. Plantgeografiske undersøgelser iAndes i det vestlige Argentina. Státens Almindel. Videnskabsfond Arsberetning: 7-12.

BÖCHER, T.W., J. HJERTING \& K. RAHN. 1972. Botanical studies in the Atuel valley area Mendoza province Argentina III. Dansk Bot. Arkiv 22: 191358.

BOELCKE, O. 1957. Comunidades herbáceas del Norte de Patagonia y sus relaciones con la ganadería. Revista Invest. Agric. 11: 5-98.

BONVISUTTO, E. 1989. Estudio de la vegetación en una comunidad ubicada entre Puerto Blest y Puerto Alegre (Laguna Frías) en el Parque Nacional Nahuel Huapi. Tesis de Licenciatura, Centro Regional Universitario Bariloche, Universidad Nacional del Comahue, Bariloche.

BORGNIA, M., A. MAGGI, M. ARRIAGA, B. VILÁ, B. AUED \& M.H. CASSINI. 2006. Caracterización de la vegetación en la Reserva de Biósfera Laguna Blanca (Catamarca, Argentina). Ecología Austral 16: 29-45.

BORHIDI, A. 1996. Phytogeography and vegetation ecology of Cuba. Akademici Kiadó.

BORHIDI, A., O. MUÑÍZ \& E. DEL RISCO. 1979. Clasificación fitocenológica de la vegetación de Cuba. Acta Bot. Acad. Sci. Hung. 25: 263-301.

BORHIDI, A., O. MUÑIZ \& E. DEL RISCO. 1983. Plants communities of Cuba. I. Fresh and salt water, swamp and coastal vegetation. Acta Bot. Hund. 29: 337-376.
BORISOV, J., C. CAROSIO \& L. CIUFFO. 1991. Las comunidades vegetales de la cuenca del lago Potrero de Los Funes (San Luis, Argentina). Parodiana 6: 303-322.

BRACKEBUSCH, L. 1893. Über die Bodenverhiiltnisse des nordwestlichefl Teiles der Argentinischen Republik mit Bezugnahme auf die Vegetation. Peterm. Mitt. 39: 153-166.

BRAGADIN, E.A. 1959. Las pasturas en la región de Los Llanos (Provincia de La Rioja). Estudio sinecológico de las asociaciones desde el punto de vista forrajero. Revista Agron. Noroeste Argent. 3: 289-334.

BRAN, D., V. NAKAMATSU \& J. BARRIA. 1985. La vegetación del área comprendida entre los lagos San Martín y Viedma Prov. de Santa Cruz. INTA, inéd.

BRAUN-BLANQUET, J. 1915. Les Cé'vennes méridionales. Arch. des Sc. phys. et Nat. $2^{\circ}$ sér. 39 et 40 .

BRAUN-BLANQUET, J. 1921. Prinzipien einer Systematik der Pflanzengesellschaften auf floristischer Grundlage, Jahrb. St. Gallischen Naturwiss. Ges. 57: 346.

BRAUN-BLANQUET, J. 1928. Pflanzensoziologie. Grundzüge der Vegetationskunde. Springer, Berlin.

BRAUN-BLANQUET, J. 1931. Aperçu des groupements végétaux du Bas-Languedoc. Commun. Stat. Int. Geobot. Medit. Alp. (SIGMA), Montpellier 9: 35-40.

BRAUN-BLANQUET, J. 1948. La végétation alpine des Pyrénées orientales. (Commun. Stat. Int. Géobot. Médit. Alpine 98) Mon. Est. Pir. \& Inst. Esp. Edafol. Ecol. Fisiol. Veg. 9: 7-306.

BRAUN-BLANQUET, J. 1958. Lagunenverlandung und Vegetationsetnwicklung an der franzözischen Mittelmeerküste bei Palavas, ein Sukzessionsexperiment. Comm. S.I.G.M.A., 141: 9-32.

BRAUN-BLANQUET, J. 1964. Pflanzensoziologie. Grundzüge der Vegetationskunde. Ed. 3. Springer, Wien.

BRAUN-BLANQUET, J. \& J. PAVILLARD. 1928. Vocabulaire de Sociologie végétale. $3 \mathrm{Ed}$. Montepellier.

BRAUN-BLANQUET, J., W. GAJEWSKI, M. WRABER \& J. WALAS. 1936. Classe des RuderetoSecalinetales. Groupements messicoles, culturaux et nitrophiles-rudérales du cercle de végétation méditerranéen. Prodr. Group. Vég. 3, Montpellier.

BRAUN-BLANQUET, J., L. EMBERGER \& R. MOLINIER. 1947. Instructions pour l'établissement de la Carte des groupements végétaux. Causse Graille Castelnau, Montpellier.

BRAUN-BLANQUET, J. \& R. TÜXEN. 1952. Irishe Pflanzengesellschaften. Veröff. Ver. Geobot. Inst. E.T.H. Stiffung Rübel 25: 22-421. 
BRAUN-BLANQUET, J. \& ORIOL DE BOLOS. 1958. Les groupements végétaux du bassin moyen de l'Ebre et leur dynamisme. Anal. Est. Exp. Aula Dei, 5: 1-266.

BRULLO, S. \& C. MARCENO. 1980. Il Diplotaxion erucoidis in Sicilia, con considerazioni sulla sintassonomia e distribuzione. Notiz. Fitosoc. 15: $27-44$.

BURKART, A. 1957. Ojeada sinóptica sobre la vegetación del delta del Río Paraná. Darwiniana 11: 457-561.

BURKART, A. 1969. Característica de la vegetación extratropical en América del Sur. Darwiniana 15: 556-557.

BURKART, S., R. LEÓN \& C. MOVIA. 1990. Inventario fitosociológico del pastizal de la Depresión del Salado (Prov. Bs.As.) en un área representativa de sus principales ambientes. Darwiniana 30: 27-69.

BURMEISTER, H. 1861. Reise durch die La Plata-Staaten mit besonderer Rücksicht auf die Argentinische Republik 1857-1860. 2 vol. Halle.

CABIDO, M. \& A.ACOSTA. 1985. Estudio fitosociológico en bosques de Polylepis australis en las Sierras de Córdoba, Argentina. Doc. phytosociol., nouv. sér. 9: 385-400.

CABIDO, M., A. ACOSTA SOLÍS \& S. DÍAZ 1989. Estudios fitosociológicos en los pastizales de la sierras de Córdoba Argentinas. Las comunidades de la Pampa de San Luis. Phytocoenología 17: 569-592.

CABIDO, M., A. ACOSTA \& S. DÍAZ. 1990. The vascular flora and the vegetation of granitic outcrops in the upper Córdoba mountains (Argentina). Phytocoenologia 19: 267-281.

CABIDO, M., M. CARRANZA, A. ACOSTA \& S. PÁEZ. 1991. Contribución al conocimiento fitosociológico del bosque chaqueño-serrano en la prov. de Córdoba Argentina. Phytocoenologia 19: 547-566.

CABIDO, M., A. MANZUR, L. CARRANZA \& C. GONZÁLEZ ALBARRACIN. 1994. La vegetación y el medio físico del Chaco Árido en la provincia de Córdoba, Argentina Central. Phytocoenologia 24: 423-460.

CABRERA, A.L. 1934. Vegetación. En: R. Maldonado Bruzzone. Informe de la Comisión Exploradora 4. Comis. Centr. Invest. sobre la Langosta: 13-17.

CABRERA, A.L. 1936. Apuntes sobre la vegetación de las dunas de Juancho. Notas Mus. LaPlata 1, Bot. 8.

CABRERA, A.L. 1940. La vegetación espontánea de las dunas de Miramar. Dir. Agr. Ganad. e Ind. Ministerio de Obras Públicas Buenos Aires 20: 5-17.

CABRERA, A.L. 1941. Las comunidades vegetales de las dunas costaneras de la Prov. de Buenos Aires. DAGI La Plata.

CABRERA, A.L. 1943a. Vegetación. En: J.M. Torres, A.L. Cabrera y D. Daireaux. Estudio fisiográfico del Partido de Las Conchas. Reseñ. Inst. Agrar. Argent. 3 (1-4): 33-40.
CABRERA, A.L. 1943b. Vegetación del Partido de San Isidro. Reseñ. Inst. Agrar. Argent. 3 (18): 137-140.

CABRERA, A.L. 1943c. Vegetación del Partido de Junín. Reseñ. Inst. Agrar. Argent. 4 (23): 97-100.

CABRERA, A.L. 1944. Vegetación del Partido de Chascomús. Reseñ. Inst. Agrar. Argent. 4 (29): 50-55.

CABRERA, A.L. 1945. Apuntes sobre la vegetación del partido de Pellegrini. DAGI III: 1-93.

CABRERA, A.L. 1948a. Las comunidades vegetales de los alrededores de La Plata. Lilloa 20: 269-376.

CABRERA, A.L. 1948b. Notas sobre la vegetación de la Puna Argentina. Anales de la Acad. Nac. C.E.F.yN. 12: $15-38$.

CABRERA, A.L. 1949. Las comunidades vegetales de los alrededores de La Plata. Ministerio de Agricultura y Ganadería. Inst. de Bot. Publ. Técnica 51, Buenos Aires.

CABRERA, A.L. 1955. Vegetation of the arid and semiarid regions of LatinAmerica. Plant Ecology Rev. UNESCO, Res. sur la zone aride 6: 77-113.

CABRERA, A.L. 1958. La vegetación de la Puna argentina. Revista lnvest. Agric. 11: 317-412.

CABRERA, A.L. 1968. Ecología Vegetal de la Puna. Proc. UNESCO México Sympos. 1966, Geo-Ecología de las Regiones Montañosas de las Américas Tropicales. Colloq. Geogr. 9: 91-116.

CABRERA, A.L. 1969. Vegetación de la Provincia de Buenos Aires. En: A.L. Cabrera (Ed.): Flora de la Provincia de Buenos Aires. Colecc. Científ. INTA IV (1):101-134.

CABRERA, A.L. 1971. Fitogeografía de la República Argentina. Bol. Soc. Argent. Bot. 14: 1-42.

CABRERA, A.L. 1976. Regiones Fitogeográficas Argentinas. ACME Ed., Buenos Aires.

CABRERA, A.L. 1977. Notas sobre algunas comunidades altoandinas del NE de la Patagonia. Obra del Centenario del Mus. La Plata III: 213-227.

CABRERA, A.L. \& D. ABBIATTI. 1945. Excursión botánica a las provincias de Salta y Jujuy. Revista Mus. La Plata, Secc. ofic. 1944: 142-153.

CABRERA, A.L. \& G. DAWSON. 1944. La selva marginal de Punta Lara en la ribera argentina del río de La Plata. Revista Mus. La Plata V: 267-299.

CABRERA, A.L., R. KIESLING \& E.M. ZARDINI. 1977. Notas sobre algunas comunidades altoandinas del noroeste de Patagonia. Obra del Centenario del Museo de La Plata 3: 213-227.

CABRERA, A.L. \& A. WILLINK. 1973. Biogeografia de América Latína. Ser. de Biol. Monogr. 13. Progr. Reg. Desarrollo Científ. y Tecnol. Secret. Gener. OEA, Washington.

CANTERO, J.J., A. CANTERO \& J.M. CISNEROS. 1996. La vegetación de los paisajes hidrohalomorficos del centro de Argentina. Universidad Nacional de Río Cuarto. 


\section{E. Martínez Carretero et al. - Prodromus Sinsistemático y Estudios Fitosociológicos}

CANO, E., 1990. Rasgos principales de los pastizales de la provincia de La Pampa. Revista Fac. Agr. UN La Pampa 5: 1-14.

CANO, E. \& C. MOVIA. 1967. Utilidad de la fotointerpretación de las comunidades vegetales del bosque de caldén. Inst. de Bot. Agr. Serie Fitogeogr. $\mathrm{n}^{\circ} 4$.

CANO, E. \& R. GOMEZ CADRET, 1968. Índice bibliográfico de mapas de vegetación de la Argentina. La Vegetac. de la Rep. Argentina. Ser. Fitogeogr. INTA 9.

CANO, E., B. FERNÁNDEZ \& M. MONTES. 1980. Vegetación. En: INTA. Inventario de los recursos naturales de la prov. de La Pampa.

CANO, E., H.D. ESTERLICH \& B. FERNÁNDEZ. 1988a. Comunidades vegetales en una catena topográfica de área medanosa Departamento Conhelo-La Pampa-Argentina. Revista Fac. Agr. 3: 31-43.

CANO, E., H.D. ESTERLICH, B. FERNÁNDEZ \& E. MORICI. 1988b. Tres pastizales ubicados en una catena topográfica en el sudeste de La PampaArgentina. Revista Fac. Agr. 3: 85-95.

CARETTE, E. \& A. RUIZ LEAL, 1939. La flora mendocina, bosquejo fitogeográfico. En: R. Morey. Album de Mendoza: 1-13.

CARNEVALI, R. 1994. Fitogeografía de la provincia de Corrientes. Corrientes. Gob. Provincia de Corrientes-INTA.

CARRANZA, M.L., M. CABIDO, A. ACOSTA \& S. PAEZ. 1993. Las comunidades vegetales del Parque Natural Provincial y Reserva Forestal Natural Chancani, Provincia de Córdoba. Lilloa 38: 75-92.

CASTEllanos, A. 1927. Por un rincón de la Puna de Atacama. Asoc. Cult. Confer. Rosario 6.

CASTELLANOS, A. 1938. Facies de "El Monte" en la Sierra de la Ventana. Lilloa 2: 5-11.

CASTELLANOS, A. 1958. Observaciones sobre la vegetación del Occidente de Formosa. Bol. Acad. Ci. Córdoba 40: 229- 263.

CERAZZO, B. \& L. CONTICELLO. 2007. La vegetación en bordes de rutas del Alto Valle de Río Negro Argentina. Multequina 16: 99-121.

CERAZZO, B. \& L. CONTICELLO. 2008. Comunidades de malezas en cultivos en la provincia de Neuquén (Argentina). Bol. Soc. Argent. Bot. 43: 121-135.

CHICHIZOLA, S.E. 1993. Las comunidades vegetales de la Reserva Natural Estricta Otamendi y sus relaciones con el ambiente. Parodiana 8: 227-263.

CINGOLANI, A.M., D. BRAN, C. LÓPEZ \& J. AYESA. 2000. Comunidades vegetales y ambiente en el ecotono boreal entre los distritos patagónicos Central y Occidental (Río Negro, Argentina). Ecología Austral 10: 47-61.
CLAISSE, R. 1977. La Méthode phytosociologique appliquée à l'etude des paisages urbains. Essai de transposition. Thése Doct. Etat. Pharm. Lille.

CLAISSE, R. \& J. M. GEHU. 1978. Application de la methode phytosociologique á l'analysse des paysages urbains et ruraux. Symp. Assoziationskomplexe (Sigmeten) 363-374, J. Kramer, Vaduz.

CLEMENTS, F.E. 1904. The development and structure of vegetation. Studies in the vegetation of the State. III. Bot. Surv. Nebraska 7: 1-175.

CLEMENTS, F.E. 1916. Plant succession. An analysis of the development of vegetation. Cornell University Library. Carnegie Institution of Washington, Publication No. 242.

CLEMENTS, F.E. 1928. Plant succession and indicators. H.W. Wilson, Nueva York.

COLLANTES, M. \& J.P. LEWIS. 1980. Ordenamiento de las comunidades vegetales herbáceas del Departamento Rosario (Prov. Sta. Fe Argentina). ECOSUR 7: 171-184.

COLLANTES, M. \& J.P. LEWIS. 1981. La vegetación de la prov. de Santa Fe IV. Análisis de las comunidades herbáceas del Dpto. Rosario. Bol. Soc. Argent. Bot. 19: 115-138.

COLLANTES, M., J. ONTIVEROS \& O. BIANCIOTTO. 1990. Análisis de las comunidades de Nothofagus de Tierra del Fuego. Parodiana 6: 185-195.

CONTICELlO, L., A. BUSTAMANTE \& M.B. CERAZO. 2008. Sintaxones ruderales y adventicios en la zona del Alto Valle de Río Negro y Neuquén. Multequina 17: 55-71.

CONTICELLO, L., R. GANDULLO, A. BUSTAMANTE \& C. TARTAGLIA. 1996. Fitosociología de los bosques caducifolios del norte del Dpto. Lácar y sur de Huiliches, Neuquén. Bosque 17: 27-43.

CONTICELLO, L., R. GANDULLO, A. BUSTAMANTE \& C. TARTAGLIA. 1997. El Distichlo-Salicornietum ass. nov. en banquinas del Alto Valle de Río Negro y Neuquén. Actas XIII Congreso Latinoamericano de Malezas.

CONTICELLO, L., R. GANDULLO, A. BUSTAMANTE \& C. TARTAGLIA. 1998. Estudio fitosociológico de malezas ruderales en rutas nacionales del Alto Valle del Río Negro y Neuquén. I. Parodiana 11: 75-98.

CORREA LUNA, H. 1955. Reserva Nacional Finca El Rey, su valor como representante de una importante región fitogeográfica. Natura (An. Parqu. Nac.) 1: 113-120.

CORREA LUNA, H. 1964. Parque Nacional Tierra del Fuego. Aspecto físico y fitogeográfico. An. Parqu. Nac. 10: 61-72.

CZAJKA, W. \& F. VERVOORST, 1956. Die naturraumliche Gliederung Nordwest-Argentiniens. Peterm. Mitt.: 89-102, 196-208. Gotha. 
D’ANGELO, C., D.E. PRADO, S.L. STOFELLA \& J.P. LEWIS. 1987. The subchaquenian vegetation of Santa Fe (Argentina). Phytocoenologia 15: 329-352.

DALMASSO, A. 1997. El meloncillo Malesherbia lirana var. scabrifolia en laderas altoandinas de suelos sueltos. Multequina 6: 99-101.

DALMASSO, A., E. MARTÍNEZ CARRETERO, F. VIDELA, S PUIG \& R. CANDIA. 1999. Reserva Natural de Villavicencio, Mendoza, Argentina. Plan de manejo. Multequina 8: 11-50.

DALMASSO, A. \& J. MÁRQUEZ. 2004. Vegetación de la Pampa del Acequión y alrededores (San Juan). Multequina 13: 15-31.

DE MARCO, A., F.A. ROIG \& C. WUILLOUD. 1993. Vegetación del piedemonte andino en el centro oeste de Mendoza. Multequina 2: 201-241.

DEVITO, H.A. 1951. Potrero de los Funes. Rasgos fitogeográficos de la zona. Act. XV Sem. Geogr. Mendoza.

DÍAZ GONZÁLEZ, T.E. 2004. Pasado, presente y futuro de la Fitosociología española. Lazaroa 25: 3-13.

DIERSCHKE, H. 1994. Pflanzensoziologie. Grundlagen und Methoden. Verlag. Eugen Ulmer, Stuttgart.

DIMITRI, M.J. 1959. La protección de la flora en el Noreste de San Luis. An. Parqu. Nac. 8: 63-86.

DIMITRI, M.J. 1960. Parques Nacionales. La Argentina, Suma de Geografía 5: 449-498.

DIMITRI, M.J. 1962. La flora andino-patagonica. An. Parqu. Nac. 9: 1-115.

DIMITRI, M.J. 1972a. Consideraciones generales. En: M.J. Dimitri (Ed.): La región de los bosques andinopatagónicos, sinopsis general. Colecc. Cient. INTA 10: 15-26. Buenos Aires.

DIMITRI, M.J. 1972b. Consideraciones sobre la determinación de la superficie y los límites naturales de la región andino-patagónica. Colecc. Cient. INTA 10: 59-79.

DIMITRI, M.J. 1972c. Ecología de la región andinopatagónica. Colecc. Cientif. INTA 10: 111-124.

DIMITRI, M.J., A.R. Volkart de Hualde, A. de Brizuela \& F.A. Tiburcio Fano. 1974. La flora arbórea del Parque Nacional Iguazú. An. Parqu. Nac. 12: 1-180.

DOLLENZ, O. 1985-86. Relevamientos fitosociológicos en la península Muñoz Gamero Magallanes. An. Inst. de la Patagonia 16: 55-62.

DONAT, A. 1931. Über Pflanzenverbreitung und Vereisung in Patagonien. Ber. dtsch. bot. Ges. 49: 403-413.

DONAT, A. 1932. Zur regionalen Gliederung der Vegetation Patagoniens. Ber. dtsch. bot. Ges. 50: 429-436.

DONAT, A. 1934. Zur Begrenzung des Magellanischen Florengebietes. Ber. dtsch. bot. Ges. 52: 131-142.

DONAT. A. 1935. Problemas fitogeográficos relativos a la región magallanica. Revista Argent. Agron. 2: 86-95.
DONAT, A. 1936. Bericht über die pflanzengeographischen Ergebnisse der Inlandeis Expedition der argentinischen Geographischen Gesellschaften. Gaea11, 1933. Ber. dtsch. bot. Ges. 54: 27-46.

DONAT, A. 1937. Die regionale Gliederung der Vegetation Patagoniens. Lasso 4 (7): 8-15.

DREHWALD, U. 1990. Pflanzensoziologische Untersuchungen an Fliessgewasser-Gesellschaften in NO Argentinien. Tuexenia 10: 147-151.

DREHWALD, U. 1995. Epiphytische Pfelnzengesellschaften in NO-Argentinien. Diss. Bot., Band 250.

DREHWALD, U. \& M.E. REINER-DREHWALD. 1996. Orthotricho-Frullanietum bonariensis, una nueva asociación epífita de Buenos Aires, Argentina. Cryptogamie Bryol. Lichénol. 17: 279- 286.

DUDLEY, T.R. \& G.E. CROW. 1983. A contribution to the flora and vegetation of Isla de los Estados (Staten Island), Tierra del Fuego, Argentina. Amer. Geophysical Union Antarctic Res. Series 37.

DUSÉN, P. 1898. Über die Vegetationder feuerlandischen Inselgruppe. Bot. Jahrb. 24: 179-196.

DUSÉN, P. 1905. Die Pflanzenvereine der Magellansländer nebst einem Beitrage zur Okologie der magellanischen Vegetation. Wiss. Ergebn. Schwed. Exped. n.d. Magellanes Hindern, 1895-1897. Stockholm.

DUVIGNEAUD, P. 1946. La variabilité des Associations végétales. Bull. Soc. Roy. Bot. Belg. 78: 107-134.

DU RIETZ, E. 1921. Zur methodologischen Grundlage der modernen Pflanzensoziologie.

ELLENBERG, H. 1954. Zur Entwicklung der Vegetationssystematik in Mitteleuropa. Angew. Pfl. Soziol.,(Wien), Festschr. Aichinger 1:133-143.

ELLENBERG, H. 1956. Aufgaben und Methoden der Vegetationskunde. In : Walter, H. (Hrsg.), Einführung in die Phytologie IV/1. Eugen Ulmer, Stuttgart.

ELLENBERG, H. 1988. Vegetation ecology of central Europe. Cambridge University Press.

ELLENBERG, H. \& D. MUELLER-DOMBOIS. 1967. Tentative physiognomic-ecological classification of plant formations of the earth. Ber. Geob. Inst. E. Tech. Hochshule S. Rübel. Heft 37: 21-55. Zurich.

ENTROCASSI FASSINATO, G. 2015. Estudio de los bosques subtropicales de montaña de la Reserva Ecológica de Uso Múltiple Serranías de Zapla (Jujuy, Argentina): Composición florística, distribución de la vegetación y caracterización bioclimática. Tesis Doctoral. Universidad Complutense de Madrid, Fac. Farmacia, Dept. Biología Vegetal II.

ESKUCHE, U. 1968. Fisonomía y Sociología de los bosques de Nothofagus dombeyi en la región de Nahuel Huapi. Vegetatio 16:192-204.

ESKUCHE, U. 1969. Berberitzengebüsche und Nothofagus antarctica-wälder in Nordwestpatagonien. Vegetatio 19: 264-285. 


\section{E. Martínez Carretero et al. - Prodromus Sinsistemático y Estudios Fitosociológicos}

ESKUCHE, U. 1973a. Pflanzengesellschaten der Küstendünen von Argentinien, Uruguay und Südbrasilien. Vegetatio 28: 201-250.

ESKUCHE, U. 1973b. Estudios fitosociológicos en el norte de la Patagonia. 1. Investigación de algunos factores de ambiente en comunidades de bosque y de chaparral. Phytocoenologia 1: 64-113.

ESKUCHE, U. 1975. Análisis corológico y sistemático del Lindernio-Mercadonietum hernarioidis. Phytocoenologia 2: 261-269.

ESKUCHE, U. 1979. La vegetación de las dunas al sur de la confluencia de los ríos Paraná y Paraguay. Doc. phytosociol., nouv. sér. IV: 267-265.

ESKUCHE, U. 1984. Vegetationsgebiete von Nord und Mittelargentinien. Phytocoenologia 12: 185-199.

ESKUCHE, U. 1986. Bericht über Internationale Pflanzengeographische Exkursion durch Nordargentinien (1983). Veröff. Geobot. Inst. Stiftung Rübel 91: 12-117.

ESKUCHE, U. 1992a. Sinopsis cenosistemática preliminar de los pajonales mesófilos seminaturales del noreste de la Argentina incluyendo pastizales pampeanos y puntanos. Phytocoenología 21: 237312.

ESKUCHE, U. 1992b. La vegetación de las dunas marítimas de América Latina. Bosque 13: 23-28.

ESKUCHE, U. 1992c. Los espartillares un monumento histórico precolombiano en el tapiz vegetal de nordeste argentino. Guía por flora y vegetación alrededor de la confluencia de los ríos Paraná y Paraguay 1: 1-37. Publicación del Herbarium Humboldtianum Facultad de Ciencias Exactas, Naturales y Agrimensura, Universidad Nac. del Nordeste, Corrientes.

ESKUCHE, U. 1998. Estudios fitosociológicos en el NW de la Patagonia. III. El Arrayanal y la Pradera arbolada en sustitución del bosque de Coíhue y Ciprés. Folia Bot. Geobot. Correntesiana 13: 1-17.

ESKUCHE, U. 1999. Estudios fitosociológicos en el norte de la Patagonia. II. Los bosques de Nothofagus dombeyi. Phytocoenología 29: 177-252.

ESKUCHE, U. 2002.Pflanzensociologische Untersuchungen in Nordpatagonien. IV. Die Wälder des Nothofagion pumilionis. Folia Bot. Geobot. Correntesiana 16:1-47.

ESKUCHE, U. 2004. La vegetación de la vega del río Paraná Medio superior, Argentina. Folia Bot. Geobot. Correntesiana 17: 1-60.

ESKUCHE, U. 2005. Las comunidades vegetales litorales de los lagos patagónicos y de los canales magallánicos. Folia Bot. Geobot. Correntesiana 18: $1-31$.

ESKUCHE, U. \& ROMERO FONSECA. 1982. Contribución a la biología floral de Wolfiela lingulata (Lemnaceae). Bol. Soc. Argent. Bot. 21: 259-266.
ESKUCHE, U. \& J. L. FONTANA. 1996a. La vegetación de las islas argentinas del Alto Paraná I. Las comunidades de bosque. Folia Bot. et Geobot. Correntesiana 11: 1-13.

ESKUCHE, U. \& J. L. FONTANA. 1996b. La vegetación de las islas argentinas del Alto Paraná. II Las comunidades vegetales del campo abierto. Folia Bot. Geobot. Correntesiana 12: 3-15.

ESKUCHE, U. \& D. IRIART. 1996a. Las comunidades vegetales peridomésticas de Barranqueras Prov. del Chaco Argentina. Folia Bot. Geobot. Correntesiana 10: 3-15.

ESKUCHE, U. \& D. IRIART. 1996b. Tipificaciones en los Ambrosietea elatioris. Folia Bot. et Geobot. Correntesiana 12:16.

EWALD, J. 2003. A critique for phytosociology. J. Veg. Sci. 14: 291-296.

FAGGI, A.M. 1983. Características estructurales y nutricionales de comunidades vegetales del sur de Santa Cruz. Parodiana 2: 277-296.

FAGGI, A.M. 1985a. Cartas de vegetación real y potencial de la Ea. Cabo Buen Tiempo. Parodiana 3: 341-364.

FAGGI, A.M. 1985b. Las comunidades vegetales de Río Gallegos Santa Cruz. En: Boelcke, Moore et Roig (eds.), Transecta Botánica de la Patagonia Austral: 634-694. CONICET, Instituto de la Patagonia y Royal Society, Buenos Aires.

FAGGI, A.M. 1986. Mapa de vegetación de Alsina, provincia de Buenos Aires. Parodiana 4: 381-400.

FAGGI, A.M. 1995. Carta de vegetación de una estancia nordpatagónica. GAEA. Contribuciones Científicas: 59-63

FAGGI, A.M. \& M. ENCABO. 1987. Notas sobre la vegetación azonal del norte de la prov. de Buenos Aires I. Barrancas de Lima. Parodiana 5: 89-99.

FAGGI, A.M. \& A.M. CAGNONI. 1991. La vegetación de Punta Rasa prov. de Buenos Aires. Parodiana 6: 363-374.

FEOLI, E. 1998. Exploring multidimensional space in vegetation science. In: Computer methods in Investigation of the Structure and Functionning of the Vegetation Cover: 143-156. Kazmierczak. Nienartowicz, E., A. Piernik, A. \& J. WilkonMichalska (Eds.), Wydaw Univ. Mikolaja Kopernica, Torun.

FIEBRIG, C. \& T. ROJAS. 1933. Ensayo fitogeográfico sobre el Chaco Boreal. Revista Jard. Bot. Mus. Hist. Nat. Paraguay 3: 3-87.

FINCKH, M. 1996. Die Wälder des VillarricaNationalparks (Südchile). Lebensgemeinschaften als Grundlage für ein Schutzkonzept. Diss. Bot. 259: 181.

FLAHAULT, CH. 1897. PI. IX - Essai d une carte botanique et forestière de la France. Feuille de Perpignan. Annales de Gèographie 6 (28): 384. 
FLORES-TORO, L. \& R. HILDEBRAND-VOGEL. 2006. Estudio fitosociológico de los bosques de Nothofagus pumilio (P. et E.) Krasser del Centro-Sur de Chile. Anales Mus. Hist. Nat. Valparaíso.

FONTANA, J.L. 1991a. Los pajonales mesófilos seminaturales de Misiones (Argentina) I. El Ocimo neurophylli-Elionuretum mutici. Estudios ecológicos $y$ propuestas de conservación. Mém. Unité d'Ecologie et de Biogéographie Univ. Catholique de Louvaina La Neuve.

FONTANA, J.L. 1991b. Las comunidades vegetales de una laguna chaqueña del valle del río Paraná. Folia Bot. Geobot. Correntesiana 6: 1-17.

FONTANA, J.L. 1996. Los pajonales mesófilos seminaturales de Misiones (Argentina). Phytocoenologia 26: 179-271.

FONTANA, J.L. 1998. Análisis sistemático-ecológico de la flora del sur de Misiones (Argentina). Candollea 53: 211-300.

FONTANA, J.L. 2014. La Vegetación reófila del Nordeste argentino. Las comunidades vegetales con Podostemaceae de la Provincia de Misiones. Bol. Soc. Argent. Bot. 49: 115-136.

FONTANA, J.L. 2015. Flora y Vegetación del Nordeste de Corrientes y sur de Misiones. En: V. Bauni, M.Homberg \& V. Capmourteres (Edts.). El Patrimonio Natural y Cultural en el área de influencia del Embalse de Yacyretá, Argentina. Cap. 1: 9-27. Fundac. Felix de Azara, Buenos Aires.

FRANCESCHI, E.A. 1996. The ruderal vegetation of Rosario City, Argentina. Landsc. Urban Plan. 34: 11-18.

FRANCESCHI, E.A., D.E. PRADO \& J.P. LEWIS. 1985. Comunidades vegetales y mapa de la vegetación de la reserva El Rico e islas aledañas prov. de Santa $\mathrm{Fe}$, Argenina. Fac. de Cs. Agr., Univ. Nac. Rosario.

FRANCESCHI, E.A. \& D.E. PRADO. 1989. Distribution of herbaceous communities of the River Paraná valley along an elevation gradient after a catastrophic flood. Conoses 4: 47-53.

FRANGI, J. 1976. Descripción florístico-estructural de un „stand“ de bosque de Nothofagus dombeyi en Lago Gutierrez (Prov. de Río Negro). Darwiniana 20: 577-585.

FRANGI, J. 1985. Sinopsis de las comunidades vegetales y el medio de las Sierras de Tandil (Provincia de Buenos Aires). Bol. Soc. Argent. Bot. 16: 293-319.

FREIBERG, H.M. 1985. Vegetationskuntliche Untersuchungen an Südchilenischen Vulkanen. Bonn.Geograph Abhandlungen Heft 70.

FRIES, R.E. 1905. Zur Kenntnis der alpinen Flora im nórdlichen Argentinien. Nova Acta Reg. Soc. Se. Upsaliensis ser. 4, 1 (4): 1-205.

FUNES, G. \& M. CABIDO. 2008. Relaciones florísticas y características regenerativas en Apurimacia dolichocarpa (Fabaceae), especie endémica del centro de Argentina. Phytocoenologia 38: 1-9.

GALÁN DE MERA, A. \& G. NAVARRO. 1992. Comunidades vegetales acuáticas del Paraguay occidental. Caldasia 17: 35-46.

GALÁN DE MERA, A., M.W. ROSA \& C. CÁCERES. 2002. Una aproximación sintaxonómica sobre la vegetación del Perú, Clases, órdenes y alianzas. Acta Bot. Malacitana 27: 75-103.

GALÁN DE MERA, A. \& V. ORELLANA. 1996. Las comunidades con Corryocactus brevistylus del sur del Perú. Phytologia 80: 40-47.

GALÁN DE MERA, A. \& V. ORELLANA. 2006. Aproximación al esquema sintaxonómico de la vegetación de la región del Caribe y América del Sur. Anales de Biología 28: 3-27.

GALERA, M. 1980. Estudio ecológico de la estepa serrana en las Sierras Chicas de Córdoba. I. Descripción fisonómica estructura y composición. Factores determinantes de grupos ecológicos. Ecología 5: 49-98.

GALLOPIN, G. 1978. Estudio ecológico integrado de la cuenca del Río Manso Superior (Río Negro, Argentina). I. Descripción general de la cuenca. $A n$. Parqu. Nac. 14: 161-230.

GANDULLO, R. 2003. Fitosociología de los matorrales de "ñire" (Nothofagus antarctica) bosque de pehuén (Araucaria araucana) del parque prov. Copahue. I Interpretación sintaxonómica. Candollea 58:164181.

GANDULLO, R. 2004. Nueva asociación de ambientes salinos. Multequina 13: 33-37.

GANDULLO R. \& A.M. FAGGI. 2003. Análisis fitosociológico de los mallines del piso andino del Parque Prov. Copahue Neuquén Argentina. Kurtziana 30: 45-55.

GANDULLO R. \& A.M. FAGGI. 2005. Interpretación sintaxonómica de los humedales del noroeste de la provincia de Neuquén, Argentina. Darwiniana 43: 10-29.

GANDULLO, R. \& A.M. FAGGI. 2006. La vegetación rupícola del Parque Provincial Copahue. Neuquén, Argentina. Kurtziana 32: 13-24.

GANDULLO, R., J. GASTIAZORO \& A. BÜNLI. 1998. Dinamismo de la vegetación psamófila de Senillosa y Plottier Neuquén. Argentina. Multequina 7: 11:20.

GANDULLO, R. \& J. GASTIAZORO. 2009. Suaedetum neuquenensis nueva asociación de ambientes salinos. Multequina 18: 31-36.

GANDULLO, R., J. GASTIAZORO \& P. SCHMID 2010. Vegetación de los barreales del norte central de la provincia del Neuquén. Revista Mus. Argent. Ci. Nat., n.s. 12: 1-4.

GAUCH, H.G. \& R.H. WHITTAKER. 1972. Coenocline simulation. Ecology 53: 446-451. 


\section{E. Martínez Carretero et al. - Prodromus Sinsistemático y Estudios Fitosociológicos}

GEHU, J.M. 1975. Essai systématique et corologique sur les principales associations végétales du littoral atlantique francais. Anales R. Acad. Fanll. Madrid 41: 207-227.

GEHU, J.M. \& S. RIVAS-MARTINEZ. 1981. Notions fondamentales de phytosociologie. In: Dierschke, $\mathrm{H}$. (ed.) Syntaxonomie. Ber. Intern. Symposium IV-V: 5-53. Ed. Cramer, Vaduz.

GÉHU, J.M. 2006. Dictionnaire de sociologie et synécologie végétales. J. Cramer, Berlin-Stuttgart.

GLEASON, H.A. 1917. The structure and development of plant association. J. Torrey Bot. Soc. 44: 463-481.

GLEASON, H.A. 1939. The individualistic concept of the plant association. Amer. Midland Nat. 21: 92110 .

GÓMEZ, D., E. HAENE, S. KRAPOVICKAS, M. BARBARSKAS, J, SANGUINETI, R. BURKART, J. CHÁVEZ \& G. GIL. 1997. Ecoregiones de la Argentina. Reseña y líneas de acción para su conservación. Documento de Avance, Buenos Aires.

GONZÁLEZ LOYARTE, M. 1989a. Los pastizales de piedemonte: los coironales preandinos de Tupungato, Mendoza, Argentina. En: Roig, F. (Ed.), Detección y Control de la Desertificación: 242-253.

GONZÁLEZ LOYARTE, M. 1989b. La acción de los roedores en las modificaciones del relieve y su relación con la vegetación. En: Roig, F. (Ed.), Detección y Control de la Desertificación: 294-307.

GONZÁlEZ LOYARTE, M., M. BUCK \& O.S. TRIONE. 2000. Análisis comparativo de dos comunidades en el bosque de Prosopis flexuosa del NE de Mendoza, Argentina. Multequina 9: 75-89.

GOODALL, D.W. 1952. Quantitative aspects of plant distribution. Biol. Rev. 27: 194-245.

GOODALL, R.N.P. 1972. Tierra del Fuego. $2^{\mathrm{a}}$ ed. Buenos Aires.

GRONDONA, M.F. 1951. Observaciones fitogeográficos en la zona de San Augustin del Valle Fértil. Act. XV Sem. Geogr. Mendoza.

GUTTE, P. 1980. Beitrag zur Kenntnis zentralperuanischer Pflanzegesellschaten II. Die Hoch andinen Moore und ihre Kontaktgesellschaften. Fed. Rep. 91: 327336

GUTTE, P. 1985. Beitrag zur Kenntnis zentralperuanischer Pflanzengesellschaften IV.Die Grassreiche Vegetation der alpinen Stufe. Wiss. Z. Karl Marx Univ. Naturwiss. 34: 537-401.

GUTTE, P. 1986 Beitrag zur Kenntnis zentralperuanischer Pflanzengesellschaften III. Pflanzellschaften der sudalpinen Stufe. Fed. Rep. 97: 319-371.

GUTTE, P. 1988. Der anthropogene Einflu $\beta$ in der PunaRegion Zentralperus. Flora 180 : 31-36.

HAUMAN, L. 1913a. Etude phytogéographique de la région du Río Negro inferieur. Anal. Mus. Nac. Ci. Nat. 24: 279-444.
HAUMAN, L. 1913b. La forêt valdivienne et ses limites. Rec. Inst. Bot. Léo Errera 9: 346-408. Bruxelles; et: Trab. Inst. Bot. y Farm. 34, Buenos Aires (1916).

HAUMAN, L. 1918. La végétation des hautes cordilleres de Mendoza. Anal. Soc. Ci. Argent. 86: 121-188, 225-348.

HAUMAN, L. 1919. La vegetación primitiva de la ribera argentina del Río de la Plata. Revista Centro Estud. Agron. y Vet. 96: 345-355.

HAUMAN, L. 1920a. Un Viaje botánico al Lago Argentino. Anal. Soc. Ci. Argent. 89: 179-281.

HAUMAN, L. 1920b. Ganadería y Geobotánica en la Argentina. Revista Centro Estud. Agron. Vet. 102.

HAUMAN, L. 1925. La végétation de l'ile de Martin García dans le Río de la Plata. Publ. Inst. Invest. Geogr. 10: 1-39.

HAUMAN, L. 1926. Étude phytogéographique de la Patagonie. Bull. Soc. Roy. Belgique, Bot. 58: 105180.

HAUMAN, L. 1931. Esquisse phitogégraphique de l'argentine subtropicale et ses relations avec la Géobotanique sudamericaine. Bull. Soc. Roy. Belgique, Bot. 64: 20-80.

HEIM, A. 1941. Waldzerstorung in Neuseeland und Patagonien. Schweizer Zeitschr. f. Forstwes. 1. Bern.

HEMSLEY, W.B. 1886. Vegetation of South Georgia. Nature 34: 106-107.

HERZOG, Th. 1931. Baum (Wald) und Schneegrenzen in den Kordilleren von Südamerika. Mitt. Geogr. Ges. Jena 39: 72-89.

HIERONYMUS, D.G. 1874- Observaciones sobre la vegetación de la provincia de Tucumán. Bol. Acad. Ci. Córdoba 1: 183-234, 299-423.

HILDEBRAND-VOGEL, R. 1983. Die Vegetation der Tieflandsgebüsche des Sudchilenischen. Lorbeerwaldgebiets unter besonderer Berücksichtigung der Neophytenproblematic. Phytocoenologia 11: 145-223.

HILDEBRAND-VOGEL, R. 2002. Structure and dynamics of southern Chilean natural forests with special reference to the relation of evergreen versus deciduous elements. Folia Geobot. 37: 107-128.

HILDEBRAND-VOGEL, R., R. Goday \& A. Vogel. 1990. Subantarctic-Andean Nothofagus pumilio forest. Vegetatio 89: 55-68.

HILGERT, N.I. \& C. D'ANGELO. 1996. Las comunidades vegetales de los Dptos. Castellanos y Las Colonias (Santa Fe, Argentina). Bol. Soc. Argent. Bot. 32: 3-16.

HILGERT, N.I., J.F. PENSIERO, G. MARINO, J.P. LEWIS \& C. D'ANGELO. 2003. Vegetation of the Saladillo area (province of Santa Fe) in the south of the Chaco, Argentina. Interciencia 28 (9): 1-10.

HOFF, M. \& H. BRISSE. 1983. Proposition d'un schema synthetique des vegetations secundaires intertropicales. Colloq. Phytosociol. 12: 249-267. 
HOLMBERG, E.L. 1895. La flora de la República Argentina. Segundo Censo de la Rep. Argentina: 385-474.

HOSSEUS, C.C. 1915. Expedición al valle y a las fuentes del Río Nirihueo y al Cerro Colorado en el Valle de Pichileufli. Bol. Minist. Agric. 19: 471-515.

HOSSEUS, C.C. 1916a. En las montañas riojanas al oeste del Nevado de Famatina y en regiones limítrofes de la provincia de San Juan. Anal. Soc. Ci. Argent. 82: 11-59.

HOSSEUS, C.C. 1916b. El proyectado Parque Nacional del Sud. Bol. Minist. Agric. Nac.: 3-56.

HOSSEUS, C.C. 1922. Estudios comparativos sobre la vegetación de las provincias de La Rioja y de San Juan. Bol. Acad. Ci. Córdoba 26: 5-160.

HUECK, K. 1950. Estudio ecológico y fitosociológico de los médanos de Cafayate (Salta). Lilloa 23: $63-$ 116.

HUECK, K. 1951a. Vegetationskarten aus Argentinien. Die Erde 1950/51 (2): 145-154.

HUECK, K. 1951b. Dos problemas fitogeográficos de la cuenca de Andalgalá. Bol. Soc. Argent. Bot. 3 : 224-234.

HUECK, K. 1952. Die Araucarien walder des nordlichen Patagonien. Z. Weltforstwirtschaft 15 (4). BerlinGrünau.

HUECK, K. 1953. Urlandschaft, Raublandschaft und Kulturlandschaft in der Provinz Tucumán im nordwestlichen Argentinien. Bonner geogr. Abh. 10. Bonn.

HUECK, K. 1954. Der Anden-Erlenwald (die Alnus jorullensis-Assoziation) in der Provinz Tucumán (Argentinien). Ausgewandte Pflanzensoziologie. Veröffentlichungen des Kärntner Ländesinstituts für angewandte in Klagenfurt. Festschrift Aichinger. I Band.

HUECK, K. 1955. Plantas e fomaçäo organogenica das dunas no litoral Paulista. Parte I. Contribuçöes para a pesquisa fitosociologica paulista 2. Sao Paulo.

HUECK, K. 1957. Las regiones forestales de Sur América. Bol. Inst. Forest. Latino Amer. 2. Merida.

HUECK, K. 1959. Bosques secos de la zona tropical y subtropical de la América del Sur. Bol. Inst. Forest. Latino Amer. 4. Merida.

HUECK, K. 1960/61. Die waldgeographischen Regionen und Unterregionen von Südamerika. Geogr. Taschenbuch: 224-234. Wiesbaden.

HUECK, K. 1966. Die Willder Südamerikas. Okologie, Zusammensetzung und wirtschaftliche Bedeutung. Jena.

HUNZIKER, J.H. 1952. Las comunidades vegetales de la cordillera de La Rioja. Revista Invest. Agrar. 6: 167-196.

IBAÑEZ, F.M. 1962. Vegetación de la Provincia de Entre Ríos. Nordeste 4: 93-127.
IHERING, H. VON. 1929. Klima und Flora von Westpatagonien im Wandel der Zeit. Peterm. Mitt.: 175-180, 240-245, 308-311. Gotha.

IRIART, D. 2016. Comunidades vegetales urbanas del norte de Argentina: variabilidad floristica $y$ corológica. Tesis Doctoral, Universidad Nacional de Córdoba.

IZCO, J. 1998. Types of rarity of plant communities. $J$. Veg. Sci. 9: 641-646.

KALELA, E.K. 1941. Über die Holzarten und die durch die klimatischen Verhaltnisse verursachten Holzartenwechsel. in den waldern Ostpatagoniens. Ann. Acad. Sc. Fenn. Ser. A, 4 Biol., 2.

KALELA, E.A. 1946. Über die Steppen und Wüstenvegetation in Patagonien. Sitzber. Finrl. Akad. Wiss. 1945: 161-172. Helsinki,

KANDUS, P., A.I. MALVÁREZ \& N. MADANES. 2003. Estudio de la comunidades de plantas herbáceas de las islas bonaerenses del bajo del delta del Río Paraná (Argentina). Darwiniana 41: 1-16.

KANTER, H. 1936. Der Gran Chaco und seine Randgebiete. Abh. Geb. Auslandskunde Hans. Univ., Hamburg.

KLIKA, J. \& NOVÁK, V. 1941. Praktikum rostlinné M. ChytrÝ et al:: Nomenclature adjustments of the Arctic, alpine and oro-Mediterranean vegetation sociologie, puidoznalství, klimatologie a ekologie. Melantrich, Praha.

KNAPP, R. 1964. Höhere Vegetationseinheiten einige Gebiete der holarktische unf neotropischen Floren Reiche. Geobot. Mitt. 28: 1-11.

KNAPP, R. 1965. Hohere Vegetationseinheiten von SüdPatagonien und Feuerland. Geobot. Mitt. 35: 1-4.

KOCH, W. 1926. Die Vegetationseinheiten der Linthebene. Jb. St. Gall. Naturwiss. Ges. 61: 1-146.

KOUTCHÉ. S. 1928. Los bosques de Misiones en la región de Yerbal Viejo. Direcc. Grl. Tierras, Minist. Agric. Buenos Aires.

KÜHN, F. 1930. Geografía de la Argentina. Barcelona y Buenos Aires.

KÜHN, F. \& G. Rohmeder. 1943. Estudio fisiográfico de las sierras de Tucumán. Inst. Estud. Geogr. Univ. Nac. Tucumán.

KÜHNEMANN, O. 1970. Vegetación marina de la ría de Puerto Deseado. Opera Lilloana 17.

KÜHNEMANN, O. 1972. Bosquejo fitogeográfico de la vegetación marina del Litoral argentino. Physis 31 (82): 117-142.

KÜHNEMANN, O. 1976. Observaciones ecológicas sobre la vegetación marina y terrestre de la Isla de los Estados (Tierra del Fuego, Argentina). Ecosur 3: 121-248.

KURTZ, F. 1886. Informe preliminar de un viaje botánico, efectuado por órden de la Academía Nacional de Ciencias en Córdoba, en las provincias de Córdoba, 


\section{E. Martínez Carretero et al. - Prodromus Sinsistemático y Estudios Fitosociológicos}

San Luis y Mendoza hasta la frontera de Chile, en los meses de diciembre 1885 a febrero de 1886. Bol. Acad. Ci. Córdoba 9: 349-370.

LANDOLT, E. 1986. The family of Lemnaceae-a monographic study I. Veröff. Geobot. Inst. ETH Stiftg. Rübel Zurich.

LANDOLT, E. 1999. Pleustonic communities with Lemnaceae in South America. Appl. Veg. Sci. 2: $7-16$.

LÁNÍKOVÁ, D. 2009. XC Artemisietea vulgaris Lohmeyer et al. ex von Rochow 1951. In: Chytrý M. (Ed.),Vegetace České republiky. 2. Ruderální, plevelová, skalní a sutová vegetace [Vegetation of the Czech Republic 2. Ruderal, weed, rock and scree vegetation]. Academia Praha: 207-209.

LEÓN, R. J. C. \& A. SUERO. 1962. Las comunidades de malezas de los maizales y su valor indicador. Revista Argent. Agron. 29: 23-28.

LEÓN, R. \& M. AGUIAR. 1985. El deterioro por uso pastoril en estepas patagónicas. Phytocoenologia 13: 181-196.

LEÓN, R.C., D. BRAN, M. COLLANTES, J.M. PARUELO \& A. SORIANO. 1998. Grandes unidades de vegetación de la Patagonia extraandina. Ecología Austral 8: 125-144.

LÉONARD J. 1950. Botanique du Congo Belge. I. Les groupements végétaux du Congo Belge. Encyclopédie du Congo Belge: 45-389, Ed. Believeld, Bruxelles.

LEWIS, P. \& M.B. COLLANTES. 1974. La vegetación de la Provincia de Santa Fé. I. Reseña general y enfoque del problema. Bol. Soc. Argent. Bot. 15: 343-356.

LEWIS, P. \& M.B. COLLANTES. 1975. La vegetación de la Provincia de Santa Fé. II. Las comunidades vegetales del Departamento Rosario. Bol. Soc. Argent. Bot. 16: 151-179.

LEWIS, P., M.B. COLLANTES \& E. PIRE. 1976. La vegetación de la Provincia de Santa Fe III. Las comunidades vegetales del Departamento de San Lorenzo. Bol. Soc. Argent. Bot. 17: 219-237.

LEWIS, J.P., E.F. PIRE, N.J. CARNEVALE, S.I BOCCANELLI, S.L STOFELLA \& D.E. PRADO. 1984. Los pastizales de Stipa y comunidades afines del suroeste de Santa Fe (Argentina). Studia Oecologica 5: 55-76.

LEWIS, J.P., N.J. CARNEVALE, E.F. PIRE, S.I. BOCCANELLI, S.L. STOFELLA \& D.E. PRADO. 1985a. Las comunidades halófilas del sureste de Santa Fe (Argentina). Stud. Oecol. 6: 57-73.

LEWIS, J.P., S.I. BOCCANELLI, N.J. CARNEVALLE, E.F. PIRE, S.L. STOFELLA \& D.E. PRADO. 1985b. Las comunidades higrófilas del sureste de Santa Fe (Argentina). Stud. Oecol. 6: 75-90.
LEWIS, J.P., S.L. STOFELLA, D.E. PRADO, N.J. CARNEVALE, E.F. PIRE \& S.I. BOCCANELLI. 1985c. Pajonales y comunidades misceláneas del sureste de Santa Fe (Argentina). Stud. Oecol. 6: 91103.

LEWIS, J.P., E.A. FRANCESCHI \& D.E. PRADO. 1987. Effect of extraordinary floods on the dynamics of tall grasslands of the river Paraná. Phytocoenologia 15:235-251.

LEWIS, J.P., E.F. PIRE, D.E. PRADO, S.L. STOFELLA, E.A. FRANCESCHI \& N.J. CARNEVALE. 1990. Plant communities and phytogeographical position of a large depression of the Great Chaco. Vegetatio 86: $25-38$.

LEWIS, J.P., E.F. PIRE \& J.L. VESPRINI. 1994. The mixed dense forest of the Southern Chaco. Contribution to the study of flora and vegetation of the Chaco VIII. Candollea 49: 159-168.

LEWIS, J.P. \& E.F. PIRE. 1996. Los Mogotes de Palo Azul (Cyclolepis genistoides Don) de áreas deprimidas de la Región Chaqueña. Revista Asoc. Ci. Nat. Litoral 27: 109-117.

LEWIS, J.P., E.F. PIRE \& I.M. BARBERIS. 1997. Structure, physiognomy and floristic composition of a Schinopsis balansae (Anacardiaceae) forest of the Southern Chaco (Argentina). Revista Biol. Trop. 45: 1013-1020.

LEWIS, J.P., E. PIRE, I. BARBERIS \& D. PRADO. 2006. Los bosques del Espinal periestépico en las proximidades de la localidad de Coronda, Provincia de Santa Fe (Argentina). Revista Inv. Fac. C. Agrar. 10: 1-11.

LEWIS, J.P., S. NOETINGER, D.E. PRADO \& I.M. BARBERIS. 2009. Woody vegetation structure and composition of the last relicts of Espinal vegetation in subtropical Argentina. Biodiv. Conserv. 18: 36153628.

LILLO, M. 1919. Reseña fitogeográfica de la provincia de Tucumán. $1^{\mathrm{a}}$ Reun. Nac.

LJUNGNER, E. 1939. A forest section through the Andes of Northern Patagonia. Svensk. Bot. Tidskr. 33: 321337. Uppsala. Soc. Argent. Ci. Nat. Secc. III Bot.: 210-232.

LORENTZ, P.G. 1876. Cuadro de la vegetación de la República Argentina. En: NAPP. La República Argentina. Buenos Aires.

LORENTZ, P.G. 1878. La vegetación del nordeste de la provincia de Entre Ríos. Buenos Aires. ( $2^{\circ}$ ed., Paraná 1947).

LUEBERT, F. \& R. GAJARDO. 2000. Vegetación de los Andes áridos del norte de Chile. Lazaroa 21: 111-130.

LUEBERT, F. \& R. GAJARDO, 2005. Vegetación altoandina de Parinacota (norte de Chile) y una sinópsis de la vegetación de la Puna meridional. Phytocoenologia 35: 79-128. 
LUEBERT, F., R. GAJARDO \& M. ESTAY. 2003. Nota fitosociológica sobre las asociaciones forestales del Parque Nacional Tolhuaca (Chile). Bol. Mus. Nac. Hist. Nat. Chile 52: 51-66.

LOIDI, J. 1994. Phytosociology applied to nature conservation and land management in Spain. In: Song Y., Dierschke H. \& Wang X. (red.). Appl. Veg. Ecol.: 17-30. Proceedings of the 35 th symposium of I.A.V.S. East China Normal University Press. Shangai.

LOIDI, J. 1999. Preserving biodiversity in the European Union: the Habitats Directive and its application in Spain. Plant Biosyst. 133: 99-106.

LOIDI, J. 2001. Reflexiones sobre la Fitosociología en el momento actual. Quercetea 3: 5-20.

LOIDI, J. 2004. La Fitosociología como elemento renovador de la Botánica española en la segunda mitad del siglo XX. Lazaroa 25: 15-21.

LUTI, R., M. DE SOLIS, M. GALERA, N. DE FERREIRA, M. BERZAL, M. NORES, M. HERRERA \& J. BARRERA. 1979. Vegetación. En: Vázquez, J., R. Miatello \& M. Roque (Eds.), Geografía Física de la Provincia de Córdoba: $297-$ 368.

MACKENZIE LAMB, L. 1958. La vegetación liquénica de los Parques Nacionales patagónicos. An. Parqu. Nac. 7.

MANZI, R. 1961. Aspectos de la vegetación de la Provincia de Santa Fe. Rasgos fitogeográficos del Departamento de San Jerónimo. $1^{\mathrm{a}}$ Reun. Trab. y Com. Cienc. Nat. y Geogr. del Litoral Argent.: 7386. Santa Fe.

MARINO, G. \& J. PENSIERO. 2003. Heterogeneidad florística y estructural de los bosques de Schinopsis balansae (Anacardiaceae) en el sur del Chaco Húmedo. Darwiniana 41: 17-28.

MARTIN, R.R. 1949. Consideraciones generales sobre la flora actual de las turberas de Tierra del Fuego. Revista Argent. Agron. 16: 149-155.

MARTIN, R.R. 1955. Los turbales y bosques fueginos. Acta Geogr. Soc. Geogr. Fenn. 14: 286-290. Helsinki.

MARTÍNEZ, O.G. \& D.E. PRADO. 2013. Distribución fitogeográfica de Helechos y Licófitas en el Valle de Lerma (Salta - Argentina). Chloris chilensis 16 (2).

MARTÍNEZ CARRETERO, E. 1984. El incendio en la vegetación de la Precordillera mendocina III. Los pastizales disclimáxicos en la quebrada de Villavicencio. Parodiana 3: 175-184.

MARTÍNEZ CARRETERO, E. 1985. La vegetación de la Reserva Natural Divisadero Largo (Mendoza Argentina). Doc. phytosociol., nouv. sér. 9: 25-49.

MARTÍNEZ CARRETERO, E. 1987. Observaciones ecológicas sobre Guindilia dissecta (Sapindaceae) en los Andes de Uspallata. Bol. Soc. Argent. Bot. 25: $1-10$.
MARTÍNEZ CARRETERO, E. 1993. La vegetación del sector cerro Casa de Piedra-San Isidro. Multequina 2: $89-140$.

MARTÍNEZ CARRETERO, E. 1993. Sintaxones arvenses ruderales adventicios presentes en la vegetación argentina. Multequina 2: 195-200.

MARTÍNEZ CARRETERO, E. 1994. La vegetación de los Andes centrales de la Argentina. En: Excursión botánica: 28-33.

MARTÍNEZ CARRETERO, E. 1995. La Puna Argentina: delimitación general y división en distritos florísticos. Bol. Soc. Argent. Bot. 31: 27-40.

MARTÍNEZ CARRETERO, E. 1997. The Puna vegetation in the valley of Cazadero Grande Catamarca Province Argentina. Candollea 52: 497-508.

MARTÍNEZ CARRETERO, E. 1999. Saxicolous and riparian vegetation of a piedmont in central-western Argentina. JAE 42: 305-317.

MARTÍNEZ CARRETERO, E. 2000. Vegetación de los Andes centrales de la Argentina. El valle de Uspallata. Bol. Soc. Argent. Bot. 34: 127-148.

MARTÍNEZ CARRETERO, E. 2001. Esquema sintaxonómico de la vegetación de las regiones salinas de la Argentina. Multequina 10: 67-74.

MARTÍNEZ CARRETERO, E. 2004. La Provincia fitogeográfica de la Payunia. Análisis florísticocorológico. Tesis doctoral, F.C.E.F.N., Univ. Nac. Córdoba.

MARTÍNEZ CARRETERO, E. 2005. La Provincia Fitogeográfica de la Payunia. Bol. Soc. Argent. Bot. 39: 195-226.

MARTÍNEZ CARRETERO, E. 2013. La Diagonal Árida Argentina. Entidad bioclimática. En: Pérez, D, A. Rovere y M. E. Araujo (Eds.), Restauración Ecológica en la Diagonal Árida de la Argentina.

MARTÍNEZ CARRETERO, E. 2015. Geoffroea subtropicalis (Lillo) Martínez Carretero comb. \& stat. nov. (Fabaceae-Dalbergiae), and its phytogeographical significance. Phyton 55: 1-16.

MARTÍNEZ CARRETERO, E. \& E. MÉNDEZ. 1992. La vegetación de la vertiente oriental de la cordillera Real Mendoza Argentina. Multequina 1: 1-99-106.

MARTÍNEZ CARRETERO, E. \& F.A. ROIG. 1992. El paisaje en los estudios de la vegetación. Un ensayo para la Patgaonia mendocina. Parodiana 7:165-178.

MARTÍNEZ CARRETERO, E. \& F.A. ROIG. 1994. Asarina antirrhiniflora (Scrophulariaceae) enredadera anfitrópica de la Reserva del Divisadero Largo Mendoza, Argentina. Multequina 3: 3-7.

MARTÍNEZ CARRETERO, E. \& A. DALMASSO. 1995. Observaciones sobre la vegetación en áreas de piletas con aguas coproducidas, Barrancas, Mendoza. En: Martínez Carretero, E. y A. Dalmasso (eds.), Mendoza Ambiental. IADIZA (CONICET)-Gob. de Mendoza. 


\section{E. Martínez Carretero et al. - Prodromus Sinsistemático y Estudios Fitosociológicos}

MARTÍNEZ CARRETERO, E., A. DALMASSO, J. MÁRQUEZ \& M. MARTINELLI. 2009. Vegetation of the High Central Andes of Argentina. Plant communities and phytogeographical units in the northwest of San Juan. Candollea 65: 63-96.

MARTÍNEZ CROVETTO, R. 1950. Las malezas de los céspedes en la Capital Federal y alrededores. Revista Invest. Agric. 4: 1-45.

MARTÍNEZ CROVETTO, R. 1962. Estudios ecológicos en los médanos del sur de Entre Ríos I. Dinámica de la vegetación. Bonplandia 1: 85-141.

MARTÍNEZ CROVETTO, R. 1963. Esquema fitogeográfico de la Provincia de Misiones (República Argentina). Bonplandia 1: 171-223.

MARTÍNEZ CROVETTO, R. 1965. Estudios ecológicos en los campos al sur de Misiones I. Efecto del pastoreo sobre la estructura de la vegetación. Bonplandia 2: 29-73.

MARTÍNEZ CROVETTO, R. 1967. Evolución de la vegetación en una sere secundaria en el Noroeste de Corrientes (República Argentina). Bonplandia 2: 127-135.

MARTÍNEZ CROVETTO, R. 1978. Estudios ecológicos en los campos del sur de Misiones II. La repoblación de los tacurúes. Bonplandia 5: 21-14.

MARTÍNEZ CROVETTO, R. 1980a. El SelaginelloAyenietum o'donellii en el noroeste de la prov. de Corrientes (Argentina). Phytocoenologia 7: 467474.

MARTÍNEZ CROVETTO, R. 1980b. Estudios fitosociológicos en el sotobosque de los quebrachales del NE de la prov. de Corrientes. Bol. Soc. Argent. Bot. 19: 315-329.

MARTÍNEZ CROVETTO, R. \& B. PICCININI. 1950. Los palmares de Butia yatay. Vegetación de la Rep. Argentina. Revista Inv. Agr. 4: 153-242.

MATURO, H.M., L.J. OAKLEY \& D.E. PRADO. 2005. Vegetación y posición fitogeográfica de la Reserva 'El Bagual'. En: Di Giacomo, A.G. y S.F. Krapovickas (Eds.), Historia Natural y Paisaje de la Reserva 'El Bagual', Provincia de Formosa, Argentina. Temas de Naturaleza y Conservación 4: 59-73. Aves Argentinas/Asociación Ornitológica del Plata, Buenos Aires.

MAYOR, M. 1995. Utilidad de las investigaciones de Duvigneaud y Ellenberg en el campo de la ecología de la vegetación. Bol. Ci. Nat. R.I.D.E.A. 43: 93-106.

MÉNDEZ, E. 1971. Relación botánica de un viaje al Payén en el sur mendocino. Deserta 2: 99-105.

MÉNDEZ, E. 1983. Observaciones sobre la flora adventicia de los viñedos en Mendoza. Parodiana 2: $263-276$

MÉNDEZ, E. 1984. Observaciones ecológicas sobre la vegetación adventicia de los cauces de riego en Mendoza. Parodiana 3: 185-196.
MÉNDEZ, E. 1986. Observaciones ecológicas sobre comunidades de malezas y sus relaciones con las prácticas culturales en viñedos de Mendoza. Parodiana 4: 143-152.

MÉNDEZ, E. 1990. Observaciones fitosociológicas de la vegetación adventicia de cultivos hortícolas de la prov. de Mendoza. Parodiana 6: 197-209.

MÉNDEZ, E. 1992. Dinamismo de la vegetación en la Pampa Amarilla. Multequina 1: 73-81.

MÉNDEZ, E. 1994. La vegetación de los Andes centrales. En: Excursión botánica: 34-44.

MÉNDEZ, E. 2005. La vegetación de la Reserva Provincial Laguna de Llancanelo. Candollea 60: 123-148.

MÉNDEZ, E. 2014. La Vegetación de Los Altos Andes Centrales: Bardas Blancas-Paso Pehuenche (MaLargüe, Mendoza, Argentina). Bol. Soc. Argent. Bot. 49: 257-281.

MÉNDEZ, E. 2015. Lamio amplexicaulis-Urticetum urentis Ass. Nov. En viñedos de mendoza, Argentina. Bol. Soc. Argent. Bot. 50: 107-113.

MÉNDEZ, E. \& J.A. AMBROSETTI. 1985. Las comunidades vegetales de Río Turbio-El Turbio Santa Cruz. En: Boelcke, Moore et Roig (Eds.), Transecta Botánica de la Patagonia Austral: 634694.

MÉNDEZ, E., E. MARTÍNEZ CARRETERO \& C. WUILLOUD. 1993. La vegetación de las reservas naturales de la provincia de Mendoza III. La vegetación del campo experimental El Divisadero Sta. Rosa. Parodiana 8: 113-123.

MENDIOLA, M. 2004. Highland grassland vegetation in the Northwestern Andes of Argentina. Vegetation structure and species composition in relation to grazing. Mt. Res. Dev. 24: 243-250.

MEYER, T. 1936. Características de la flora del Departamento de Resistencia. Revista Argent. Agron. 2: 349-358.

MEYER, T. 1940- Informe de una excursión botánica a Tilcara y Departamento Yavi. Mem. Inst. M. Lillo: 26-31.

MEYER, T. 1943. Notas de un viaje botánico al Departamento de Santa Victoria (Salta). Revista Geogr. Americ. 10 (120): 121-132.

MEYER, T. 1944. Un VlaJe botánico al Departamento de Oran (Prov.de Salta). Revista Geogr. Amer. 11: 255-264,

MEYER, T. 1959. Características de la flora salteña, estudios realizados sobre ella y orientación sobre trabajos futuros. Revista Fac. Cienc. Nat. Salta 1: 15-33.

MEYER, T. 1963. Estudios sobre la selva tucumana. La selva de Mirtáceas de Las Pavas. Opera Lilloana 10.

MOGLIA, M. 2006. Fitosociología urbana I: La comunidad Poa annua L. y Coronopus didymus (L.) 
Sm. del centro de Argentina. Acta Bot. Malacitana 31: 131-140.

MONTALDO, P. 1976a. Análisis ecológico de mallines de Aysen. Agro Sur 4: 106-110.

MONTALDO, P. 1976b. Aspectos ecológicos de los coironales de Aysen. Medio Ambiente 2: 12-20.

MONTICELLI, J. 1938. Anotaciones fitogeográficos en la Pampa central. Lilloa 3: 251-382.

MORAVEC, J. 1989. Influences of the individualistic concept of vegetation in Sintaxonomy. Vegetatio 81: 29-39.

MORAVEC, J. 1992. Is the Zürich-Montpellier approach still unknown in vegetation science in Englishspeaking countries. J. Veg. Sci 3: 277-278.

MORELLO, J. 1952. El bosque de Algarrobo y la estepa de Jarilla en el Valle de Santa María (Provincia de Tucumán). Darwiniana 9: 315-347.

MORELLO, J. 1955. Estudios botánicos en las regiones áridas de la Argentina. Ambiente, Morfología y Anatomía de cuatro arbustos resinosos de follaje permanente del Monte. Revista Agron. Noroeste Argent. 1: 301-370.

MORELLO, J. 1958. La provincia fitogeográfica del Monte. Opera Lilloana 2.

MORELLO, J. 1967. Bases para el estudio fitoecológico de los grandes espacios (el Chaco argentino). Cienc. e Invest. 23 (6): 252-267.

MORELlO, J. 1970. Ecología del Chaco. Bol. Soc. Argent. Bot. 11 (supl.): 161-174.

MORELLO, J. \& J. ADAMOLI. 1968. Las grandes unidades de vegetación y ambiente del Chaco argentino. Primera Parte: Objetivos y metodología. La Vegetac. de la Rep. Argentina. Ser. Fitogeogr. INTA 10. Buenos Aires.

MORELLO, J. \& J. ADAMOLI. 1974. Las grandes unidades de vegetación y ambiente del Chaco argentino. Unidades de Vegetación y ambiente del Chaco. La Vegetac. de la Rep. Argentina. Ser. Fitogeogr. INTA 13.

MORELLO, J., N.E. CRUDELI \& M. SARACENO. 1971. Los vinalares de Formosa (República Argentina). La Vegetación de la Rep. Argentina. Ser. Fitogeogr. INTA 11.

MORELLO, J. \& C. SARAVIA TOLEDO. 1959. El bosque chaqueño. I. Paisaje primitivo, paisaje natural y paisaje cultural en el oriente de Salta. II. La ganadería y el bosque en el oriente de Salta. Revista Agron. Noroeste Argent. 3: 5-81, 209-258.

MOTA, J., J.A. GARRIDO, F. MARTÍNEZHERNÁNDEZ, J.M. MEDINA-CAZORLA, E. DANA \& M.L. RODRÍGUEZ-TAMAYO. 2004. Fitosociología y series de vegetación. En: Mota, J., Cabello J, Cerrillo M. \& Rodríguez-Tamayo M. (Eds.), Subdesiertos de Almería naturaleza de cine. Consejería de Medio Ambiente, Junta de Andalucia.
MUCINA, L. \& E. VAN DER MAAREL. 1989. Twenty years of numerical syntaxonomye. Vegetatio 81:115.

MUELLER DOMBOIS, D. \& H. ELLENBERG. 1974. Aims and Methods of Vegetation Ecology. John Wiley \& Sons, Nueva York.

MULLENDERS, W. 1949. Communication préliminaire sur un essai de cartographie pédologique et phytosociologique dans le Haut-Lomami (CongoBelge). Bull. Agr. Congo Belge 40: 511-532.

NAVARRO, G. 1993. Vegetación de Bolivia: el altiplano meridional. Rivasgodaya 7: 69-98.

NAVARRO, G. \& M. Maldonado. 2002. Geografía ecológica de Bolivia. Vegetación y ambientes acuáticos. Fundación Simón I. Patiño, Cochabamba.

NEGER, F.W. 1896. Die Vegetationsverhaltnisse im nordlichen Araukarien. Bot. Jahrb.: 382-411.

NEGER, F.W. 1897. Die Araucarienwalder in Chile und Argentinien. Forstl. naturw Zeitschr.: 416-426. (Trad. Buenos Aires 1944).

NIEDERLEIN, G. 1881. Einige wissenschaftliche Resultate einer argentinischen Expedition nach dem Río Negro (Patagonien). Z. Ges. f. Erdkunde Berlin 16: 48-74, 81-90.

NIEDERLEIN, G. 1883. Einige wissenschaftliche Resultate einer Reise in die südstliche Pampa bis zuro Río Salado. Z. Ges. f. Erdkunde Berlin 18: 305-311.

OBERDORFER, F. 1960. Pflanzensoziologische Studien in Chile. Flora et Vegetatio Mundi 2.

OFFNER, F. 1907. La végétation des iles Falkland. La Géographie 16: 302-303.

ØKLAND, R.H. 1990. Vegetation ecology: theory, methods and applications with reference to Fennoscandia. Sommerfeltia Suppl. 1: 1-233.

ONTIVERO, M. \& E. MARTÍNEZ CARRETERO. 2013. El Ecosistema de Vega en el Corredor Bioceánico (San Juan, Argentina) mediante el empleo de TIG. En: García, A. (Ed.), El Corredor Bioceánico en San Juan. Recursos culturales y naturales del sector andino. Edit. UN San Juan.

ORIOL DE BOLÓS, A.C. 1988. Irradiacions tropicals dins la vegetaciò nitròfila mediterranìa. Acta Bot. Barc. 37: 25-31.

ORIOL DE BOLÓS, A.C., CERVI \& H. HATSCHBACH. 1991. Estudios sobre la vegetación del estado de Paraná (Brasil Meridional). Collectánea Bot. 20: 79-182.

ORIOL DE BOLÓS, A.C. \& F. MASCLANS. 1955. La vegetación de los arrozales en la región mediterránea. Collectánea Bot. 4: 1-32.

ORIOL DE BOLÓS, A.C., CERVI \& G. HATSCHBACH. 1991. Estudios sobre la vegetación del estado de Paraná (Brasil meridional). Collectanea Bot. 20: 79-182. 


\section{E. Martínez Carretero et al. - Prodromus Sinsistemático y Estudios Fitosociológicos}

PARODI, L. 1929. Observaciones sobre la vegetación de las islas cerca al puerto de San Nicolas. Tomo conmem. X: XV Anivers. Fundac. Fac. Agron. y Vet. Buenos Aires: 73-94.

PARODI, L. 1930. Ensayo fitogeográfico sobre el partido de Pergamino. Revista Fac. Agron. y Vet. Buenos Aires 7: 65-271.

PARODI, L. 1934. La vegetación de Reconquista, región de bosques de Espinillos, Palmares y Sabanas de altas Gramineas. Revista Geogr. Amer. 1: 389-400.

PARODI, L. 1940a. La distribución geográfica de los Talares en la Provincia de Buenos Aires. Darwiniana 4: 33-56.

PARODI, L. 1940b. Los bosques naturales de la Provincia de Buenos Aires. An. Acad. Nac. Ci. Exact. Fis. Nat. 7: 79-90.

PARODI, L. 1941. Viaje a la región de Bahía Blanca. Revista Mus. La Plata, secc. ofic. 1940: 69-78.

PARODI, L. 1943. La vegetación del departamento de San Martín en Corrientes (Argentina). Darwiniana 6: 127-177.

PARODI, L. 1961. Ciento cincuenta años de la Botánica en la República Argentina. Bol. Soc. Argent. Bot. 9: 3-68.

PASSARGE, H., 1967. Über Saumgesellschaften im nordostdeutschen Flachland. Fedd. Rep. 74: 145158.

PERALTA, I. 1994. La vegetación de los Andes centrales de la Argentina. En: Excursión botánica: 14-22.

PEREZ-MOREAU, R.A. 1935. Reseña botánica de un viaje a la alta cordillera del NW de San Juan. Revista Centro Estud. Cienc. Nat. 1: 49-59.

PEREZ-MOREAU, R.A. 1944. La Provincia Antartándica. Holmbergia 3: 93-110.

PEREZ-MOREAU, R.A. 1945- Reseña botánica sobre los Parques Nacionals Nahuel Huapi, Los Alerces y Lanín. Anal. Mus. Patagonia 1: 253-278.

PIGNATTI, S. 1990. Towards a Prodrome of plant communities. J. Veg. Sci 1: 425-426.

PIRE, E.F. \& J.P. LEWIS. 1993-1994. Los matorrales de Allenrolfea vaginata (Gris.) O.K. del Chaco santafesino. Revista Asoc. Ci. Nat. Litoral 24 y 25: $1-8$.

POI DE NEIFF, A. \& J.J. NEIFF. 1977. El pleuston de Pistia stratiotes de la Laguna Barranqueras (Chaco, Argentina). Ecosur 4: 69-101.

POLLMANN, W. 2001. Caracterización florística y posición sintaxonómica de los bosques caducifolios de Nothofagus alpina (Poepp. et Endl.) Oerst. en el centro sur de Chile. Phytocoenologia 31: 353-400.

POLLMANN, W. \& R. HILDEBRAND. 2005. Structure and the Composition of Species in Timberline Ecotones of the Southern Andes. In: Mountain Ecosystems, edited by G. Broll and B. Keplin: 117151. Springer, Berlin.
POORE, M.E.D. 1955. The use of phytosociological methods in ecological investigations. I. The Braun-Blanquet system. J. Ecol. 43: 226-244.

POORE, M.E.D. 1962. The method of successive aproximation in descriptive ecology. Adv. Ecol. 43: 606-651.

PRADO, D., E. FRANCESCHI \& M. BIANCHI. 1989. El bosque del escarpe occidental del Río Paraná (Argentina). Composición florística y estructura. Acta Bot. Bras. Supl. 3: 99-108.

PRADO, D.E. 1993a. What is the Gran Chaco vegetation in South America? I: A review. Contribution to the study of flora and vegetation of the Chaco. V. Candollea 48: 145-172.

PRADO, D.E. 1993b. What is the Gran Chaco vegetation in South America? II: A redefinition. Contribution to the study of the flora and vegetation of the Chaco. VII. Candollea 48: 615-629.

PRADO, D.E. 1995. Selva pedemontana: contexto regional y lista florística de un ecosistema en peligro. En: Brown, A.D. \& H.R. Grau (Eds.), Investigación, Conservación y Desarrollo en Selvas Subtropicales de Montaña. L.I.E.Y. (UNT)/Proyecto de Desarrollo Agroforestal, Tucumán.

RAGONESE, A.E. 1936. Algunas consideraciones referentes al límite de los bosques andinopatagonicos. Physis 12: 97-101.

RAGONESE, A.E. 1941. La Vegetación de la provincia de Santa Fe (R.A.). Darwiniana 5: 369-416.

RAGONESE, A.E. 1951. Estudio fitosociológico de las Salinas Grandes. En: Vegetación de la República Argentina. Revista Inv. Agr. 5 (1-2).

RAGONESE, A.E. 1967. Vegetación y ganadería en la República Argentina. Colecc. Científ. INTA 5.

RAGONESe, A.E. \& J. Castiglioni. 1970. La vegetación del Parque Chaqueño. Bol. Soc. Argent. Bot. 11, Supl.: 133-160.

RAGONESE, A.E. \& G. COVAS. 1940. La distribución geográfica de los Quebrachales en la Provincia de Santa Fe. Revista Argent. Agron. 7: 176-184.

RAGONESE, A.E. \& G. COVAS. 1947. La flora halófita del Sur de la Provincia de Santa Fe. Darwiniana 7: 401-496.

RAGONESE, A. \& B. PICCININI. 1969. Límite entre el Monte y el Semidesierto patagónico en las provincias de Río Negro y Neuquén. Bol. Soc. Argent. Bot. 11: 299-302.

RAGONESE, A. \& B. PICCININI. 1977. Consideraciones sobre la vegetación de las Salinas de Mascasin ( $\mathrm{La}$ Rioja-San Juan, República Argentina). Darwiniana 21: $49-60$.

REŠKA, M. s/f. New plants associations of Coast of Tierra del Fuego.

RINGUELET, E. 1938. Estudio fitogeográfico del Rincón de Viedma. Revista Fac. Agron. 21: 15-136. 
RINGUELET, E. 1939. Asociaciones vegetales del Rincón de Viedma (Bahía de Samborombón). Physis 15: 261 289,491-493.

RINGUELET, E. 1947. Geobotánica y Ecología de las altas sierras de Córdoba. Gaea 8: 81-96.

RIVAS-MARTÍNEZ, S. 1975. Sobre la nueva clase Polygono-Poetea annuae. Phytocoenologia 1: 123140

RIVAS-MARTÍNEZ, S. 1985. Biogeografia y Vegetación. Discurso de ingreso como Académico de Número. Real Academia de Ciencias Exactas, Físicas y Naturales, Madrid.

RIVAS-MARTÍNEZ, S. 1987. Nociones sobre Fitosociología, Biogeografía y Bioclimatología. En: Peinado, M. \& S. Rivas-Martínez (eds.), La vegetación de España: 19-45.

RIVAS-MARTÍNEZ, S. 1994. Dynamic-zonal phytosociology as landscape science. Phytocoenologia 24: $23-25$.

RIVAS-MARTÍNEZ, S. 2005. Avances en Geobotánica. Discurso de Apertura del Curso Académico de la Real Academia Nacional de Farmacia del año 2005.

RIVAS-MARTÍNEZ, S. \& O. TOVAR. 1982. Vegetation andinae I. Datos sobre las comunidades vegetales altoandinas de los Andes centrales del Perú. Lazaroa 4: $167-187$

RIVAS-MARTÍNEZ, S., J.C. BASCONES, T.E. DÍAZ, F. FERNÁNDEZ GONZALEZ \& J. LOIDI. 1991. Vegetación del Pirineo occidental y Navarra. Itinera Geobot. 5: 5-456.

RIVAS-MARTÍNEZ, S., T.E. DÍAZ GONZÁLEZ, J.A. FERNÁNDEZ PRIETO, J. LOIDI \& A. PENAS. 1991. Festuco hystricis-Ononidetea striatae y Rosmarinetea officinalis, clases de vegetación independientes. Itinera Geobot. 5: 505-516.

RIVAS-MARTÍNEZ, S., G. NAVARRO, A. PENAS \& M. COSTA. 2011. Biogeographic Map of South America. A preliminary survey. Int. J. Geobot. Res. 1: 21-40.

RODRIGUEZ GARCÍA, D., A. SOUROUILLE, G. GALLOPIN \& C. MONTANA. 1978. Estudio ecológico integrado de la cuenca del Río Manso Superior (Río Negro, Argentina). II. Tipos de vegetación. An. Parqu. Nac. 14: 231-248.

RODWELL, J.S. 1991. Woodlands and scrub. Vol. I. British Plant Communities. Cambridge University Press.

ROHMEDER, W. 1942a. Beobachtungen in den Waldern von Tucumán. Jahrb. dtsch. Volksbund Buenos Aires 1943: 157-166.

ROHMEDER, W. 1942b. Clima y vegetación en las sierras de Tucumán. Asoc. Cult. Confer. Rosario 3.

ROHMEDER, W. 1943. El paisaje entre Jujuy y La Quiaca. Un cuadro sinóptico de la Quebrada de Humahuaca y de la parte adyacente de la Puna jujeña. Gaea 7: 93-97.
ROHMEDER, W. 1945. Paisaje natural y antropogéneo en Tucumán. Gaea 7: 293-315.

ROHMEDER, W. 1947. Investigación fitoestadística en un erial patagonico. Gaea 8: 73-80.

ROHMEDER, W. 1955. Topoclimas y sus relaciones con relieves vegetación y cultivos en el valle de Tafí (Montaña deAconquija, noroeste argentino). Bol. Estud. Geogr. 2: 235-253.

ROIG, F.A. 1955a. La vegetación de la estancia El Yuyalitos, Paso de las Carretas. Mendoza. Bol. Estud. Geogr. 2: 287-289.

ROIG, F.A. 1955b. Contribución al conocimiento de la zona de Ojos del Salado (Catamarca). Bol. Estud. Geogr. 2: 319-336.

ROIG, F.A. 1970. La Reserva Forestal de Ñacuñán. Deserta 1: 21-232.

ROIG, F.A. 1971. Flora y vegetación de la Reserva Forestal de Nacuñán. II. La vegetación. Deserta 1: 201-228.

ROIG, F.A. 1972. Bosquejo fisionómico de la vegetación de la Provincia de Mendoza. Bol. Soc. Argent. Bot. 13, supl.: 49-80.

ROIG, F.A. 1973a. El cuadro fitosociológico en el estudio de la vegetación. Deserta 4: 45-66.

ROIG, F.A. 1973b. Investigaciones climáxicas II. Los pastizales disclimáxicos del Melocotón (Mendoza) y nuevas observaciones sobre la biología de Schinus polygamus. Deserta 4: 173-184.

ROIG, F.A. 1976. Las comunidades vegetales del piedemonte de la precordillera mendocina. Ecosur 3: 1-45.

ROIG, F.A. 1985. Ephedra boelckei (Ephedraceae) nueva especie sudamericana de la sección Alatae Stapff. Parodiana 3: 11-19.

ROIG, F.A. 1989. Ensayo de detección y control de la Desertificación en el $\mathrm{W}$ de la ciudad de Mendoza desde el punto de vista de la vegetación. En: Roig, F. (Ed.), Detección y Control de la Desertificación. 196-232.

ROIG, F.A. 1990. La Fitogeografía y la Fitosociología en la Argentina. Parodiana 6 (1): 129-164.

ROIG, F.A. 1994. Sonchus arvensis. Su comportamiento como maleza. Multequina 3: 45-49.

ROIG, F.A. 1998. La vegetación de la Patagonia. En: Correa M.N. (ed.) Flora patagónica: 48-166. Instituto Nacional de Tecnología Agropecuaria (INTA) Colección Científica 13, Buenos Aires.

ROIG, F.A. 2000. Comunidades vegetales productoras de turba en Tierra del Fuego. En: Disertaciones y conclusiones del Curso-Taller sobre Conservación de Ecosistemas a nivel mundial: 33-454.

ROIG, F.A. \& J.A. AMBROSETTI. 1971. Investigaciones climáxicas I. Restos de un estrato arbóreo bajo de Schinus polygamus en la Precordillera de Mendoza. Deserta 2: 115-130. 


\section{E. Martínez Carretero et al. - Prodromus Sinsistemático y Estudios Fitosociológicos}

ROIG, F., J. ANCHORENA, O. DOLLENZ, A.M. FAGGI \& E. MÉNDEZ. 1985a. Las comunidades vegetales de la Transecta Botánica de la Patagonia Austral I. La vegetación continental. En: Boelcke, Moore et Roig (Eds.), Transecta Botánica de la Patagonia Austral: 350-456. CONICET, Instituto de la Patagonia y Royal Society, Buenos Aires.

ROIG, F., O. DOLLENZ \& E. MÉNDEZ. 1985b. Vegetación de los canales. En: Boelcke, Moore et Roig (Eds.), Transecta Botánica de la Patagonia Austral: 457- 519. CONICET, Instituto de la Patagonia y Royal Society, Buenos Aires.

ROIG, F.A \& E. MARTÍNEZ CARRETERO. 1992. La vegetación actual del valle de Uspallata (Mendoza Argentina) durante los últimos 4500 años a través de restos botánicos arqueológicos. Bamberger Geograph. Schriften 11:283-294.

ROIG, F.A. \& E. MARTÍNEZ CARRETERO. 1998. La vegetación puneña en la provincia de Mendoza. Phytocoenologia 28: 565-608.

ROIG F.A. \& E. PALADINI. 1994. Novedades en Prosopis (algarrobos) para la prov. de Mendoza. Multequina 3: 55-58.

ROIG, F.A. \& V.G. ROIG. 1969. Nuevos datos sobre la corriente florística chaqueña en Mendoza y observaciones sobre Aspidosperma quebrachoblanco en el límite sudoeste de su dispersión. Revista Fac. Ci. Agr. 15: 46-52.

ROIG, F.A. \& F.A. ROIG JUÑENT. 1994. AdesmioAraucarietum, asociación xérica con Araucaria en la estepa patagónica. Resúmenes VI Congreso Latinoamericano de Botánica, Mar del Plata.

ROIG, F.A. \& A. RUIZ LEAL. 1959. El bosque muerto de Guandacol (La Rioja). Revista Agron. Noroeste Argent. 3: 139-145.

ROIG JUÑENT, F.A. 1989. Ejemplo práctico del uso de la Dendrocronología en el estudio del dinamismo de una comunidad arbustiva en el piedemonte de la ciudad de Mendoza. En: Detección y Control de la Desertificación: 185-188.

ROIVAINEN, H. 1954. Studien über die Moore Feuerlands. An. Bot. Soc. Zool. Bot. Fennicae Vanamo, Helsinski.

ROQUERO, M.J. 1968. La vegetación del Parque Nacional Laguna Blanca. An. Parqu. Nac. 11: 129207.

RÜHLE, K. 1928. Die Vegetationsformen Südamerikas in ihrer klimatischen Bedingtheit. Peterm. Mitt.: 2934, 95-100. Gotha.

RUIZ-LEAL, A. 1951. La ruta de la Quebrada de Horcones hasta el Aconcagua. Act. XV Sem. Geogr. Mendoza.

RUIZ-LEAL, A. 1954. Flora de las inmediaciones de Ushuaia, Tierra del Fuego. Revista Fac. Cienc. Agrar. 4.
RUIZ-LEAL, A. 1972. Los confines boreal y austral de las provincias Patagónica y Central respectivamente. Bol. Soc. Argent. Bot. 13, supl.: 89-118.

RUIZ-LEAL, A. \& F. ROIG. 1958. Algunos efectos de la acción de las aguas sobre la vegetación marginal en el Río Olta (La Rioja). Revista Argent. Agron. 25: $102-114$.

RUIZ-LEAL, A. \& F. ROIG. 1959. Erial de vegetación en monticulos. Bol. Estud. Geogr. 6: 161-209.

RUIZ-LEAL, A. \& F. ROIG. 1964. Itinera Gilliesia. Excursiones botánicas Gillesianas 1: Flórula y vegetación del Cerro y Pampa de la Polcura. Bol. Estud. Geogr. 11: 174-192.

RUIZ-LEAL, A. \& F. ROIG. 1965. Itinera Gilliesia. Excursiones botánicas Gillesianas 11: Flórula y vegetación del Alto de los Manantiales. Bol. Estud. Geogr.12: 127-148.

RUTHSATZ, B. 1977. Pflanzengellschaften und ihre Lebensbedingungen in dem Andinen Halbwüsten Nordwest-Argentiniens. Diss. Bot. 39.

RUTHSATZ, B. 1978. Las plantas en cojín de los semidesiertos andinos del Noroeste argentino. Darwiniana 21: 491-539.

RUTHSATZ, B. \& C.P. MOVIA. 1975. Relevamiento de las estepas andinas del Noreste de la Provincia de Jujuy. Fundac. Educ. Cienc. y Cult., Buenos Aires.

SAMEK, V. \& M. ONCADA. 1971. Comunidades vegetales de las lagunas blancas de Pinar del Río, Cuba. Acad. Sci. Cuba, Ser. Pinar del Río 27: 1-29.

SAYAGO, M. 1969. Estudio fitogeográfico del notre de Córdoba. Bol. Acad. Nac. Ci. Córdoba 46: 123-427

SCHENCK, H. 1905. Vergleichende Darstellung der Pflanzengeographie der subantarktischen Inseln 1 insbesonders über Flora und Vegetation von Kerguelen. Mit Einfügung hinterlassener Schriften A.F. W.SCHIMPERS., Wiss. Ergebn. dtsch. Tiefsee Exped. 11 Valdivia 112 (1).

SCHMIEDER, O. 1923. Contribución al conocimiento del Nevado de Chani y de la alta cordillera de Jujuy. Bol. Acad. Nac. Ci. Córdoba 27: 135-166.

SCHMIEDER, O. 1928. Die Entwicklung der Pampa als Kulturlandschaft. Verh. u. wiss. Abh. 22. Dtsch. Geographentag. Karlsruhe 1927. Breslau.

SCHMIEDER, O. 1929. Das Pampaprob1em. Peterm. Mitt. 4: 246-247. Gotha.

SCHMITHÜSEN, J. 1956. Die räumliche Ordnung der chilenischen Vegetation. Bonner Geogr. Abh. 17-89.

SCHREITER, R. 1936. Estudio fisiográfico de la Quebrada del Portugués, Departamento de Monteros (Tucumán). Bol. Mus. Ci.Nat. Tuc.: 33-35.

SCHWABE-BRAUN, A. \& R. TÜXEN. 1981. Lemnetea minoris. Prodromus der europäischen Pflanzengeselschaften, Lief 4. Vaduz. 
SCHWAAR, J. 1981. Amphi-arktische Pflanzengesellschaften in Feuerland. Phytocoenologia 9: 547-572.

SCHULZ, A.G. 1961. Nota sobre la vegetación acuática chaqueña. „Esteros“ y „embalsados“. Bol. Soc. Argent. Bot. 9: 141-150.

SECKT, H. 1912. Contribución al conocimiento de la vegetación del Noroeste de la República Argentina (valles de Calchaqui y Puna de Atacama). Anal. Soc. Ci. Argent. 74: 185-225.

SECKT, H. 1943. Vegetationsverhaltnisse der Argentinischen Republik. Buenos Aires.

SEIBERT, P. 1972. Der Bestandesaufbau einiger Waldgesellschaften in der Südkordillere(Argentinien). Forstwiss. Cbl. 91 (4): 278-291.

SEIBERT, P. 1979. Die Vegetationskarte des Gebietes von El Bolsón prov. Río Negro ud ihre Anwendung in der Landnutzungsplanung. Bonner Geogr. Abhand. Heft 62.

SEIBERT, P. 1982. Carta de vegetación de la región de El Bolsón, Río Negro y su aplicación a la planificación del uso de la tierra. Documenta Phytosociologica 2. Bs. As.

SEIBERT, P. \& X. MENHOFER. 1991-1992. Die Vegetation des Wohngebietes der Kallawaya und des Hochandes von Ulla-Ulla in den Bolivianischer Anden. Phytocoenologia 20: 145-276; 289-438. Berlin-Stuttgart.

SEIBERT, P. 1993. La vegetación de la región de los Kallawaya y del altiplano de Ulla-Ulla en los Andes bolivianos. Ecología en Bolivia 20: 1-84.

SINGER, R. 1971. Forest Mycology and forest communities in South America. II. Mycorrhiza sociology and fungus sucession in the Nothofagus dombeyi-Austrocedrus chilensis woods of Patagonia. In: E. Hacska Ylo (Ed.): Mycorrhizae. Proc. First N. Amer. Confer. on Mycorrhizae, April1969. US Dept. Agric. Forest. Serv. Mise. Publ. 1189: 204-215. Washington, D. C.

SISSINGH, G. 1969. Über die systematische Gliederung von Trittpflanzen Gesellschaften. Mitt. Flor. Soz. Arbeitsgem., N. F., 14: 179-192.

SKOTTSBERG, C. 1904. On the zonal distribution of south athlantic and antarctic vegetation. Geogr. J. 1904: 655662.

SKOTTSBERG, C. 1905. Some remarks upon the geographical distribution of vegetation in the coulder southern hemisphere. Ymer 25: 402-427.

SKOTTSBERG, C. 1906. Observations on the vegetation of the Antarctic Sea. Bot. stud. till. F. R. Kjellman. Uppsala.

SKOTTSBERG, C. 1907. Vegetationsbilder aus Feuerland, von den Falkland Inseln und von Südgeorgien. In: Karsten u. Schenck. Vegetationsbilder 4 (3/4). Jena.

SKOTTSBERG, C. 1909a. Pflanzenphysiognomische Beobachtungen aus dem Feuerlande. Wiss. Ergebn. Schwed. Südpolarexped. 1901-1903, 4(9). Stockholm.
SKOTTSBERG, C. 1909b. Studienüber das Pflanzenleben der Falklandinseln.Wiss. Ergebn. Schwed. Südpolar exped.1901-1903, 4 (10). Stockholm.

SKOTTSBERG, C. 1910. Have we any evidences of postglacial climatic changes in Patagonia and Tierra del Fuego. XI. Internat. Geol. Kongr. Stockholm.

SKOTTSBERG, C. 1913. Botanische Ergebnisse der schwedischen Expedition nach Patagonien und dem Feuerlande 1907-1909. ID. A botanical survey of the Falkland Islands. Stockholm.

SKOTTSBERG, C. 1916. Botanische Ergebnisse der schwedischen Expedition nach Patagonien und dem Feuerlande 1907-1909. V. Die Vegetationsverhälltnisse längs der Cordillera de los Andes S von $420 \mathrm{~S}$ Br. Ein Beitrag zur Kenntnis der Vegetation in Chiloe Westpatagonien dem andinen Patagonien und Feuerland. Kungl. Svenska Vetensk. Hand1. 56 (5). Stockholm.

SKOTTSBERG, C. 1921a. Algunos resultados botánicos obtenidos durante la campaña de la comisión sueca en los territorios australes de Chile y Argentina en los años 1908-1909. Revista Chil. Hist. Nat. 25: 474-494.

SKOTTSBERG, C. 1921b. The vegetation in South Georgia. Wiss.Ergebn. Schwed. Südpolar Exped. 1901-1903, 4 (12). Stockholm.

SKOTTSBERG, C. 1921c. Einige Bemerkungen über die Vegetationsverhältnisse des Graham Landes. Wiss. Ergebn. Schwed. Südpolar Exped. 1901-1903, 4 (13). Trad. Mus. Argent. Cienc. Nat. Buenos Aires.

SKOTTSBERG, C. 1931. Zur Pflanzengeographie Patagoniens. Ber. dtsch. bot. Ges. 49: 481-493.

SKOTTSBERG, C. 1942. The Falkland Islands. Chronica Bot. 7: 23-26. Waltham.

SKOTTSBERG, C. 1945. Tbe Falkland Islands. In: VER DOORN, F. (Ed.), Plants and plant science in Latin America. Waltham.

SKOTTSBERG, C. 1960. Remarks on the Plant Geography of the Southern cold temperate zone. Proc. Roy. Soc. B 152. Stockholm.

SORIANO, A. 1949. El límite entre las provincias botánicas Patagónica y Central en el territorio del Chubut. Lilloa 20: 193-202.

SORIANO, A. 1950. La vegetación del Chubut. Revista Argent. Agron. 17: 30-66.

SORIANO, A. 1956. Los distritos florísticos de la Provincia Patagónica. Revista Invest. Agric.10: 323 347.

STOFELLA, S. 1995. La heterogeneidad florística del pajonal de Spartina argentinensis (Poaceae) en los bajos submeridionales de la provincia de Santa Fe (Argentina). Bol. Soc. Argent. Bot. 31: 95-101.

TÁlAMO, A., J. TOLABA, C. TRUCCO \& E. ACUÑA. 2010. Unidades de vegetación y composición florística en sectores del Altiplano del noroeste de Argentina. I. Ambientes de estepas. Ecología en Bolivia 45: 4-19. 


\section{E. Martínez Carretero et al. - Prodromus Sinsistemático y Estudios Fitosociológicos}

TANQUILEVICH, R. 1971. Los suelos de la Reserva ecológica de Ñacuñán. Deserta 2: 131-206.

TATON, A. 1949. Les principales associations de la région de Nioka et leur valeur agrostologique. Bull. Agric. Congo Belge 40: 1884-1900.

TELL, G. \& C. BONETTO. 1978. Estudios limnológicos en la cuenca del Riachuelo (Corrientes, Argentina). IV. Estudios ecológicos sobre las algas psammíticas de la Laguna Totoras. Ecosur 5: 85-96.

TUR, N.M. 1972. Embalsados y camalotales de la región isleña del Paraná Medio. Darwiniana 17: 397-407.

TWEEDIE, J. 1840. Extracts from a few rough notes of a journey across the pampas of Buenos Ayres to Tucumán, in 1835. An. Nat. Hist. 4. London.

THERBURG, A. 1997. Ökologie der Halophytenvegetation in der Provinz Mendoza, Argentinien (Monte Formation). Diss. Bot. Band 273: 1-181.

TÜXEN. R. 1950. Grundri $\beta$ einer Systematik der nitrophilen Unkrautgesellschaften, in der Eurosibirischen Región Europas -Mitt. Flor. Soziol. Arbeitsgemenschaft N. F. 2: 99, 94. Stolzenau/W.

TÜXEN, R. 1937. Die Pflanzengesellschaften Nordwestdeutschlands. Mitt. Flor. soz. Arbgem. Niedersachsen 3: 1-170.

TÜXENR. 1950. Grundriss einer Systematik der nitrophilen Unkrautgesellschaften in der Eurosibirischen Region Europas. Mitt. Flor. Pflanzesoz. Arbeitsgem., ser. n. 2: $94-175$.

TÜXEN, R. 1956. Die heutige potentielle natürliche Vegetation als Gegenstand der Vegetationskartierung. Angew. Pflanzensoz. 13: 1-55.

TÜXEN, R. 1966. Uber nitrophile Elymus-gesellschaften an nordeuropiiischen, nordjaponischen und nordamerikanischen küsten. Anll. Bot. Fennica 3: 358-367.

TÜXEN, R. 1979. Sigmeten und Geosigmeten, ihre Ordnung und ihre Bedeutung für Wissenschaft, Naturschutz und Planung. Biogeographica 16: 79-92.

TÜXEN, R. \& E. PREISSING. 1942. Grundbegriffe und Methoden zum Studium der Wasser-und Sumpfpflanzengesellschaften. Deutsche Wasser Wirtschaft 37: 10-17, 57-69. Stuttgart.

VALLEJOS, L. 1991. Las formaciones vegetales del NE del Dpto. Curuzú Cuatia (Corrientes) y su relación con los suelos. Parodiana 6: 323-335.

VALLEJOS, L. 1993. La vegetación herbácea y suelos de un área del NW de Corrientes Argentina. Parodiana 8: 219-326.

VARELA, M.E., M.A. CORALES, G. TELL, A. POI DE NEIFF \& J.J. NEIFF. 1978. Estudios limnológicos en la cuenca del Riachuelo (Corrientes, Argentina).V. Biota acuática de los "embalsados" de la Laguna La Brava y caracteres del habitat. Ecosur 5: 97-118.
VERETTONI, H. 1961. Las asociaciones halófilas del partido de Bahía Blanca. Tesis doctoral. Facultad de Ciencias Naturales y Museo, Universidad Nacional de La Plata.

VERETTONI, H. 1965. Contribución al conocimiento de la vegetación psamófila de Bahía Blanca. Bahía Blanca.

VERETTONI, H. 1951a. Resultados de un viaje a la cuenca de la Laguna Verde (Tinogasta, Catamarca). III. Observaciones sobre la vegetación entre Tinogasta y la cuenca. Act. XV Sem. Geogr. Mendoza.

VERVOORST, F. 1951b. Zur Vegetation am Salar de Pipinaco in der Provinz Catamarca. Die Erde 1950/51 (2): 190-191.

VERVOORST, F. 1967. La vegetación de la República Argentina. VII: Las comunidades vegetales de la Depresión del Salado (provincia de Buenos Aires). Serie Fitogeográfica 7. INTA.

VILLAGRA, P. \& F.A. ROIG. 1999. Vegetación de las márgenes del río Mendoza (Mendoza Argentina) en su zona de divagación. Kurtziana 27: 309-317.

VILLAGRÁN, C. 1980. Vegetationsgeschichtliche und Pflanzensoziologische Untersuchungen im Vicente Pérez Rosales National Parck. Diss. Bot. 54.

VISCHI, N., A. OGGERO, A.L. CORREA \& S. SUÁREZ. 1999. Comunidades vegetales del bosque serrano y su asociación con factores edáficos. Bol. Soc. Argent. Bot. 34: 10-12.

VON HUMBOLDT, A. 1845. Kosmos: A General Survey of the Physical Phenomena of the Universe. Vol I. London: Hyppolyte Bailliere, Publisher.

VON ROCHOW, M. 1951. Die Pflanzengesellschaften des Kaiserstuhls. Fischer, Jena.

WALTER, H. 1967a. Das Pampaproblem in vergleichend okologischer Betrachtung und seine Losung. Erdkunde 21: 181-203.

WALTER, H. 1967b. The pampa problem and its solution. 2nd Intern. Semin. on Integrated Surveys of Nat. Grazing Areas, Delft. Publ. ITC-UNESCO Centre for Integr. Surv.

WARD, R.T. \& M.J. DIMITRI. 1966. Alpine tundra on Mt. Catedral in the Southern Andes. New Zealand J. Bot. 4: 42-56.

WEBER, H.E., J. MORAVEC \& J.P. THEURILLAT. 2000. International code of phytosociological nomenclature. $3^{\text {rd }}$ edition. J. Veg. Sci. 11: 739-768.

WERNER, D. 1972. Campo Arenal (NW-Argentinien), eine landschaftsokologische Detailstudie. Biogeographica. I. Okologie der Biosphare 75-86. Den Haag.

WERNER, D. 1974. Landschaftsokologische Untersuchungen in der argentinischen Puna. Tagungsber. u. wiss. Abh. Deutsch. Geographentag Kassel 1973: 508-528. Wiesbaden 
WERNER, D. 1978. Höhenstufen als Gesellschaftcomplexe. Ihre pflanzenzosiologische Abgrezung und Kartierung am Ostrande der argentinischen Puna. In: Tüxen, R (Hrsg.), Assozitionskomplexe (Sigmeten). Ber. Sympos. Internat. Vereinig. F. Vegetationsk., Rinteln 1977: 223-239. Vaduz.

WERTH, E. 1906-1908. Die Vegetation der subantarktischen Inseln, Kerguelens Possession und Heard Island. Is II. Dtsch. Südpolarexped. 1901-02, Bot. 8.

WESTHOFF, V., J.W. DIJK \& H. PASSCHIER. 1946. Overzicht der plantengemeenschappen in Nederland (Overview of the plant communities in the Netherlands). B.W. Breuchel, Amsterdam.

WESTHOFF, V. \& E. VAN DER MAAREL. 1978. The Braun-Blanquet approach. In: Whittaker, R.H. (ed.), Classification of plant communities: 287-399. Dr. W. Junk Publ., The Hague.

WILL, H. 1887. Die Vegetationsverhältnisse des Exkursionsgebietes der Deutschen Polarstation auf Süd-Georgien. Bot. Centralb1. 29: 256-257, 281283.

WILL, H. 1890. Die Vegetationsverhältnisse SüdGeorgiens. Ergebn. dtsch. Polarexped. Allg. Teil, 2: 172-194.

YU, S. \& L. ORLÓCI. 1990. On niche overlap and its measurement. Community Ecol. (Coenoses) 5: 159165.

ZABALZA, M.I., J.C. BARREIX \& E. CANO. 1989. Relevamiento fitosociológico del Parque Nacional Lihuel Calel, La Pampa, Argentina. Revista Fac. Agron., UN La Pampa 4: 69-94.

ZAIXSO, H.E. \& C.T. PASTOR. 1977. Observaciones sobre la ecología de los Mitilidos de la Ría Deseado. 1. Distribución y análisis biocenótico. Ecosur 4: $1-46$.

Recibido 16 de Junio de 2015, aceptado 25 de Abril de 2016.

\begin{abstract}
ANEXO
Relevamientos florísticos típicos (Relevé Typus) ver versión "On line".

A manera de ejemplo se agregan 195 relevamientos florísticos designados como Holotypus cuando se definió la asociación vegetal; en aquellos casos en que no se lo estableció originalmente se definió a uno como Lectotypus. En cada caso se mantuvo el epíteto específico del momento de la publicación. Los valores de cobertura específica corresponden a la escala mencionada en la metodología fitosociológica. Cada relevamiento está indicado con un número en la lista sintaxonómica a nivel de Asociación.
\end{abstract}




\section{E. Martínez Carretero et al. - Prodromus Sinsistemático y Estudios Fitosociológicos}

\section{ÍNDICE}

I. Alnetea acuminatae

II. Ambrosietea elatioris

III. Anthochloo lepidulae-Dielsiochloetea floribundae

IV. Artemisietea vulgaris

V. Asteretea vahlii

VI. Baccharidetea grisebachii

VII. Calamagrostietea vicunarum

VIII. Cakiletea maritimae

IX. Carico-Calthetea

X. Cedrelo fissilis-Ocoteetea puberulae

XI. Chiliotrichetea diffusi

XII. Deschampsio-Asteretea

XIII. Distichlio humilis-Anthobryetea triandri

XIV. Dolychlasietea lagascae

$\mathrm{XV}$. Elyonuretea mutici

XVI. Empetro-Bolacetea gummiferae

XVII. Empetro-Pernettyetea

XVIII. Festucetea gracillimae

XIX. Festucetea thermari

XX. Hippuretea vulgaris

XXI. Honkenyo-Elymetea

XXII. Hordeetea lechleri

XXIII. Hordeetea pubiflori

XXIV. Hordeetea santacrucensis

XXV. Lantano camarae-Chusqueetea ramosissimae

XXVI. Larreetea divaricato-cuneifoliae

XXVII. Lemnetea

XXVIII. Molinio-Arrhenatherea

XXIX. Mulino-Junellietea scopariae

XXX. Myrteolo-Sphagnetea

XXXI. Nothofagetea pumilionis-antarcticae

XXXII. Notholaenetea nivea

XXXIII. Panicetea racemosi

XXXIV. Panico urvilleani-Sporoboletea rigentis

XXXV. Pellaeetea tenuifoliae

XXXVI. Phragmito-Magnocaricetea

XXXVII. Piptochaetio-Stipetea neesianae

XXXVIII. Pistio stratiotes-Eichhornietea crassipedis

XXXIX. Plantagini rigidae-Distichietea muscoidis

XL. Polypodio squamulosi-Tillandsietea usneoidis

XLI. Podocarpo parlatorei-Tipuanetea tipi

XLII. Quinchamalio-Pernettyetea pumilae
XLIII. Rostkovietea

XLIV. Ruderali-Manihotetea utilissima

XLV. Salicetea humboldtianae

XLVI. Salici humboldtiani-Prosopietea albae

XLVII. Salvinio minimae-Lemnetea valdivianae

XLVIII. Sarcocornietea perennis

XLIX. Senecionetea bipontini

L. Senecioni-Proustietea cuneifoliae

LI. Soncho-Bidentetea pilosi

LII. Stellarietea mediae

LIII. Stipo-Lycietea fusci

LIV. Suaedetea divaricatae

LV. Verrucarietea

LVI. Wintero-Nothofagetea

LVII. Xyrido carolinianae-Typhetea domingensis

LVIII. Comunidades de epífitos del NE

Argentina (sintaxones de orden superior)

\section{Sintaxones probables en Argentina}

LIX. Acacio feddeannae-Prosopidetea ferocis

LX. Bidentetea tripartitae

LXI. Cladietea jamaicencis

LXII. Deuterocohnio longipetalae-Puyetea ferrugineae

LXIII. Eichhornietea crassipedis

LXIV. Fabiano bryoidis-Stipetea frigidae

LXV. Galio aparines-Urticietea dioicae

LXVI. Lemnetea minoris

LXVII. Lippio bolivianae-Dodonaetea viscosae

LXVIII. Loxopterygio grisebachii-Schinopsietea haenkeanae

LXIX. Nicotiano glutinosae-Ambrosietea arborescentis

LXX. Opuntietea sphaericae

LXXI. Polylepidetea tarapacano-besseri

LXXII. Polygono-Poetea annuae

LXXIII. Ruprechtio triflorae-Aspidospermetea quebracho-blanconis

\section{Sintaxones sin rango}

Se citan 1368 unidades de vegetación descriptas para Argentina sin asignarles pertenencia a alguna clase sintaxonómica. 
Bol. Soc. Argent. Bot. 51 (3) 2016

\section{Anexo}

\section{Ambrosietea elatioris}

Rel. 1

Alternanthera philoxeroides

Rumex obovatus

Sonchus oleraceus

Gamochaeta pensylvanica

Cyperus rotundus

Apium leptophyllum

Cynodon dactylon

Polygonum punctatum

Parietaria debilis

Acicarpha tribuloides

Rumex crispus

Paspalum urvilliei

Scutellaria racemosa

Setaria geniculata

Cuphea racemosa

Vicia epetiolaris

Ruellia tweediana

Dichondra microcalyx

Sporobolus indicus

Gomphrena celosioides

Rel. 2

Parietaria debilis

Coronopus didymus

Amaranthus viridis

Euphorbia prostrata
$+$

$+$

$+$

$+$
Gamochaeta pensylvanica

Conyza bonariensis var. angustifolia +

Conyza albida

Ambrosia elatior

Eleusine indica

Commelina erecta

Ipomnoea sp.

Cyperus sp.

\section{Rel. 3}

Cynodon dactylon

Eleusine tristachya

Gomphrena celosioides

Paspalum notatum

Hypochaeris chillensis

Medicago polymorpha

1

Soliva pterosperma

Plantago myosuros

\section{Rel. 4}

Pteris vittata

Pilea microphylla

Barbula indica

Didymodom australasiae

Pohlia papillosa

Tortula muralis

Tortella humulis

Parietaria debilis 
E. Martínez Carretero et al. - Prodromus Sinsistemático y Estudios Fitosociológicos

Euphorbia prostrata

Stemodia verticillata

Stachys gilliesii

Rumex obtusifolius

Scutellaria racemosa

\section{Asteretea vahlii}

Rel. 5

Arenaria serpens

Azorella trifoliolata

Rumex crispus

Cardamine valdiviana

Eleocharis pachycarpa

Chenopodium sp.

Nothoscordum bonariense

Carex barrosii

Rel. 6

Eleocharis pachycarpa

Senecio zozterifolius

Azorella trifoliolata

Hydrocotyle chamaemorus

Aster vahli

Juncus stipulatus

Carex decidua

Eleocharis macrostachys

Agrostis magellanica

Rel. 7

Littorella australis $+\quad$ Ranunculus hydrophilus $\quad 2$

$+\quad$ Fonckia uliginosa 2

$+\quad$ Eleocharis maculosa 2

$+\quad$ Crassula peduncularis 1

+ Eleocharis pachycarpa 1

Isolepis inundata 2

Isolepis cernua + +

Lilaeopsis macloviana $\quad+$

Sisyrinchium patagonicum $\quad+$

2

$+\quad$ Rel. 8

+ $\quad$ Limosella lineata 3

$1 \quad$ Crassula peduncularis 2

$+\quad$ Cardamine valdiviana $\quad 1$

$\begin{array}{lll}+ & \text { Plagiobotrys corymbosa } & 2\end{array}$

$+\quad$ Downingia pusilla 1

Lilaeopsis macloviana $\quad 1$

Rel. 9

2 Isoetes savatieri

$1 \quad$ Downingia pusilla 2

3 Limosella lineata 1

$+\quad$ Eleocharis pachycarpa $\quad 1$

2

2 Rel. 10

$1 \quad$ Colobanthus quitensis 1

$1 \quad$ Crassula moschata 2

Plantago barbata 5

Lilaeopsis macloviana $\quad 2$

Leptinella scariosa $\quad 2$ 
Bol. Soc. Argent. Bot. 51 (3) 2016

Deschampsia laxa

Isolepis cernua

Agrostis magellanica

Aster vahlii

\section{Rel. 11}

Senecio smithii

Marsippospermum grandiflorum

Hierochloe redolens

Poa robusta

Juncus lesuerii

Porothamnium arbusculans

Pratia repens

Galium antarcticum

Racomitrium lanuginosum

Carex darwinii v. urolepis

Apium australe

Leptinella scariosa

Ranunculus biternatus

Isolepis cernua

Aster vahlii

Festuca monticola

Asplenium dareoides

\section{Baccharidetea grisebachii}

Rel. 12

Senecio smithii

Poa robusta

Carex darwinii v. urolepis

Apium australe

\section{Rel. 13}

Baccharis grisebachii

Artemisa mendozana v. paramilloensis 1

Junellia asparagoides

Bougainvillea spinosa

\section{Rel. 14}

Discaria trinervis 5

Berberis empetrifolia 3

Satureja parviflora $\quad 1$

Baccharis petiolata +

Polypogon monspeliensis $\quad 1$

\section{Calamagrostietea vicunarum}

\section{Rel. 15}

Jarava chrysophylla var. chrysophylla 4

Jarava humilis

Lycium fuscum

Acantholippia deserticola

Tetraglochin cristatum

\section{Carico-Calthetea}

\section{Rel. 16}

Caltha sagittata

Carez gayana var. densa 3

Colobanthus quitensis 4

Plantago barbabata +

Deyeuxia poaeoides +

Carex decidua + 
E. Martínez Carretero et al. - Prodromus Sinsistemático y Estudios Fitosociológicos

Stellaria stenopetala

Ricardia gergensis

Euphrasia antarctica

Poa pratensis

Taraxacum officinale

Acaena magellanica

Rel. 17

Cortaderia pilosa var. minima

Chiliotrichium rosmarinifolium

Festuca scabriuscula

Schoenus andinus

Caltha sagittata

Valeriana macrorhiza

Pernettya pumila

Carex gayana

\section{Rel. 18}

Juncus scheuchzerioides

Caltha sagittata

Gentianella magellanica

Carex gayana

Juncus lesuerii

Musgo

Prataia repens

Trifolium repens

Rumex acetocella

Rel. 19

Juncus scheuchzerioides $+\quad$ Gunnera magellanica 5

$+\quad$ Caltha sagittata $\quad 1$

$+\quad$ Epilobium cioliatum 1

$+\quad$ Schoenus andinus $\quad 1$

$+\quad$ Valeriana macrorhiza 1

+ Patosia clandestina 1

Carex chillanensis $\quad 1$

Euphrasia subexserta $\quad 1$

Carex gayana var. gayana $\quad 1$

Phleum alpinum +

Musgo 1

Calceolaria palenae $\quad 1$

\section{Chiliotrichetea diffusi}

Rel. 20

Elymus antarcticus

Senecio mustersii

Chiliotrichum diffusum 3

Acaena pinnatifida

Sisyrinchium patagonicum +

+ Osmorhiza chilensis +

$+\quad$ Ribes magellanicum +

$+\quad$ Ranunculus peduncularis $\quad+$

$+\quad$ Baccharis magellanica +

$+\quad$ Festuca gracillima +

+ Perezia recurvata +

$+\quad$ Azorella trifurcata +

Carex macloviana +

Trifolium repens 2

Holcus lanatus 3 
Bol. Soc. Argent. Bot. 51 (3) 2016

Taraxacum officinale

Myosotis stricta

Hypochaeris radicata

Phleum alpinum

Deschampsia flexuosa

Festuca magellanica

Trifolium dubium

\section{Rel. 21}

Anemone multifida

Chiliotrichum diffusum

Discaria chacaye

Calceolaria biflora

Osmorhiza chilensis

Galium aparine

Bromus setifolius

Baccharis magellanica

Festuca gracillima

Perezia recurvata

Silene magellanica

Azorella trifurcata

Taraxacum officinale

Empetrum rubrum

Deschampsia flexuosa

Aacena magellanica

Phaiophleps biflora

\section{Rel. 22}

Elymus antarcticus

Osmorhiza chilensis
$+$

$+$

$+$

$+$

3

$+$

$+$

2

$+$

$+$

$+$

$+$

$+$

$+$

$+$

$+$

$+$

$+$
Chiliotrichum diffusum

Acaena pinnatifida

Lathyrus magellanicus

Ribes magellanicum

Blechnum penna-marina

2

Geum magellanicum

Galium aparine

Carex maclovina

Poa pratensis

Trifolium repens

Taraxcum officinale

2

Phleum alpinum

Deschampsia flexuosa

2

Codonoschis lessonii

Azorella lycopodioides

Lycopodium magellanicum

Rel. 23

Adesmia boronioides

4

Senecio laseguei

2

Valeriana carnosa

Bromus setifolius

Acaena sericea

2

Acaena splendens

1

Phacelia magellanica

Stipa brevipes

1

Mulinum spinosum

2

Agropyron patagonicum

Poa ibari

Viola maculata 
E. Martínez Carretero et al. - Prodromus Sinsistemático y Estudios Fitosociológicos

Festuca gracillima

Adesmia lotoides

Azorella caespitosa

Armeria maritima

Azorella fuegiana

Relbunium richardianum

Arjona patagonica

Acaena pinnatifida

Luzula alopecurus

Berberis empetrifolia

Taraxacum officinale

Perezia recurvata

Cerastium arvense

Rel. 24

Mulinum spinosum

Poa superbiens

Stipa brevipes

Anarthrophyllum desideratum

Gamocaheta affinis

Rytidosperma virescens

Discaria cacaye

Geranium patagonicum

Sisyrinchium patagonicum

Calceolaria biflora

Senecio patagonicus

Osmorhiza chilensis

Galium aparine

Bromus setifolius

Cotula scariosa
$+$

$+$

$+$

$+$

$+$

$+$

$+$

$+$

1

$+$

$+$

$+$

$+$

3

$+$

$+$

$+$

$+$

$+$

$+$

$+$

$+$

$+$

$+$

$+$

$+$

$+$

$+$
Baccharis magellanica

Viola maculata

Leucheria purpurea

Silene magellanica

Calceolaria biflora

Carex patagonica

Armeria maritima

Azorella trifurcata

Poa pratensis

Trifolium repens

Taraxacum officinale

Hypochaeris radicata

Berberis empetrifolia

Phleum alpinum

Deschampsia flexuosa

Thlaspi magellanicum

Adesmia lotoides

Saxifraga magellanica

Rel. 25

Vulpia myuros

Gamochaeta affinis

Acaena sericea

2

Vulpia megalura

Chiliotrichum diffusum

Discaria chacaye

Sisyrinchium patagonicum

Senecio patagonicus

3

Galium fuegianum

Bromus setifolius 
Bol. Soc. Argent. Bot. 51 (3) 2016

Baccharis magellanica

Perezia recurvata

Relbunium richardianum

Acaena platyacantha

Carex andicola

Erigeron myosotis

Azorella fuegiana

Armeria maritima

Poa pratensis

Myosotis stricta

Hypochaeris radicata

Deschampsia flexuosa

Acaena magellanica

Phaiophleps biflora

\section{Deschampsio-Asteretea}

Rel. 26

Deschampsia laxa

Lilaeopsis hillii

Plantago barbata

Colobanthus quitensis

Armeria maritima

Festuca purpurascens

Apium australe

Cardamine glacialis

Aster vahlii

\section{Rel. 27}

Alopecurus magellanicus

Carex darwinii
$+$

$+$

$+$

$+$

$+$

$+$

$+$

$+$

$+$

$+$

$+$

$+$

$+$
Senecio smithii

Apium australe

Gunnera magellanica

2

Hierochlöe redolens

1

Marsippospermum grandiflorum

Rel. 28

Deschampsia kingii

4

Cotula scariosa

1

Scirpus cernuus

1

Crassula moschata

1

Apium australe

Abrotanella linearifolia

Rel. 29

Agropyron magellanicum

3

Madia sativa

Senecio vulgaris

Arenaria serpens

Senecio philippi

Rumex acetosella

Acaena magellanica

Holcus lanatus

\section{Rel. 30}

Deschampsia kingii

Ourisia ruelloides

Luzula racemosa

Hypochaeris arenaria

Gamochaeta nivalis 
E. Martínez Carretero et al. - Prodromus Sinsistemático y Estudios Fitosociológicos

Arenaria serpens

Senecio philippi

Rumex acetosella

Acaena magellanica

Aster vahlii

Pernettya mucronata

Baccharis patagonica

Baccharis nivalis

Marsippospermum grandiflorum

\section{Dolychlasietea lagascae}

\section{Rel. 31}

Dolichlasium lagascae

Lobivia formosa

Junellia asparagoides

Lycium chanar

\section{Rel. 32}

Mutisia linifolia

Dolychlasium lagascae

Lobivia formosa

Bougainvillea spinosa

\section{Elyonuretea mutici}

\section{Rel. 33}

Axonopus fissifolius

Vernonia lithospermifolia

Melananthus multiflorus

Zornia gemella

Pfaffia tuberosa
$+$

$+$

1

1

$+$

2

$+$

$+$

$+$

2

$+$

$+$

$+$
Staelia thymoides

Schwenckia hirta

Eupatorium macrocephalum

Chamaecrista flexuosa

Desmanthus virgatus

Mecardonia montevidensis

Scoparia grisebachii

Eragrostis neesii

Panicum tricholaenoides

Andropogon lateralis

Eryngium ebracteatum

Macroptilium lathyroides

Gamocaheta pensylvanica

Ophioglossum sp.

Eragrostis bahiensis

\section{Rel. 34}

Lucilia acutifolia

Eupatorium inulaefolium

Lupinus guaraniticus

Macrosiphonia undulata

Verbena thymoides

Clerodendron ekmanii

Baaccharis pseudotenuifolia

Borreria marticrovettiana

Vernonia loretensis

Richardia grandiflora

Salvia durifolia

Hieracium commersonii

Butia yatay 
Bol. Soc. Argent. Bot. 51 (3) 2016

Aristida jubata

Eryngium pristis

Paspalum stellatum

Cnidoscolus albomaculatus

Aeschynomene falcata

Rynchosia corylifolia

Schinus weinmaniaefolius

\section{Rel. 35}

Panicum grumosum

Cuphea melvilla

Polygonum acuminatum

Ludwigia peruviana

Mimosa pigra

Cissus palmata

Cissampelos pareira

Aniseia argentina

Mikania dusenii

Hibiscus cisplatinus

Senna pendula

Pfaffia glomerata

Thelypteris interrupta

Vernonia scorpioides

Rel. 36

Polygonum acuminatum

Panicum grumosum

Rel. 37

Ocimum neurophyllum
Galphimia brasiliensis

Lippia sclerophylla

Lippia conterrmina

Borreria verticillata

Cuphea linrioides

Vigna peduncularis

Glechon ciliata

Rhynchosia diversifolia

Mimosa paupera

Rhynchosia pungens

Duddleia misionum

Eupatorium missionum

2

Lippia tegulifera

Croton landltii

Bidens riedelii

Sisyrinchium vaginatum

Aeschynomene falcata

Sida urticifolia

Rhynchosia corylifolia

Axonopus suffultus

Vernonia cognata

Borreria fastigiata

Dorstenia brasiliensis

Eriosema tacuareboese

Hypericum connatum

Baccharis tridentata

Schizachyrium bimucronatum

Croton misionum

Galactia marginalis

Melochia pyramidata 
E. Martínez Carretero et al. - Prodromus Sinsistemático y Estudios Fitosociológicos

Bernardia sp.

Eupatorium ellipticum

Rel. 38

Butia yatay

Croton sp.

Mimosa obtusifolia

Vernonia glabrata

Salvia nervosa

Heteropogon leptocladus

Borreria laxa

Clitoria epetiolata

Arachis villosa

Stevia entreriensis

Gymnopogon biflorus

Baccharis pedersenii

Axonopus argentinus

Sebasatiania hispida

Paspalum nicorae

Aeschinomene histrix

Jathropa isabellii

Noticastrum acuminatum

Cnidoscolus loasoides

Calea clausseniana

Vernonia cognata

Pfaffia tuberosa

Euphorbia hassleriana

Ruellia geminiflora

Hexachlamis humilis

Angelonia integerrima
$+$

$+$

V

2

$+$

$+$

$+$

2

$+$

1

$+$

$+$

1

$+$

$+$

1

$+$

1

$+$

$+$

1

$+$

$+$

$+$

$+$

$+$

$+$
Bernardia multicaulis

Borreria brachystemonoides

Macrosiphonia petrea

Waltheria comunis

Sida flavescens

\section{Rel. 39}

Zornia multinervosa

Chaptalia piloselloides

Pfaffia gnaphalioides

Turnera grandiflora

Evolvulus glomeratus

Stylosanthes macrosoma

Paspalum nicorae

Sida ciliaris

Eryngium nudicaule

Chamaecrista rotundifolia

Fimbristylis castanea

Paspalum plicatum

Desmodium incanum

Pleurophora saccocarpa

Rhynchospora praecincta

Lippia asperrima

Elyonorus muticus

Cyperus cayennensis

Cyperus sesquiflorus

Evolvulus sericeus

Schyzachirium microstachyum

Paspalum notatum

Galactia marginalis
2

1

5

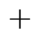

$+$

$+$

$+$

$+$$$
\text { . }
$$$$
+
$$$$
+
$$$$
1
$$$$
+
$$$$
2
$$$$
1
$$$$
+
$$$$
+
$$$$
+
$$$$
+
$$$$
1
$$$$
2
$$$$
+
$$$$
+
$$$$
+
$$$$
+
$$$$
5
$$$$
1
$$$$
1
$$$$
+
$$$$
+
$$$$
1
$$$$
1
$$ 
Bol. Soc. Argent. Bot. 51 (3) 2016

Andropogon selloanus +

Setaria geniculata

Sida spinosa

Panicum bergii

Commelina diffusa

Panicum hians

Eragrostis lugens

Spilanthes cdecumbens

Bulbostylis capillaris

Fimbristylis diphylla

Stachytarpheta cayennensis

Mecardonia montevidensis

\section{Empetro-Bolacetea gummifera}

Rel. 40

Calandrinia colchaguensis

Senecio kingii

Luzula alopecurus

Senecio lasaguei

Leucheria hahni

Empetrum rubrum

Bolax gummifera

Senecio magellanicus

Adesmia pumila

Trisetum spicatum ssp. cumingii

Festuca garcillima

Azorella caespitosa

Luzula chilensis

Deschampasia patula

Oxalis enneaphylla
Leucheria purpureae

Satureja darwinii

Plantago uniglumis

Draba magellanica

Thlaspi magellanicum

Phaiophleps biflora

Festuca magellanica

Perezia recurvata

Rumex acetosella

Nassauvia abbreviata

Festuca pyrogea

Deschampsia flexuosa

Silene magellanica

Senecio beaufilaii

Acaena poeppigiana

Rel. 41

Adesmia salicornioides

Luzula alopecurus

Empetrum rubrum

2

Bolax gummifera

Senecio magellanicus

Hypocheris incana

1

Erophila verna

Azorella fuegiana

Poa rigidifolia

2

Cerastium arvense

Nassauvia abbreviata

Festuca pyrogea

Taraxacum officinale 
E. Martínez Carretero et al. - Prodromus Sinsistemático y Estudios Fitosociológicos

Rel. 42

Lycopodium albofii

Carex caduca var. ortegae

Hieracium antarcticae

Berberis empetrifolia

Alopecurus magellanicus

Empetrum rubrum

Bolax gummifera

Senecio magellanicus

Azorella lycopodioides

Pernettya pumila

Saxifraga magellanica

Perezia pilifera

Chiliotricum diffusum

Draba magellanica

Acaena magellanica

Thlaspi magellanicum

Festuca magellanica

Nassauvia abbreviata

Festuca pyrogea

Calceolaria palenae

Nothofagus pumilio

Conostomum pentastichum

Rel. 43

Nassauvia juniperina

Leucheria hahnii

Nassauvia lagascae

Poa aff. rigidifolia
Hamadryas delfinii

Hamadryas kingii

Astragalus nivicola

Oreopolus glacialis

Nassauvia magellanica

Nassauvia revoluta

Senecio magellanicus

Azorella lycopodioides

Pernettya pumila

Saxifraga magellanica

Draba funiculosa

Cerastium arvense

Draba magellanica

Rumex acetosella

Nassauvia abbreviata

Festuca pyrogea

\section{Rel. 44}

Usnea trachycarpa

3

Perezia megalantha 1

Festuca aff. gracillima

Adesmia parviflora

Xerodraba pectinata

Onuris spegazzisiana

Senecio boelckei

Moschopsis rosulata

$+\quad$ Tristeum lasiolepis

$+\quad$ Hamadryas delfinii

$+\quad$ Agropyron fuegianum

$+\quad$ Hamadryas kingii
$+$

$+$

$+$

$+$

$+$

$+$

$+$

1

$+$

$+$

$+$

$+$

$+$

$+$

$+$

1 
Bol. Soc. Argent. Bot. 51 (3) 2016

Astragalus nivicola

Oreopolus glacialis

Nassauvia magellanica

Nassauvia revoluta

Nassauvia pygmaea

Bolax gummifera

Senecio magellanicus

Pernettya pumila

Pernettya pilifera

Azorella caespitosa

Calceolaria uniflora

Poa rigidifolia

Cerastium arvense

Festuca magellanica

Deschampsia flexuosa

Rel. 45

Onuris hatcheriana

Moschopsis sp.

Tristagma nivale

Oreopolus glacialis

Nassauvia revoluta

Valeriana magellanica

Astragalus nivicola

Perezia megalantha

Hamadryas kingii

Perezia pilifera

Senecio magellanicus

Saxifraga magellanica

Luzula alopecurus
Bolax gummifera

Empetrum rubrum

Leucheria hahnii

Colobanthus subulatus

Nassauvia darwinii

Oxalis enneaphylla

Vicia bijuga

Festuca pyrogea

Cerastium arvense

Rel. 46

Abrotanella emarginata

1

Lycopodium magellanicum +

Senecio boelckei

Empetrum rubrum

4

Bolax gummifera

2

Luzula alopecurus

Perezia pilifera

Senecio magellanicus

Pernettya pumila

Leucheria hahnii

Azorella lycopodioides

Festuca gracillima

Nassauvia darwinii

Poa poecila

Silene magellanica

Arjona patagonica

Gamochaeta nivalis

Cerastium arvense

Draba magellanica 
E. Martínez Carretero et al. - Prodromus Sinsistemático y Estudios Fitosociológicos

Deschampsia flexuosa

Acaena magellanica

Rumex acetosella

Nassauvia abbreviata

\section{Rel. 47}

Marsippospermum grandiflorum

Gunnera magellanica

Agropyron fuegianum

Acaena platyacantha

Alopecurus magellanicus

Carex atropicta

Blechnum penna-marina

Geum magellanicum

Chiliotricum diffusum

Berberis buxifolia

Phleum alpinum

Cerastium arvense

\section{Rel. 48}

Marsippospermum grandiflorum

Bolax gummifera

Pernettya pumila

Empetrum rubrum

Azorella lycopodioides

Agrostis flavidula

Taraxacum officinale

Juncus scheuchzerioides

Berberis buxifolia

Phleum alpinum
$+$

$+$

$+$

$+$

$+$

$+$

$+$

$+$

$+$

$+$

$+$

$+$
Festuca pyrogea

Festuca magellanica

\section{Rel. 49}

Festuca contracta

Trisetum phleoides

Abrotanella emarginata

3

Carex minutissima

Deyeuxia erytrostachya

Viola tridentata

2

Bolax gummifera

Azorella lycopodioides

Perezia pilifera

Oxalis enneaphylla

Draba magellanica

Thlaspi magellanicus

Deschampsia flexuosa

Cardamine glacialis

\section{Festucetea gracillimae}

\section{Rel. 50}

Euphorbia collina $v$. andina

Carex andina

Nardophyllum bryoides

Bromus setifolius

Polygala darwiniana

Festuca gracillima

3

Rytidosperma virescens

Colobanthus subulatus

Nassauvia darwinii
3

3

$+$

$+$

$+$

$+$

$+$

$+$ 
Bol. Soc. Argent. Bot. 51 (3) 2016

Hypochaeris incana

Azorella fuegiana

Hordeum comosum

Azorella caespitosa

Oxalis enneaphylla

Adesmia lotoides

Acaena platyacantha

Viola maculata

Acaena poeppigiana

Trisetum cumingii

Satureja darwinii

Cerastium arvense

Nassauvia abbreviata

Berberis buxifolia

\section{Rel. 51}

Agropyrum patagonicum

Adesmia villosa

Senecio miser

Vicia bijuga

Carex andina

Verbena ameghinoi

Festuca gracillima

Rytidosperma virescens

Nassauvia darwinii

Erophila verna

Deschampasia patula

Relbunium richardianum

Viola maculata

Acaena poeppigiana
Trisetum cumingii

Poa rigidifolia

Armeria maritima

Calceolaria uniflora

Luzula chilensis

Cerastium arvense

Senecio magellanicus

Deschampasia flexuosa

Trisetum lechleri

Nassauvia abbreviata

\section{Rel. 52}

Verbena prachardii

2

Philippiella patagonica +

Senecio culcitenellus

Burkartia lanigera

Carex andina

Nardophyllum bryoides

Bromus setifolius

Ephedra frustillata

Stipa ibari

Festuca gracillima

Rytidosperma virescens 3

Colobanthus subulatus 1

Nassauvia darwinii

Hypochaeris incana

Deschampsia patula

Relbunium richardianum

Poa rigidifolia

3

Armeria maritima 


\section{E. Martínez Carretero et al. - Prodromus Sinsistemático y Estudios Fitosociológicos}

Calceolaria uniflora

Satureja darwinii

Silene magellanica

Luzula chilensis

Perezia recurvata

Senecio magellanicus

Rel. 53

Verbena aff. chubutensis

Stipa ameghinoi

Nassauvia ulicina

Stipa ibari

Stipa psilantha

Verbena silvestrii

Verbena ameghinoi

Carex subandina

Plantago tehuelche

Ephedra frustillata

Colobanthus subulatus

Erophila verna

Poa dusenii

Azorella caespitosa

Acaena platyacantha

Mulinum spinosum

Cerastium arvense

Festuca magellanica

\section{Rel. 54}

Verbena tridens

Carex patagonica

$+$

$+$

$+$

$+$

$+$

$+$

Rel. 55

Valeriana moyanoi

Anarthrophyllum desideratum

$+$

$+$

$+$

$+$

$+$

$+$

$+$

$+$

2

$+$

$+$

$+$

$+$

2

$+$

$+$

$+$

$+$

$+$

Stipa ibari

Verbena silvestrii

Bromus catharticus

Taraxacum officinale

Verbena silvestrii

Alstroemeria patagonica

Benthamiella patagonica

Euphorbia collina

Poa dusenii

Azorella caespitosa

Oxalis laciniata

Acaena platyacantha

Mulinum spinosum

Cersatium arvense

Hamadryas delfinii

Nassauvia abbreviata

\section{Rel. 56}

Gutierrezia baccharioides

Stipa ameghinoi

Stipa ibari

Nardophyllum bryoides

Verbena ameghinoi

Bromus setifolius

Mulinum valentinii

Festuca garcillima
2

1

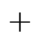

$+$

4

$+$

$+$

$+$

$+$

$+$

$+$

$+$

$+$

$+$

1

$+$

1

1

$+$

$+$

2 
Bol. Soc. Argent. Bot. 51 (3) 2016

Adesmia lotoides

Nassauvia darwinii

Poa dusenii

Calceolaria uniflora

Silene magellanica

Satureja darwinii

Armeria maritima

Hypochaeris incana

Festuca magellanica

Pahiophleps biflora

Perezia recurvata

\section{Rel. 57}

Adesmia boronioides

Amsinckia calycina

Polemonium micranthum

Capsella bursa-pastoris

Lathyrus magellanicus

Descurainea antarctica

Bromus setifolius

Erodium cicutarium

Relbunium richardianum

Viola maculata

Berberis buxifolia

Myosotis stricta

Taraxacum officinale

Cerastium arvense

\section{Rel. 58}

Geranium sessiliflorum
Festuca gracillima

Colobanthus subulatus

Poa rigidifolia

Viola maculata

Armeria maritima

Oxalis enneaphylla

Azorella fuegiana

Rytidosperma virescens

Relbunium richardianum

Luzula chilensis

Oxalis laciniata

Empetrum rubrum

Trisetum cummingii

Azorella trifurcata

2

Hordeum pubiflorum

Poa pratensis

Taraxacum officinale

2

Phleum alpinum

Acaena magellanica

Rumex acetosella

Deschampsia flexuosa

Phaiophleps biflora

Draba magellanica

Cerastium arvense

Festuca magellanica

\section{Rel. 59}

Oreomyrrhis hoockeri

Festuca arundinacea

Sagina procumbens 
E. Martínez Carretero et al. - Prodromus Sinsistemático y Estudios Fitosociológicos

Myosotis discolor

Erigeron myosotis

Uncinia macrolepis

Festuca gracillima

Poa ibari

Chiliotrichum diffusum

Calceolaria palenae

Cotula scariosa

Azorella trifurcata

Hordeum pubiflorum

Dactylis glomerata

Poa pratensis

Trisetum lechleri

Trifolium repens

Cerastium fontanum

Geranium patagonicum

Phleum alpinum

Acaena magellanica

Rumex acetosella

Deschampsia flexuosa

Berberis buxifolia

Thlaspi magellanicum

Draba magellanica

Festuca magellanica

Taraxacum gilliesii

Colobanthus quitensis

Rel. 60

Oreomyrrhis hoockeri

Madia sativa
$+$

$+$

$+$

2

$+$

$+$

$+$

$+$

$+$

2

2

$+$

$+$

$+$

$+$

$+$

$+$

2

2

1

$+$

$+$

$+$

$+$

$+$

$+$

$+$

$+$

$+$
Erigeron myosotis

Festuca gracillima

3

Colobanthus subulatus

Hypochaeris incana

Rytidosperma virescens

Baccharis magellanica

Cotula scariosa

Senecio kingii

Azorella trifurcata

Gentianella magellanica

Poa pratensis

1

Trifolium repens

Cerastium fontanum

Phleum alpinum

Acaena magellanica

Rumex acetosella

2

Deschampsia flexuosa

Thlaspi magellanicum

Phaiophleps biflora

Festuca magellanica

Taraxacum gilliesii

Rel. 61

Senecio martinensis

Festuca gracillima

Viola maculata

2

Calceolaria uniflora

2

Satureja darwinii

Chiliotrichum diffusum

Calceolaria palenae 
Bol. Soc. Argent. Bot. 51 (3) 2016

Blechnum penna-marina

Juncus scheuchzerioides

Cardamine glacialis

Pratia repens

Empetrum rubrum

Eriachaenium magellanicum

Bolax gummifera

Azorella lycopodioides

Trisetum lechleri

Rel. 64

Hypochaeris radicata

Artemisia magellanica

Phleum alpinum

Puccinellia parviflora

Rumex acetosella

Hordeum lechleri

Deschampsia flexuosa

Rumex crispissimus

Berberis buxifolia

Rel. 65

Thlaspi magellanicum

Hydrocotyle chamaemorus

Draba magellanica

Potentilla anserina

Cerastium arvense

Taraxacum gilliesii

Luzula alopecurus

Perezia pilifera

Galium magellanicum

Ranunculus hygrophilous

Hordeum lechleri

Deschampsia antartica

Alopecurus magellanicus

\section{Festucetea thermari}

Carex macloviana

Rel. 62

Agrostis tenuis

Hippuris vulgaris

Trifolium repens

Alopecurus pratensis

$+$

Deyeuxia poaeoides

Carex gayana

Carex decidua

Carex gayana

$+$

Acaena magellanica

\section{Hordeetea lechleri}

\section{Cotula scariosa}

\section{Hordeetea pubiflori}

Rel. 66

Juncus balticus

Plantago maritima

Rel. 63

Poa atropidiformis

Hordeum lechleri

Scorzonera laciniata 
E. Martínez Carretero et al. - Prodromus Sinsistemático y Estudios Fitosociológicos

Festuca pallescens

Azorella fuegiana

Acaena poeppigiana

Sisyrinchium junceum

Nassauvia darwinii

\section{Rel. 67}

Euphrasia antarctica

Geranium sp.

Festuca pallescens

Carex sorianoi

Hordeum pubiflorum

Azorella trifurcata

Carex macloviana

Alopecurus magellanicus

Koeleria fuegiana

Juncus scheuchzerioides

Aster vahlii

Vicia magellanica

Galium fuegianum

Acaena pinnatifida

Colobanthus subulatus

Gamocaheta nivalis

Senecio magellanicus

Pernettya pumila

Berberis buxifolia

Carex subantarctica

Myosotis stricta

Erigeron myosotis
3

1

$+$

$+$

$+$

$+$

$+$

4

$+$

1

$+$

$+$

$+$

$+$

$+$

$+$

$+$

$+$

$+$

$+$

$+$

$+$

$+\quad$ Rel. 70

$+\quad$ Limosella australis

$+\quad$ Nostoc sp.

$+\quad$ Ranunculus pseudotrullifolius

$+\quad$ Pratia repens

Eleocharis albibracteata
3

$+$

2

$+$

1

1

$+$

$+$

$+$

$+$

$+$

$+$

5

$+$

$+$

$+$

4

3

$+$

4

4 
Bol. Soc. Argent. Bot. 51 (3) 2016

Deschampsia antarctica

\section{Rel. 71}

Carex macrosolen

Pratia repens

Hordeum pubiflorum

Euphrasia antarctica

Carex vallis-pulchrae

Alopecurus magellanicus

Arjona pusilla

Eleocharis albibracteata

Colobanthus quitensis

Juncus scheuchzerioides

Poa anua

Cerastium fontanum

\section{Rel. 72}

Rumex maritimus

Pratia repens

Hordeum pubiflorum

Euphrasia antarctica

Alopecurus magellanicus

Carex gayana

Caltha sagittata

Deschampsia antarctica

Poa annua

Veronica serpyllifolia

Hordeetea santacrucensis

Rel. 73

$$
\begin{array}{ll}
\text { Plagiobothrys calandrinioides } & + \\
\text { Hordeum santacrucense } & 1
\end{array}
$$

Poa atropidiformis var. patagoinica + Azorella caespitosa

Colobanthus subulatus

\section{Rel. 74}

Scutellaria nummulariaefolia 3

Hordeum santacrucense

Colobanthus subulatus

\section{Rel. 75}

Poa atropidiformis

2

Alopecurus aequalis

Acaena platyacantha

Hordeum santacrucense

Bromus caharticus

\section{Rel. 76}

Alopecurus aequalis

Juncus balticus

Eriachenium magellanicum 5

Pratia repens 1

Plagiobothrys calandrinioides +

Myosurus patagonicus

Poa atropidiformis var. patagonica +

Plantago barbata +

Acaena poeppigiana 2

Adesmia lotioides +

Colobanthus subulatus + 
E. Martínez Carretero et al. - Prodromus Sinsistemático y Estudios Fitosociológicos

Hordeum lechleri

Cerastium arvense

Adesmia villosa

\section{Rel. 77}

Puccinellia biflora

Hordeum halophyllum

Suaeda patagonica

Polygonum aviculare

Chenopodium carnosolum

Boopis australis

Heliotropium patagonicum

Hordeum santacrucense

Scorzonera laciniata

Hordeum lechleri

Juncus scheuchzerioides

Festuca magellanica

Rel. 78

Scirpus spegazzianus

Scirpus nevadensis

Suaeda patagonica

Scorzonera laciniata

Puccinellia mendozina

\section{Rel. 79}

juncus balticus

Puccinellia magellanica

Boopis australis

Hordeum santacrucense
$+$

$+$

$+$

$+$

$+$

$+$

$+$

$+$

$+$

$+$
Taraxacum officinale

Festuca pyrogea

Mulinum spinosum

Lepidium pseudo-didynus

\section{Larreetea divaricato-cuneifoliae}

Rel. 80

Larrea divaricata

3

Fabiana denudata 2

Condalia microphylla 1

Bouteloua curtipendula

Stipa tenuis

Eragrostis lugens

Trichocereus candicans

Thymophylla belenidium

Acantholippia seriphioides

Opuntia sulphurea

Senna aphylla

Sporobolus cryptandrus

Gochnatia glutinosa

Schinus fasciculata

4

Stipa ichu

3

Artemisia mendozana

2

Trycicla spinosa

2

Lycium chilense

Diplache dubia

\section{Rel. 81}

Larrea cuneifolia

4

Junellia aspera 
Bol. Soc. Argent. Bot. 51 (3) 2016

Atriplex lampa

Pappophorum philippianum

Lycium chilense

Opuntia sulphurea

Trichocereus candicans

Lycium tenuispinosum

Acantholippia seriphioides

\section{Rel. 82}

Chuquiraga rosulata

Stipa hypsophila

Lesquerella mendocina

Evolvulus sericeus

Bouteloua curtipendula

Opuntia sulphurea

Senna aphylla

Acantholippia seriphioides

Prosopis flexuosa var. depressa

Prosopidastrum globosum fma.

Gutierrezia spathulata

Ephedra ochreata

Aristida mendocina

\section{Rel. 83}

Lycium vergarae

Puna clavarioides

Pterocactus meglioli

Larrea divaricata

Junellia aspera

Scleropogon brevifolius
$+$

$+$

$+$

$+$

1

$+$

$+$

\section{Rel. 84}

3

$+$

$+$

$+$

2

$+$

2

$+$

$+$

$+$

$+$

$+$

$+$

$+$

$+$

$+$

$+$

Rel. 85
Larrea nitida

Lycium chanar

Elymus erianthus

Stipa vaginata

Fabiana denudata

Stipa chrysophylla var. crispula

Adesmia trijuga

Aphyllocladus sanmartinianus

Sisymbrium arnottianum

Larrea divaricata

Glandularia crithmifolia

Dipyrena glaberrima

Salvia gilliesii

Lobivia formosa

Molinio-Arrhenatherea

Trisetum lechleri

3

Poa pratensis 2

Holcus lanatus +

Trifolium repens 4

Dactylis glomerata +

Hypochaeris radicata 2

Baccharis magellanica 2

Armeria maritima 1

Festuca gracillima 1

Viola maculata

Rumex acetosella 
E. Martínez Carretero et al. - Prodromus Sinsistemático y Estudios Fitosociológicos

Berberis buxifolia

Myosotis stricta

Rel. 86

Leucanthemum vulgare

Hordeum tetraploideum

Poa pratensis

Cerastium fontanum

Holcus lanatus

Trifolium repens

Plantago lanceolata

Achillea millefolium

Agrostis tenuis

Vulpia bromoides

Taraxacum officinale

Nothofagus antarctica

Viola maculata

Berberis buxifolia

\section{Rel. 87}

Cirsium vulgare

Trifolium hybridum

Sonchus oleraceus

Poa pratensis

Cerastium fontanum

Holcus lanatus

Vulpia bromoides

Geranium patagonicum

Nothofagus antarctica

Acaena ovalifolia
$+$

$+$

$+$

$+$

$+$

\section{$\begin{array}{r}\text { Rel. } 89 \\ \hline\end{array}$}

1

\section{Rel. 88}

Blechnum penna-marina

Ribes magellanicum

Baccharis patagonica

Acaena pinnatifida

Armeria maritima

Festuca gracillima

Berberis buxifolia

Pernettya mucronata

Discaria chacaye

Embothrium coccineum

Osmorhiza chilensis

\section{Mulino-Junellietea scopariae}

Colliguaja intgerrima

4

Baccharis pulchella

2

Baccharis trimera

Junellia scoparia

Eupatorium buniifolium

Schinus fasciculata

Artemisia mendozana

Berberis grevilleana

Cerastium arvense

Poa ligularis

Dypirena glaberrima

Calceolaria brunellifolia

Lycium chilense

Stipa tenuissima 
Bol. Soc. Argent. Bot. 51 (3) 2016

Adesmia aff. campestris

Oenothera aff. magellanica

Junellia juniperina

Erodium cicutarium

Poa resinulosa

Cerastium arvense

Adesmia horrida

Taraxacum officinale

Bromus brevis

Nothoscordum sp.

Ephedra breana

Stipa speciosa

Gamochaeta stachydifolia

Lesquerella mendocina

\section{Rel. 90}

Nassauvia axillaris

Adesmia colluteioides

Festuca rubra var.

Adesmia uspallatensis

Calceolaria brunellifolia

Perezia carduncelloides

Mulinum spinosum

Tetraglochin alatum

Cerastium arvense

Poa ligularis

Maihueniopsis ovata

\section{Rel. 91}

Adesmia uspallatensis
Junellia asparagoides

Elytrigia mendocina

Calceolaria brunellifolia

Perezia cardunceloides

Cerastium arvense

1

Bowlesia tropaeolifolia

Poa ligularis

Stipa paramilloensis

Rel. 92

Artemisia echegarayii

3

Senecio uspallatensis

Solanum juncalense

Baccharis thymifolia

Mulinum spinosum

Tetraglochin alatum

2

Cerastium arvense

Erodium cicutarium

Junellia scoparia

Bowlesia tropaeolifolia

Poa resinulosa

Junellia juniperina

Stipa paramilloensis

Verbascum thapsus

3

Lycium chilense

1

\section{Rel. 93}

Adesmia horrida

Nassauvia axillaris

Elytrigia scabriglumis 
E. Martínez Carretero et al. - Prodromus Sinsistemático y Estudios Fitosociológicos

Pachylaena atriplicifolia

Cajophora coronata

Solanum juncalense

Sisyrhinchium macrocarpum

Festuca desvauxi

Oxalis sp.

\section{Rel. 94}

Fabiana peckii

Stipa speciosa var. atuelensis

Festuca acanthophylla

Grisebachiella hieronymi

Ephedra andina

Schinus roigii

Grindelia chiloensis

Ephedra ochreata

Stipa speciosa var. major

Rhodophyala mendocina

Baccharis darwinii

Anarthrophyllum elegans

Brachyclados lycioides

Panicum urvilleanum

Budleja mendocensis

Stipa scirpea

Acantholippia seriphioides

Senna aphylla

Prosopis flexuosa var. depressa

Adesmia campestris

Rel. 95
$+$

$+$

$+$

$+$

$+$

$+$

$+$

$+$

$+$

$+$

$+$

$+$

1

$+$

1

2

$+$

$+$

$+$

$+$

$+$

$+$

$+$

$+$

Retanilla patagonica

Adesmia retrofracta

Adesmia renjifoana

Grindelia chilonsis

Gutierrezia spathulata

Junellia seriphioides

Fabiana peckii

Panicum urvilleanum

Rel. 96

Neosparton aphyllum

Junellia alatocarpa

Nicotiana spegazzinii

Grindelia chiloensis

Junellia seriphioides

Baccharis darwinii

Panicum urvilleanum

Poa lanuginosa

Lycium chilense

Bougainvillea spinosa

Rel. 97
Colliguaja integerrima

2

Maihueniopsis glomerata

2

Bredemeyera microphylla

Prosopis flexuosa var. depressa

2

Chuquiraga erinacea ssp. hystrix +

2

Junellia connatibracteata

Chenopodium papulosum

3

$+$ 
Bol. Soc. Argent. Bot. 51 (3) 2016

Hordeum comosum

Bromus tectorum

Gutierrezia solbrigii

Loasa bergii

Gilia patagonica

Sisyrinchium macrocarpum ssp.

Adesmia corymbosa

Tropaeolum incisum

Ephedra ochreata

Junellia ligustrina

Stipa neaei

Mulinum spinosum

Tetraglochin alatum

Senecio filaginoides

Bromus setifolius

Boopis anthemoides

Mutisia retrorsa

Nassauvia axillaris

\section{Rel. 98}

Fabiana patagonica

Stillingia patagonica

Neosparton aphyllum

Chuquiraga erinaceae ssp. hystrix

Schinus roigii

Senna arnottiana

Orobanche chilensis

Maihueniopsis glomerata

Grindelia chiloensis

Stipa neaei
Maihuenia patagonica

Stipa speciosa v. media 2

Anarthrophyllum rigidum $\quad 1$

Rhodophyala mendocina +

Astragalus pehuenches

Stipa speciosa var. manclequensis

Poa lanuginosa

\section{Rel. 99}

Adesmia pinifolia

3

Phacelia secunda

Jaborosa kurtzii

Malesherbia lirana var. subglabrifolia +

Melosperma andicola

Mulinum spinosum

Bromus setifolius

Acaena sp.

Poa holciformis

\section{Rel. 100}

Stipa chrysophylla var. crispula 3

Elymus erianthus

Stipa tenuis

Muhlenbergia torreyi 2

Fabiana peckii

Stipa chrysophylla var. chrysophylla + Junellia seriphioides

Stipa vaginata

Bredemeyera microphylla

$+$

Larrea nitida 
E. Martínez Carretero et al. - Prodromus Sinsistemático y Estudios Fitosociológicos

Stipa tenuissima

1

Rel. 101

Stipa chrysophylla var. crispula

1

Stipa speciosa var. manclequensis 2

Senecio bracteolatus var. bracteolatus 1

Stipa speciosa var. media

Grindelia chiloensis

Stipa chrysophylla

Ephedra ochreata

Gutierrezia spathulata

Junellia seriphioides

Senecio subulatus

Rhodophyala mendocina

Schinus fasciculta

Panicum urvilleanum

Poa lanuginosa

Bougainvillea spinosa

Lycium chilense

Rel. 102

Stipa speciosa var. media

Gallardoa fisheri

Brachyclados megalanthus

Condalia megacarpa

Stipa chrysophylla var. crispula

Elymus erianthus

Chuquiraga erinacea ssp. erinacea

Poa durifolia

Brachyclados lycioides

2

$+$

$+$

$+$
Boopis gracilis

Stipa humilis

1

Grindelia chiloensis

1

Gutierrezia spathulata

1

Junellia seriphioides

1

Junellia alatocarpa

Larrea nitida

Mutisia retrorsa

Plantago patagonica

Hyalis argentea

Euphorbia collina

Prosopis ruiz-lealii

Rel. 103

Poa durifolia

3

Junellia asparagoides

Ephedra breana

Gutierrezia spathulata

Glandularia flava

Schinus roigii

Calceolaria brunellifolia

Mulinum spinosum

Tetraglochin alatum

Anarthrophyllum elegans

Arjona longifolia

\section{Myrteolo-Sphagnetea}

Rel. 104

Schizaea fistulosa

Gunnera lobata 
Bol. Soc. Argent. Bot. 51 (3) 2016

Marsippospermum grandiflorum 1

Oreobolus obtusangulus

Acaena pumila

Abrotanella linearifolia

Schoenus antarcticus

Astelia pumila

Donatia fascicularis

Drosera uniflora

Gaimardia australis

Caltha dioneifolia

Lebetanthus myrsinites

Nothofagus antarctica

\section{Rel. 105}

Juncus stipulatus

Oxalis echegarayi

Gunnera lobata

Marsippospermum grandiflorum

Myrteola nummularia

Caltha appendiculata

Perezia lactucoides

Cortaderia pilosa

Acaena pumila

Abrotanlla linearifolia

Schoenus antarcticus

Nothofagus antarctica

Empterum rubrum

Pernettya mucronata

Chiliotrichum diffusum

Pilgirodendrom uviferum
Luzuriaga marginata

Lebetanthus myrsinites

Escallonia serrata

\section{Rel. 106}

Sphagnum magellanicum

Carex magellanica

Gunnera lobata

Tetroncium magellanicum

Pernettya pumila

Caltha appendiculata

Nanodea muscosa

Oreobolus opbtusangulus

Schoenus antarcticus

Astelia pumila

Donatia fascicularis

Empetrum rubrum

Racomitrium willii

2

Nothofagus betuloides

2

Pilgirodendron uviferum

1

Tribeles australis

Racomitrium lanugisonum

Rel. 107

Carex microglochin

Carex curta

Sphagnum magellanicum

3

Carex magellanica

Pernettya pumila

Empetrum rubrum 
E. Martínez Carretero et al. - Prodromus Sinsistemático y Estudios Fitosociológicos

Pernettya mucronata

Nothofagus betuloides

Luzuriaga marginata

Uncinia tenuis

Juncus balticus

Carex darwinii

\section{Rel. 108}

Cladonia glacialis

Sphagnum magellanicum

Gunnera lobata

Tetroncium magellanicum

Marsippospermum grandiflorum

Myrteola nummularia

Pernettya pumila

Caltha appendiculata

Nanodea muscosa

Oreobolus obtusangulus

Acaena pumila

Carpha alpina

Empetrum rubrum

Pernettya mucronata

Blechnum penna-marina

Pilgirodendron uviferum

Gunnera magellanica

\section{Rel. 109}

Cryptochila grandiflora

Senecio trifurcatus

Perezia magellanica
Lycopodium confertum

Bolax caespitosa

4

Phyllachne uliginosa 2

Drapetes muscosus

Ortechene rariflora

Oreobolus obtusangulus

Pernettya pumila

1

Gaimardia australis

2

Thamnolia vermicularis

Tapeinia pumila

Caltha dionaefolia

Schoenus antarcticus

Carpha alpina

Embothrium coccineum

Rel. 110

Cryptochila grandiflora

5

Dacrydium fonckii

Gaimardia australis

Thamnolia versicularis

Tapeinia pumila

Caltha dionaefolia

Myrteola nummularia

2

$+$

Acaena pumila

Drosera uniflora

Pernettya mucronata

\section{Rel. 111}

Azorella selago

Lycopodioum alboffii 
Bol. Soc. Argent. Bot. 51 (3) 2016

Bolax caespitosa 3

Phyllachne uliginosa

Drapetes muscosus

Ortechne rariflora

Pernettya pumila

Caltha dionaefolia

Embothrium coccineum

Rel. 112

Bolax caespitosa

Phyllachne uliginosa

Oreobolus obtusangulus

Pernettya pumila

Caltha dionaefolia

Carpha alpina

Cortaderia pilosa

Nanodea muscosa

Astelia pumila

Baccharis patagonica

Rostkovia magellanica

\section{Rel. 113}

Conostomum pentastichum

Cloraea gaudichaudii

Perezia megellanica

Lycopodium confertum

Oreobolus obtusangulus

Pernettya pumila

Dacrydium fonckii

Gaimardia australis
Donatia fascicularis

Thamnolia versicularis

Tapeinia pumila

Caltha dionaefolia

Caltha appendiculata

Myrteola nummulariua

Schoenus antarcticus

Acaena pumila

Drosera uniflora

Perezia lactucoides

Astelia pumila

Nothofagus betuloides

2

Baccharis ilicifolia

Escallonia serrata

Pernettya mucronata

\section{Nothofagetea pumilionis-} antarcticae

\section{Rel. 114}

Araucaria araucana

Nothofagus pumilio

Anemone antucensis

Festuca scabriuscula

Gavilea lutea

Valeriana laxiflora

Perezia prenanthoides 1

Poa tristigmatica 1

Brachytecium sp +

Adenocaulon chilense 2

Myoschilos oblongum +

Codonorchis lessonii 1 
E. Martínez Carretero et al. - Prodromus Sinsistemático y Estudios Fitosociológicos

Blechnum penna-marina

Arachnitis uniflora

Osmorhiza chilensis

Barbula depressa

\section{Rel. 115}

Nothofagus pumilio

Pernettya myrtilloides var. nana

Rubus geoides

Maytenus disticha

Ribes cucullatum

Empetrum rubrum

\section{Rel. 116}

Trisetum caudulatum

Antenaria chilensis

Nothofagus antarctica

Agropyron fugianum

Hieracium antarcticum

Poa nemoralis

Poa patagonica

Osmorhiza chilensis

Galium aparine

Ribes magellanicum

Blechnum penna-marina

Cotula scariosa

Chiliotrichum diffusum

Viola maculata

Taraxacum officinale

Thlaspi magellanicum

+
+
1
+

Rumex acetosella

Festuca pyrogea

Cerastium arvense

Taraxacum giliesii

Nassauvia abbreviata

Phleum alpinum

\section{Rel. 117}

Festuca purpurscens

Nothofagus antarctica

Poa patagonica

Agoseris coronopifolia

Cryptopteris fragilis

Osmorhiza chilensis

Calceolaria palenae

Cardamine glacialis

Geum magellanicum

Vicia magellanica

Galium fuegianum

Acaena pinnatifida

Luzula alopecurus

Bromus catharticus

Poa pratensis

Holcus lanatus

Thlaspi magellanicum

Rumex acetosaella

Cerastium arvense

Draba magellanica

Acaena magellanica
2

$+$

4

$+$

$+$

$+$

$+$

$+$

$+$

$+$

$+$

$+$

$+$

$+$

$+$

$+$

$+$

$+$

$+$

$+$

$+$ 
Bol. Soc. Argent. Bot. 51 (3) 2016

Rel. 118

Nothofagus antarctica

Escallonia virgata

Bromus coloratus

Osmorhiza chilensis

Calceolaria palenae

Ribes magellanicum

Geum magellanicum

Vicia magellanica

Acaena ovalifolia

Galium fuegianum

Chiliotrichum diffusum

Acaena pinnatifida

Baccharis patagonica

Discaria chacaye

Azorella fuegiana

Taraxacum officinale

Holcus lanatus

Deschampsia flexuosa

Rumex acetosella

Berberis buxifolia

Rel. 119

Nothofagus antarctica

Gunnera magellanica

Macrachaenium gracilis

Adenocaulon chilense

Cerastium fontanum

Valeriana laphatifolia

Cryptopteris fragilis
Osmorhiza chilensis

Galium aparine

Ribes magellanicum

Codonorchis lessonii

Vicia magellanica

Blechnum penna-marina

Acaena ovalifolia

Cotula sacriosa

Pernettya mucronata

Viola maculata

Poa pratensis

Holcus lanatus

Cerastium fontanum

Thlaspi magellanicum

Berberis buxifolia

Taraxacum gilliesii

Blechnum magellanicum

Empetrum rubrum

Berberis ilicifolia

Rel. 120

Leymus arenarius

2

Polygala salasiana

Arenaria serpens

Viola maculata

$+\quad$ Acaena pinnatifida

+ Phacelia secunda

+ Cirsium vulgare

$+\quad$ Myosotis stricta

$+$

$+$

4

1

$+$

$+$

$+$

$+$

$+$

$+$

$+$

$+$

$+$

$+$

$+$

$+$

$+$

$+$

$+$

$+$

$+$

$+$

$+$

Taraxacum officinale 
E. Martínez Carretero et al. - Prodromus Sinsistemático y Estudios Fitosociológicos

Sagina procumbens

Rumex acetosella

Rel. 121

Senecio bracteolatus

Viola maculata

Acaena pinnatifida

Berberis buxifolia

Baccharis patagonica

Adesmia pumila

\section{Rel. 122}

Bromus setifolius

Agrostis imberbis

Araucaria araucana

Mulinum spinosum

Stipa speciosa

Bromus tectorum

Festuca scabriuscula

Poa tristigmatica

Senecio linarifolius

Juncus lesuerii

\section{Panico urvilleani-}

\section{Sporoboletea rigentis}

\section{Rel. 123}

Stipa scirpea

Sporobolus rigens

Grindelia chiloensis

Stipa speciosa var. media

Senecio filaginoides

\section{Rel. 124}

Hyalis argentea var. latisquama 3

Aristida inversa 2

Nicotiana petunioides $\quad+$

Doniophyton anomalum

Portulaca grandiflora

Echinopsis leucantha

Aristida adsencionis

1

Philibertia gilliesii

Panicum urvilleanum

Junellia seriphioides

Senecio subulatus

Verbena flava

Fabiana peckii

Trichocline cineraria

Verbecina encelioides

Prosopis flexuosa var. depressa

Bougainvillea spinosa

Lecanophora heterophylla

\section{Rel. 125}

Baccharis genistifolia

Sporobolus rigens $\quad V$

Senecio filaginoides $\quad V$

Panicum urvilleanum V

Baccharis divaricata III

Hyalis argentea var. latisquama III

Glycyrrhiza astragalina III

Poa lanuginosa V 
Bol. Soc. Argent. Bot. 51 (3) 2016

Adesmia filipes

Aster haplopappus

Rel. 126

Psila spartioides

Sporobolus rigens

Glycyrrhiza astragalina

Panicum urvilleanum

Prosopis strombulifera

Stipa speciosa

\section{Phragmito-Magnocaricetea}

Rel.127

Psila spartioides

Tessaria absinthioides

Distichlis spicata

Cardaria draba

Hypochaeris chondrilloides

Piptochaetio-Stipetea neesianae

Rel. 128

Stipa charruana

Carex bonariensis

Dichondra microcalyx

Stenotaphrum secundatum

Juncus imbricatus

Bromus mollis

Piptochaetium bicolor

Paspalum dilatatum

Eclipta bellidioides
III

III

Verbena montevidensis

Cypella hebertii

Alophia laua

1

Piptochaetium stipoides

2

Phyla canescens

V

V

IV

IV

IV

IV

Nothoscordum bonariensis

Eryngium echinatum

Ambrosia tenuifolia

Medicago hispida

Spilanthes decumbens

Lolium multiflorum

Gamochaeta spicata

Melilotus indicus

3

Apium leptophyllum

Cynara cardunculus

Torilis nodosa

Asclepis mellodora

Cirsium vulgare

Glandularia dissecta

Gaudinia fragilis

2

Bromus unilioides
Centaurea calcitrapa

Carduus acanthoides

Panicum berghii

\section{Rostkovietea}

\section{Rel. 129}

Rostkovia magellanica

Dicranoloma robustum 2

Eleocharis melanphala 
E. Martínez Carretero et al. - Prodromus Sinsistemático y Estudios Fitosociológicos

Eleocharis melanostachys

Drepanocladus $s p$.

Marsippospermum grandiflorum

Gunnera magellanica

\section{Salicetea humboldtianae}

\section{Rel. 130}

Salix humboldtiana

Tessaria integrifolia

Mikania periplocifolia

Solanum amygdalifolium

Funastrum clausum

\section{Rel. 131}

Cathormiom polyanthum

Ocotea suaveolens

Banara arguta

Ruprechtia brachysepala

Bergeronia sericea

Pithecellobium cauliflorum

Celtis spinosa

Psidium persicifolium

Eugenia moraviana

Psychotria carthagenensis

Cratevia tapia

Coccoloba morongii

Nectandra microcarpa

Scleria schulzii

Rel. 132
2

$+\quad$ Solanum amygdalifolium

$+\quad$ Mikania periplocifolia

Cissus sicyoides

Cayaponia podantha

\section{Salvinio minimae-}

\section{Lemnetea valdivianae}

Rel. 133

5

Wolffiella lingulata

Limnobium laevigatum

Salvinia minima

Azolla caroliniana

5

Spirodela intermedia

Lemna valdiviana

2

Lemna minuta

1

Ricciocarpus natans 1

Wolffia columbiana 1

Wolffia brasiliensis

2

\section{Rel. 134}

Pistia striatiotes

5

Limnobium laevigatum 2

Utricularia gibba 2

Habenaria aranifera +

Enhydra anagalis $\quad 1$

Scirpus cubensis 1

Eichhornia crassipes +

Wolffiella lingulata 1

Azolla caroliniana 1

Lemna minuta 
Bol. Soc. Argent. Bot. 51 (3) 2016

Rel. 135

Panicum grumosum

Cuphea melvilla

Polygonum acuminatum

Ludwigia peruviana

Mimosa pigra

Cissus palmata

Cissampelos pareira

Anisela argentina

Mikania dusenii

Hibiscus cisplatinus

Senna pendula

\section{Rel. 136}

Axonopus compressus

Sisyrinchium af. alatum

Vernonia glabrata

Cyperus reflexus

Aeschynomene montevidensis

Junellia ephedroides

Rhynchospora corymbosa

Cissampelos pareira

Macroptilium lathyroides

Commelina diffusa

Corchorus argutus

Panicum hians

Limnobium laevigatum

Salvinia minima

Azolla caroliniana
Spirodela intermedia

Lemna valdiviana

Lemna minuta

1

Ricciocarpus natans 1

Wolffia columbiana

1

Wolffia brasiliensis

\section{Sarcocornietea perennis}

Rel. 137

2

Sarcocornia ambigua

Puccinellia biflora

$v$

$+$

Rel. 138

Salicornia ambigua

Atriplex aff. macrostyla

Puccinellia glaucescens

Suaeda argentinensis

4

2

2

2

2

Rel. 139

2

Limonium brasiliense

Sarcocornia perennis

Distichlis spicata

1

Frankenia junniperioides 3

Prosopis strombulifera

Rel. 140

Nithrophila australis var. kuntzei 3

Distchlis scoparia 2

Puccinella glaucescens 1

Ranunculus cymbalaria 
E. Martínez Carretero et al. - Prodromus Sinsistemático y Estudios Fitosociológicos

Triglochin concinna

Distichlis spicata

Hypochaeris chondrilloides

Juncus lesueurii

Taraxacum officinale

Bromus tectorum

Grindelia chiloensis

Hymenolobus procumbens

Descurainia argentina

Rumex crispus

Centaurea calcitrapa

Lepidium perfoliatum

\section{Rel. 141}

Sarcocornia ambigua

Atriplex macrostylis

Senecio miser

Lepidophyllum cupressiforme

Rel. 142

Puccinellia mendocina

Poa ibari

Lepidophyllum cupressiforme

Poa poecilla

Colobanthus subulathus

Stipa ibari

Viola maculata

Perezia recurvata

Poa patagonica
$+$

$+$

$+$

$+$

$+$

$+$

$+$

$+$

$+$

$+$

$+$

$+$

$+$

4

$+$

4

$+$

2

$+$

4

$+$

$+$

$+$

$+$

$+$

$+$
Rel. 143

Frankenia chubutensis

Senecio miser

1

Lepidophyllum cupressiforme 4

Hordeum comosum

Stipa chrysophylla

Nassauvia ulicina

Hordeum pubiflorum

\section{Stellarietea mediae}

Rel. 144

Solanum tuberosum

Allium sativum

Daucus carota

Diplachne uninervia var. procumbens +

Echinochloa cruss-galli

Sonchus oleraceus

Amaranthus quitensis

Eragrostis virescens

Anoda cristata

1

Sorghum halepense

Paspalum distichum

Pitraea cuneato-ovata

Chenopodium album

Convolvulus arvensis

\section{Rel. 145}

Baccharis pingraea

Kochia scoparia

Melilotus albus 
Bol. Soc. Argent. Bot. 51 (3) 2016

Bromus catharticus +

Bassia hyssopifolia

Rel. 146

Allium cepa

Echinochlöa cruss-galli

Sonchus oleraceus

Sisymbrium irio

Amaranthus quitensis

Eragrostis virescens

Euphorbia serpens

Cynodon dactylon

Pitraea cuneato-ovata

Chenopodium album

Convolvulus arvensis

Galinsoga parviflora

Cynara cardunculus

Plantago lanceolata

Bromus unioloides

Rel. 147

Zea mays

Setaria verticillata

Echinochlöa cruss-galli

Sonchus oleraeus

Amaranthus quitensis

Eragrostis virescens

Anoda cristata

Cynodon dactylon

Paspalum distichum

1

1

$+$

2

1

$+$

$+$

$+$

$+$

2

$+$
Pitraea cuneato-ovata

Chenopodium album

Convolvulus arvensis

Kochia scoparia

Galinsoga parviflora

Foeniculum vulgare

\section{Rel. 148}

Lycopersicum esculentum

2

Portulaca oleracea

Echinochloa crus-galli

Sonchus oleraceus

Sisymbrium irio

Amaranthus quitensis

Eragrostis virescens

Anoda cristata

Flaveria bidentis

Pitraea cunato-ovata

2

\section{Rel. 149}

Solanum tuberosum

4

Allium sativum

Portulaca oleracea

1

Datura ferox

$+\quad$ Echinochlöa cruss-galli

$+\quad$ Sisymbrium irio

1

$+\quad$ Amarathus quitensis +

$+\quad$ Anoda cristata

$+\quad$ Flaveria bidentis

Pitraea cuneato-ovata 
E. Martínez Carretero et al. - Prodromus Sinsistemático y Estudios Fitosociológicos

Chenopodium album

Rel. 150

Bromus catharticus

Foeniculum vulgare

Solidago chilensis

Tessaria absinthioides

Distichlis spicata

Hypochaeris chondrilloides

Cynara cardunculus

Chenopodium album

Pitraea cuneato-ovata

Rubia tinctorum

Phragmites comunis

Phyla nodiflora

\section{Rel. 151}

Imperata condensata

Equisetum giganteum

Muehlenbergia asperifolia

Hypochaeris chondrilloides

Foeniculum vulgare

Solidago chilensis

Cynodon dactylon

Baccharis salicifolia

Baccharis pingraea

Melilotus alba

\section{Rel. 152}

Vitis vinifera

1

Wedelia glauca

Cynodon dactylon

Baccharis salicifolioa

Baccharis pingraea

Cichorium intybus

Pitraea cuneato-ovata

Convolvulus arvensis

Bromus unioloides

Foeniculum vulgare

Sphaeralcea miniata

Pappophorum caespitosum

Trichloris crinita

Senecio subulatus

Diplachne dubia

\section{Rel. 153}

Solanum tuberosum 1

5

$+$

$+$

$+$

2

$+$

1

$+$

$+$

$+$

$+$

$+$


Bol. Soc. Argent. Bot. 51 (3) 2016

Junelia juniperina

Bromus brevis

Sphaeralcea mendocina

Stipa hypsophila

\section{Rel. 154}

Cynodon dactylon

Baccharis salicifolia

Conyza bonariensis

Plantago major

Hydrocotyle bonariense

Wedelia glauca

Melilotus albus

\section{Rel. 155}

Allium cepa

Lepidium draba

Hoffmannseggia glauca

Pitraea cuneato-ovata

Convolvulus arvensis

Kochia scoparia

\section{Rel. 156}

Solanum tuberosum

Hordeum distichum

Lepidium bonariensis

Plantago patagonica

Veronica peregrina ssp xalepensis

Carduus nutans var. macrocephalus

Cirsium vulgare
$+$

$+$

$+$

$+$

$+$

$+$

$+$

$+$

$+$

5

$+$

$+$

$+$

1
Taraxacum officinale

Polygonum aviculare

Plantago lanceolata

Vicia pampicola

Oxalis sp.

Erigeron $s p$.

Oenothera picensis

Grindelia chiloensis

Stipa tenuissima

Cerastium arvense

Poa ligularis

Rel. 157

Solanum tuberosum

2

Hordeum distichum

Chenopodium album

5

Solanum atriplicifolium

Xanthium spinosum

Sisymbrium altissimum

Erodium cicutarium

Convolvulus arvensis

1

Chenopodium frigidum

Carduus nutans var. macrocephalus + Cynara cardunculus

Taraxacum officinale

1

Polygonum aviculare

Rumex crispus

1

Sphaeralcea mendocina

Rel. 158 


\section{E. Martínez Carretero et al. - Prodromus Sinsistemático y Estudios Fitosociológicos}

Wedelia glauca

Vicia fava

Echinochloa cruss-galli

Paspalum distichum

Solanum elaeagnifolium

Pitraea cuneato-ovata

Chenopodium album

\section{Rel. 159}

Diplotaxis erucoides

Portulaca oleracea

Sorghum halepense

Flaveria bidentis

Echinochloa crusgalli

Pitraea cuneato-ovata

Sonchus oleraceus

Chenopodium album

Euphorbia serpens

Rumex crispus

Datura ferox

Diplotaxis muralis

Cynodon dactylon

Bromus catharticus

\section{Stipo-Lycietea fusci}

\section{Rel. 160}

Artemisia paramilloensis

Bougainvilela spinosa

Chuquiraga erinacea ssp. hystrix

Lycium chanar
$+$

$+$

$+$

$+$

$+$

$+$

$+$
Adesmia echinus

Rel. 161

Bacharis incarum

1

Lycium chanar 1

Stipa scirpea var. parva $\quad 1$

Adesmia horrida

Senecio filaginoides

Elymus erianthus

Maihueniopsis glomerata

1

Fabiana denudata

Stipa paramilloensis

\section{Rel. 162}

Lycium fuscum

1

Chuquiraga erinacea ssp. hystrix +

Menonvillea cuneata

Junellia asparagoides

Mulinum spinosum

Adesmia horrida

2

Senecio uspallatensis

Artemisia mendozana var. paramilloensis 2

Junellia erinacea

Calandrinia picta

Gilia foetida

Elymus erianthus

Larrea nitida

2

Tricycla spinosa

Maihueniopsis glomerata 
Bol. Soc. Argent. Bot. 51 (3) 2016

\section{Rel. 163}

Jarava chrysophylla var. chrysophylla 1 Jarava vaginata

Viola volcanica

Hordeum pubiflorum ssp. halophyllum +

Euphorbia sp.

Jaborosa parviflora

Jaborosa caulescens var. bipinnatifida +

Jarava humilis

1

Cisthante picta

Baccharis incarum

Rel. 164

Jarava speciosa var. abscondita

Tetraglochin cristatum

Leymus erianthus

Rel. 165

Junellia asparagoides

Senecio grindeliaefolius

Gallardia tontalensis

Bougainvillea spinosa

Senecio uspallatensis

Stipa chrysophylla

Calandrinia picta

Gilia foetida

Gomphrena pumila

Phacelia sinuata

Elymus erianthus

Maihueniopsis glomerata

$+$

$+$

1

$+$

$+$
Stipa vaginata

Poa resinulosa

Rel. 166

Crypthanta albida

Polemonium micranthum

Jaborosa caulescens

1

Phacelia sinuata

1

Sphaeralcea philippiana

Galdularia parodii

Chenopodium frigidum

Calycera herbacea

Ipomopsis gossypifera

Elymus erianthus

Rel. 167

Ephedra breana

2

Lycium chilense

Tarassa antofagastana

Junellia minutiflora

Senecio filaginoides

3

Spaeralcea philippiana

Glandularia parodii

Chenopodium frigidum

Senecio uspallatensis

Cisthante picta

$+\quad$ Gilia foetida

Gomphrena pumila

Elymus erianthus

Maihueniopsis glomerata 
E. Martínez Carretero et al. - Prodromus Sinsistemático y Estudios Fitosociológicos

Rel. 168

Sencio filaginoides

Stipa scirpea

Stipa chrysophylla fma. modica

Lesquerella mendocina

Ephedra andina

Mulinum ulicinum

Senecio uspallatensis

Cisthante picta

Gilia foetida

Gomphrena pumila

Maihueniopsis glomerata

Fabiana denudata

\section{Rel. 169}

Senecio filaginoides

Phacelia sinuata

Stipa vaginata fma. rigida

Junellia uniflora

Trichocline cineraria

Astragalus arnottianus

Stipa speciosa fma. abscondita

Senecio oreinus

Senecio aff. gunckelii

Stipa speciosa var. parva

Ephedra andina

Adesmia horrida

Stipa chrysophylla

Stipa chrysophylla var. crispula
Gomphrena pumila

Elymus erianthus

Junellia aspera

3

$+$

$+$

$+$

$+$

$+$

$+$

$+$

$+$

$+$

1

$+$

$+$

3

$+$

$+$

$+$

$+$

$+$

$+\quad$ Rel. 171

$+\quad$ Plazia daphnoides

3

$+\quad$ Ephedra multiflora +

2

$+$

$+$

Rel. 170

Stipa plumosa 2

Digitaria californica

1

Bothryochloa springfieldii

Menodora decemfida

3

Erioneuron pilosum

Salvia gilliesii

Elymus erianthus

Lycium chanar

Stipa scirpea

Gilia foetida

Stipa paramilloensis

Bougainvillea spinosa

Larrea nitida

Junellia aspera

Thymophylla belenidium

Bredemeyera microphylla

Dolychlasium lagascae

1

Stipa hypsophila

Stipa humilis v. ruiziana

Melixa chilensis

Salvia gilliesii

$+$

$+$

$+$ $+$ $+$ 1 1 $+$ $+$ $+$ $+$ $+$ $+$ $+$ $+$ $+$ $+$ 
Bol. Soc. Argent. Bot. 51 (3) 2016

Elymus erianthus

$+$

Lycium chanar

Maihueniopsis glomerata

\section{Rel. 172}

Sisymbrium arnottianum

Poa resinulosa

Descurainia cummingiana

Poa ligularis

Stipa humilis var. ruiziana

\section{Rel. 173}

Gymnophyton polycephalum

Dolychlasium lagascae

Lobivia formosa

Junellia asparagoides

Lycium chanar

Bougainvillea spinosa

Adesmia horrida

\section{Rel. 174}

Halophytum ameghinoi

Atriplex rosea

\section{Wintero-Nothofagetea}

\section{Rel. 175}

Tepualia stipularis

Pseudopanax laetevirens

Grammitis magellanica

Pernettya mucronata
$+$

$+$

$+$

$+$

2

$+$

3

$+$

$+$

$+$

$+$

$+$

5

1

2

$+$

$+$

3
Hebe elliptica

Nothofagus betuloides

Hymenophyllum secundum

Lebetanthus myrsinites

Philesia magellanica

Schoenus antarcticus

\section{Rel. 176}

Sticta caulescens

Maytenus magellanica

Gunnera magellanica

Nothofagus betuliodes

2

Drimys winteri

Berberis ilicifolia

Lebetanthus myrsinites

2

Philesia magellanica

Grammitis magellanica

Blechnum magellanicum

Luzuriaga marginata

Desfontainia spinosa

3

Pseudopanax laetevirens

\section{Rel. 177}

Lomatia ferruginea

Macrachaenium fragile

Escallonia serrata

Embothrium coccineum

Berberis buxifolia

Blechnum penna-marina

Pernettya mucronata 
E. Martínez Carretero et al. - Prodromus Sinsistemático y Estudios Fitosociológicos

Maytenus magellanica

Nothofagus betuloides

Drimys winteri

Berberis ilicifolia

Senecio acanthifolious

Uncinia tenuis

Luzuriaga marginata

Pilgerodendron uviferum

Desfontainea spinosa

Dacrydium fonckii

\section{Rel. 178}

Empetrum rubrum

Baccharis nivalis

Racomitrium lanuginosum

Stereoculon aff. ramulosum

Andreasa wilsonii

Embothrium cocineum

Pernettya mucronata

Nothofagus antarctica

Nothofagus betuloides

\section{Rel. 179}

Pernettya mucronata

Gunnera magellanica

Acaena magellanica

Hierochloe redolens

Poa robusta

Festuca purpurascens

Marsippospermum grandiflorum

\section{Rel. 180}

Empetrun rubrum

Pernettya mucronata

Maytenus magellanica 1

Chiliotrichum diffusum 1

Gunnera magellanica 4

Acaena magellanica

Nothofagus betuloides

Berberis milicifolia

Holcus lanatus

Taraxacum officinalis

\section{Comunidades de epífitas del NE Argentino}

\section{Rel. 181}

Schlotheimia rugifolia

Leptodontium viticulosoides +

Macrocoma orthotrichoides 4

Frullania ericoides 2

Radula aurantii

Metzgeria myriopoda

Leptogium margineilum

2

Physcia sorediosa

\section{Rel. 182}

Vriesia fruburgensis var. tucumanensis 2

Tillandsia tenuifolia

Aechemea distichantha 2

Pleurotallis sp. 1

Helicodontium capillare $\quad 1$ 
Bol. Soc. Argent. Bot. 51 (3) 2016

Frullania platycalyx

Papillaria sp.

Rel. 183

Orthostichopsis tenuis

Squamidium brasiliense

Papillaria nigrescens

Zelometeorium ambiguum

Radula aurantii

Meteorium illecebrum

Cheilolejeunea clausa

Lejeunea cephalandra

Leptogium marginellum

Orthostichopsis $s p$.

\section{Rel. 184}

Neckeropsis undulata

Pilotrichella cyathipoma

Hildebrandtiella guyanensis

Pseudocryphaea domingensis

Metzgeria myriopoda

Porella brasiliensis

Plagiochila martiana

Racopilum tomentosum

Cheilolejeunea clausa

Lejeunea filipes

Aphanolejeunea clavatopapillata

Lejeunea laetevirens

Chryso-hypnum diminutivum

Lophocolea coadunata

\section{Rel. 185}

Hymenodon aeruginosus

Asplenium scandicinum

Campyloneuron phyllitidis

Polypodium succum

Radula macrostachya

Lejeunea flava

Lejeunea laetevirens

Lejeunea cancellata

Metzgeria myriopoda

Pilotrichella cyathipoma

Plagiochila raddiana

Dimerella sp.

Schiffneiolejeunea polycarpa

Lejeunea tapajosensis

Rhizomorphasp.

\section{Rel. 186}

Porotrichum lancifrons

$\begin{array}{ll}\text { Plagiochila raddiana } & 2\end{array}$

Aphanolejeunea sp.

Lejeunea saetiloba

Plagiomnium rhynchophorum

2

\section{Rel. 187}

Hypopterigium tamariscinum

4

Plagiomnium rhynchophorum

Racopilum tomentosum 
E. Martínez Carretero et al. - Prodromus Sinsistemático y Estudios Fitosociológicos

Taxileujenea obtusangula

Haploclacdium microphyllum

Helicodontium capillare

\section{Rel. 188}

Uleastrum paraguensis

Fabronia jamesonii

Hypotrachyna dactylifera

\section{Rel. 189}

Vesicularia vesicularis

Callicostella pallida

Isopterygium tenerum

Ciclodictyon varians

Leujeunea quinqueumbonata

Racopilum tomentosum

Lophocolea coadunata

Plagiomnium rhynchophorum

Rel. 190

Tillandsia duratii

Tillandsia meridionalis

Tillandsia loliacea

Tillandsia vernicosa

\section{Rel. 191}

Erpodium beccarii

Erpodium glaziovii

Tortula pagorum

Frullania ericoides
2

$+$

1

\section{Rel. 192}

Microgramma vaccinifolia 2

4

$+$

1

Tillandsia tricholepis

Pleurotallis recurva

Rhipsalis leucorhaphis

Tillandsia loliacea

Frullania ericoides

Radula aurantii

Metzgeria psilocraspeda

Forsstroemia producta

Sematophyllum subpinnatum

Polypodium squalidum

Leptogium marginellum

Phyllopsora parvifolia

Heterodermia albicans

Leptogium phyllocarpum

\section{Rel. 193}

Fissidens brevipes 3

Leptogium marginellum 3
Radula aurantii 3

Peperomia aceroana +

Phyllopsora parvifolia $\quad 1$

Rel. 194

Stereophyllum radiculosum 4

Frullania ericoides

3

3

3

1


Bol. Soc. Argent. Bot. 51 (3) 2016

Radula aurantii +

Letroutia dominguensis +

Rel. 195

Chryso-hypnum diminutivum 3

Isopterygium byssobolax 2

Isopterygium subbrevisetum +

Lejeunea setiloba $\quad 2$

Sematophyllum subsimplex +

Lejeunea quinqueumbonata + 

